\title{
DYNAMIC INVERSION AND BACKSTEPPING CONTROLLER ROBUSTNESS ANALYSIS FOR A REUSABLE LAUNCH VEHICLE
}

\author{
A Thesis \\ Presented to the Faculty of \\ California Polytechnic State University, \\ San Luis Obispo
}

\author{
In Partial Fulfillment \\ of the Requirements for the Degree \\ Master of Science in Aerospace Engineering
}

by

Janet Michi Coakley

February 2011 
(C) 2008

Janet Michi Coakley

ALL RIGHTS RESERVED 


\section{COMMITTEE MEMBERSHIP}

TITLE:

AUTHOR:

DATE SUBMITTED:
Dynamic Inversion and Backstepping Controller Robustness Analysis for a Reusable Launch Vehicle Janet Michi Coakley

February 2011 $\begin{array}{ll}\text { COMMITTEE CHAIR: } & \text { Eric Mehiel, PhD } \\ & \text { Cal Poly Aerospace Engineering Department Chair } \\ & \text { Cal Poly Aerospace Engineering Professor }\end{array}$

COMMITTEE MEMBER: Daniel Biezad, PhD

Cal Poly Aerospace Engineering Professor

COMMITTEE MEMBER: Jordi Puig-Suari Cal Poly Aerospace Engineering Professor

COMMITTEE MEMBER: Kurt Colvin, PhD

Cal Poly Industrial and Manufacturing Engineering Professor 


\author{
ABSTRACT \\ Dynamic Inversion and Backstepping Controller Robustness Analysis \\ for a Reusable Launch Vehicle \\ Janet Michi Coakley
}

The Air Force has been working towards developing technology for operationally responsive space (ORS), which is the ability to launch military assets into space without the long set up time currently required. Part of the solution to ORS is to develop a reusable booster vehicle capable of sending any vehicle into orbit, then descending back to the atmosphere and landing unpowered so that it may take another vehicle into orbit with a 48 hour turnaround time. Currently classical gain tuning techniques are used to design a controller for a specific mission, which may hinder the vehicle's ability to perform multiple missions if gains have to be re-tuned. Advanced nonlinear control methods like dynamic inversion and backstepping may eliminate the need to use classical gain tuning techniques that may increase quick turnaround time, reliability, and performance. Both methods consider the dynamics of the vehicle allowing the controller to be applied to the whole flight envelope. However, they are model-based methods that require knowledge of plant aerodynamics. The objective was to develop a backstepping outer loop and dynamic inversion inner loop controller for a reusable launch vehicle configuration and evaluate its robustness characteristics by inserting aerodynamic uncertainties into the static and control surface aerodynamic data separately and together. Both dynamic inversion and backstepping were susceptible to control surface aerodynamic uncertainties more than static aerodynamics. The benefit of using dynamic inversion and backstepping was that it was formulated so that it decouples the system of equations as long as the dynamics were modeled accurately. The control variable became a bank of decoupled integrators. However, when uncertainties were introduced into the plant model, the controller was unable to accurately model the dynamics, which reintroduced axes coupling inherent in the plant. The coupling caused performance in one axis to degrade if another axis degraded.

Keywords: Guidance, Control, Reusable launch vehicle, approach and landing, robustness, dynamic inversion. 


\section{ACKNOWLEDGMENTS}

First I would like to express my sincere gratitude to my advisor, Dr. Eric Mehiel, who adopted me as one of his grad students. This thesis would not have been possible without his continuous support to pursue my study. His knowledge and insightful feedback have helped shape my research and enabled me to complete this paper.

My sincere thanks also goes to Dan Salluce, my program lead at Northrop Grumman, for taking me under his wing and mentoring me. He provided me an opportunity to work on his projects during an internship that inspired me to pursue a career as a control engineer. He has also given me the idea for this thesis and provided the sanitized aerodynamic data used for my analysis.

I would like to thank the rest of my committee members, for their time and interest in my thesis subject. Their comments and questions allowed me to improve my analysis greatly.

I am also grateful to the Aerospace Engineering faculty, for providing a stimulating and fun environment to learn and grow as an engineer. Thank you for always being patient with me during office hours and taking the time to help me understand topics, homework, and project assignments.

I owe a great deal to family and friends for their unwavering encouragement, love, and support. You all knew the right things to say to keep me going at times when I was ready to break under pressure; but, also kept me in check when I was out of line. I am thankful for having such great people around me to continually inspire me to do better. 


\section{Table of Contents}

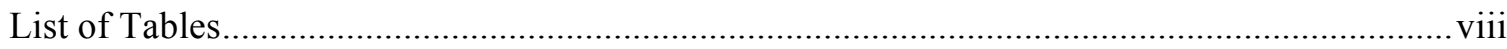

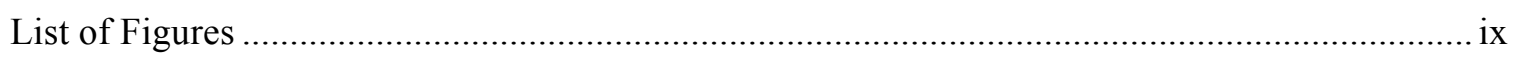

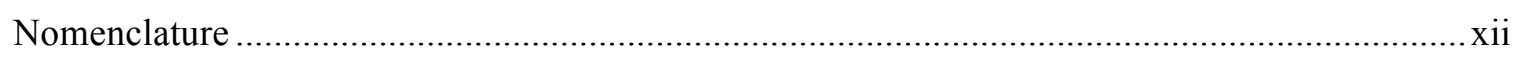

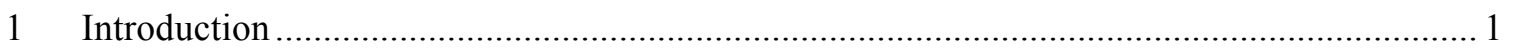

1.1 Background on Reusable Launch Vehicles............................................................ 1

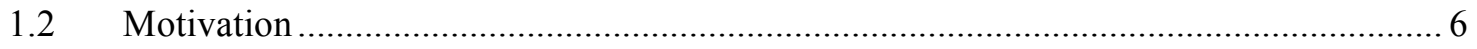

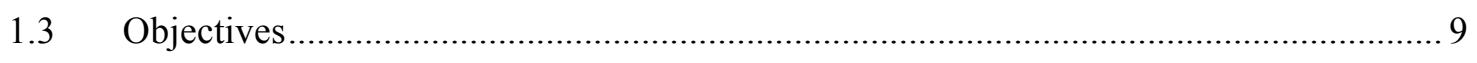

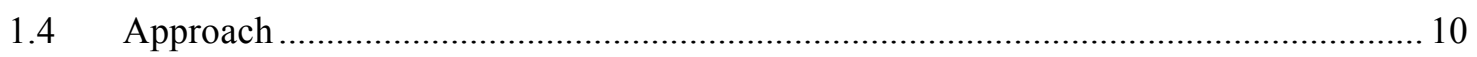

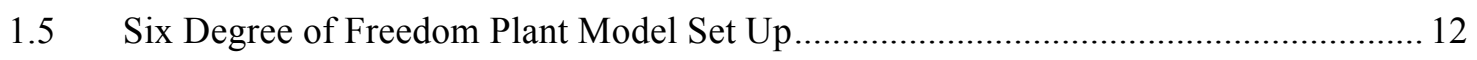

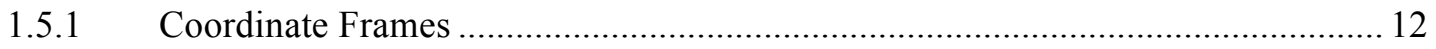

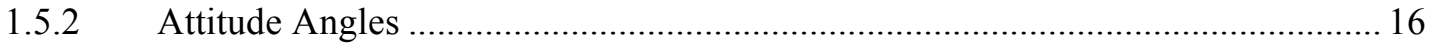

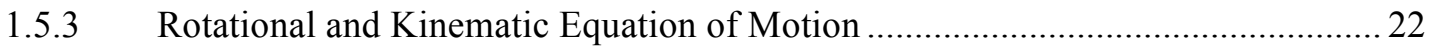

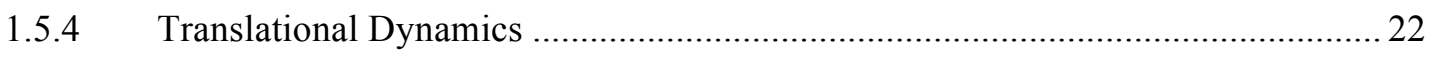

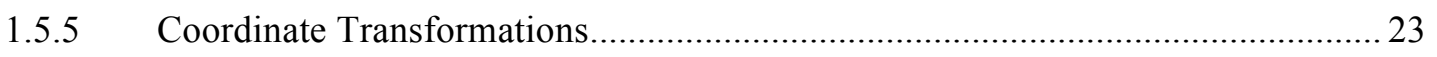

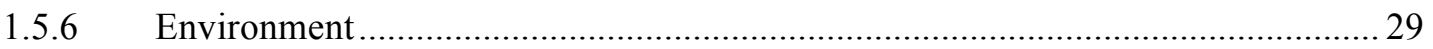

1.5.7 Reusable Launch Vehicle Parameters .................................................................... 31

1.6 Implementation of the Plant Model into Simulink ................................................... 36

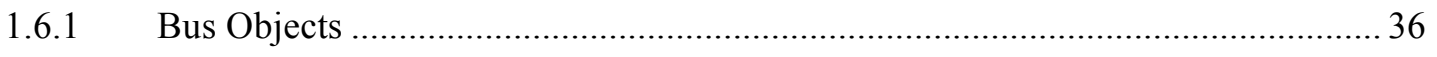

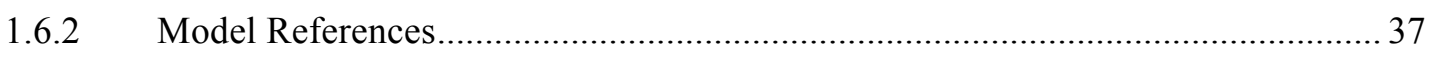

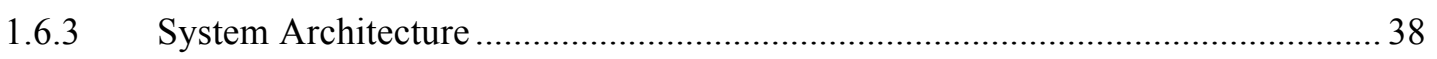

1.6.4 Vehicle Management Computer (VMC) ........................................................... 41

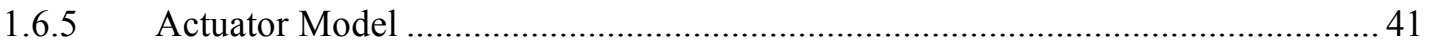

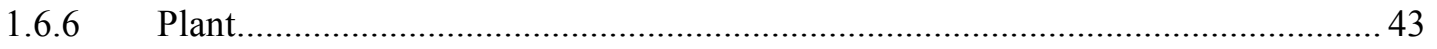

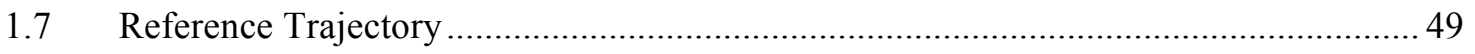

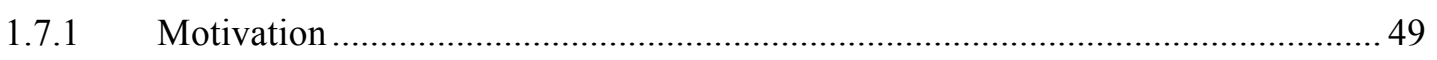

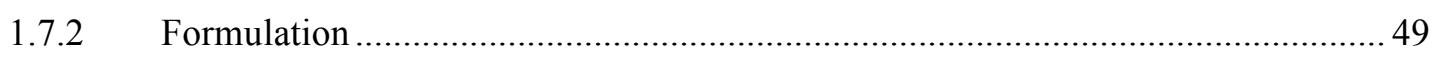

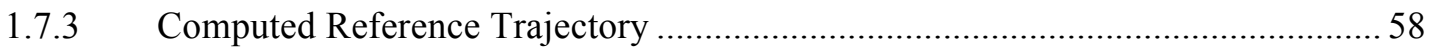

1.7.4 Implementation of the Reference Trajectory into Simulink ................................... 61

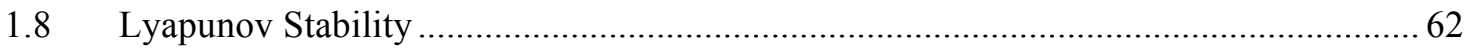

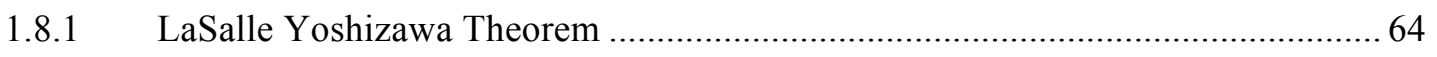

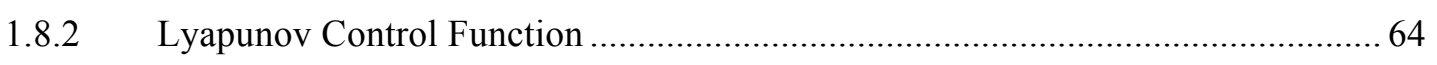

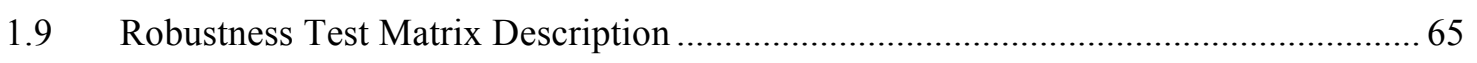




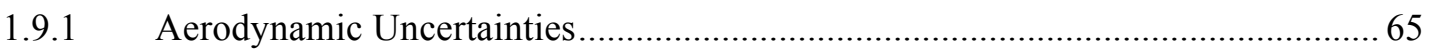

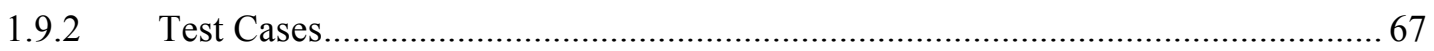

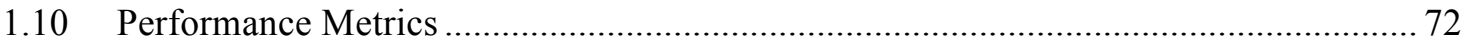

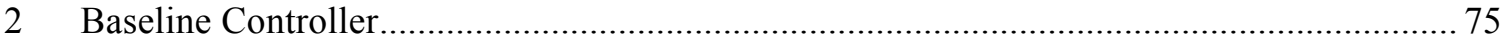

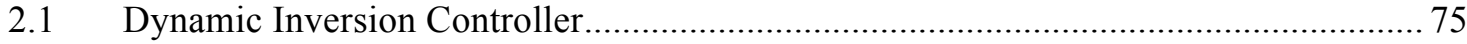

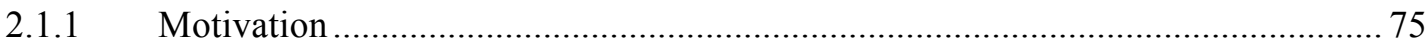

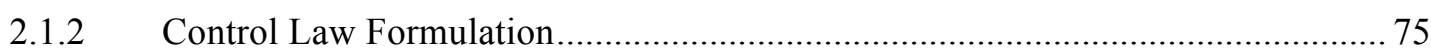

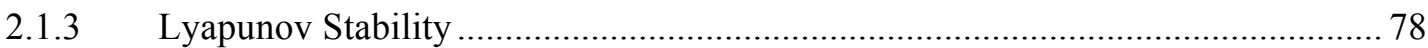

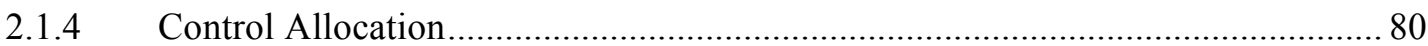

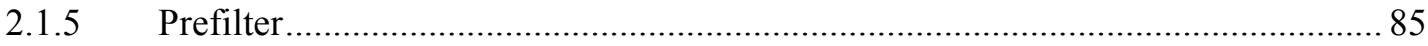

2.1.6 Dynamic Inversion Controller Implementation into Simulink ............................. 86

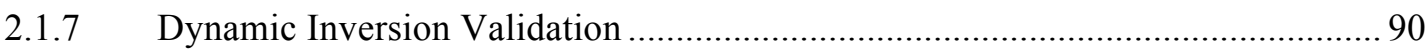

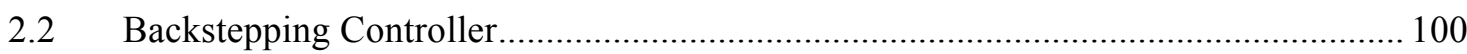

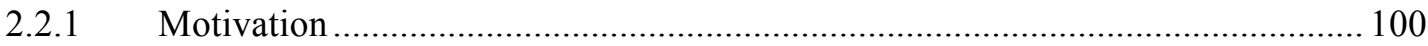

2.2.2 Backstepping Formulation with Control Lyapunov Functions ............................ 100

2.2.3 Backstepping Control Law Derivation.............................................................. 104

2.2.4 Backstepping Controller Validation and Performance ....................................... 129

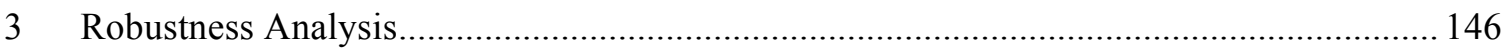

3.1 Body Rate Doublet Robustness Analysis...................................................................... 147

3.2 Trajectory Tracking Robustness Analysis ............................................................ 181

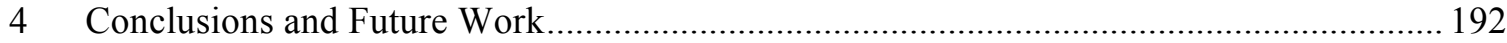

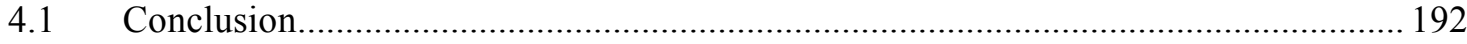

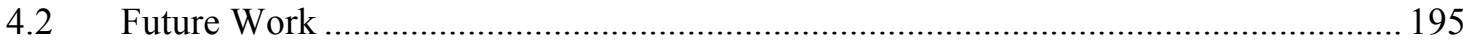

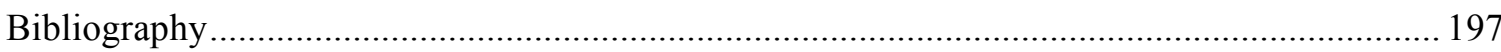

Appendix A: Approach and Landing Trajectory Propagation Scripts ....................................... 201

Appendix B: Vincenty Function Used to Compute Crosstrack ...................................................2207

Appendix C: Pseudo Control Computation for Backstepping Side slip and Bank angle Dynamics 


\section{List of Tables}

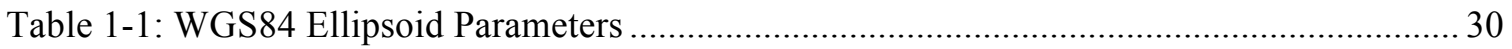

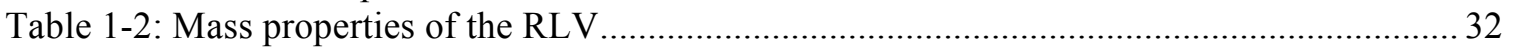

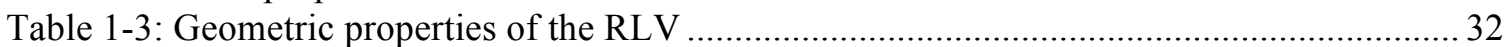

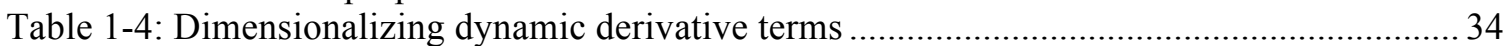

Table 1-5: Control Deflection Maximum and Minimum Limits. ................................................. 42

Table 1-6: Approach and Landing Interface and Touchdown Conditions....................................5 58

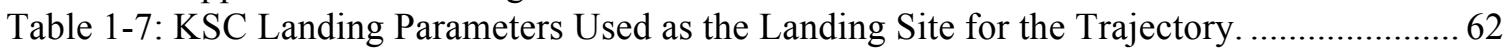

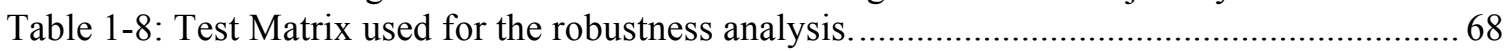

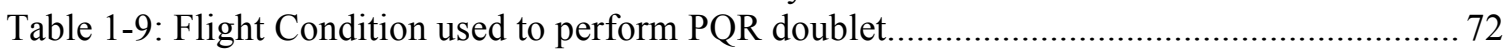

Table 1-10: Figures of merit for the controller tracking performance....................................... 73

Table 3-1: Maximum steady state error for roll rate doublet with $30 \%$ plant uncertainty........... 152

Table 3-2: Maximum steady state error for roll rate response with $40 \%$ plant uncertainty......... 153

Table 3-3: Standard deviation for roll rate response with 50\% plant uncertainty. ....................... 156

Table 3-4: Maximum steady state error for roll rate response with $60 \%$ plant uncertainty......... 158

Table 3-5: Maximum steady state error for pitch rate response with $30 \%$ plant uncertainty...... 160

Table 3-6: Maximum steady state error for pitch rate response with 40\% plant uncertainty. ..... 164

Table 3-7: Maximum steady state error for pitch rate response with $50 \%$ plant uncertainty...... 166

Table 3-8:Maximum steady state error for pitch rate response with $60 \%$ plant uncertainty....... 168

Table 3-9: Maximum steady state error for yaw rate response with $30 \%$ plant uncertainty. ...... 171

Table 3-10: Maximum steady state error for yaw rate response with $40 \%$ plant uncertainty. .... 175

Table 3-11: Maximum steady state response for yaw rate doublet with 50\% plant uncertainty. 178

Table 3-12: Maximum steady state error for yaw rate response with $60 \%$ plant uncertainty. .... 180

Table 3-13: Controller performance for the landing trajectory tracking with 10\% plant uncertainty. 


\section{List of Figures}

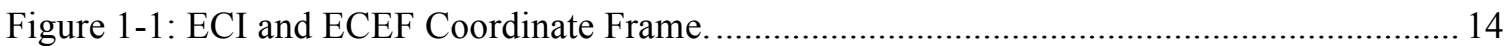

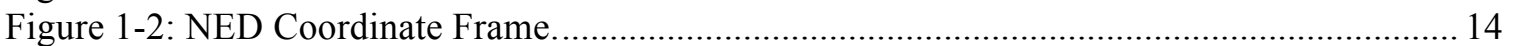

Figure 1-3: Geodetic versus Geocentric latitude measurements................................................ 15

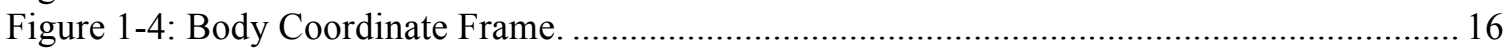

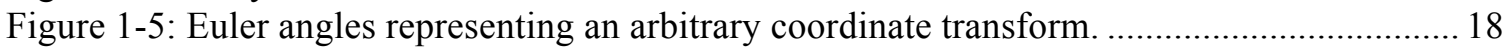

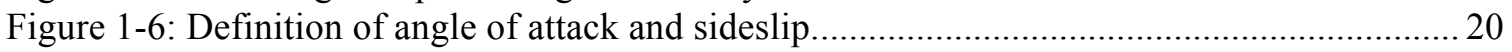

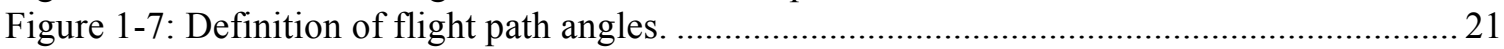

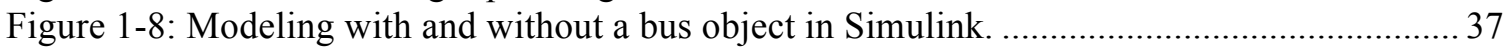

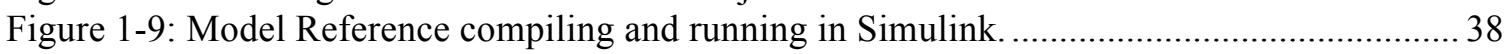

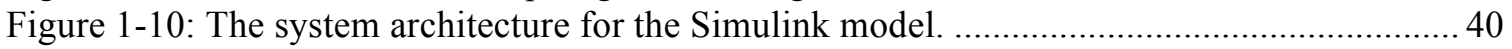

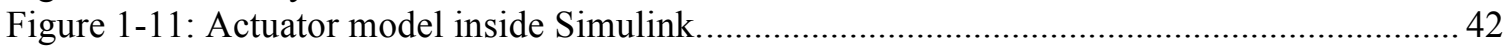

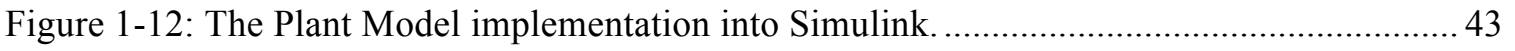

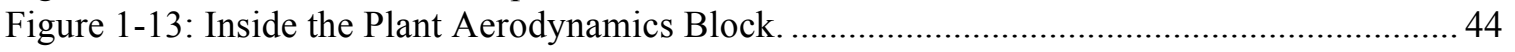

Figure 1-14: Simulink implementation of forces and moments computation from the bare

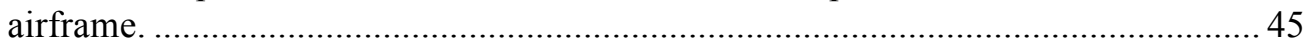

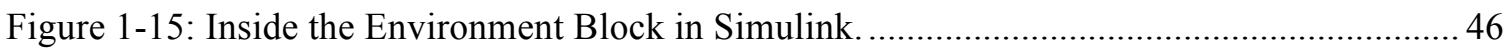

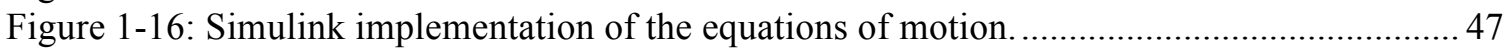

Figure 1-17: Translational and Rotational Dynamic Models in Simulink ................................. 48

Figure 1-18: Approach and Landing altitude profile with respect to downrange. .........................50

Figure 1-19: Steps to compute the approach and landing trajectory ............................................5 57

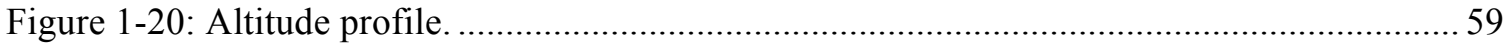

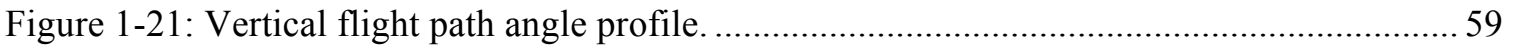

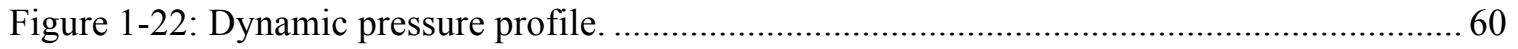

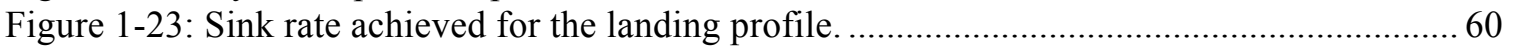

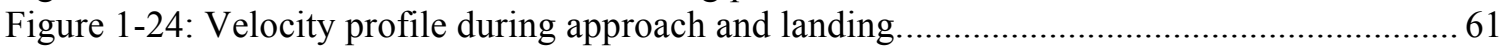

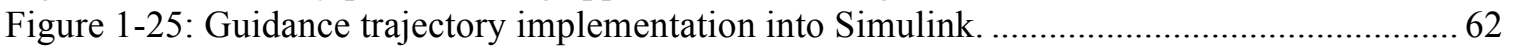

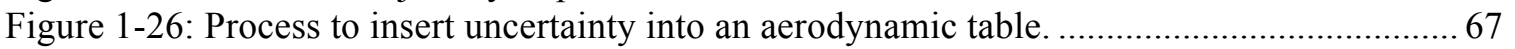

Figure 1-27: Sample mean convergence to zero for the $+/-30 \%$ Uncertainty Case........................69

Figure 1-28: Body rate doublet commands fed into the dynamic inversion controller. ................. 70

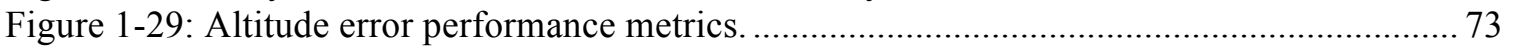

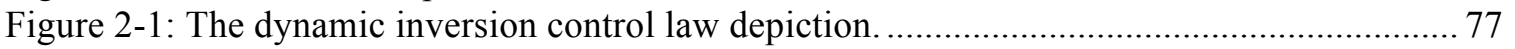

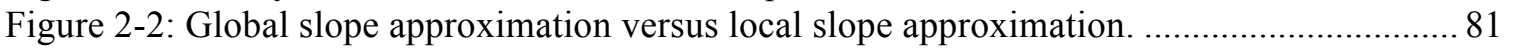

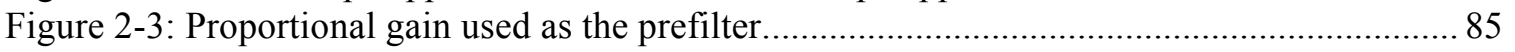

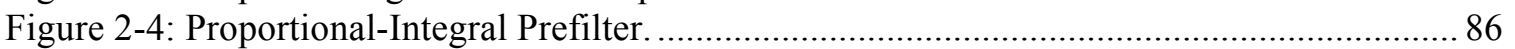

Figure 2-5: Dynamic Inversion control Law Implementation into Simulink. .............................. 87

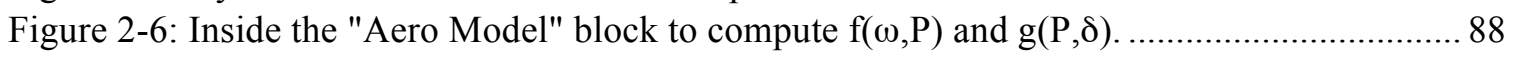

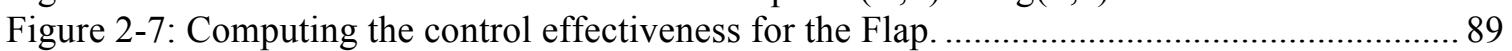

Figure 2-8: The control allocator to compute the control surface deflection commands. .............90

Figure 2-9: Prefilter comparison pitch rate doublet response. .................................................. 91

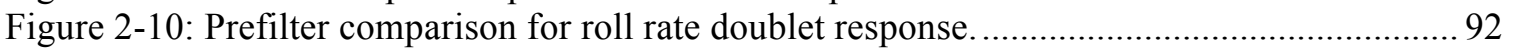

Figure 2-11: Prefilter comparison for a yaw rate doublet response. .......................................... 92

Figure 2-12: Baseline controller performance for roll rate doublet input.................................. 94

Figure 2-13: Desired acceleration command used by the control allocator................................. 95

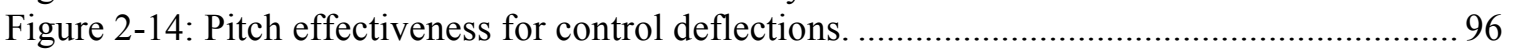




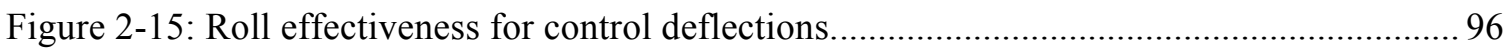

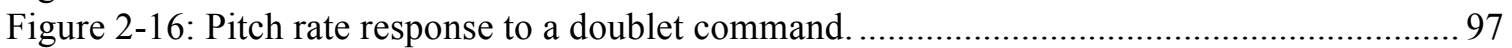

Figure 2-17: Control effectiveness computation can vary drastically at a break point................. 98

Figure 2-18: Yaw rate response to a doublet command............................................................. 99

Figure 2-19: Functional architecture for the longitudinal backstepping controller. ..................... 104

Figure 2-20: Simulink implementation of the pitch-axis backstepping controller. ...................... 112

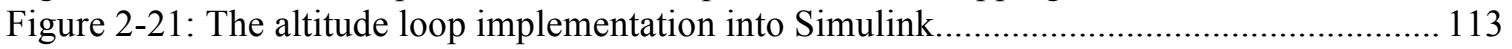

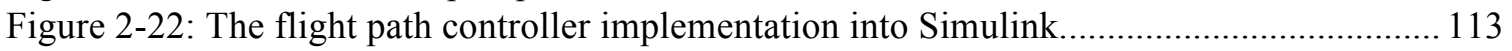

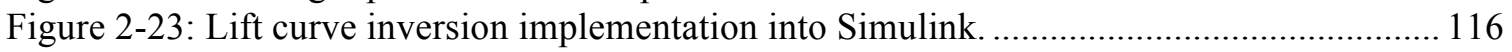

Figure 2-24: Vehicle state definition used in the pitch axis backstepping controller. ................. 117

Figure 2-25: Angle of attack control law implementation into Simulink................................. 117

Figure 2-26: Functional architecture for the lateral-directional axis backstepping controller..... 118

Figure 2-27: Crosstrack error definition using parameters computed from inverse vincenty. .... 119

Figure 2-28: Definition of heading command developed from the crosstrack error................... 120

Figure 2-29: Lateral-directional axis backstepping controller implementation........................... 127

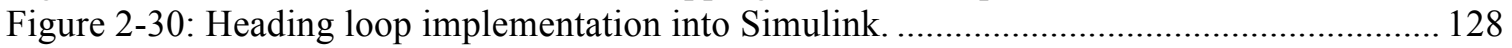

Figure 2-31: Bank angle and side slip angle control law implementation................................. 129

Figure 2-32: Angle of attack tracking using the backstepping controller.................................. 130

Figure 2-33: Flight path angle doublet command response and the associated angle of attack

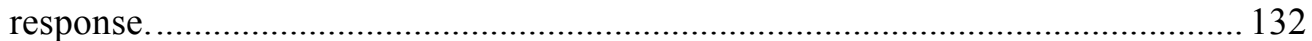

Figure 2-34: Flight path angle capture test case with a positive step input command.................. 133

Figure 2-35: Altitude tracking for a ramp input with the corresponding flight path

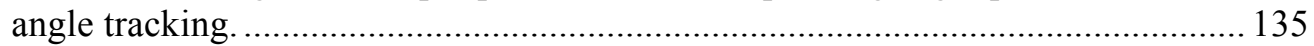

Figure 2-36: Bank angle doublet response and the corresponding roll rate response.................. 137

Figure 2-37: The side slip angle during the bank angle doublet maneuver. ............................... 138

Figure 2-38: Heading angle response to a doublet command................................................ 139

Figure 2-39: Crosstrack error increases even if the heading command tracks within

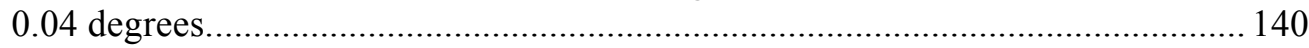

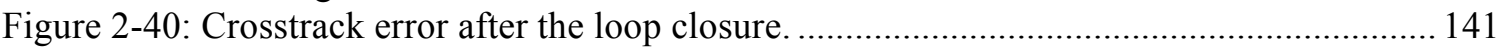

Figure 2-41: Altitude error and the sink rate for tracking the approach and landing trajectory. . 142

Figure 2-42: Crosstrack error and vehicle heading tracking.................................................... 142

Figure 2-43: Flight path angle and angle of attack tracking during the approach and landing phase.

Figure 2-44: Body rate response for the first 25 seconds of the trajectory................................. 144

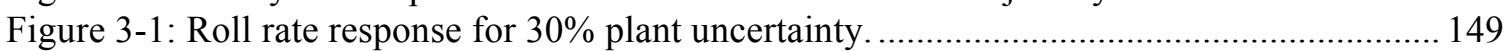

Figure 3-2: Standard deviation of roll rate responses for 30\% plant uncertainty. ....................... 151

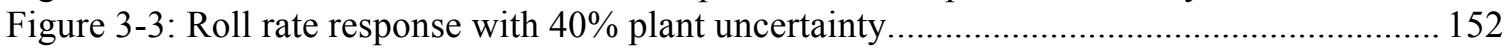

Figure 3-4: Standard deviations for roll rate response with $40 \%$ plant uncertainty................... 153

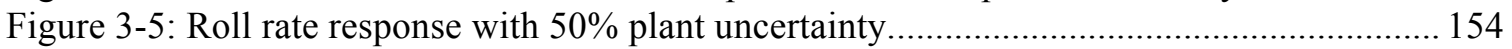

Figure 3-6: Standard deviation for roll rate responses with 50\% plant uncertainty.................... 155

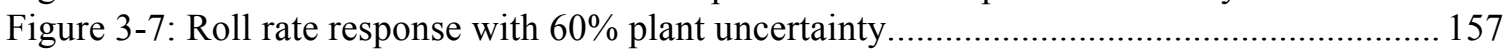

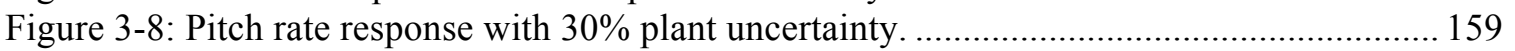

Figure 3-9: Standard deviation for pitch rate response with 30\% plant uncertainty.................... 161

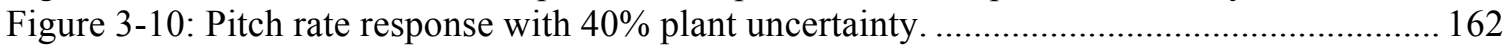

Figure 3-11: Standard deviation for pitch rate response with 40\% plant uncertainty .................. 163

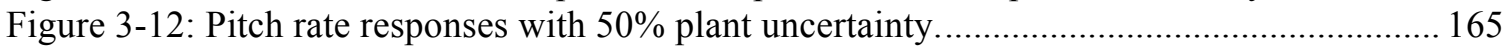

Figure 3-13: Standard deviation for pitch rate response with 50\% plant uncertainty.................. 166

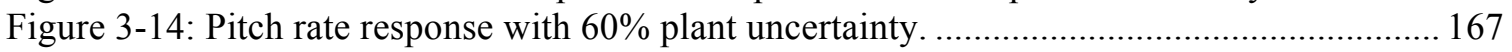


Figure 3-15: Standard deviation for pitch rate response with $60 \%$ plant uncertainty.................. 168

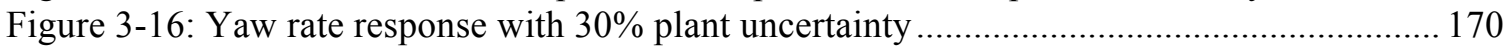

Figure 3-17: Standard deviation for yaw rate response with 30\% plant uncertainty.................. 171

Figure 3-18: Yaw rate response with 40\% plant uncertainty.................................................. 173

Figure 3-19: Standard deviation for yaw rate response with $40 \%$ plant uncertainty................... 174

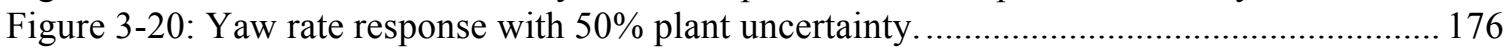

Figure 3-21: Standard deviation for yaw rate response with 50\% plant uncertainty................... 177

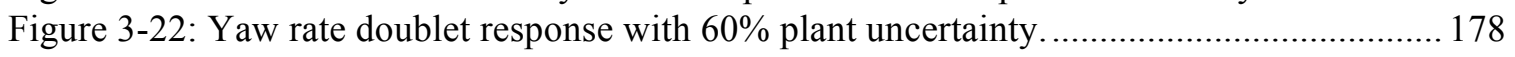

Figure 3-23: Standard deviation for yaw rate responses with $60 \%$ plant uncertainty. ................ 179

Figure 3-24: Altitude error and flight path angle profile with $10 \%$ plant uncertainty................ 183

Figure 3-25: Zoomed in view of the flight path angle profile for $10 \%$ plant uncertainty............ 185

Figure 3-26: Sink rate at touchdown and side slip angle profile for $10 \%$ uncertainty................. 187

Figure 3-27: Crosstrack and heading profile for $10 \%$ uncertainty case ..................................... 189

Figure 3-28: Standard deviation for altitude error, sink rate, crosstrack error, and heading for

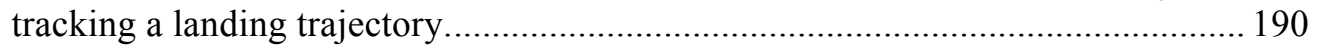

Figure 3-29: Sink rate at touchdown and flight path angle profile with $20 \%$ plant uncertainty.. 191 


\section{Nomenclature}

\begin{tabular}{|c|c|c|c|}
\hline$a$ & Acceleration vector / Speed of sound & $\boldsymbol{P}$ & Roll rate / position vector \\
\hline$a_{E}$ & Earth's semimajor axis & $\mathbf{Q}$ & Pitch rate \\
\hline ALTV & Approach and Landing Test Vehicle & $q$ & Quaternion element \\
\hline \multirow[t]{2}{*}{ B } & \multirow{2}{*}{$\begin{array}{l}\text { Control derivative of body acceleration with } \\
\text { respect to control deflections }\end{array}$} & Qbar & Dynamic pressure \\
\hline & & QEG & Quasi-equilibrium glide \\
\hline$b$ & span & $\dot{\boldsymbol{q}}$ & Quaternion element rate \\
\hline$b_{E}$ & Earth's semiminor axis & $\dot{\bar{q}}$ & Time derivative of dynamic \\
\hline $\boldsymbol{C A}$ & Axial force coefficient & & pressure \\
\hline$C G$ & Center of gravity & $\mathbf{R}$ & Yaw rate / Radius of the pull up \\
\hline$C D$ & Drag force coefficient & & phase \\
\hline $\boldsymbol{C L}$ & Lift force coefficient & RLV & Reusable Launch Vehicle \\
\hline $\boldsymbol{C l}$ & Roll moment coefficient & $s$ & Ground track \\
\hline CLF & Control Lyapunov function & Sref & Reference area \\
\hline $\mathrm{Cm}$ & Pitch moment coefficient & SSTO & Single stage to orbit \\
\hline$C N$ & Normal force coefficient & $\boldsymbol{u}$ & Velocity element in the $\mathrm{x}-$ \\
\hline Cn & Yaw moment coefficient & & direction \\
\hline $\boldsymbol{C Y}$ & Side force coefficient & UTC & Coordinated Universal Time \\
\hline$D$ & Drag force & $\mathbf{V}$ & Velocity vector/ Lyapunov \\
\hline DCM & Direction cosine matrix & & function \\
\hline DoF & Degree of freedom & $v$ & Velocity element in the $y-$ \\
\hline ECEF & Earth centered earth fixed & & direction \\
\hline ECI & Earth centered inertial & VMC & Vehicle Management Computer \\
\hline EELV & Evolved Expendable Launch Vehicle & $w$ & Velocity element in z-axis \\
\hline EML & Embedded Matlab & $\mathbf{X}$ & Force in the $\mathrm{x}$-axis direction \\
\hline$F$ & Force vector & $x$ & Body coordinate $\mathrm{x}$-axis \\
\hline$f$ & Earth flattening parameter & Xtrack & Crosstrack \\
\hline$e$ & Eccentricity & $\mathbf{Y}$ & Side force \\
\hline$g$ & Acceleration due to gravity/ & $y$ & Body coordinate $y$-axis \\
\hline & Control dependent acceleration effectiveness & $\mathbf{Z}$ & Force in the $\mathrm{z}$-axis direction \\
\hline GAS & Globally asymptotically stable & & \\
\hline $\mathbf{G}_{\mathbf{B A E}}$ & Moment contribution from the bare airframe & $\alpha$ & Angle of attack \\
\hline & (wing-body) & $\beta$ & Side slip angle \\
\hline $\mathbf{G}_{\text {。 }}$ & Moment derivative with respect to control & $\chi$ & Heading angle \\
\hline & deflection & $\delta$ & Control deflection \\
\hline $\mathbf{G}^{\mathrm{e}}$ & Gravitational attraction vector & $\varepsilon$ & Intercept term to correct linear \\
\hline GM & Earth's gravitational constant & & approximation \\
\hline GMST & Greenwich mean sidereal time & $\phi$ & Euler roll angle \\
\hline $\boldsymbol{h}$ & altitude & $\gamma$ & Vertical flight path angle \\
\hline$\dot{H}$ & Sink rate & $\lambda$ & Longitude \\
\hline $\boldsymbol{I}$ & Moment of inertia & $\mu$ & Bank angle \\
\hline $\boldsymbol{L}$ & Roll moment / Lift force & $\varphi_{c}$ & Geocentric latitude \\
\hline $\boldsymbol{L}_{\alpha}$ & Lift curve slope with respect to angle of attack & $\varphi$ & Geodetic latitude \\
\hline $\boldsymbol{M}$ & Moment vector / Pitch moment & $\theta$ & Euler pitch angle \\
\hline$m$ & Mass & $\theta_{\mathrm{g}}$ & Greenwich mean sidereal time \\
\hline mac & Mean aerodynamic chord & $\theta_{\mathrm{s}}$ & Sidereal time \\
\hline MET & Mission elapsed time & $\rho$ & Density \\
\hline$N$ & Yaw moment & $\omega$ & Angular velocity \\
\hline NED & North-east-down frame & & Earth's rotation vector \\
\hline ORS & Operationally responsive space & $\omega_{\mathrm{B}}$ & Euler yaw angle \\
\hline OTV & Orbital Test Vehicle & $\psi$ & Euler yaw angle \\
\hline
\end{tabular}




\section{Subscripts}

\begin{tabular}{|c|c|}
\hline $2 / 1$ & $\begin{array}{l}\text { Coordinate transformation from frame } 1 \\
\text { to frame } 2 \text { (for DCM) }\end{array}$ \\
\hline actual & Vehicle response \\
\hline ALI & Approach and landing interface \\
\hline b & Body coordinates \\
\hline BAE & $\begin{array}{l}\text { Contribution from the bare airframe } \\
\text { (wing-body) }\end{array}$ \\
\hline b/ECI & $\begin{array}{l}\text { Coordinate transform from ECI frame to } \\
\text { the body frame }\end{array}$ \\
\hline $\boldsymbol{C G}$ & Center of gravity \\
\hline cmd & Commanded \\
\hline D & $\begin{array}{l}\text { Down axis in the North-East-Down } \\
\text { frame }\end{array}$ \\
\hline$d$ & Derivative gain \\
\hline des & Desired \\
\hline $\mathbf{E}$ & East axis in the North-East-Down frame \\
\hline$E C E F$ & Earth centered earth fixed coordinates \\
\hline ECI & Earth centered inertial coordinates \\
\hline $\mathbf{F}$ & Flap \\
\hline flare & Flare phase \\
\hline$i$ & Integral gain \\
\hline Lin & Left Inboard \\
\hline Lout & Left Outboard \\
\hline $\mathbf{L R}$ & Left Rudder \\
\hline $\mathbf{N}$ & $\begin{array}{l}\text { North axis in the North-East-Down } \\
\text { frame }\end{array}$ \\
\hline NED & North-East-Down coordinate frame \\
\hline$p$ & Proportional gain \\
\hline pu & Circular pull up phase \\
\hline ref & $\begin{array}{l}\text { Referenced from the aerodynamic } \\
\text { reference }\end{array}$ \\
\hline $\boldsymbol{r e l}_{b}$ & Relative to the body coordinate \\
\hline Rin & Right Inboard \\
\hline RR & Right Rudder \\
\hline$s$ & Stability axis \\
\hline SGS & Steep glide slope phase \\
\hline$x$ & Element in the $\mathrm{x}$-axis direction \\
\hline$y$ & Element in the $y$-axis direction \\
\hline$z$ & Element in the z-axis direction \\
\hline
\end{tabular}




\section{Introduction}

\subsection{Background on Reusable Launch Vehicles}

The U.S. relies heavily on space systems as technology advances. We developed advanced satellites for various functions such as remote sensing, communications, navigation, imagery, and missile warnings. These technologies are needed for both scientific research and national security. As a result, the Department of Defense and NASA has been working towards responsive access to space ${ }^{33}$. They need to meet commercial and military demands for safer, less expensive, and more reliable access to space than currently operational launch vehicles, which include EELVs and the Space Shuttle System. Atlas V and Delta IV from the EELV program have proven to successfully put payloads into orbit with failure rates between 1 and 10 percent. However, the EELVs come at a cost; their costs to launch have been escalating anywhere between $\$ 120$ million and $\$ 165$ million per flight ${ }^{30}$. The Air Force is planning to phase out and replace the EELVs in 2030 due to their rising costs ${ }^{44}$. NASA has the partially reusable Space Shuttle to provide access to space for research, maintaining and supplying the International Space Station. The Space Shuttle System costs between $\$ 400$ million and $\$ 1$ billion for every flight ${ }^{43}$. It depends heavily on the winds and the weather to be able to launch and land; often times the schedules are delayed due to bad weather. The Space Shuttle was supposed to be a transportation system to open up space with routine flights. However, the long turnaround times and the cost to get it ready for flight prevent the shuttle from flying frequently. Moreover, two orbiters have been

destroyed in 130 missions, giving a failure rate of 1 in every 65 missions $^{31}$; imagine if commercial flights had the same failure rate? The number of customers will decline drastically. The Space Shuttle will be retired in 2011 with the last scheduled launch to be in February $24^{26}$. 
Thus, EELVs and the Space Shuttle need to be replaced by more advanced technology and architecture to reduce operational and maintenance cost and provide quick turnaround times between flights to deal with new requirements derived from rapidly changing U.S. technology and support needs ${ }^{11}$. The Air Force and NASA have identified reusable launch vehicle as the solution for on-demand access to space while cutting current EELV launch costs by $50 \%$ for 100 flights, replacing engines every 10 years ${ }^{44}$.

There have been many programs used to research and develop technology to support hypersonic and space flight challenges in the last 50 years: the first Aerospaceplane program and Dyna-Soar/X-20 program (late 1950s-early 1960s); X-15 hypersonic and X-24 lifting body flight test programs (late 1950s through early 1970s); Advanced Military Space Flight Capability (AMSC), Transatmospheric Vehicle (TAV), and Military Aerospace Vehicle (MAV) concept and mission studies (early 1980s); the Copper Canyon airbreathing single-stage-to-orbit (SSTO) feasibility assessment and the National Aerospace Plane (NASP) program (1984-1992) ${ }^{39}$. More recently in the last 10 years, NASA developed the Space Launch Initiative program in 1996 with the objective of increasing safety and reliability and reducing costs associated with developing, building, and maintaining the next generation of space launch vehicles. NASA's goal was to reduce launch costs from $\$ 10,000$ per pound to orbit to $\$ 1,000$ per pound ${ }^{33}$. The program ended with the cancellation of the X-33 and the X-34 in $2001^{33}$.

The X-33 is a suborbital, unmanned, technology demonstrator for a single-stage-to-orbit reusable launch vehicle (SSTO RLV) concept. NASA awarded Lockheed Martin to design, build, and fly the X-33. It was supposed to be a $1 / 3$ scale prototype of the fully operational VentureStar ${ }^{4}$. NASA intended the VentureStar to be the first commercially operated launch vehicle, but was never developed because of the cancellation of the X-33 program. NASA 
believed that removing the "staging" of the launch vehicle would provide more reliable and safer launch vehicle. The X-33 program objective was to flight test a range of technologies for a SSTO launch vehicle: composite cryogenic fuel tanks for liquid hydrogen, the aerospike engine, lifting body aerodynamics, and autonomous flight controls. It was designed to launch vertically, reach an altitude of 60 miles and velocities greater than Mach 13, land horizontally, and demonstrate a seven-day turnaround ${ }^{32}$. The failure of the composite liquid hydrogen tank ultimately led to the cancellation of the program in $2001^{4}$. However, Lockheed continued with the research vehicle and recently achieved success with several flight tests conducted between 2008 through 2009 . The last successful flight was out of the Spaceport America in New Mexico on October 10, $2009^{10}$.

The X-34 was a low-cost suborbital testbed to demonstrate reusable launch vehicle technologies. NASA awarded Orbital Sciences to develop and flight test the X-34. The X-34 is an unmanned suborbital vehicle capable of reaching Mach 8 and performing 25 flight tests per year $^{34}$. It was supposed to be dropped from the L-1011 carrier aircraft and fly a predetermined flight profile, powered, before making an autonomous approach and landing on a conventional runway unpowered ${ }^{34}$. However, it was cancelled before any flight tests and still resides in storage at the Edwards Air Force Base ${ }^{34}$.

The Air Force has been looking into reusable launch vehicle to provide reconnaissance, global strike, global transport, space control, and satellite servicing. These vehicles could be designed to rendezvous with satellite to refuel them, or replace failed solar arrays using a robotic $\operatorname{arm}^{11}$. Hypersonic space vehicles have the potential to support advanced capabilities to the warfighter. They could deliver quick, long range response to any threat before they impact the U.S. Space provides the platform to enhance weapons delivery, global communications, and 
intelligence work in order to protect U.S. assets and interrupt enemy operations. Therefore, rapid turnaround is needed to support time-critical missions to support the warfighter ${ }^{11}$. Operationally Responsive Space (ORS) calls for a launch vehicle with a turnaround time between 24 to 48 hours, with an 8 hour call up time, available for all weather, at three times the lower cost than current launch vehicles ${ }^{22}$. The Air Force has funded the X-40, X-37, and other reusable launch vehicle research projects to help advance the technology necessary for ORS.

The Air Force's X-40, also known as the Space Maneuver Vehicle, is designed and built by Boeing Phantom Works. It is an unmanned and unpowered vehicle served as the technology demonstrator for the X-37 Future-X Reusable Launch Vehicle project. It is $85 \%$ scale to the X-37 and built with a similar shape as the X-37, but without the advanced TPS material. The X-40 was successfully able to demonstrate glide capabilities of the X-37's aerodynamic design and verified the guidance system ${ }^{38}$. The first glide test on August 11, 1998 proved to be successful. The X-40 was dropped at an altitude of 9,000 feet from the UH-60 Black Hawk helicopter and autonomously landed after performed a glide maneuver simulating the approach and landing of an orbital vehicle. The first flight was at NASA's Dryden Flight Research Center where the vehicle glided down to land from 15,000 feet, attached by a tether line to the $\mathrm{CH}-47^{40}$. It was able to perform seven successful flight tests up to May 2001 to reduce risks before the X-37 flight test.

The X-37, designed and built by Boeing, began as a NASA project back in 1999, but transferred to the Defense Advanced Research Project Agency (DARPA) in 2004 ${ }^{28}$. The X-33 and the X-34 were intended as reusable technology demonstrators at lower altitude and speeds, while the X-37 would test the orbital and re-entry phases of flight. The X-37 program objectives include space experimentation, risk reduction and a concept of operations development for the reusable space vehicle technologies. NASA wanted to develop two vehicles under the X-37 
program: an Approach and Landing Test Vehicle (ALTV) and an Orbital Test Vehicle (OTV) ${ }^{35}$. The ALTV vehicle successfully performed a series of captive carry and free flight tests by September $2006^{28}$. With the success of the ALTV, X-37B OTV abroad an Atlas V rocket was launched into low Earth orbit on April 23, 20109. Solar panels were deployed during the 227 days orbit to charge batteries for electrical power. The unmanned OTV evaluated the guidance, navigation, thermal protection, and unmanned operations in orbit, re-entry, and landing to provide insight on the technologies used to provide operationally responsive space. It was the first unmanned vehicle to autonomously de-orbit, reenter, and successfully land at Edwards AFB on December 3, $2010^{37}$. The X-37 was expected to experience speeds of up to Mach 25 and have a turnaround time between 10 and 15 days or less. The objective of the OTV was to demonstrate inexpensive and fast turnaround times. The second orbital flight is scheduled between the March and April 2011 time period. The second flight will expand the flight envelope and increase the orbital cross range, and test its ability to land in stronger crosswinds ${ }^{37}$.

More recently, the Air Force is planning to begin technology development for two versions of the reusable booster system (RBS), intended to replace EELVs beyond 2025. The first type is a reusable first stage with an expendable second stage for medium-lift missions, the second is a fully reusable first and upper stage for heavy-lift missions. The RBS will launch vertically, place the upper stage into orbit, perform a powered jet-back maneuver to reverse the booster's flight path 180 degrees, back toward the launch site, and do an unpowered re-entry and approach and landing ${ }^{44}$. The technology development will begin with the Air Force Research Laboratory's (AFRL) RBS Pathfinder program. The pre-solicitation notice calls for a subscale Xplane planned to fly in 2013 to demonstrate the "rocket-back" return-to-launch-site (RTLS) maneuver using different methods. It will serve as a research platform for aerodynamic loads at high altitudes and high angles of attack and sideslip required to do this "rocket-back" maneuver. 
The follow on to the Pathfinder will be the "moderately large-scale" RBX demonstrator, representative of the operational system. The Pathfinder will be 15 feet long, while the RBX is expected to be 50 to 60 feet long ${ }^{44}$. The purpose of all of these programs is to take the concept of the reusable launch vehicle (RLV) "to a technology readiness level of 6 , ready to enter full scale development," making the feasibility of the RBS concept a reality ${ }^{44}$.

Guidance and control technology is recognized as an important aspect of the Air Force goal of reliable, low cost operations into space. AFRL has funded Northrop Grumman for the Integrated Adaptive Guidance and Control for Ascent program as well as the follow-on contract, the Full Envelope Adaptive Guidance and Control for Unmanned RLV. Both contracts were created to specifically develop adaptive guidance and control algorithms used to increase survivability and maximize performance of the launch vehicle.

\subsection{Motivation}

Reusable launch vehicles (RLVs) maneuver over a flight envelope ranging from Mach 0 to over Mach 8 and altitudes from 0 to over 500,000 feet. Such a wide flight envelope creates challenges in determining dynamics of a vehicle especially at hypersonic mach numbers. Moreover, high speed vehicles often contain nonlinearities in aerodynamic forces and moments. A large dynamic range and nonlinear aerodynamics pose difficulty in designing a flight controller for a RLV using classical gain scheduling techniques ${ }^{8}$.

Flight controller algorithms for the space shuttle and expendable launch vehicles currently use classical gain scheduling techniques tuned to a specific nominal mission. Gain scheduling requires breaking up a nominal mission into multiple operational points and designing a controller after linearizing a plant about each of these points. A curve fit is done between each 
operational point to develop a controller for a full envelope flight ${ }^{3}$. A controller developed from a curve fitting procedure is only applicable to a set of linear models created for the particular launch vehicle configuration and nominal mission. Any changes in mass properties, aerodynamics, engine performance, or mission trajectory require rebuilding a set of linear models to obtain gain schedules for the new configuration and mission objectives ${ }^{18}$. Aside from the nominal trajectory, multiple abort trajectories have to be analyzed separately in the same manner to ensure adequate stability and performance in an event of an off-nominal case. Controller design for a space shuttle or an expendable launch vehicle becomes costly and time consuming using gain scheduling ${ }^{7}$.

The requirement for quick turnaround time and reliability may not be achievable for ORS if gain tuning has to be done for each nominal and off-nominal mission. Development of a reliable RLV will require flexibility to perform a large set of missions, rather than one nominal mission. Reduction in dependence on preflight planning reduces time and costs. Nonlinear control methodology offers a vehicle the ability to track a wide variety of trajectories with the same controller by dealing with complete nonlinear dynamics. Directly considering a vehicle's nonlinear dynamics eliminates a need for a trajectory dependent point designs like gain scheduling ${ }^{8}$. Some of the nonlinear control designs include dynamic inversion and backstepping.

Dynamic inversion contains a concept of cancelling out a plant's nonlinear dynamics and replacing it with desired linear dynamics through feedback linearization ${ }^{19}$. A disadvantage to feedback linearization is exact knowledge of a plant is required to cancel nonlinear dynamics, so small uncertainties cause loss of control if inexact cancellation introduces an unstable pole ${ }^{3}$. Therefore, an additional outer control loop is needed to add robustness to the dynamic inversion controller like backstepping. Backstepping is more flexible in handling uncertainties using 
Lyapunov functions to design controllers that guarantee stability and bounded tracking. The backstepping methodology keeps useful nonlinearities and cancels harmful nonlinearities that may reduce the system's stability ${ }^{19}$. The Lyapunov stability properties of the backstepping controller may provide the robustness necessary to use a dynamic inversion inner loop controller and a backstepping outer loop controller for a RLV to handle a range of missions.

Many papers have used dynamic inversion in conjunction with $\mathrm{H}$-infinity, or $\mathrm{Mu}-$ Synthesis, or Linear Quadratic Gaussian (LQG), or backstepping controllers to provide increase in performance and robustness to reduce developmental times of reusable launch vehicles and highly maneuverable fighters ${ }^{2}$. However, H-infinity, Mu-Synthesis, or LQG controllers are mathematically complex compared to backstepping. The H-infinity and LQG formulations require mathematical understanding to apply the optimization problem correctly. Mu-Synthesis and H-infinity often produces high order controllers that must be reduced for realistic applications $^{21}$. Backstepping controller formulation is straightforward once a set of equations for a system dynamics is put in strict or pure feedback form. Reference 5 and Reference 15 implements a backstepping outer loop controller with a dynamic inversion inner loop controller for a RLV. The General Dynamics total in-flight simulator (TIFS) research aircraft simulating the X-40A dynamics was drop tested and successfully flew the approach and landing profile ${ }^{15}$, proving a backstepping and dynamic inversion controller is feasible for RLV platforms.

A dynamic inversion controller stabilizes the inner loop by controlling body rates: roll, pitch, and yaw. The body rates are controlled through a dynamic inversion formulation cancelling a plant's nonlinear rotational dynamics, not translation dynamics. A control Lyapunov function (CLF) generated from rotational states proves the dynamic inversion controller is globally asymptotically stable (GAS). The stability proof assumes a plant could be inverted exactly, which 
may not be the case if there are aerodynamic uncertainties ${ }^{21}$. Moreover, the CLF only includes states from rotational dynamics. All states from a plant dynamics and all states used in feedback control have to be represented in a Lyapunov function in order to prove a whole system is stable. The backstepping controller implemented in this thesis was formulated by creating a CLF for a pitch-axis and a lateral directional-axis, independently, proving global asymptotic stability separately for each axis. Like the dynamic inversion formulation, the backstepping controller formulation proved stability for only parts of a system at a time. The formulation did not include states from the dynamic inversion controller. Technically, a whole system could not be proven GAS unless a control Lyapunov function contains all states of a system. Can stability be applied to a whole system if sum of the parts are proven to be stable? A robustness analysis will help verify whether this hypothesis is true.

Furthermore, both methods are model-based controllers and require accurate knowledge of plant dynamics. Aerodynamics of RLVs are often times difficult to predict accurately in flight regimes like high angles of attack and supersonic mach numbers. Uncertainties in flight regimes may affect controller stability and performance. Robustness analysis would help determine how much uncertainties could be tolerated before a vehicle is considered uncontrollable and inadequate.

\subsection{Objectives}

The focus of this thesis is to analyze and quantify the robustness of using a dynamic inversion inner loop with a backstepping outer loop controller by evaluating controller performance after introducing unmodeled plant uncertainties. The robustness analysis answers two questions: whether a stability of a whole system could be proven if sum of all of the parts of a 
system are proven GAS; whether using model-based controllers like backstepping and dynamic inversion together provide robustness to perturbations in a plant.

Northrop Grumman Corporations' (NGC) RLV configuration, designed to operate in a broad flight envelope, was sanitized to produce vehicle aerodynamic data for the plant model. A baseline backstepping and dynamic inversion controller was designed assuming perfect plant modeling. Perfect modeling means the on-board aerodynamic model used in the controller is the same as the aerodynamics used in the plant model. Robustness of the controller is analyzed by evaluating controller tracking for an unpowered approach and landing trajectory with and without plant uncertainties. Unmodeled plant uncertainties are implemented by adding perturbations to the NGC provided aerodynamic tables in the plant model, while keeping the original NGC aerodynamic tables in the controller. Dynamic inversion inner loop tracking and trajectory tracking are tested for aerodynamic uncertainties varying from $+/-10 \%$ to $+/-60 \%$ applied to static aerodynamics tables, control aerodynamics tables, separately, then both sets of tables together. Figures of merit are developed in order to quantify controller robustness by evaluating tracking performance for sets of command inputs.

In addition to building a baseline controller, a full nonlinear 6DoF simulation for the RLV and a reference trajectory is developed in order to fulfill the objective. A method developed by Kluever is used to generate a reference trajectory of an approach and landing phase ${ }^{27}$. The robustness analysis is conducted once these initial set ups were completed.

\subsection{Approach}

An approach used to analyze the robustness of the controller involves creating a $6 \mathrm{DoF}$ plant model first. A plant model is created in Simulink to simulate the RLV's nonlinear 
dynamics. Translational and kinematic equations of motion encompass a 6 DoF motion of a vehicle. One has to understand coordinate frames and attitude angles to use these equations to properly propagate a vehicle's orientation and position in time.

A test matrix containing different command inputs and different plant uncertainty types are developed to analyze robustness of the dynamic inversion and the backstepping controller together and separately. Various doublet commands are input into the controller to analyze inner loop tracking with a range of plant uncertainties. Reference trajectory tracking is used to evaluate robustness of the backstepping and dynamic inversion controller as a whole. Plant uncertainties are added to static aerodynamics and control surface aerodynamics separately and together to examine how each plant uncertainty type affects robustness of the controller for each set of commands in the test matrix. Figures of merit will be provided for each test to quantify tracking performance to evaluate robustness to each plant uncertainty type.

A landing trajectory is generated as part of an approach in order to evaluate how well the controller tracks a reference trajectory. An algorithm from Kluever is used to create this trajectory. The algorithm solves for a landing trajectory using vehicle aerodynamics so the resulting trajectory is within the physical energy constraints of a vehicle.

CLFs are used to derive globally asymptotically stable backstepping control laws and are also used to prove global asymptotic stability for a dynamic inversion control law formulation. Therefore, background information and theory behind control Lyapunov functions are explained in the Approach in order to understand the stability proofs for the baseline controller. 


\subsection{Six Degree of Freedom Plant Model Set Up}

A full 6 DoF plant model is developed in order to simulate RLV motion using a dynamic inversion and backstepping controller. A plant model includes aerodynamic nonlinearities and coupling between all three axes of rotation, roll, pitch, and yaw. The following sections present equations of motion developed for the $6 \mathrm{DoF}$ model under the assumption that the vehicle is a rigid, constant, point mass, flying over an oblate and rotating earth.

\subsubsection{Coordinate Frames}

Modeling flight dynamics consists of selecting a reference frame to derive the translational equation of motion to calculate acceleration, velocity, and position vectors in three dimensional space. RLVs travel at high altitudes and high velocities covering large areas of an earth's surface. This requires the equations of motion to be expressed in the earth centered inertial (ECI) frame as the "fixed" inertial frame to accurately model a vehicle's motion. The NGC provided RLV aerodynamic forces and moments are expressed in the body frame so they will have to be transformed into the inertial frame before computing acceleration. Acceleration in the inertial frame is integrated twice to get a vehicle's position. Another set of coordinate transformations is performed on a position vector to interpret a vector from inertial to navigational coordinates, which are easier to visualize and understand on an earth's surface. Five different coordinate frames: earth centered inertial (ECI), earth centered earth fixed (ECEF), north-east-down (NED), navigational (longitude, latitude, and altitude), and body coordinates are used to calculate a vehicle's motion from an ECI frame to a navigational frame. 


\subsubsection{Earth Centered Inertial Frame}

ECI frame is a non-accelerating frame of reference because it is considered "fixed" with respect to the stars, so it does not rotate with the earth. The origin is fixed at the center of the earth. The x-axis points outward in the direction of the vernal equinox, which is a point in space where the earth's equator intersects the plane of the earth's orbit around the $\operatorname{sun}^{29}$. The $z$-axis points north along the earth's rotational axis. The y-axis completes the right handed orthogonal system, as shown in Figure 1-1. Newton's second law, $F=m a$, is only applicable in an inertial frame so all vehicle forces and moments are transformed to the ECI frame ${ }^{29}$. Acceleration is integrated to get a vehicle's velocity and position in ECI frame.

\subsubsection{Earth Centered Earth Fixed Frame}

ECEF frame is a non-inertial frame that rotates with the earth, unlike the ECI frame, which stays fixed. Its origin is the same as the ECI frame, located at the center of the earth. The $\mathrm{x}$-axis points outward in the direction where the earth's equator interests the Prime Meridian. The z-axis points north along the earth's rotational axis like the ECI frame. The y-axis completes the right-handed orthogonal system ${ }^{29}$. The ECEF frame is an intermediary frame to transform velocity and position vectors from the ECI frame into the NED frame or into the navigational frame (longitude, latitude, and altitude). Figure 1-1 depicts how the ECEF frame differs from the ECI frame by a rotation about the z-axis. 


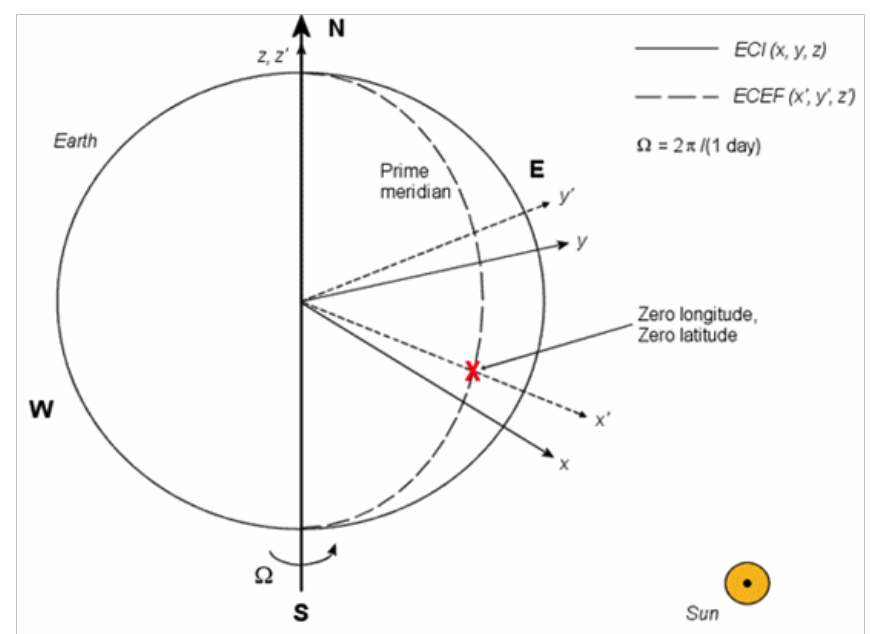

Figure 1-1: ECI and ECEF Coordinate Frame.

\subsubsection{North-East-Down Coordinate Frame}

NED frame, seen in Figure 1-2, is a vehicle carried system with its origin fixed at a vehicle's center of mass. The axes point along geographic directions of the earth's surface. The xaxis points north parallel to the earth's surface. The y-axis points along latitude lines in the east direction, parallel to the earth's surface. The z-axis points downward in an opposite direction of an outward normal to the earth's surface ${ }^{29}$. The NED frame is used to calculate vehicle's flight path angles like $\gamma$ (vertical flight path angle) and $\chi$ (heading angle) to follow a reference trajectory.

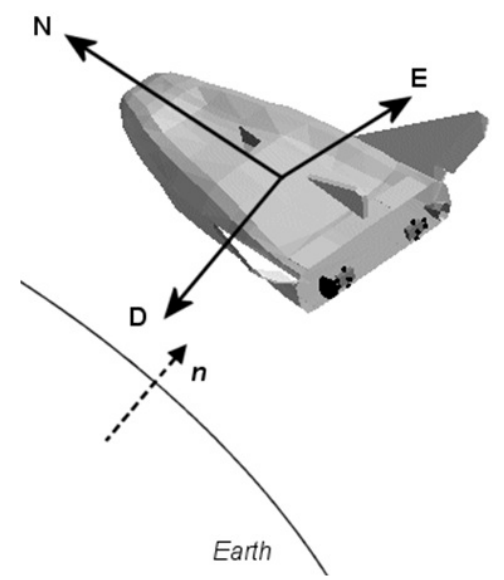

Figure 1-2: NED Coordinate Frame. 


\subsubsection{Navigational Frame}

The navigational frame uses the WGS84 Geoid to transform its coordinates from the ECEF frame in Cartesian coordinates to coordinates on an ellipsoid. Elements in a navigational frame are the geodetic states: geodetic longitude, latitude and altitude that physically interpret a vehicle's position on earth. This frame allows a vehicle to be mapped onto an earth's surface so waypoint guidance could be used to track a reference trajectory.

Geocentric coordinates are similar to geodetic coordinates except its reference is from a center of an ellipsoid. The longitude measurement is the same, but the latitude measurements differ. Geodetic latitude, $\varphi$, is the angle between the normal of the ellipsoid and the plane of the equator, while geocentric latitude, $\varphi_{c}$, is measured from the center ${ }^{29}$ as seen in Figure 1-3.

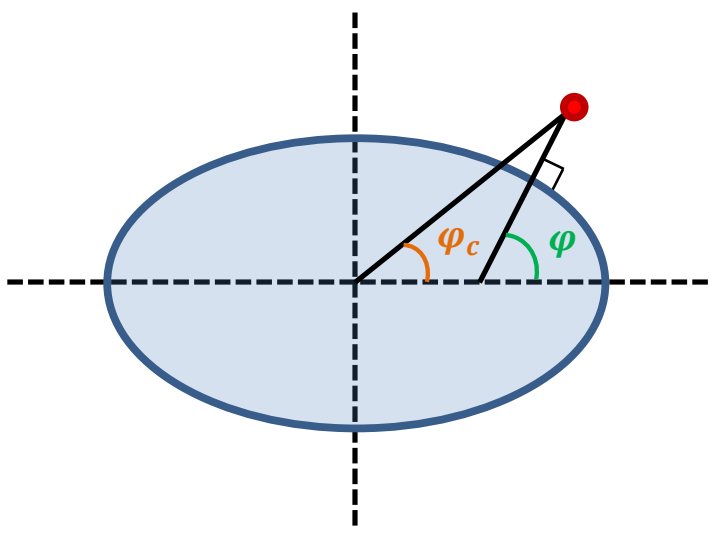

Figure 1-3: Geodetic versus Geocentric latitude measurements.

\subsubsection{Body Coordinate Frame}

The body coordinate frame is a non-inertial body-fixed frame, with the origin at a vehicle's center of mass. The x-axis points out through the nose of a vehicle. The y-axis points out of the right wing. The z-axis completes the right handed orthogonal system and points directly

down through the bottom of a vehicle ${ }^{36}$ as shown in Figure 1-4. The angular velocity defined in 
body coordinates, roll $(\mathrm{P})$, pitch $(\mathrm{Q})$, and $\operatorname{yaw}(\mathrm{R})$ are also defined in the positive direction ${ }^{36}$ in Figure 1-4. All static and dynamic stability and control terms are derived in this frame.

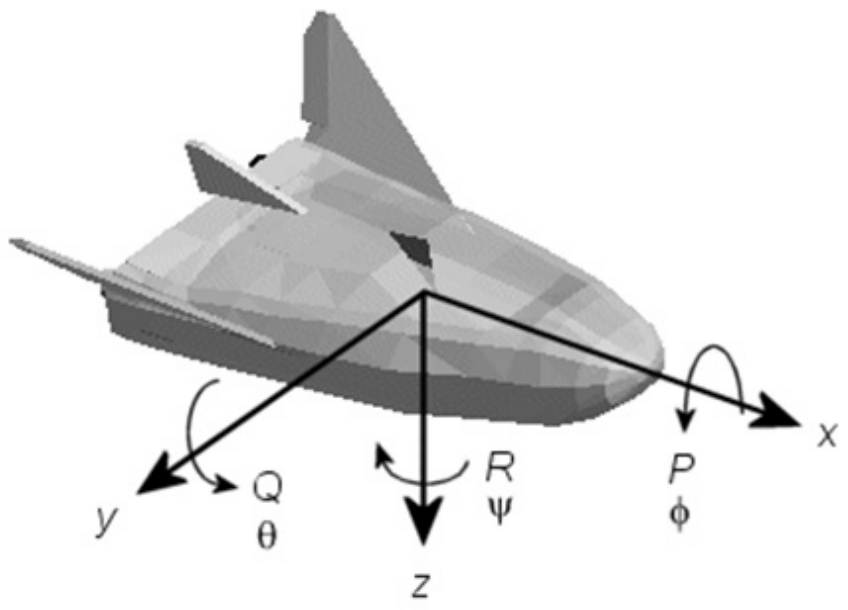

Figure 1-4: Body Coordinate Frame.

\subsubsection{Attitude Angles}

Both a position vector and attitude angles are needed to describe a vehicle's position in three dimensional space. Coordinate frames define a three dimensional space, but attitude angles describe an orientation within a three dimensional space. Quaternion and Euler angles define one reference frame with respect to another reference frame. Aerodynamic angles ( $\alpha$ and $\beta$ ), and flight path angles $(\gamma, \mu$, and $\chi)$ illustrate a vehicle's orientation within a frame.

\subsubsection{Quaternion}

Quaternion involves four parameters derived from an Euler axis, $e$, and a principal rotation angle, $\Phi$ to represent a unique orientation in space.

$$
\begin{aligned}
& q_{i}=e_{i} \sin \frac{\Phi}{2} \quad(i=1,2,3) \\
& q_{0}=\cos \frac{\Phi}{2}
\end{aligned}
$$


The first three parameters, $q_{1}, q_{2}$, and $q_{3}$, form a vector part of a quaternion describing a direction of a rotational axis. The scalar part, $q_{0}$, represents a rotation angle of that vector ${ }^{29}$. Quaternion parameters are used to calculate a direction cosine matrix (DCM). DCM represents a series of rotation to relate one coordinate frame orientation to another. DCM, as shown in Equation (1-2), represents an orientation of an arbitrary coordinate frame 2 with respect to an arbitrary coordinate frame 1 . A vector is transformed from frame 1 to frame 2 by pre-multiplying the $3 \times 3$ DCM in Equation (1-2) ${ }^{29}$ with a vector in frame 1.

$$
D C M_{2 / 1}=\left[\begin{array}{ccc}
q_{0}^{2}+q_{1}^{2}-q_{2}^{2}-q_{3}^{2} & 2\left(q_{1} q_{2}+q_{3} q_{0}\right) & 2\left(q_{1} q_{3}-q_{2} q_{0}\right) \\
2\left(q_{1} q_{2}-q_{3} q_{0}\right) & q_{0}^{2}-q_{1}^{2}+q_{2}^{2}-q_{3}^{2} & 2\left(q_{3} q_{2}+q_{1} q_{0}\right) \\
2\left(q_{1} q_{3}+q_{2} q_{0}\right) & 2\left(q_{2} q_{3}-q_{1} q_{0}\right) & q_{0}^{2}-q_{1}^{2}-q_{2}^{2}+q_{3}^{2}
\end{array}\right]
$$

The plant model propagates the body frame orientation in time using quaternion. Quaternion presents advantages over Euler angles for time-varying coordinate transformations since a singularity, introduced by Euler angles when the pitch angle is equivalent to $+/-90$ degrees, is eliminated ${ }^{29}$. However, a quaternion is hard to visualize so they have to be converted into Euler angles, which are easier to physically understand.

\subsubsection{Euler Angles}

Euler angles are a sequence of three right-handed angle rotations. Each rotation is represented by a DCM and multiplied together to create a DCM used to describe an orientation of a reference frame with respect to another much like a quaternion. Sequences of rotation and corresponding DCM are described below and depicted in Figure 1-5.

1. Rotation by an angle $\psi$ about the z-axis is called the yaw angle ${ }^{36}$. The DCM for the yaw angle rotation is described as the $3^{\text {rd }}$ matrix in Equation $(1-3)^{36}$. 
2. Rotation by an angle of $\theta$ about the $\mathrm{x}$-axis is called the pitch angle ${ }^{36}$. The DCM for the pitch angle rotation is described as the $2^{\text {nd }}$ matrix in Equation $(1-3)^{36}$.

3. The last rotation is about the $y$-axis given by an angle of $\phi$ is called the roll angle ${ }^{36}$. The DCM for the roll angle rotation is described as the $1^{\text {st }}$ matrix in Equation (1-3) ${ }^{36}$.

$$
\begin{aligned}
D C M_{2 / 1} & =\left[\begin{array}{ccc}
1 & 0 & 0 \\
0 & c \phi & s \phi \\
0 & -s \phi & c \phi
\end{array}\right]\left[\begin{array}{ccc}
c \theta & 0 & -s \theta \\
0 & 1 & 0 \\
s \theta & 0 & c \theta
\end{array}\right]\left[\begin{array}{ccc}
c \psi & s \psi & 0 \\
-s \psi & c \psi & 0 \\
0 & 0 & 1
\end{array}\right] \\
D C M_{2 / 1} & =\left[\begin{array}{ccc}
c \theta c \psi & c \theta s \psi & -s \theta \\
(-c \phi s \psi+s \phi s \theta c \psi) & (c \phi c \psi+s \phi s \theta s \psi) & s \phi c \theta \\
(s \phi s \psi+c \phi s \theta c \psi) & (-s \phi c \psi+c \phi s \theta s \psi) & c \phi c \theta
\end{array}\right]
\end{aligned}
$$

Where $c$ and $s$ are abbreviations for cosine and sine functions.

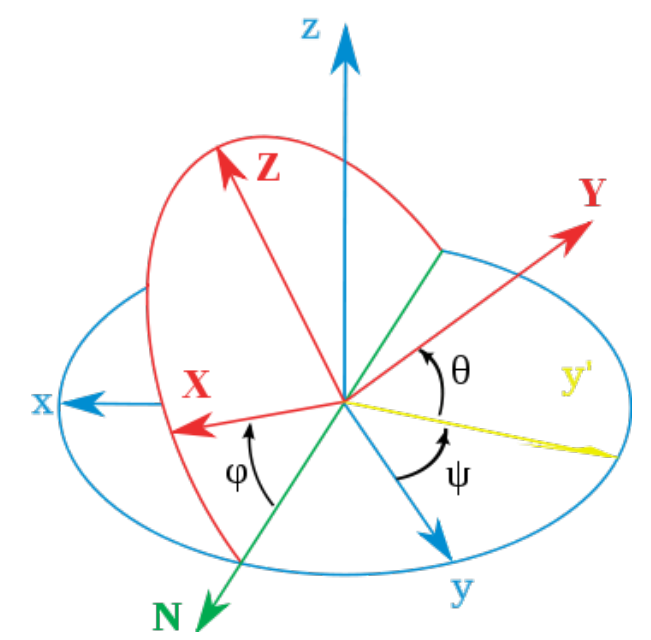

Figure 1-5: Euler angles representing an arbitrary coordinate transform.

Euler angles representing a transformation from coordinate frame 1 to coordinate frame 2 are computed using elements in a quaternion DCM from Equation $(1-2)^{29}$ :

$$
\begin{aligned}
& \theta=-\sin ^{-1}\left(c_{13}\right) \\
& \phi=\operatorname{atan} 2\left(c_{23}, c_{33}\right) \\
& \psi=\operatorname{atan} 2\left(c_{12}, c_{11}\right)
\end{aligned}
$$


Where the first subscript number indicates a row of a DCM and the second subscript indicates a column. Any coordinate transformation represented by a DCM could be used to resolve Euler angles for the same transformation using Equations (1-5) through (1-6).

\subsubsection{Aerodynamic Angles}

Forces and moments acting on the RLV are functions of aerodynamic angles and Mach number so it is necessary to compute these angles. Aerodynamic angles include angle of attack, $\alpha$, and sideslip angle, $\beta$. Velocity vector in body coordinates and vehicle body axes are used to resolve these angles. The angle between the $\mathrm{x}$-body axis and the velocity vector projected onto the vertical body axis plane, $x-z$ plane, is $\alpha$, computed in Equation $(1-8)^{36}$. The angle between the $\mathrm{x}$-body axis and the velocity vector projected onto the horizontal body plane, $x-y$ plane, is $\beta$ and is defined in Equation (1-9) ${ }^{36}$.

$$
\begin{gathered}
\alpha=\operatorname{atan} 2\left(w_{b}, u_{b}\right) \\
\beta=\operatorname{asin}\left(\frac{v_{b}}{\sqrt{u_{b}+v_{b}+w_{b}}}\right)
\end{gathered}
$$

Where $\mathrm{u}_{\mathrm{b}}, \mathrm{v}_{\mathrm{b}}, \mathrm{w}_{\mathrm{b}}$ are elements in the velocity vector relative to the body frame. Figure 1-6 shows positive displacements for both angles. 


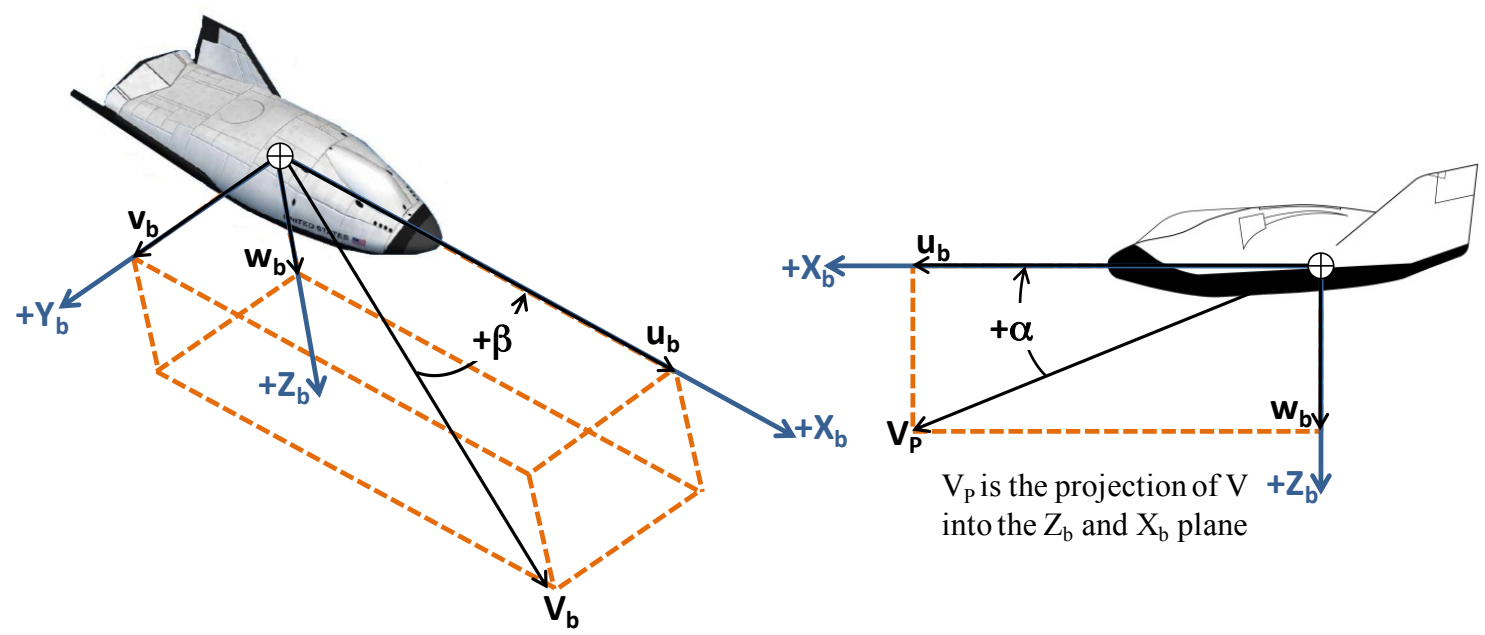

Figure 1-6: Definition of angle of attack and sideslip.

\subsubsection{Flight Path Angles}

Flight path angles are calculated using vectors in NED coordinates. Flight path angles are useful in analyzing a vehicle trajectory. Three angles in particular, $\gamma, \chi$, and $\mu$, define flight path direction. They are depicted in Figure 1-7. Vertical flight path angle is $\gamma$, which is a vertical angle between the local horizontal plane, the North-East plane, and the velocity vector projected on the North-Down plane ${ }^{29}$. Heading angle, $\chi$, is the angle between the North axis and the projection of the velocity vector on the horizontal North-East plane; positive is from North to East ${ }^{29}$. Bank angle is $\mu$, which is the angle between the vertical plane, formed by the vehicle velocity vector and the lift vector, and the vertical plane containing the velocity vector ${ }^{24}$. Bank angle describes the rotation about the velocity vector. The three angles are computed using Equations (1-10) through (1-12).

$$
\begin{array}{r}
\gamma=\operatorname{atan} 2\left(\frac{-V_{D}}{\sqrt{V_{N}^{2}+V_{E}^{2}}}\right) \\
\chi=\operatorname{atan} 2\left(\frac{V_{E}}{V_{N}}\right)
\end{array}
$$




$$
\mu=\operatorname{atan} 2\left(\frac{x_{1}}{x_{2}}\right)
$$

$$
\text { where } \quad \begin{aligned}
x_{1} & =\cos \alpha \cos \theta \cos \phi+\sin \alpha \sin \theta \\
x_{2} & =(\cos \theta \sin \phi) \cos \beta+(\cos \alpha \sin \theta-\sin \alpha \cos \theta \cos \phi) \sin \beta
\end{aligned}
$$

Where $\mathrm{V}_{\mathrm{N}}, \mathrm{V}_{\mathrm{E}}, \mathrm{V}_{\mathrm{D}}$ are elements of a velocity vector in NED coordinates, $\theta$ and $\phi$ are Euler angles describing an orientation of vehicle body coordinates with respect to NED coordinates.
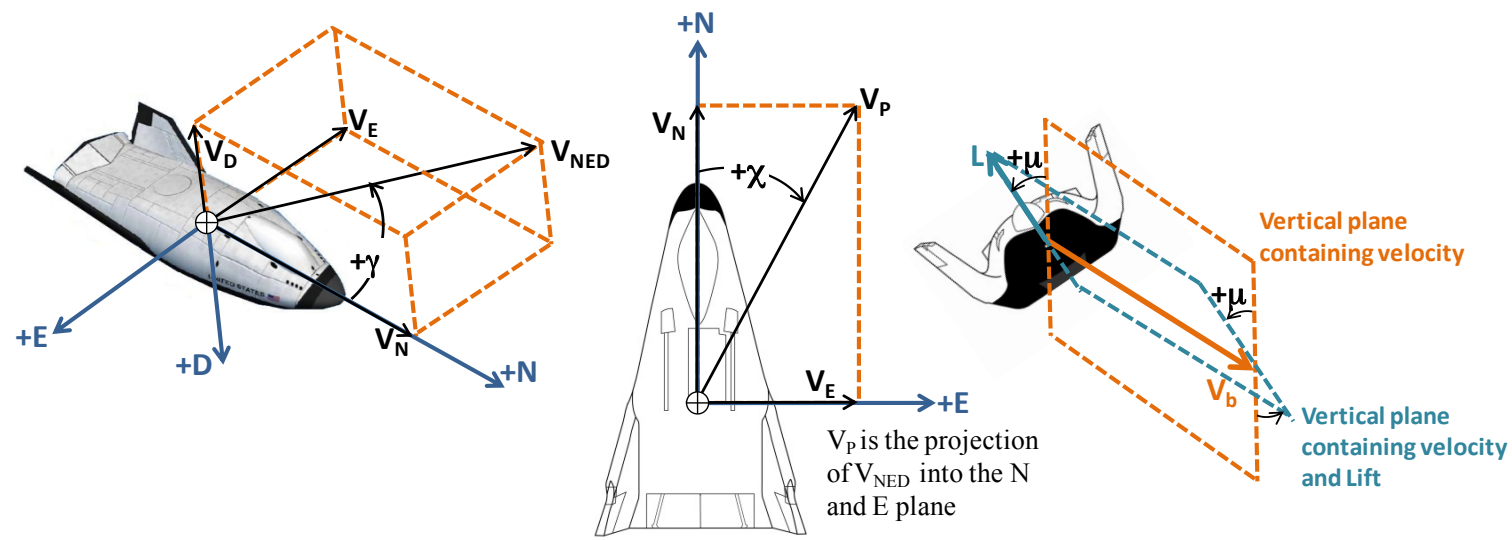

Figure 1-7: Definition of flight path angles.

Bank angle is important for maneuvering in the lateral direction. RLVs use bank to turn, rather than skid to turn, because any $\beta$ produces drag. Additional drag from $\beta$ bleeds off excessive amounts of energy. An RLV does not have a propulsive force to give the vehicle more energy in an unpowered approach and landing condition. An RLV only has a finite amount of energy so losing energy due to $\beta$ will cause the vehicle to deviate from its altitude profile, which cannot be recovered. Therefore, a controller must be designed to make an RLV do a coordinated turn in order to change direction. A coordinated turn changes a vehicle's direction by keeping the nose aligned with the velocity vector, meaning $\beta$ is equal to zero even if $\mu$ is nonzero. A controller will command an RLV to bank, causing the velocity vector to turn in a lateral direction to close the error on crosstrack. 


\subsubsection{Rotational and Kinematic Equation of Motion}

Rotational kinematics solves for the angular velocity vector, $\omega$, of a vehicle. The kinematic equation is applied to the body frame since the vehicle's moment is resolved in body coordinates. Angular velocity vector in the body frame includes the roll rate, P, pitch rate, Q, and yaw rate, R. Equation (1-13) ${ }^{29}$ solves for angular velocity in the body frame.

$$
\dot{\omega}=I^{-1}(M-\omega \times I \omega)
$$

$I$ is defined as an inertial matrix, and $M$ is a three element vector containing moments about the $\mathrm{x}$, $\mathrm{y}$, and $\mathrm{z}$ axis in body coordinates.

Angles used to define a coordinate transformation between a body axis and other reference frames change as the body frame moves in inertial space. The attitude kinematic equation below ${ }^{29}$ computes a quaternion rate, which is integrated to determine a quaternion describing a body frame orientation with respect to an inertial frame.

$$
\left[\begin{array}{l}
\dot{q_{0}} \\
\dot{q_{1}} \\
\dot{q_{2}} \\
\dot{q_{3}}
\end{array}\right]=\frac{1}{2}\left[\begin{array}{cccc}
0 & -P & -Q & -R \\
P & 0 & R & -Q \\
Q & -R & 0 & P \\
R & Q & P & 0
\end{array}\right]\left[\begin{array}{l}
q_{0} \\
q_{1} \\
q_{2} \\
q_{3}
\end{array}\right]
$$

Equation (1-2) is used to build a $\mathrm{DCM}_{\mathrm{ECI} / \mathrm{b}}$ from a quaternion. $\mathrm{DCM}_{\mathrm{b} / \mathrm{ECI}}$ will be used to transform forces from a body frame to an ECI frame to solve the translation equation of motion.

\subsubsection{Translational Dynamics}

Forces resolved in the body coordinates are transformed into ECI coordinates because Newton's Second Law is only valid in an inertial frame. Equation (1-2) could define a DCM $\mathrm{b}_{\mathrm{b} / \mathrm{ECI}}$ that takes vectors from ECI coordinates to body coordinates. $\mathrm{DCM}_{\mathrm{b} / \mathrm{ECI}}$ is transposed in order to 
reverse the transformation, from body coordinates to ECI coordinates, shown in Equation $(1-15)^{29}$. Equation (1-16), Newton's Second Law, is applied once forces are transformed in ECI coordinates.

$$
\begin{aligned}
F_{E C I} & =\left(D C M_{b / E C I}\right)^{T} F_{b} \\
F_{E C I} & =m a_{E C I}
\end{aligned}
$$

Where the $b$ subscript indicates a vector in body coordinates, and the $E C I$ subscript represents a vector in ECI coordinates, $F$ is a force vector, $m$ is the mass of a vehicle, and $a$ is an acceleration vector. The force from Equation (1-16) is divided by vehicle mass to calculate acceleration in the ECI frame. A series of integration from an acceleration vector computes position in the ECI frame.

\subsubsection{Coordinate Transformations}

Velocity and position vectors in inertial coordinates have to be further transformed to other reference frames mentioned in Section 1.5.1 using DCMs. Vehicle states needed to compute forces and moments, states used to define a vehicle's flight path and orientation, and states used in a backstepping controller can be calculated after vectors are transformed to other coordinates.

\subsubsection{ECI to Body Coordinates}

Transformation from ECI to body coordinates is done through a $\mathrm{DCM}_{\mathrm{b} / \mathrm{ECI}}$. $\mathrm{A} \mathrm{DCM}$ calculated using Equation (1-2) from a quaternion integrated from quaternion rates resolved by the kinematic equation of motion, Equation (1-14). $\alpha$ and $\beta$ are defined from a velocity vector in 
body coordinates relative to the vehicle. This relative velocity removes angular velocity from the Coriolis effect shown by Equation (1-17) ${ }^{29}$.

$$
\operatorname{Vrel}_{b}=D C M_{b / E C I}\left(V_{E C I}-\omega_{E} \times P_{E C I}\right)
$$

Where $\mathrm{Vrel}_{\mathrm{b}}$ is a relative velocity vector in body coordinate, ECI subscript signifies a vector in ECI coordinates, and $\omega_{\mathrm{E}}$ is the earth's rotation vector. $\alpha$ and $\beta$ are computed using Equation (1-8) and (1-9) with components from relative velocity. Magnitude of this velocity is used to derive Mach number and dynamic pressure in Equation (1-18) and (1-19):

$$
\begin{gathered}
\text { Mach }=\frac{\left\|\operatorname{Vrel}_{b}\right\|}{a} \\
Q b a r=\frac{1}{2} \rho\left\|\operatorname{Vrel}_{b}\right\|^{2}
\end{gathered}
$$

Where $a$ is speed of sound, Qbar is dynamic pressure, $\rho$ is density of ambient air.

\subsubsection{ECI to ECEF Coordinates}

ECEF coordinate frame is an intermediate frame used to obtain a navigational frame and a NED frame from an ECI frame. Vectors in a navigational frame and a NED frame are used to compute more useful vehicle states for feedback parameters in a backstepping controller, like $\mu$, $\gamma$, altitude, latitude, etc. ECEF coordinates rotates the $x-y$ plane with the earth. Thus, a vehicle's $\mathrm{x}-\mathrm{y}$ position in ECEF coordinates changes even if a vehicle's position does not in ECI coordinates $^{29}$. Greenwich mean sidereal time (GMST) describes the angular displacement of the ECEF $\mathrm{x}$-axis with respect to the ECI $\mathrm{x}$-axis, which is the vernal equinox, at time 0 Coordinated Universal Time $(\mathrm{UTC})^{25}$. Sidereal time defines an angular displacement of the ECEF x-axis at a particular mission elapsed time used to compute $\mathrm{DCM}_{\mathrm{ECEF} / \mathrm{ECI}}^{29}$. Sidereal time is computed in 
Equation (1-20), by summing the GMST at 0 UTC and the angular amount earth has rotated during an elapsed time from 0 UTC.

$$
\theta_{s}=\theta_{g}+\omega_{E} \Delta t
$$

Where $\theta_{\mathrm{s}}$ is sidereal time in radians, $\theta_{\mathrm{g}}$ is the GMST at $0 \mathrm{UTC}$ in radians, $\omega_{\mathrm{E}}$ is magnitude of earth's rotational rate in $\mathrm{rad} / \mathrm{sec}$, and $\Delta \mathrm{t}$ is elapsed time from $0 \mathrm{UTC}$ in seconds computed as:

$$
\Delta t=M E T+T_{0}
$$

Where $T_{0}$ is the amount of time that has passed in seconds since 0 UTC to mission start time, and MET is mission elapsed time in seconds. The plant model assumes mission start is at 0 UTC, then Equations (1-22) and (1-23) applies.

$$
\begin{gathered}
\Delta t=M E T+0 \\
\theta_{s}=\theta_{g}+\omega_{E} \mathrm{MET}
\end{gathered}
$$

The $\mathrm{DCM}_{\mathrm{ECEF} / \mathrm{ECI}}$ is an ECI $\mathrm{x}$-axis rotation about the z-axis by a sidereal time to align the ECI axes with the ECEF axes, which is represented by Equation $(1-24)^{29}$.

$$
D C M_{E C E F / E C I}=\left[\begin{array}{ccc}
\cos \theta_{s} & \sin \theta_{s} & 0 \\
-\sin \theta_{s} & \cos \theta_{s} & 0 \\
0 & 0 & 1
\end{array}\right]
$$

Position in ECEF coordinates, $\mathrm{P}_{\mathrm{ECEF}}$, can now be computed by:

$$
P_{E C E F}=D C M_{E C E F / E C I} P_{E C I}
$$

The Coriolis effect is added to an ECI velocity vector before a vector is transformed by a $\mathrm{DCM}_{\mathrm{ECEF} / \mathrm{ECI}}$ to form the total velocity vector in EFEC coordinates ${ }^{29}$, shown in Equation (1-26). 
Coriolis effect applies angular velocity experienced by the ECEF frame moving with respect to a stationary ECI frame.

$$
V_{E C E F}=D C M_{E C E F / E C I}\left(V_{E C I}+\omega_{E} \times P_{E C I}\right)
$$

Where $\mathrm{V}$ is a velocity vector, the $\mathrm{P}$ is a position vector, and $\omega_{\mathrm{E}}$ is earth's rotation rate in $\mathrm{rad} / \mathrm{sec}$ described as a vector:

$$
\omega_{E}=\left[\begin{array}{c}
0 \\
0 \\
7.2921 e^{-5}
\end{array}\right] \mathrm{rad} / \mathrm{sec}
$$

\subsubsection{ECEF to Geocentric and Geodetic Coordinates}

A position vector in Cartesian coordinates is projected onto an ellipsoid when transforming a position vector in ECEF coordinates to navigational coordinates: geodetic longitude, latitude, altitude, and geocentric latitude. An algorithm from Reference 6 was used to transform ECEF position vectors into geocentric and geodetic coordinates. The algorithm was proven to have minimal rounding errors over a wide variety of points in Cartesian space. The following equations show steps to compute geodetic latitude, longitude, altitude, and geocentric latitude ${ }^{6}$. 


$$
\begin{aligned}
r & =\sqrt{P_{x_{E C E F}}{ }^{2}+P_{y_{E C E F}}^{2}} \\
E & =\frac{b_{E} P_{z_{E C E F}}-\left(a_{E}{ }^{2}-b_{E}{ }^{2}\right)}{a_{E} r} \\
F & =\frac{b_{E} P_{z_{E C E F}}+\left(a_{E}{ }^{2}-b_{E}{ }^{2}\right)}{a_{E} r} \\
P & =\frac{4}{3}(E F+1) \\
Q & =2\left(E^{2}-F^{2}\right) \\
D & =P^{3}+Q^{2} \\
\text { if } D \geq & 0 \\
& v^{\prime}=\left(D^{1 / 2}-Q\right)^{1 / 3}-\left(D^{1 / 2}+Q\right)^{1 / 3} \\
\text { else } \quad & v=-\frac{\left(v^{\prime 3}+2 Q\right)}{3 P} \\
v= & 2 \sqrt{-P} \cos \left(\frac{1}{3} \cos ^{-1}\left(\frac{Q}{-P^{3 / 2}}\right)\right) \\
\text { end } & \\
G= & \frac{\left(E^{2}+v\right)^{1 / 2}+E}{2} \\
t= & \sqrt{G^{2}+\frac{(F-v G)}{2 G-E}-G}
\end{aligned}
$$

The $x$ and $y$ subscripts represent $x$ and $y$ elements in a position vector, and $a_{E}$ and $b_{E}$ are earth's semimajor and semiminor axes. Geodetic latitude, longitude, altitude, and geocentric latitude are calculated using Equation (1-28) through (1-31) once the above parameters are determined ${ }^{6}$.

$$
\begin{gathered}
\varphi=\tan ^{-1}\left(\frac{a_{E}\left(1-t^{2}\right)}{2 b_{E} t}\right) \\
\lambda=\tan ^{-1}\left(\frac{P_{y_{E C E F}}}{P_{x_{E C E F}}}\right) \\
h=\left(r-a_{E} t\right) \cos \varphi+\left(z-b_{E}\right) \sin \lambda \\
\varphi_{c}=\tan ^{-1}\left((1-f)^{2} \tan \varphi\right)
\end{gathered}
$$


Where $f$ is earth's flattening parameter, $\varphi$ is geodetic latitude, $\lambda$ is longitude, $\varphi_{c}$ is the geocentric latitude, and $h$ is the altitude.

$$
\begin{gathered}
D C M_{N E D / E C E F}=\left[\begin{array}{ccc}
c \varphi & 0 & s \varphi \\
0 & 1 & 0 \\
-s \varphi & 0 & c \varphi
\end{array}\right]\left[\begin{array}{ccc}
0 & 0 & 1 \\
0 & 1 & 0 \\
-1 & 0 & 0
\end{array}\right]\left[\begin{array}{ccc}
c \lambda & s \lambda & 0 \\
-s \lambda & c \lambda & 0 \\
0 & 0 & 1
\end{array}\right] \\
D C M_{N E D / E C E F}=\left[\begin{array}{ccc}
-s \varphi c \lambda & -s \varphi s \lambda & c \varphi \\
-s \lambda & c \lambda & 0 \\
-c \varphi c \lambda & -c \varphi s \lambda & -s \varphi
\end{array}\right]
\end{gathered}
$$

ECEF coordinates are rotated to NED coordinates by moving the ECEF $\mathrm{x}$-axis to a vehicle's longitude location first, represented by the third matrix in Equation (1-32) ${ }^{29}$. A left-handed 90degree rotation about the $y$-axis is performed to get the ECEF x-axis to align north and the z-axis to point down, shown as the second matrix in Equation $(1-32)^{29}$. Then, a left-handed rotation about the y-axis through a latitude angle aligns the ECEF coordinates with the NED coordinates, which is the first matrix in Equation (1-32) ${ }^{29}$. A position and velocity in NED coordinates are computed using the $\mathrm{DCM}_{\mathrm{NED} / \mathrm{ECEF}}$ :

$$
\begin{aligned}
& P_{N E D}=D C M_{N E D / E C E F} P_{E C E F} \\
& V_{N E D}=D C M_{N E D / E C E F} V_{E C E F}
\end{aligned}
$$

Velocity vector in NED coordinates from Equation (1-35) and Equations (1-10) and (1-11) are used to resolve flight path angles, $\gamma$ and $\chi$. Bank angle, $\mu$, computed from Equation (1-12) uses Euler angles, defining a transformation from NED coordinates to body coordinates, and aerodynamic angles. Euler angles from NED coordinates are computed from a $\mathrm{DCM}_{\mathrm{b} / \mathrm{NED}}$, which is a product of DCMs for various coordinates transformations already defined previously, as seen in Equation (1-36). 


$$
D C M_{b / N E D}=D C M_{b / E C I}\left(D C M_{E C E F / E C I}\right)^{T}\left(D C M_{N E D / E C E F}\right)^{T}
$$

Where $\mathrm{DCM}_{\mathrm{ECEF} / \mathrm{ECI}}$ is computed from Equation (1-24) and $\mathrm{DCM}_{\mathrm{NED} / \mathrm{ECEF}}$ is calculated from Equation (1-33). Elements in a $\mathrm{DCM}_{\mathrm{b} / \mathrm{NED}}$ coordinates are manipulated to resolve Euler angles from NED coordinates using Equations (1-37) to $(1-39)^{29}$.

$$
\begin{aligned}
& \theta=-\sin ^{-1}\left(\operatorname{DCM}_{b / N E D}(1,3)\right) \\
& \phi=\tan ^{-1}\left(\frac{\operatorname{DCM}_{b / N E D}(2,3)}{D C M_{b / N E D}(3,3)}\right) \\
& \psi=\tan ^{-1}\left(\frac{D C M_{b / N E D}(1,2)}{D C M_{b / N E D}(1,1)}\right)
\end{aligned}
$$

Section 1.5.5.1 through 1.5.5.3 concludes all of the coordinate transformation required through five coordinate frames to compute necessary vehicle states used for the RLV's guidance and control and the plant model used to simulate the RLV.

\subsubsection{Environment}

Atmosphere and gravity affects the magnitude of forces and moments acting on a vehicle so they need to be modeled accurately to simulate a vehicle's motion. The 1976 Committee on Extension to the Standard Atmosphere (COESA) was used to calculate density and speed of sound for a given altitude. Gravity is a mass attraction between two bodies. It is calculated from a gradient of a potential function. The WGS84 datum along with a potential function from the Earth Gravitation Model 1996 (EGM96) are used to calculate gravitational attraction. Table 1-1 defines the WGS84 parameter values and definitions used to describe earth's ellipsoid ${ }^{29}$. 
Table 1-1: WGS84 Ellipsoid Parameters

\begin{tabular}{|c|c|c|c|}
\hline Parameter & Name & Definition & Units \\
\hline $\boldsymbol{a}_{\boldsymbol{E}}$ & Semimajor axes & $6,378,137$ & $\mathrm{~m}$ \\
$\boldsymbol{b}_{\boldsymbol{E}}$ & Semiminor axes & $6,356,752$ & $\mathrm{~m}$ \\
$\boldsymbol{f}$ & Flattening & $=\frac{a-b}{a}$ & $\mathrm{~m}$ \\
$\boldsymbol{e}$ & Eccentricity & $=\frac{\left(a^{2}-b^{2}\right)^{1 / 2}}{a}$ & unitless \\
$\boldsymbol{\omega}_{\boldsymbol{E}}$ & Earth's rotation rate & $7.2921150 \times 10^{-5}$ & $\mathrm{rad} / \mathrm{s}$ \\
$\boldsymbol{G M}$ & Earth's Gravitation constant & $3986004.418 \times 10^{8}$ & $\mathrm{~m} / \mathrm{s}^{2}$ \\
\hline
\end{tabular}

The EGM96 model contains 130,676 coefficients for precise calculations, but only the first coefficient is used to calculate the potential function in Equation (1-40) ${ }^{29}$ since other coefficients are negligible in magnitude compared to the first coefficient.

$$
\begin{aligned}
& V=\frac{G M}{r}\left[1-0.5 J_{2}\left(a_{E} / r\right)^{2}\left(3 \sin ^{2} \varphi_{\mathrm{c}}-1\right)\right] \\
& \text { where } J_{2}=-\sqrt{5} C_{2,0}=1.0826267 \times 10^{-3}
\end{aligned}
$$

Where $r$ is the magnitude of $\mathrm{P}_{\mathrm{ECEF}}, \varphi_{\mathrm{c}}$ is the geocentric latitude, and $\mathrm{C}_{2,0}$ is the EGM96 coefficient.

Equation (1-41) ${ }^{29}$ calculates a gravitational attraction vector in ECEF coordinates, which takes the gradient of the potential function in geocentric coordinates from Equation (1-40) and transforms it into the ECEF frame.

$$
G^{e}=\frac{-G M}{r^{2}}\left[\begin{array}{l}
{\left[1+1.5 J_{2}(a / r)^{2}\left(1-5 \sin ^{2} \Psi\right)\right] p_{x} / r} \\
{\left[1+1.5 J_{2}(a / r)^{2}\left(1-5 \sin ^{2} \Psi\right)\right] p_{y} / r} \\
{\left[1+1.5 J_{2}(a / r)^{2}\left(3-5 \sin ^{2} \Psi\right)\right] p_{z} / r}
\end{array}\right]
$$

Where $G^{e}$ is the gravitational attraction vector in ECEF coordinates, and $p_{x}, p_{y}, p_{z}$ are elements of a position vector in ECEF coordinates. 
Forces produced from the centripetal acceleration at earth's surface have to be subtracted from the gravitational attraction vector in order to calculate gravity ${ }^{29}$.

$$
g_{E C E F}=G^{e}-\omega_{E} \times\left(\omega_{E} \times P_{E C E F}\right)
$$

Where $g_{E C E F}$ is the gravity vector in ECEF coordinates, $\omega_{E}$ is the angular rotation of the ECEF coordinate with respect to the inertial coordinate frame, equivalent to earth's rotational rate in vector form.

Gravitational forces are compiled in body coordinates before they are transformed into ECI coordinates to use Newton's Law to propagate a position vector. Therefore, a gravity vector must be in body coordinates in order to sum up gravitational forces and aerodynamic forces. A series of coordinate transformations are performed to calculate a gravity vector in body coordinates, as seen Equation (1-43).

$$
g_{b}=D C M_{b / E C I}\left(D C M_{E C E F / E C I}\right)^{T} g_{E C E F}
$$

Where $\mathrm{DCM}_{\mathrm{b} / \mathrm{ECI}}$ is defined by Equation (1-2) and $\mathrm{DCM} \mathrm{MCEF}_{\mathrm{ECI}}$ is defined by Equation (1-24).

\subsubsection{Reusable Launch Vehicle Parameters}

A sanitized set of aerodynamic data, geometry, and mass properties data from NGC's RLV configuration is used as the plant model mentioned previously in Section 1.4. The following sections describe how each set of data were provided. 


\subsubsection{Geometry and Mass Properties}

The scope of this thesis covers an approach and landing phase of an RLV's flight, which is unpowered. Therefore, mass properties are kept constant, assuming that fuel has been depleted. Table 1-2 and Table 1-3 show values used for the inertia matrix, mass, and geometric properties of the RLV for a vehicle simulation.

Table 1-2: Mass properties of the RLV

\begin{tabular}{|c|c|c|}
\hline Mass Properties & Value & Units \\
\hline mass & 6150 & slug \\
$\mathbf{I}_{\mathbf{x x}}$ & $1,110,400$ & slug- $\mathrm{ft}^{2}$ \\
$\mathbf{I}_{\mathbf{y y}}$ & $9,823,000$ & slug- $\mathrm{ft}^{2}$ \\
$\mathbf{I}_{\mathbf{z z}}$ & $10,400,000$ & slug- $\mathrm{ft}^{2}$ \\
$\mathbf{I}_{\mathbf{x y}}, \mathbf{I}_{\mathbf{y x}}$ & 0 & slug- $\mathrm{ft}^{2}$ \\
$\mathbf{I}_{\mathbf{x z}}, \mathbf{I}_{\mathbf{z x}}$ & 127,055 & slug- $\mathrm{ft}^{2}$ \\
$\mathbf{I}_{\mathbf{y z}}, \mathbf{I}_{\mathbf{z y}}$ & 0 & slug- $\mathrm{ft}^{2}$ \\
\hline
\end{tabular}

Table 1-3: Geometric properties of the RLV

\begin{tabular}{|l|l|l|}
\hline Geometric Properties & Value & Units \\
\hline Sref (reference area) & 3900 & $\mathrm{ft}^{2}$ \\
mac (mean aerodynamic chord) & 62 & $\mathrm{ft}$ \\
b (span) & 90 & $\mathrm{ft}$ \\
\hline
\end{tabular}

\subsubsection{Static Aerodynamics}

Static aerodynamic data are aerodynamic forces and moments generated from a bare airframe. All forces and moments are in coefficient form in the body frame as a nonlinear function of $\alpha$, Mach, and $\beta$ :

$$
[C A, C Y, C N, C l, C m, C n]=f c n(\alpha, M a c h, \beta)
$$


$C A$ is an axial force coefficient, which acts in an opposite direction of the body x-axis. $C Y, C N$ are coefficients of side force and normal force, respectively. Normal force acts in an opposite direction of the body z-axis. $\mathrm{Cl}, \mathrm{Cm}$, and $\mathrm{Cn}$ are moment coefficients about the x-axis, y-axis, and z-axis in body coordinates. The $\alpha$, Mach, and $\beta$ breakpoints are described below. Intermediate data points are linearly interpolated to compute forces and moment coefficients at flight conditions between breakpoints.

$$
\begin{gathered}
\alpha(\operatorname{deg})=[-10,-5,0,5,10,15,20,25,30,35,40,45,50,55,60,65] \\
M a c h=[1.2,1.5,2.0,3.0,4.0,5.0,6.0,8.0,10.0] \\
\beta(\operatorname{deg})=[-1,0,1]
\end{gathered}
$$

Equations (1-47) and (1-48) are used to compute the forces and moments from coefficient form.

$$
\begin{gathered}
{\left[\begin{array}{l}
X \\
Y \\
Z
\end{array}\right]=\frac{1}{2} \rho V^{2} \text { Sref }\left[\begin{array}{c}
-C A \\
C Y \\
-C N
\end{array}\right]} \\
{\left[\begin{array}{c}
L \\
M \\
N
\end{array}\right]=\frac{1}{2} \rho V^{2} \operatorname{Sref}\left[\begin{array}{c}
b(C l) \\
\operatorname{mac}(C m) \\
b(C n)
\end{array}\right]}
\end{gathered}
$$

Where $\rho$ is atmospheric density, $V$ is the mangitude of the relative velocity vector in body coordinates, Sref is the reference area.

\subsubsection{Dynamic Derivative Aerodynamics}

Dynamic derivative coefficients are changes in forces and moments due to angular velocity. These dynamic derivatives include damping terms, which oppose a vehicle's movement in order to dampen out its motion. Damping derivatives for the RLV are constant throughout the 
flight envelope. The dynamic derivatives are given in non-dimensionalized form so parameters in Table 1-4 are used to dimensionalize coefficients ${ }^{14}$ into moments.

Table 1-4: Dimensionalizing dynamic derivative terms

\begin{tabular}{|c|c|c|c|}
\hline $\begin{array}{c}\text { Dynamic } \\
\text { Derivative } \\
\text { Coefficient }\end{array}$ & Description & Value & $\begin{array}{l}\text { Dimensionalizing } \\
\text { Terms }\end{array}$ \\
\hline Clp & $\begin{array}{l}\text { Coefficient of roll, yaw, and side force due to } \\
\text { change in roll }\end{array}$ & -0.46 & $\frac{(\operatorname{span})(P)}{2(\text { Vtrue })}$ \\
\hline Cnr & $\begin{array}{l}\text { Coefficient of roll, yaw, and side force due to } \\
\text { change in yaw }\end{array}$ & -0.15 & $\frac{(\operatorname{span})(R)}{2(\text { Vtrue })}$ \\
\hline Cmq & $\begin{array}{l}\text { Coefficient of pitch, lift, and yaw due to } \\
\text { change in pitch }\end{array}$ & -0.68 & $\frac{(\operatorname{mac})(Q)}{2(\text { Vtrue })}$ \\
\hline
\end{tabular}

\subsubsection{Control Surface Aero}

The RLV has seven control surfaces used to maneuver and control the vehicle. The seven surfaces are defined as the flap, left rudder, right rudder, left inboard, right inboard, left outboard, and right outboard. The flap mainly produces a pitching moment and does not contribute to a yawing or rolling moment. The left and right rudders, the left and right inboards, and the left and right outboards contribute moments to all three axes. Each surface is deflected independently to provide the required control power. Changes in forces and moments due to control surface deflections are given as a function of $\alpha$, Mach, $\beta$, and surface deflection, $\delta$, shown in Equation (1-50). The $\alpha$, Mach, $\beta$ breakpoints from Equations (1-49) through (1-46) are the same for control surface aerodynamic data. Equations (1-52) and (1-51) show breakpoints for $\delta$.

$$
\begin{gathered}
{\left[\Delta C A_{i}, \Delta C Y_{i}, \Delta C Z_{i}, \Delta C l_{i}, \Delta C m_{i}, \Delta C n_{i}\right]=f c n\left(\alpha, \text { Mach, } \beta, \delta_{i}\right)} \\
\text { where } i=F, L R, R R, \text { Lin, Rin, Lout, Rout } \\
\delta_{F}(\operatorname{deg})=[-10,0,10,20,30] \\
\delta_{i}(\operatorname{deg})=[-30,-20,-10,0,10,20,30]
\end{gathered}
$$


Where the F subscript is for the flap, LR and RR subscript is for the left and right rudder, Lin and Rin subscript is for the left and right inboard, Lout and Rout is for the left and right outboard.

\subsubsection{Body Axis Transfer}

Aerodynamic data are given about a reference point in body coordinates in feet:

$$
\text { Aero Reference }=[-118,0,-7] f t
$$

However, forces and moments must be taken about a CG, shown in Equation (1-54) in order to propagate a vehicle's position properly.

$$
C G=[-117.5,0,-6.9] f t
$$

Moments are translated to a CG without additional moments to account for the translation. Translating forces to a CG location will produce moments, proportional to the moment arm to transfer a force to a new location. Equation (1-55) is the body-axis moment transfer theorem used to compute additional moments from moving forces from an aerodynamic reference point to a CG.

$$
\begin{gathered}
{\left[C_{l_{C G}}, C_{m_{C G}}, C_{n_{C G}}\right]=\left[C_{l_{r e f}}, C_{m_{r e f}}, C_{n_{r e f}}\right]+\left(\left[x_{r e f}, y_{r e f}, z_{r e f}\right]-\left[x_{C G}, y_{C G}, z_{C G}\right]\right)} \\
\times\left[C_{X_{r e f}}, C_{Y_{r e f}}, C_{Z_{r e f}}\right] . /[\text { span }, \text { mac }, \text { span }]
\end{gathered}
$$

Where $x_{r e f}, y_{r e f}, z_{r e f}$, represents an aerodynamic reference location; $C l_{r e f}, C m_{r e f}, C n_{r e f}$ are moment coefficients of roll, pitch, yaw defined from an aerodynamic reference; $C X_{r e f}, C Y_{r e f}, C Z_{r e f}$ are force coefficients in the X-direction, Y-direction, and Z-direction in body coordinates defined from an aerodynamic reference; Span and mac are the RLV's span and mean aerodynamic chord in feet. 


\subsection{Implementation of the Plant Model into Simulink}

A system architecture is designed to encapsulate various functionalities needed to simulate the RLV motion with the controller. The system architecture will be introduced in this section, but each subsystem implementation will be explained as they are developed throughout the paper. This section will focus mainly on implementing the plant model from the equations formulated in the previous section, 1.5. Several key Simulink tools are used to facilitate organization and development of each subsystem in the architecture. These tools include bus objects and model references explained in subsequent sections, 1.6.1 and 1.6.2.

\subsubsection{Bus Objects}

Bus objects are useful in explicitly controlling a system interface. A signal name, data type, and dimension have to be specified for each bus object element. This guarantees that right elements are passed into inputs and outputs of a system, which reduce instances of passing wrong signals within a modeling environment. Simulink models are more "readable" when bus objects are passed throughout a model since it compiles all output signals into one. Bus object compilation prevents signals from crossing, which may create a web of signals where their origin may be hard to decipher. To keep track of one signal is less complicating than to trace multiple signals passed between subsystems. Moreover, bus selectors allow a user to easily select desired signals needed for certain subsystems. Bus objects are defined for inports and outports for each system in the architecture. Figure 1-8 illustrates benefits of using bus objects with an arbitrary model. 


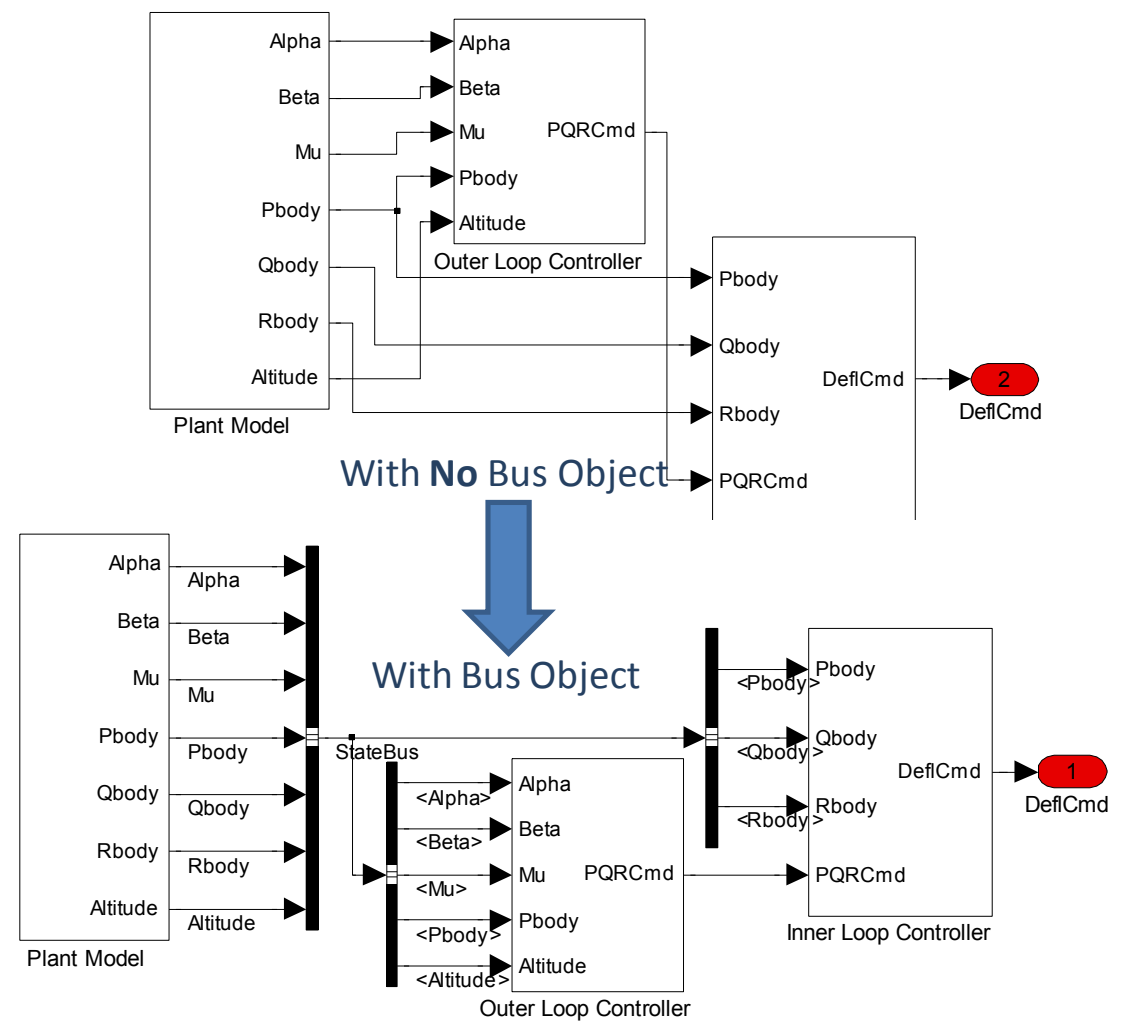

Figure 1-8: Modeling with and without a bus object in Simulink.

\subsubsection{Model References}

Model references are used throughout the Simulink model to represent major subsystems. Model references are built to stand alone so component testing is easy. Turning a subsystem into a model reference is also advantageous when a subsystem block contains many computationally intensive blocks like pre-look up and look up tables. Simulink Real Time Workshop writes and compiles C-code for a subsystem contained in a model reference called a MEX-file, which stands for Matlab executable file. Model execution speeds up when model references are in accelerated mode because Simulink will use this compiled code to run model references ${ }^{42}$. Otherwise, Simulink will execute a model reference interpretively like any other subsystem in a top-level model if normal mode is chosen, which is slower than running an executable ${ }^{42}$. The backstepping controller, dynamic inversion controller, and the plant aerodynamic model blocks were turned 
into model references because each of these blocks contained many look-up tables that slowed down the simulation time. Figure 1-9 depicts an execution of an example model reference when a Simulink model is initiated.

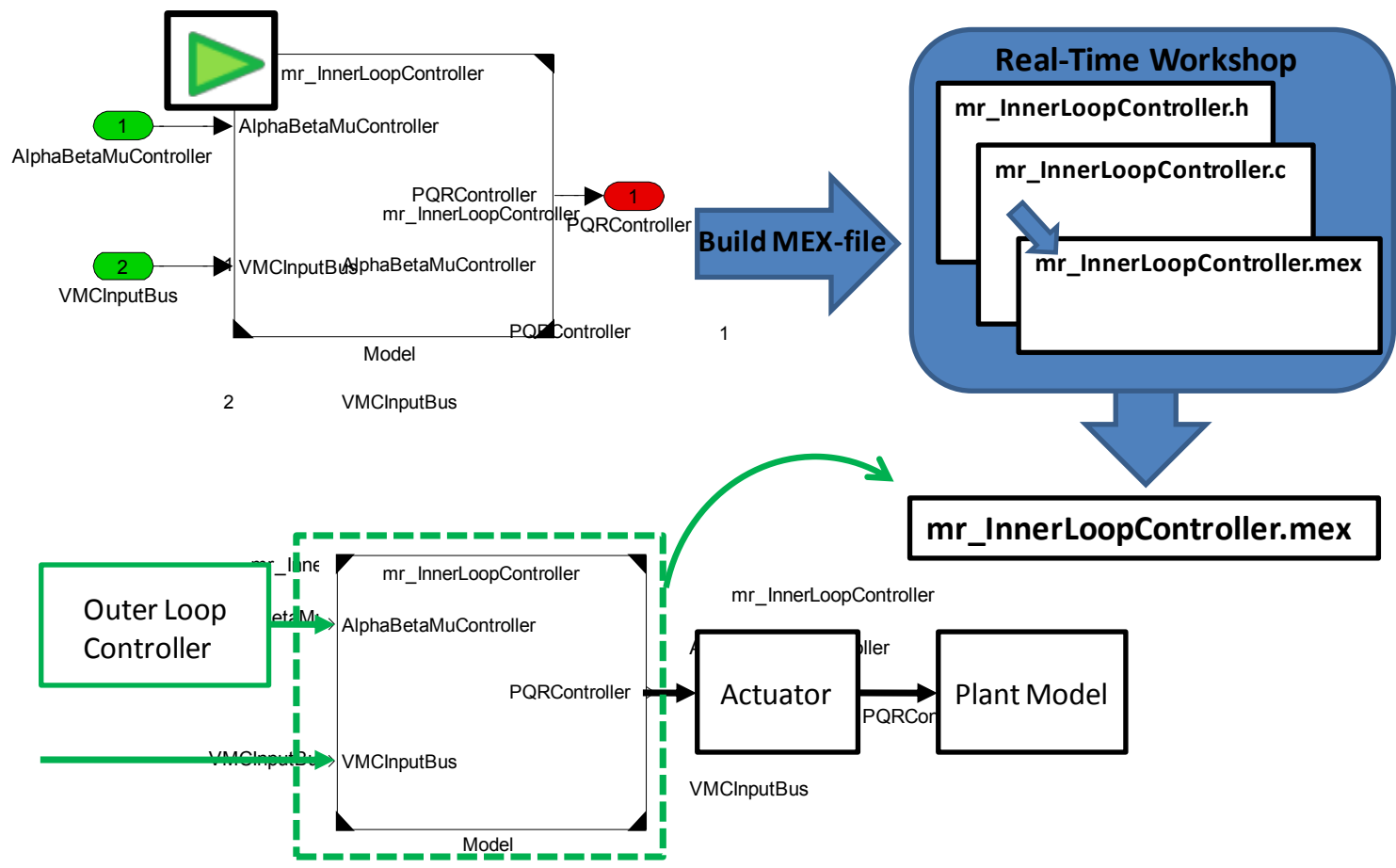

Figure 1-9: Model Reference compiling and running in Simulink.

Real Time Workshop creates a C-code from all block functions inside a model reference and then compiles a C-code into a MEX-file. This MEX-file is called every time Simulink calls the model reference when the simulation is run.

\subsubsection{System Architecture}

The system architecture is designed as seen in Figure 1-10. Three major subsystems exist at the top level of the Simulink model: Vehicle Management Computer (VMC), Actuator, and the Plant. Each of these subsystems are created into a model reference. Bus objects are formed to pass signals between each model reference. The VMC is the only subsystem that is installed on 
the RLV. The Actuators are a physical part of the RLV. The Plant subsystem contains functions to simulation the RLV and the environment, and is not contained on the actual vehicle. The system architecture is missing a sensor and navigation subsystem that should be at the output of the plant. However, the scope of this thesis covers uncertainties in aerodynamic terms and assumes vehicle states are exactly measured and known. Therefore, these states are fed directly through from the Plant subsystem output to the VMC input. A full vehicle subsystem simulation will contain a sensor and navigation subsystems which output sensed states and navigational data from instruments on a vehicle. This data will be inputs to the VMC, which will include algorithms to filter out noise and couple various sensed data to compute vehicle states used for the controller. 


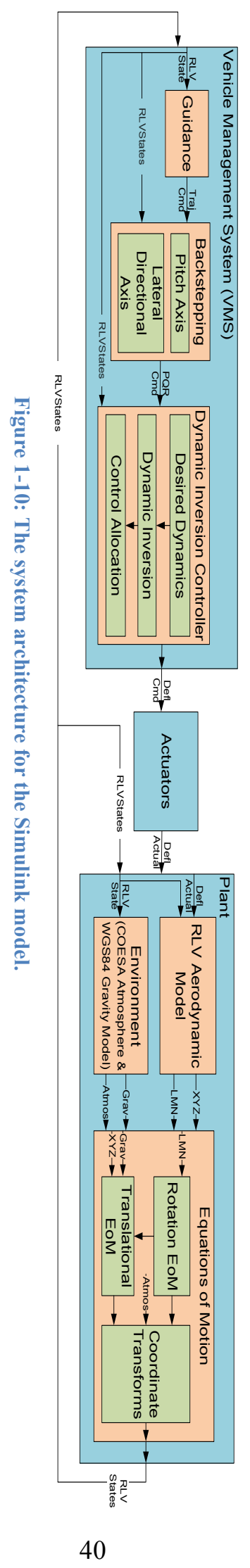




\subsubsection{Vehicle Management Computer (VMC)}

The Vehicle Management Computer (VMC) is a processor inside a vehicle that performs all of the core computation to generate trajectory commands, guidance commands from the backstepping controller, and control deflection commands from the dynamic inversion controller. Within the VMC block are four subsystems: VMC Translator, Trajectory Command, Backstepping Controller, and Dynamic Inversion Controller. The VMC translator takes the "StateBus", which is a bus object of outputs from the Plant, and the "ActuatorBus", which is a bus object of actuator signals, as inputs to select only necessary signals for the trajectory, backstepping, and dynamic inversion controller subsystem blocks. Detailed models of the trajectory block and the controller blocks, containing backstepping and dynamic inversion, are described in future sections after formulations are completed.

\subsubsection{Actuator Model}

Actuators are created as linear models with only a rate limit and a maximum and minimum deflection limit imposed, as seen in Table 1-5. Therefore, a commanded actuator deflection from the controller will equal the actual deflection unless an actuator deflection rate or position limits are exceeded by the controller commands. Rate limiter blocks are used to enforce actuator limits and saturation blocks are used to enforce deflection limits inside the Simulink model shown in Figure 1-11. 
Table 1-5: Control Deflection Maximum and Minimum Limits.

\begin{tabular}{|l|c|c|c|}
\hline \multicolumn{1}{|c|}{ Actuator Type } & $\begin{array}{c}\text { Rate limits } \\
\text { (deg/sec) }\end{array}$ & $\begin{array}{c}\text { Minimum } \\
\text { Deflection (deg) }\end{array}$ & $\begin{array}{c}\text { Maximum } \\
\text { Deflection (deg) }\end{array}$ \\
\hline Flap & $+/-60$ & -10 & 30 \\
Left Rudder & $+/-60$ & -30 & 30 \\
Right Rudder & $+/-60$ & -30 & 30 \\
Left Inboard & $+/-60$ & -30 & 30 \\
Right Inboard & $+/-60$ & -30 & 30 \\
Left Outboard & $+/-60$ & -30 & 30 \\
Right Outboard & $+/-60$ & -30 & 30 \\
\hline
\end{tabular}

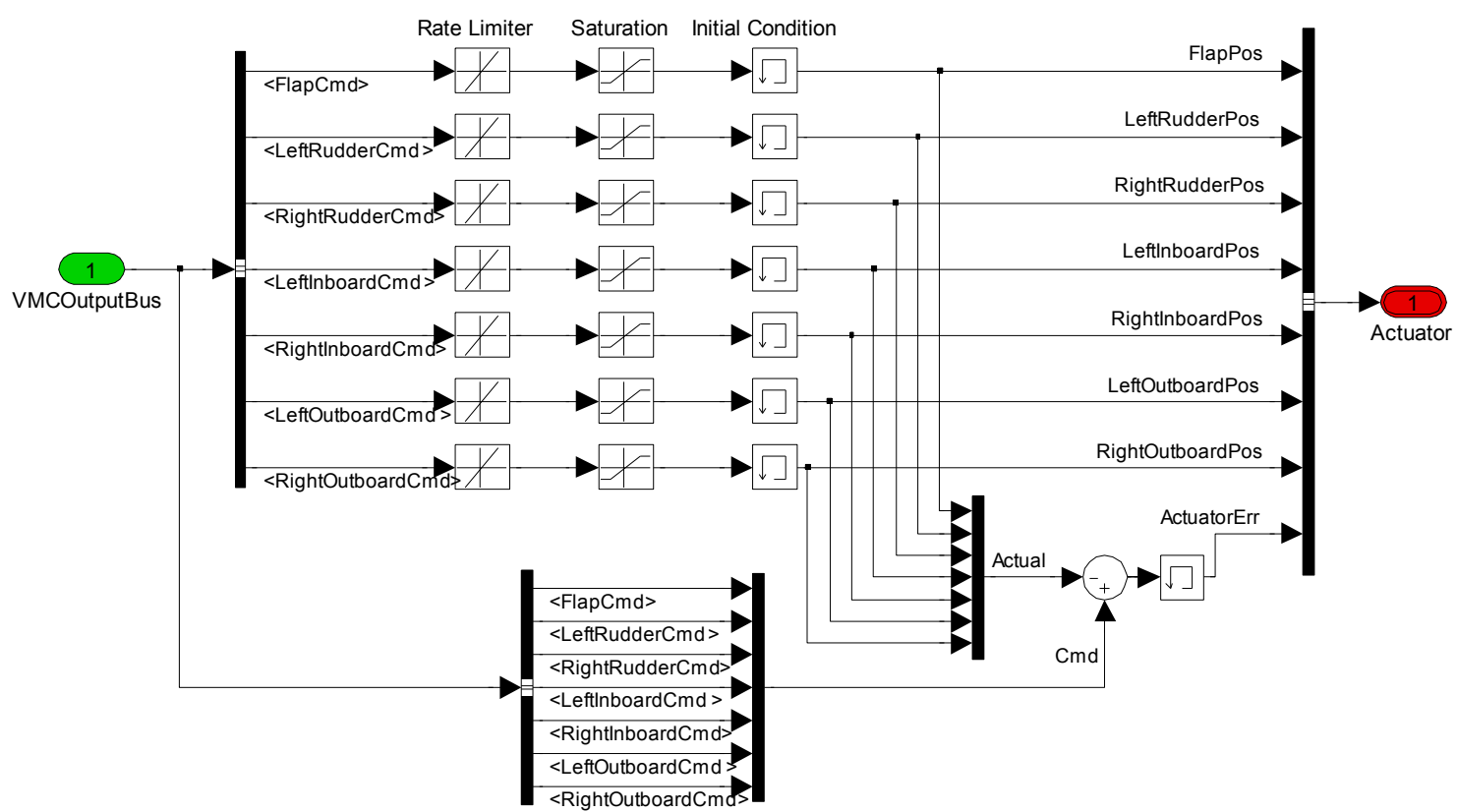

Figure 1-11: Actuator model inside Simulink.

An algebraic loop is a problem encountered from modeling a physical system. It is caused by a feedback loop created from the Plant output feeding into the controller. Simulink does not know where to start the simulation because the Plant needs to know control surface deflections to calculate forces and moments to compute vehicle states, but the controller needs vehicle states to compute control surface deflections. Memory blocks are used to specify initial conditions for each control surface to break the algebraic loop in the Simulink model. Memory blocks will also allow a user to initialize specific trimmed control surface deflections. Test cases 
for robustness analysis calls for the RLV to initialize at a flying flight condition, so the vehicle will experience transients when it is not initialized in a trimmed condition.

\subsubsection{Plant}

The Plant block contains all of the subsystem required to simulate a vehicle motion in three dimensional space. These subsystems include the "RLV Aerodynamics", the "Environment", and the "Equations of Motion" block depicted in Figure 1-12.

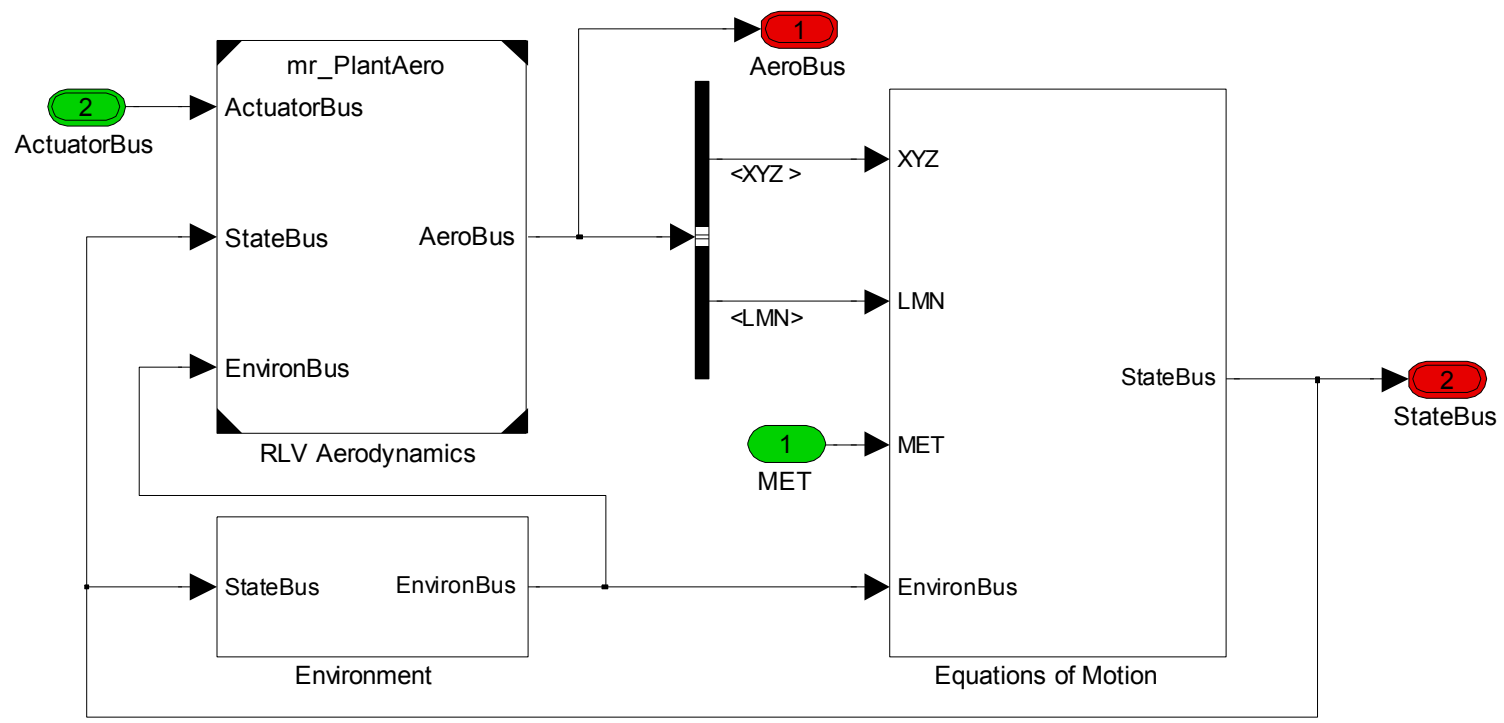

Figure 1-12: The Plant Model implementation into Simulink.

\subsubsection{RLV Aerodynamics}

The RLV aerodynamic subsystem is further broken up into two subsystems. One compiles all forces and moments from a bare airframe, which includes static aerodynamic data and dynamic aerodynamic data. The other subsystem adds all forces and moments contributing from control surfaces, shown in Figure 1-13. Gravitational forces in body coordinates are summed with aerodynamic forces to produce total body forces acting on a vehicle. 


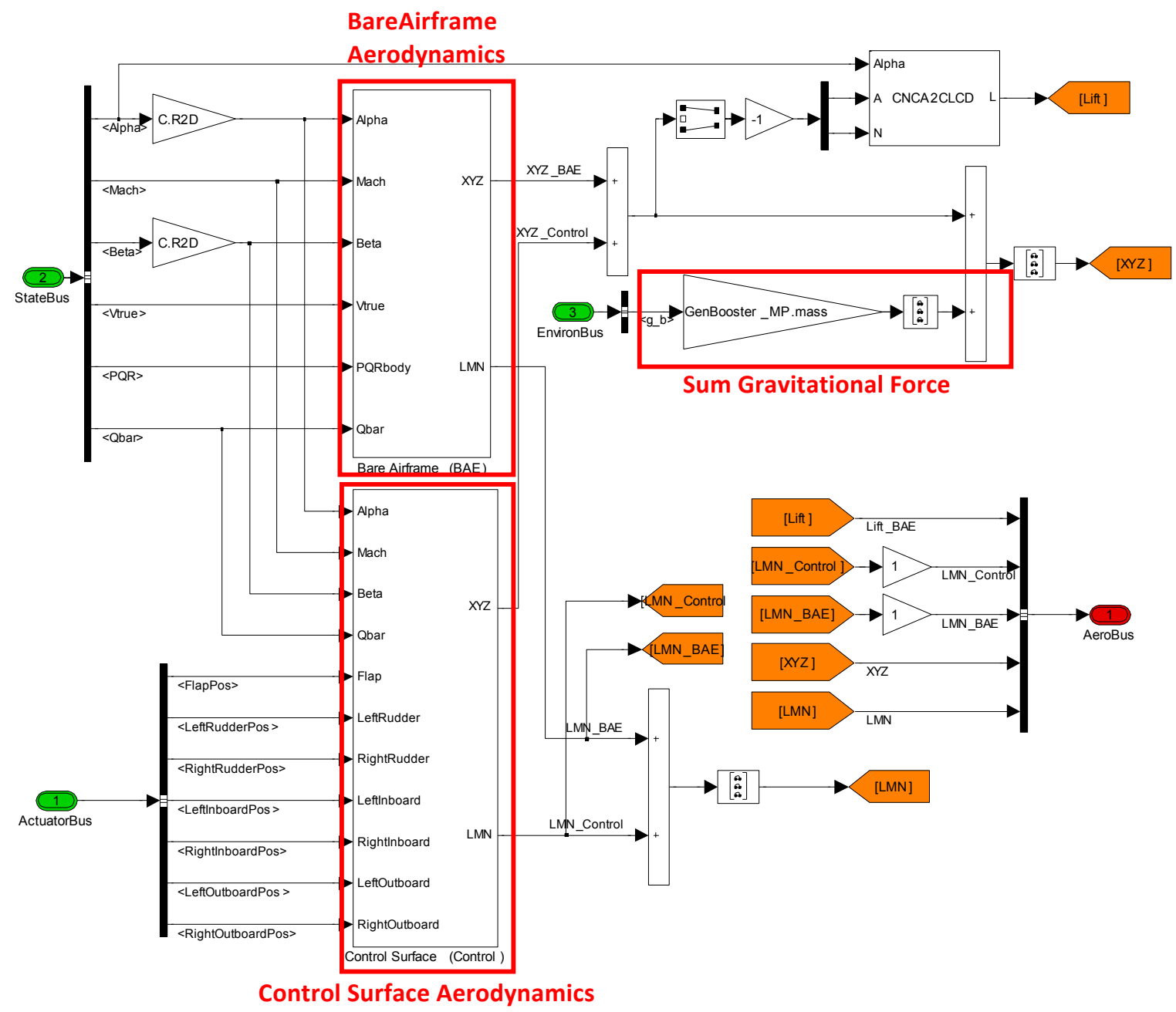

Figure 1-13: Inside the Plant Aerodynamics Block.

Within each bare airframe aerodynamics and control surface aerodynamics subsystem block, a pre-look and look up table is created for each aerodynamic coefficient term from Equations (1-44) and (1-50). These tables linearly interpolate aerodynamic terms at flight conditions between break points. Similar aerodynamic coefficient terms are summed up. Then, forces and moments are transferred to the CG using the body-axis moment transfer from Equation (1-55). Force transfers results in additional moments on a vehicle caused by the moment arm from the aerodynamic reference point to the CG, which are summed with existing moments acting on the vehicle. Aerodynamic coefficient terms are turned into forces and moments, which becomes inputs to the rotational and translational equations of motion. Figure 1-14 depicts the 
process above for the bare airframe subsystem from Figure 1-13. Forces and moments contributing from the control surface aerodynamics are modeled similarly to the bare airframe. The only differences are the data for the table look up blocks used to compute coefficients before they are all summed up.

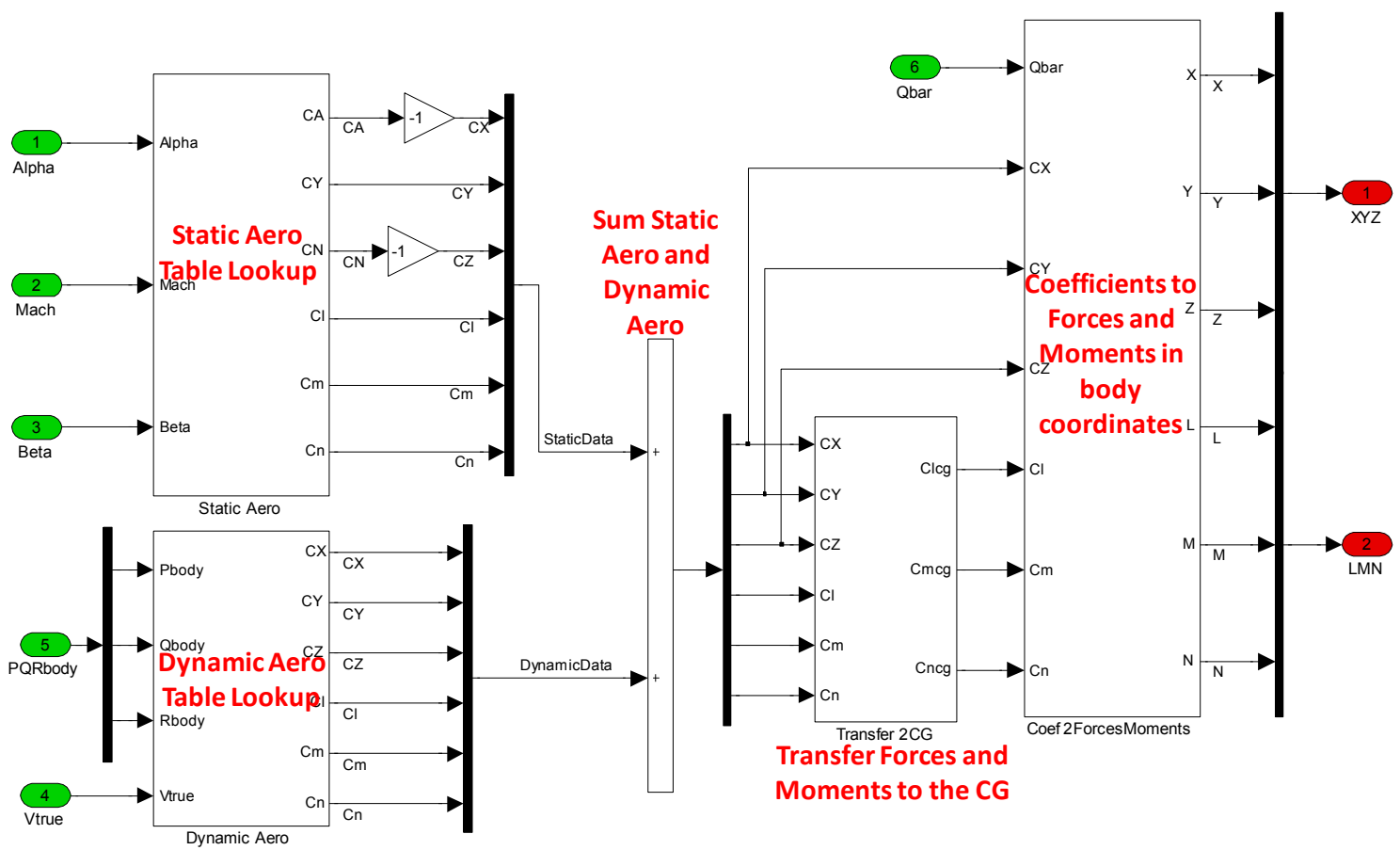

Figure 1-14: Simulink implementation of forces and moments computation from the bare airframe.

\subsubsection{Environment}

The Environment block contains the COESA atmosphere block and the gravity embedded matlab (EML) block seen in Figure 1-15. The COESA atmosphere block is from the Aerospace Toolbox used in order to calculate density and speed of sound from a vehicle's current altitude. The EML block contains Equations (1-42) and (1-43) to calculate gravity in ECEF coordinates. Gravity in ECEF coordinate is transformed into body coordinates using a DCM so gravity forces can be summed with aerodynamic forces. Memory blocks are used to initialize 
vehicle states to break an algebraic loop. This loop is formed because gravity forces and atmospheric density is needed to calculate the total force to propagate vehicle position, but gravity and atmospheric models need vehicle position.

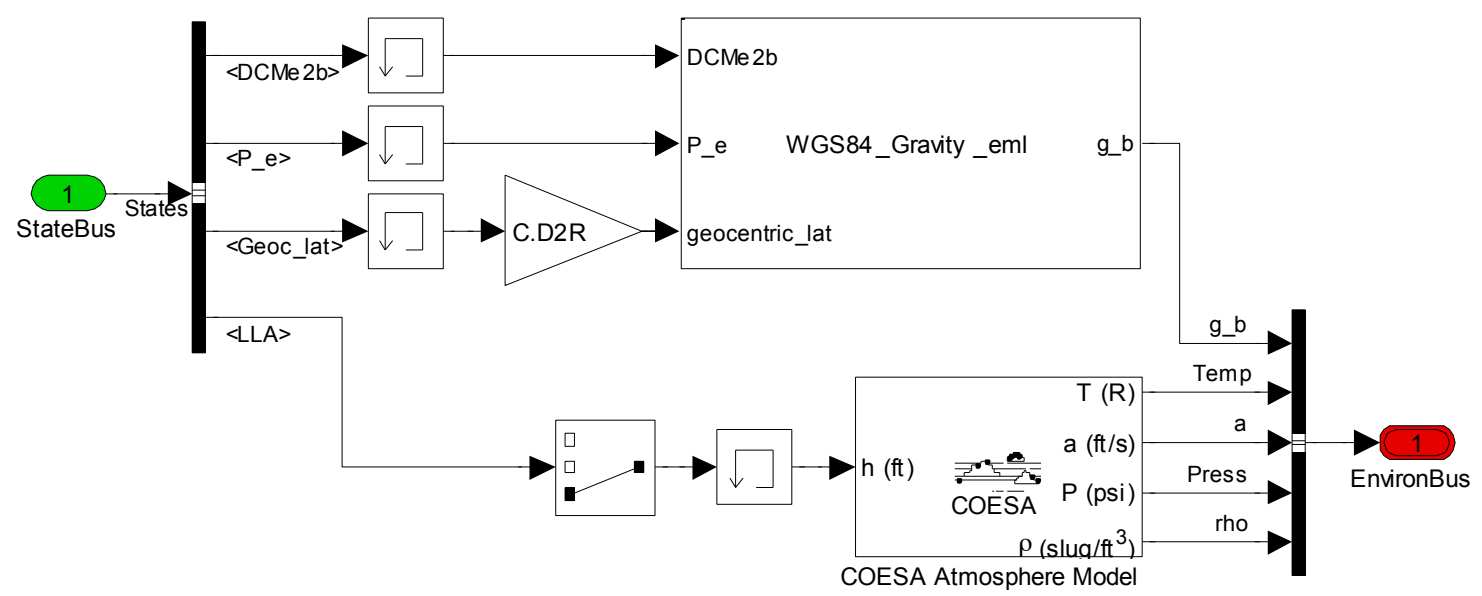

Figure 1-15: Inside the Environment Block in Simulink.

\subsubsection{Equations of Motion}

The Equations of Motion block contains three subsystems shown in Figure 1-16. The rotational kinematics from Equations (1-13) and (1-14) are modeled inside "Rotational EoM" block, which computes angular body rates and a DCM describing an orientation of the body frame with respect to the inertial frame, seen in Figure 1-17. The DCM along with the body forces, are fed into the "Translation EoM" block to calculate acceleration, velocity, and position of the RLV in ECI coordinates from Equation (1-16), depicted in Figure 1-17. The coordinate transformation block takes acceleration, velocity, position vectors, and Euler angles resolved in ECI coordinates to compute vectors and angles used to analyze vehicle maneuvers along a flight path. The coordinate transformation block contains all of the computation from Section 1.5.5. 


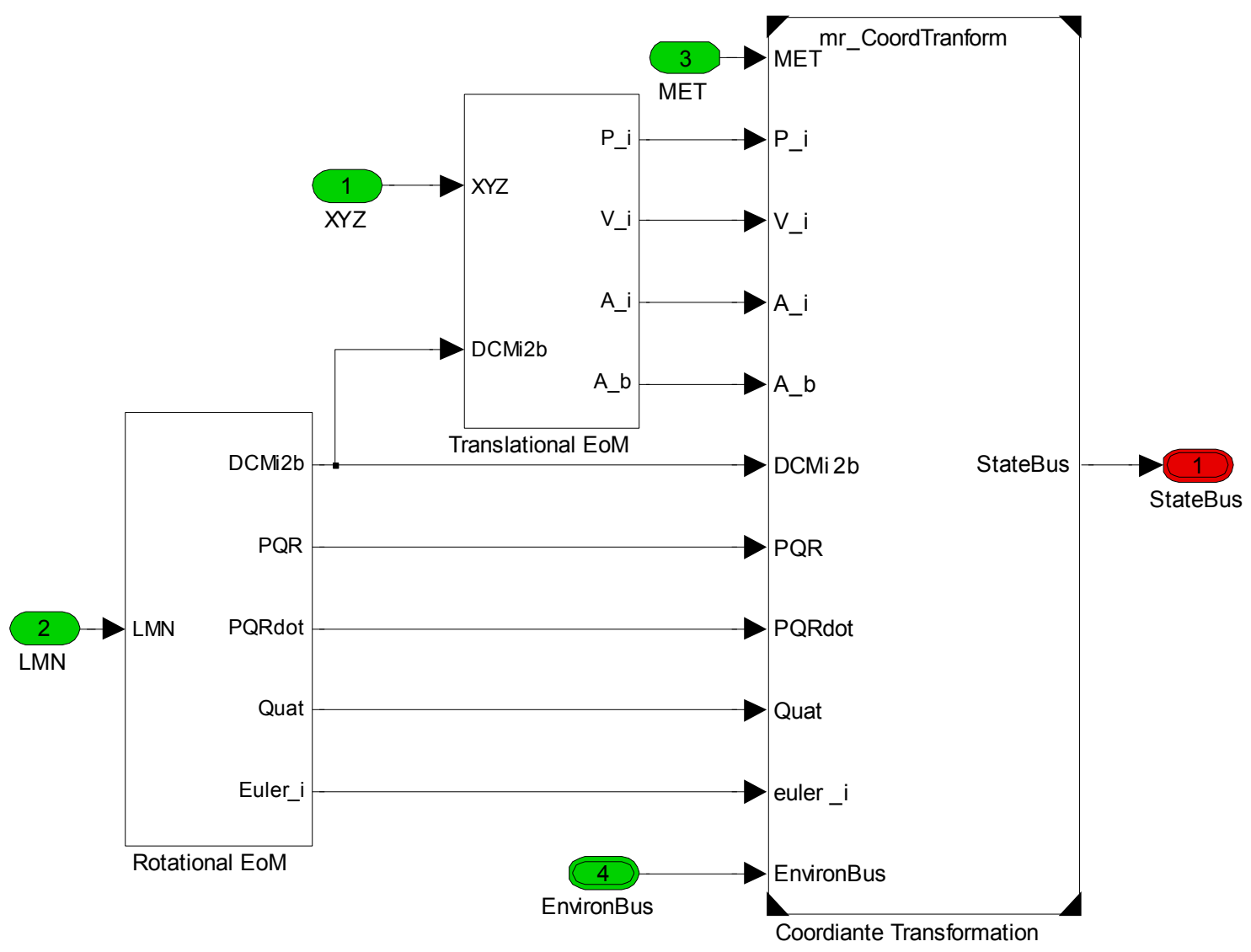

Figure 1-16: Simulink implementation of the equations of motion. 


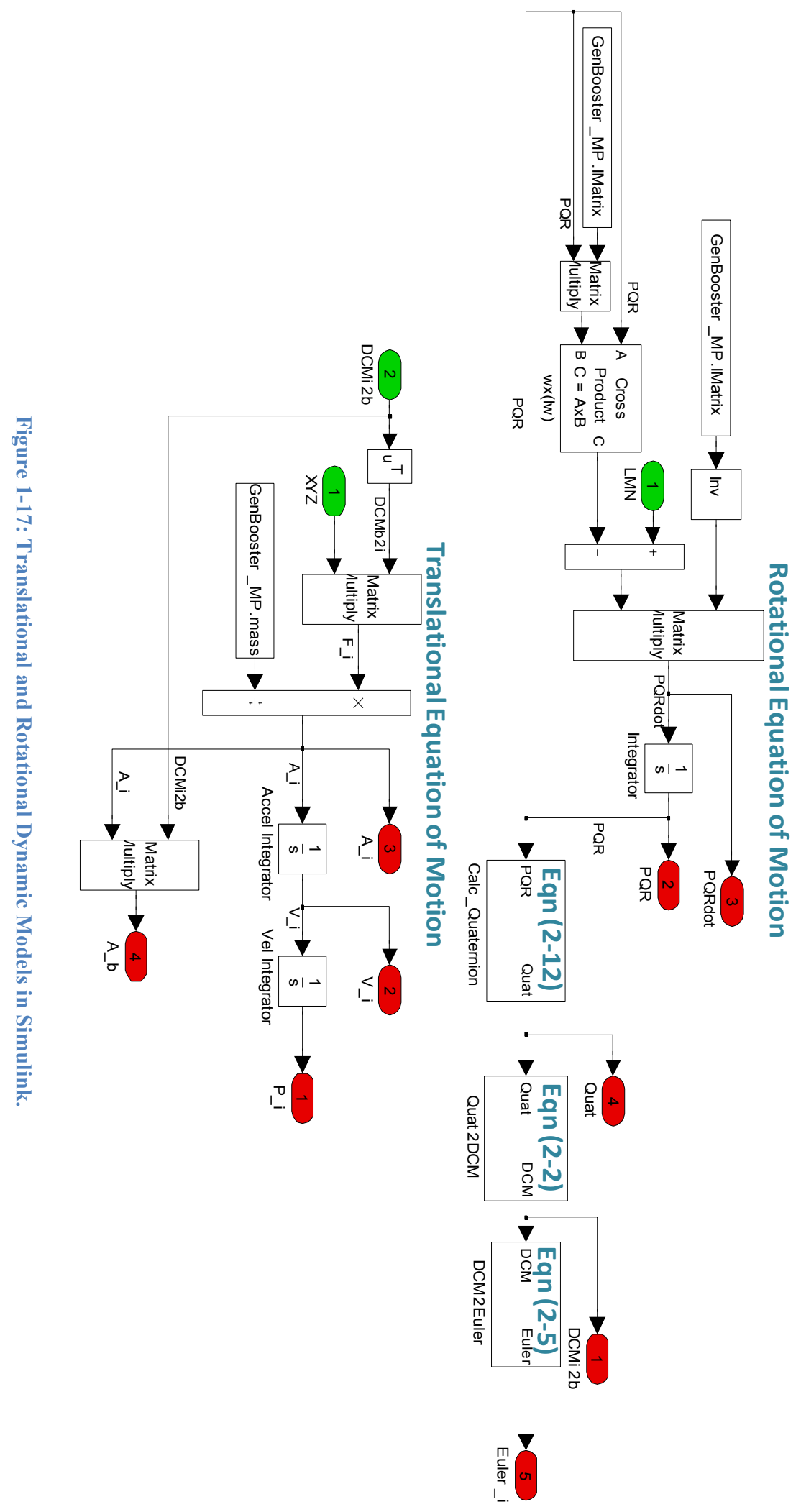




\subsection{Reference Trajectory}

\subsubsection{Motivation}

Initially, a $\gamma$ command schedule was created based on the RLV's current altitude. The command schedule included two phases like the space shuttle: a steep glideslope and a final flare. As a vehicle descends with a constant steep glideslope command, atmospheric density increases causing dynamic pressure to increase even if $\alpha$ and velocity are constant. Increase in dynamic pressure will cause a vehicle to produce more lift and drag, exciting the phugoid mode. The phugoid mode causes oscillations in altitude by an exchange of potential and kinetic energy. The increase in lift and drag will cause kinetic energy to decrease, causing an increase in potential energy observed by a rise in altitude. $\alpha$ decreases to close altitude error causing a decreased lift and drag, resulting in velocity increase and altitude drop from a rise in kinetic energy and a fall in potential energy. The altitude oscillation causes energy to bleed off, decreasing the maximum range a vehicle can attain. Moreover, the exact flight path angle command needed to achieve a desired touchdown point was hard to determine through a trial and error procedure. Thus, Reference 27 is used to compute a feasible approach and landing profile to glide the RLV down to a desired touchdown condition.

\subsubsection{Formulation}

The approach and landing algorithm uses downrange as an independent variable to integrate approximations of the governing equations for three phases of landing. The three phases: steep glideslope, circular pull-up, and flare, are shown in Figure 1-18. The trajectory is iterated upon one free-tuning parameter until these phases can be integrated backward from 
touchdown to the approach and landing interface (ALI) condition while maintaining continuity between each phase ${ }^{27}$. The governing equations used in the trajectory generation assume $\beta$ and $\mu$ are equal to zero degrees. This assumption is valid since the RLV should be lined up with runway heading at the end of the Terminal Area Energy Management (TAEM) phase, so minimal banking will be required to keep the RLV aligned with a runway.

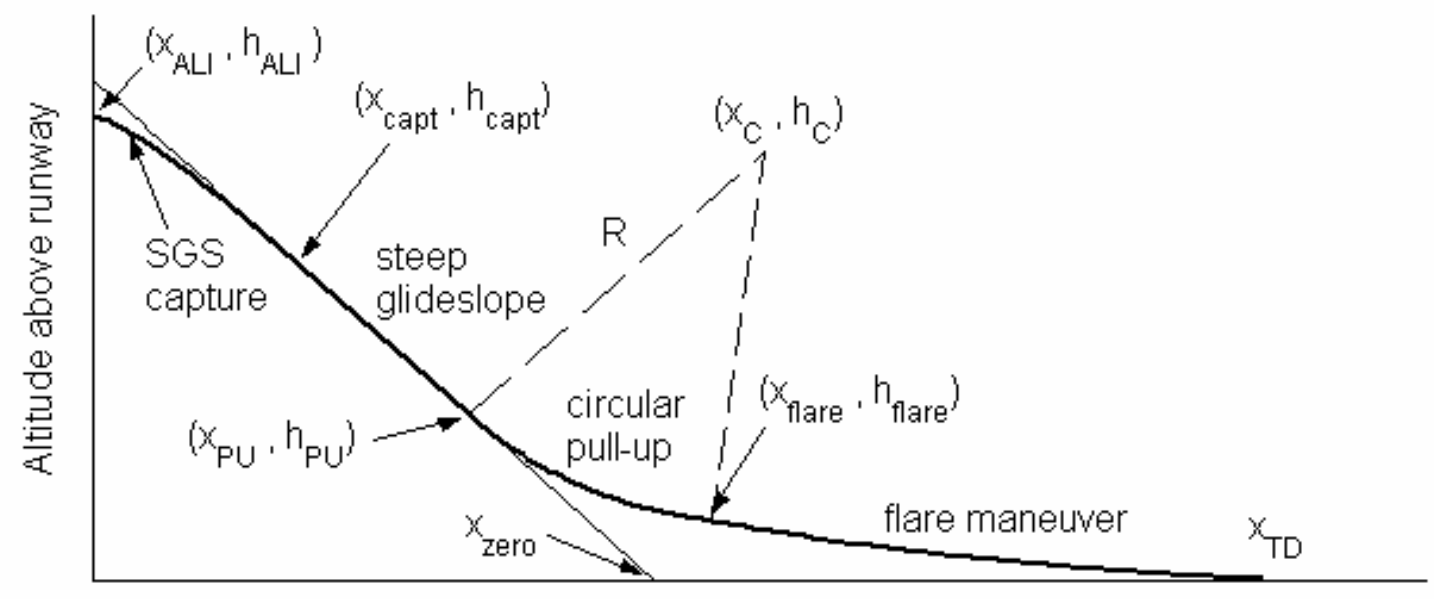

Downrange position

Figure 1-18: Approach and Landing altitude profile with respect to downrange.

\subsubsection{Steep Glideslope Phase}

Following an equilibrium glide slope at maximum L/D generally cannot be maintained without introducing phugoid oscillations because of dynamic pressure changes when a vehicle gets lower in altitude. Therefore, Kluever employs a "quasi-equilibrium glide" (QEG) condition, Equation (1-56) and Equation (1-57), to keep dynamic pressure and glide path angle constant during a steep glideslope phase.

$$
\dot{\gamma}=\frac{L}{m V}-\frac{g}{V} \cos \gamma=0
$$




$$
\begin{gathered}
\dot{\bar{q}}=V \sin \gamma\left[\left(-\beta-\frac{\rho \operatorname{Sref} C_{D}}{m \sin \gamma}\right) \bar{q}-\rho g\right] \\
\text { where } \beta=1 / 30499 f t
\end{gathered}
$$

Where $g$ is gravity magnitude, $m$ is mass, $V$ is relative body velocity magnitude, $L$ is lift force, $\bar{q}$ and $\dot{\bar{q}}$ are the dynamic pressure and a time derivative of dynamic pressure, and $\beta$ is a term proportional to change of density due to change in height.

The altitude profile, $h_{r e f}$, during a steep glideslope phase is linear because a vehicle must maintain a constant steep glideslope angle, $\gamma_{\mathrm{SGS}}$.

$$
h_{r e f}=\tan \gamma_{S G S}\left(x-x_{z e r o}\right)
$$

Where $x$ is the current downrange and $x_{\text {zero }}$ is the downrange location where the steep glideslope intersects the ground, as seen in Figure 1-18. Equation (1-56) is rearranged to solve for the lift coefficient to fly the altitude profile.

$$
C_{L}=\frac{g \cos \gamma_{S G S}}{\bar{q} \operatorname{Sref} / m}
$$

\subsubsection{Circular Pull-Up Phase}

The circular pull up phase smoothes the transition between a steep glideslope angle and a flare angle, $\gamma_{\text {flare }}$, so that transients are eliminated. A vehicle follows a circular pull up altitude profile, described in Equation (1-60) ${ }^{27}$ when downrange, $x$, is less than downrange at the circular pull up downrange, $x_{\mathrm{pu}}$.

$$
h_{\text {ref }}=h_{c}-\sqrt{R^{2}-\left(x-x_{c}\right)}
$$


Where $h_{\mathrm{c}}$ and $x_{\mathrm{c}}$ are altitude and downrange of the origin of the circular pull up, and $R$ is the radius, as depicted in Figure 1-18.

The lift coefficient required to track an altitude profile, $h_{r e f}$, is derived by substituting centripetal acceleration:

$$
V \dot{\gamma}=\frac{V^{2}}{R}
$$

Into the vertical flight path angle dynamic equation, $\dot{\gamma}$ :

$$
\dot{\gamma}=\frac{L}{m V}-\frac{g}{V} \cos \gamma
$$

Solving for $L$, then dividing the quantity by a product of dynamic pressure and reference area ${ }^{27}$ :

$$
C_{L}=\frac{\frac{V^{2}}{R} g \cos \gamma_{r e f}}{\bar{q} \text { Sref } / m}
$$

\subsubsection{Final Flare}

The cubic altitude profile in Equation $(1-64)^{27}$ replaces the standard Space Shuttle flare maneuvers consisting of the exponential decay of the glideslope and a final flare maneuver.

$$
h_{r e f}=a_{0}+a_{1} s+a_{2} s^{2}+a_{3} s^{3}
$$

Ground track distance is denoted by $s$, where $s$ equals 0 when $x=x_{\text {flare }}$. Taking a derivative of Equation (1-64) with respect to ground track gives a tangent of a flight path angle.

$$
\begin{aligned}
\frac{d h_{r e f}}{d s} & =a_{1}+2 a_{2} s+3 a_{3} s^{2} \\
& =\tan \gamma_{r e f}
\end{aligned}
$$


In order to solve for the polynomial coefficients $a_{0}, a_{1}, a_{2}$, and $a_{3}$, the following initial and terminal condition of a final flare phase are used together:

$$
\begin{gathered}
h_{\text {ref }}=h_{\text {flare }} \text { when } s=0 \\
h_{T D}=0 \quad \text { when } s=x_{T D}-x_{\text {flare }}
\end{gathered}
$$

Where subscript, $T D$ represents touchdown conditions, and subscript, flare, represents initial final flare phase conditions. Required lift coefficient to track this altitude profile is represented by Equation $(1-68)^{27}$.

$$
C_{L}=\frac{\cos \gamma_{r e f}}{\bar{q} \operatorname{sref} / m}\left[V^{2}\left(2 a_{2}+6 a_{3} s\right) \cos ^{2} \gamma_{r e f}+g\right]
$$

Equation (1-68) is derived by first taking the time derivative of Equation (1-65), as seen in Equations (1-69) and (1-70).

$$
\begin{aligned}
\frac{d}{d t}\left(\tan \gamma_{r e f}\right) & =\frac{d}{d s}\left(\tan \gamma_{r e f}\right) \frac{d s}{d t} \\
\frac{d}{d t}\left(\tan \gamma_{r e f}\right) & =\left(1+\tan ^{2} \gamma_{r e f}\right) \frac{d \gamma}{d t} \\
& =\left(2 a_{2}+6 a_{3} s\right) \frac{d s}{d t}
\end{aligned}
$$

The dynamic equation for flight path angle, Equation (1-62), is substituted with the left side of Equation (1-69), and $\frac{d s}{d t}$ from the right side of Equation (1-69) is substituted with:

$$
\dot{x}=V \cos \gamma
$$

Then the substituted equation is rearranged and solved for lift to give Equation (1-68). 


\subsubsection{Trajectory Propagation}

A reference trajectory is computed by first solving for a steep glideslope bounded by the QEG conditions from Equations (1-56) and (1-57). Following steps are used to iterate on a steep glideslope angle until the QEG condition is satisfied ${ }^{27}$ :

1. Make an initial guess of a steep glideslope angle, $\gamma_{S G S}$. Select a representative altitude, $h_{r e f}$, to calculate atmospheric density, $\rho_{r e f}$, and speed of sound, $a_{r e f}$, used in all subsequent equations. $h_{r e f}$ is chosen to be $5,000 \mathrm{ft}$, which is half of the altitude at ALI, $h_{\mathrm{ALI}}$

2. Solve for dynamic pressure, Qbar, using the reference density and ALI velocity. Using Equation (1-59) solve for a desired lift coefficient needed to achieve a constant flight path angle, use this lift coefficient in all of the subsequent equations.

$$
Q b a r=\frac{1}{2} \rho_{r e f} v_{A L I}^{2}
$$

3. Resolve for Qbar using the following equation:

$$
Q b a r=\frac{m g \cos \gamma}{C L_{\text {des }} \operatorname{Sref}}
$$

4. Compute the Mach number using the Qbar solved in Step 3 and the density and speed of sound values from the reference altitude.

$$
\begin{aligned}
& V=\sqrt{2 \frac{Q b a r}{\rho_{\text {ref }}}} \\
& \text { Mach }=\frac{V}{a_{\text {ref }}}
\end{aligned}
$$

5. Do a reverse table look up of the static CL tables. Find $\alpha$ that will produce a desired $C L$ from Step 2 with Mach number computed from Step 4 and setting $\beta=0: \alpha=f c n(C L$, Mach, $\beta$ ) 
6. Calculate the corresponding static drag coefficient from the drag tables using $\alpha$ solved in Step 5, Mach solved from Step 4, and setting $\beta=0: C D=f c n(\alpha$, Mach, $\beta)$

7. Use Equation (1-57) to calculate Qdot, $\dot{\bar{q}}$

8. If the absolute value of Qdot is greater than a tolerance, 1e-6, then use a secant line search to adjust $\gamma_{\text {sgs. }}$ Repeat steps 3 through 7 until Qdot converges within the tolerance.

After $\gamma_{\mathrm{SGS}}$ is computed, a trajectory is propagated backward from the start of a final flare phase to the end of a steep glideslope phase. Equations (1-76) through (1-79) are governing equations used to numerical integrate a trajectory backward.

$$
\begin{gathered}
\dot{V}=\frac{-D}{m}-g \sin \gamma \\
\dot{\gamma}=\frac{L}{m V}-\frac{g}{V} \cos \gamma \\
\dot{h}=V \sin \gamma \\
\dot{x}=V \cos \gamma
\end{gathered}
$$

The backward integration is constrained by boundary conditions specified by a desired touchdown condition, ALI conditions, and $\gamma_{\mathrm{SGS}}$ previously solved. Touchdown altitude, velocity, and sink rate is a fixed value. $\gamma_{\mathrm{TD}}$ can be computed by using Equation (1-78) so that touchdown conditions are fully defined.

Altitude and vertical flight path angle at an initial point of a final flare maneuver, $\gamma_{\text {flare }}$, and total ground track of a flare phase, $s_{f f a r e}$, can be used as tuning parameters to shape an altitude profile. However, initial flare altitude, $h_{\text {flare }}$, is fixed to $150 \mathrm{ft}$ to simplify the algorithm so that only $\gamma_{\text {flare }}$ is used as a free tuning parameter to iterate upon. Total ground track for a final flare 
phase is calculated by using Equation $(1-80)^{27}$, which is derived from Equation (1-64) and Equation (1-65) after solving for maximum range.

$$
s_{\text {flare }}=\frac{3\left(h_{T D}-h_{\text {flare }}\right)}{2 \tan \gamma_{\text {flare }}+\tan \gamma_{\text {flare }}}
$$

Velocity is controlled implicitly through a selection of $\gamma_{\text {flare, }}$, which also determines an initial slope of a cubic altitude profile and $s_{f l a r e}$ used in a backward integration for a flare phase. The following steps ${ }^{27}$ below are used to iterate on $\gamma_{\text {flare }}$ until a backward propagation of a trajectory is continuous in altitude, $\gamma$, and Qbar.

1. Backward propagation from touchdown to start of final flare: Choose a $\gamma_{\text {flare }}$ value and solve for Equation (1-80). Divide Equations (1-76) through (1-78) by Equation (1-79); and take an inverse of Equation (1-79), so now the differential equations are $d V / d x, d \gamma / d x, d h / d x, d t / d x$. The independent state used for backward integration will be downrange, $x$. In order to solve for $d \gamma / d x$ the lift has to be solved using Equation (1-68).The drag from $d V / d x$ could be solved from the aerodynamic tables.

2. Backward integration from circular pull up to steep glideslope: Use state values obtained from Step 1 to initialize backward propagation from the end of the pull up to ensure continuity. The end of a pull up phase has the same condition as the start of a final flare phase to make a trajectory continuous. Solve for a pull up radius using Equation (1-63) with states from the start of the final flare. Divide Equations (1-76), (1-78), and (1-79), by Equation (1-77) and invert Equation (1-77) to get: $d V / d \gamma, d x / d \gamma, d t / d \gamma$. Take a derivative of Equation (1-60) with respect to $\gamma$ in order to get $d h / d \gamma$. Use $\gamma$ as an independent variable to integrate differential equations. Use Equation (1-63) to solve for lift, and use aerodynamic tables to solve for drag for the same flight condition. 
3. Qbar Check: Check to see whether Qbar from the initial pull up equals the quasiequilibrium Qbar solved from the steep glideslope phase. If not, repeat Steps 1 and 2. Once Qbar matches proceed to Step 4.

4. Forward integration of steep glideslope phase: To complete a trajectory, compute a forward integration from ALI to the steep glideslope phase. Divide Equation (1-76), (1-77), and (1-79) by Equation (1-78), and invert Equation (1-78) to get: $d V / d h, d \gamma / d h$, $d x / d h, d t / d h$. Altitude becomes the independent variable for integration.

5. Piece all parts of a trajectory from ALI to touchdown.

Steps to compute a continuous landing trajectory is summarized in Figure 1-19.

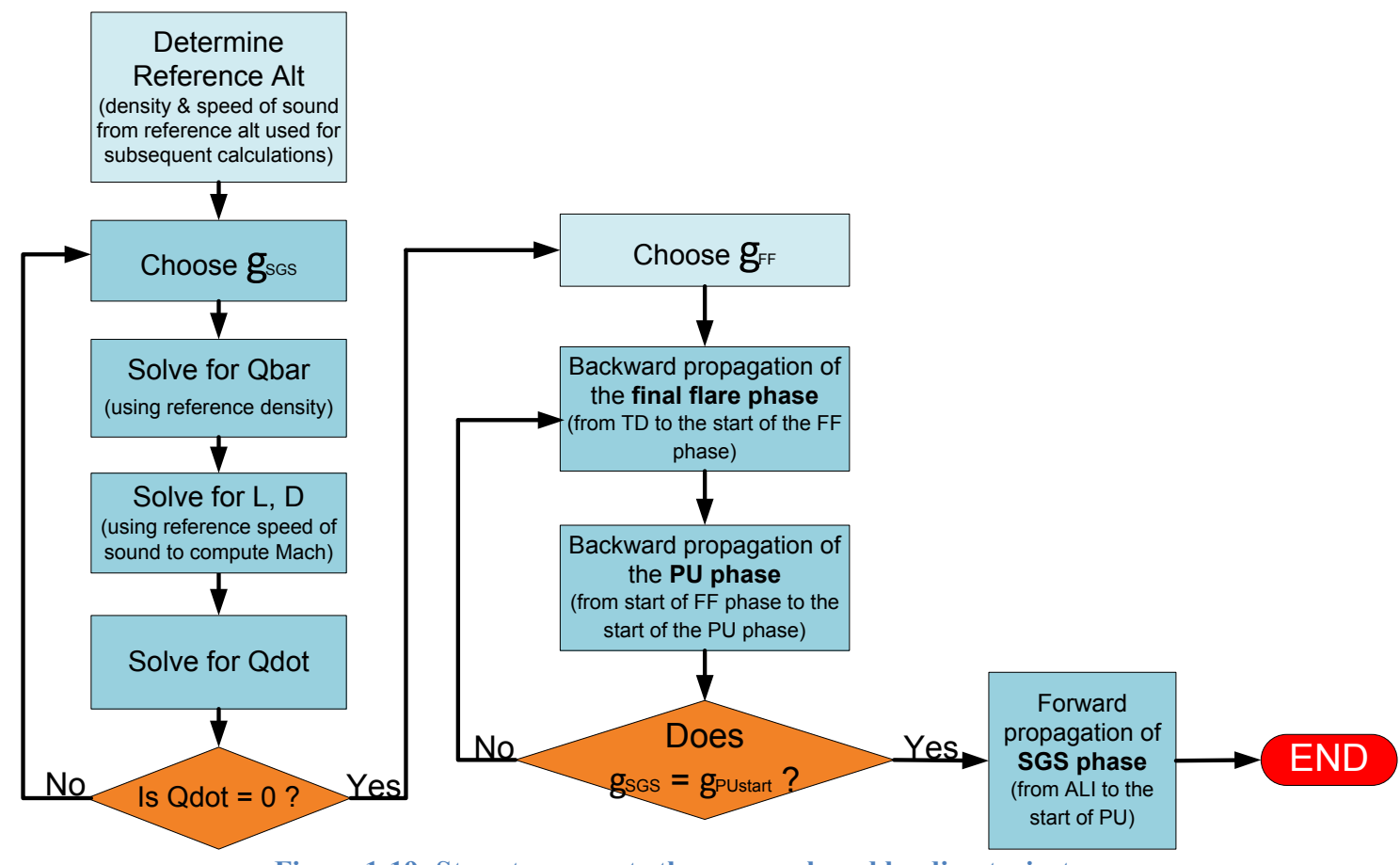

Figure 1-19: Steps to compute the approach and landing trajectory. 


\subsubsection{Computed Reference Trajectory}

Table 1-6 summarizes ALI conditions, TD conditions, and constant parameters used to propagate an approach and landing trajectory. These approach and landing reference velocities were taken from space shuttle conditions.

Table 1-6: Approach and Landing Interface and Touchdown Conditions.

\begin{tabular}{|c|c|c|}
\hline Condition & Value & Units \\
\hline $\mathbf{h}_{\mathrm{ALI}}$ & 10000 & $\mathrm{ft}$ \\
\hline $\mathbf{V}_{\mathrm{ALI}}$ & 621.867 & $\mathrm{ft} / \mathrm{sec}$ \\
\hline $\mathbf{h}_{\text {ref }}$ (for SGS phase) & 5000 & $\mathrm{ft}$ \\
\hline $\mathbf{h}_{\text {TD }}$ & 0 & $\mathrm{ft}$ \\
\hline $\mathbf{V}_{\mathrm{TD}}$ & 314 & $\mathrm{ft} / \mathrm{sec}$ \\
\hline Sink Rate $_{\mathrm{TD}}$ & -2 & $\mathrm{ft} / \mathrm{sec}$ \\
\hline
\end{tabular}

The RLV should follow the trajectory closely since the vehicle's static aerodynamics are taken into consideration as long as $\beta$ and $\mu$ is kept constant at zero degrees. Appendix A contains scripts and functions developed from formulations in Section 1.7.2. Figure 1-20 through Figure 1-24 show a reference trajectory computed from the algorithm that will be used in the approach and landing simulation. 


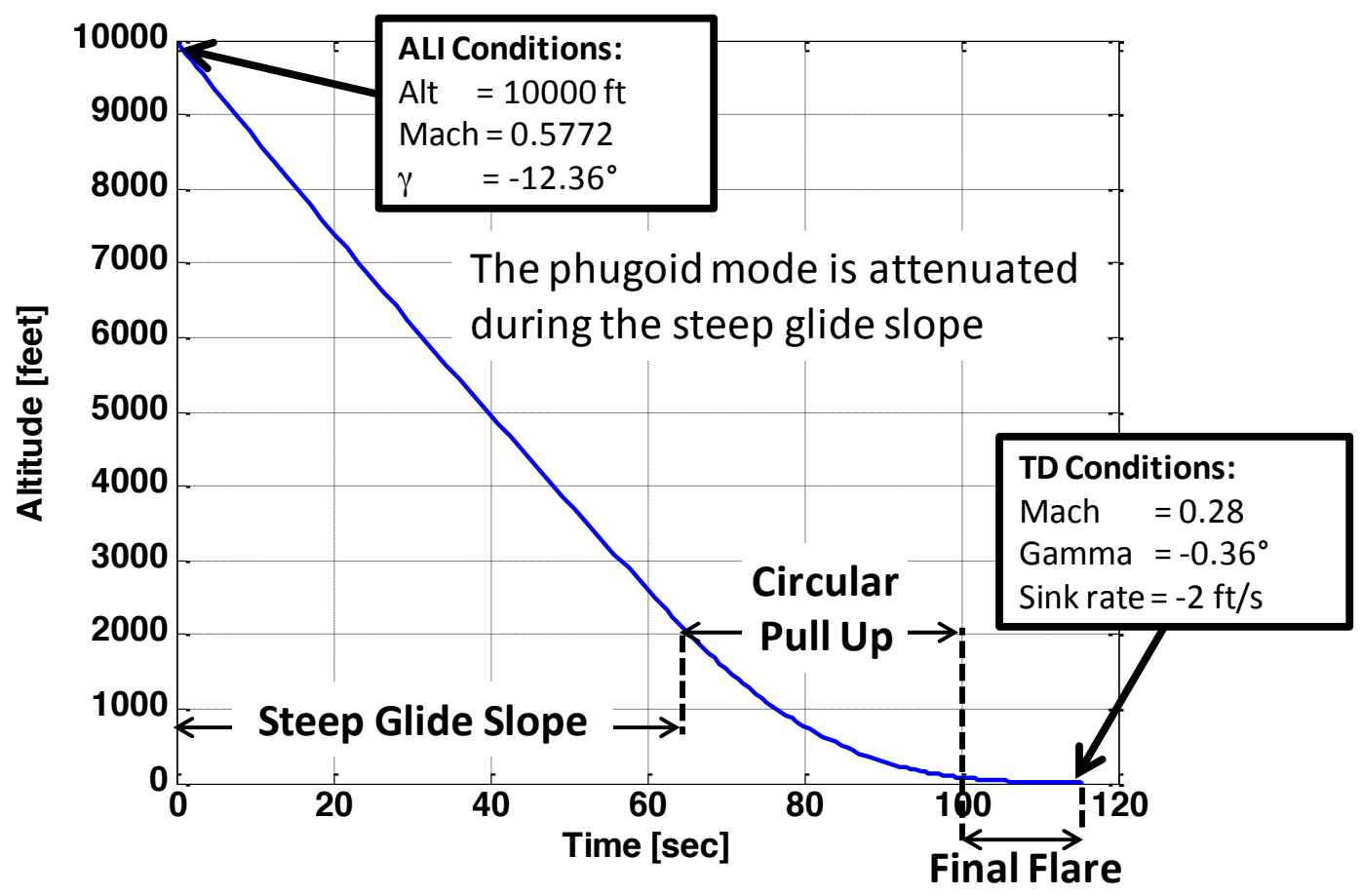

Figure 1-20: Altitude profile.

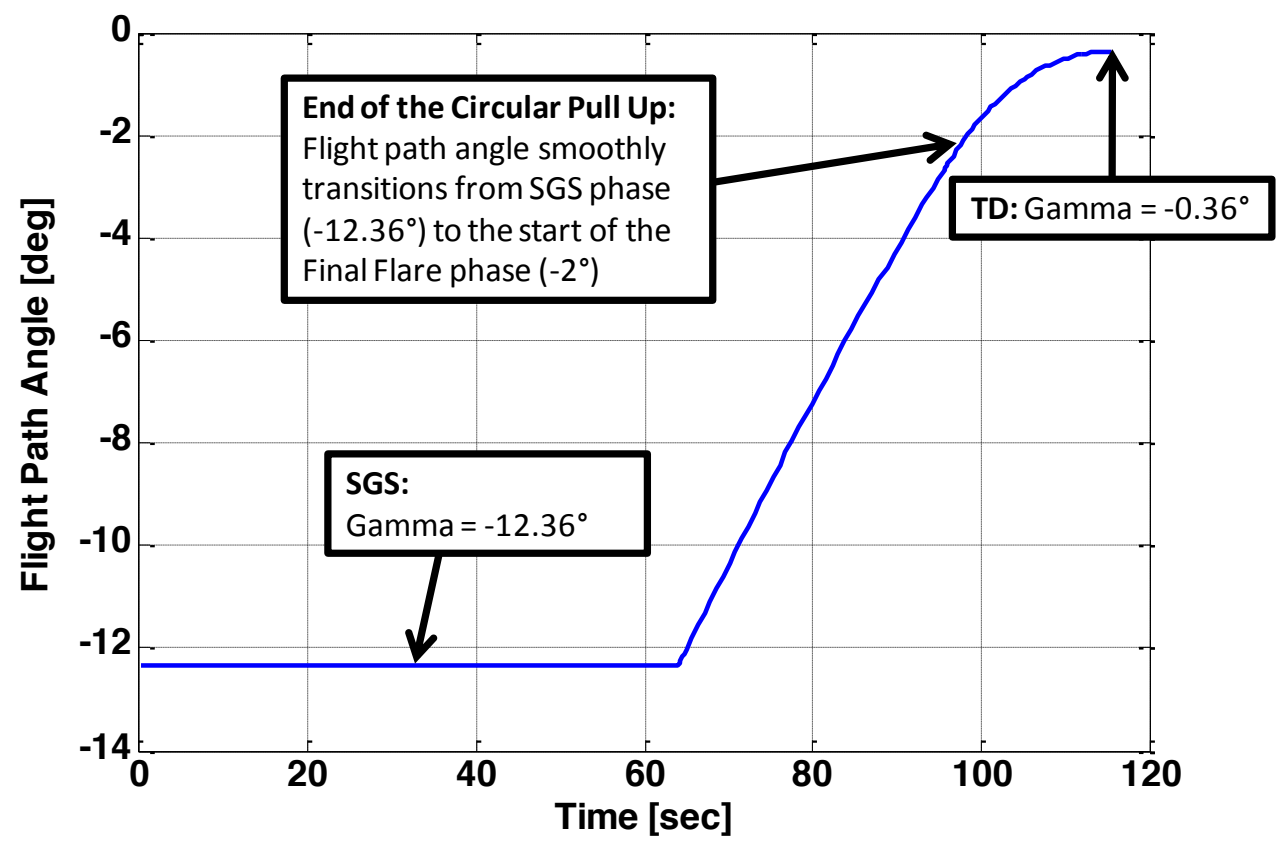

Figure 1-21: Vertical flight path angle profile. 


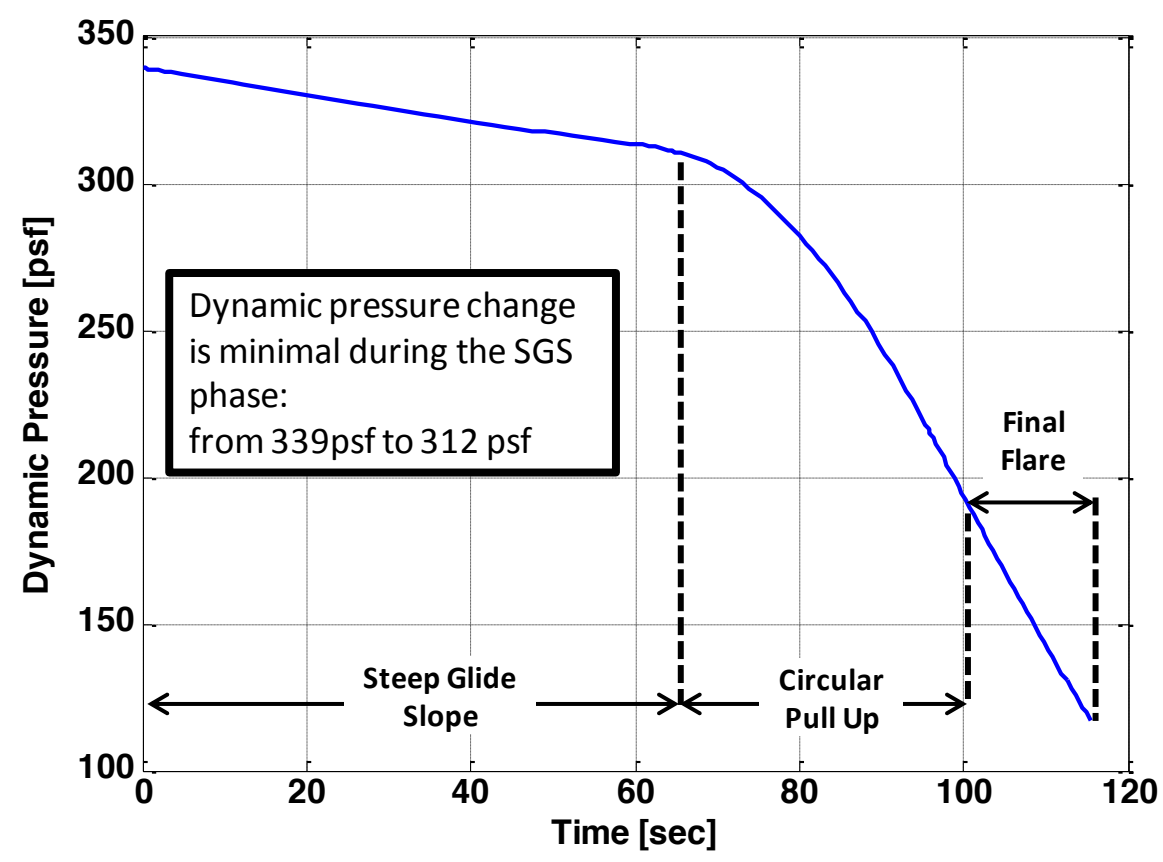

Figure 1-22: Dynamic pressure profile.

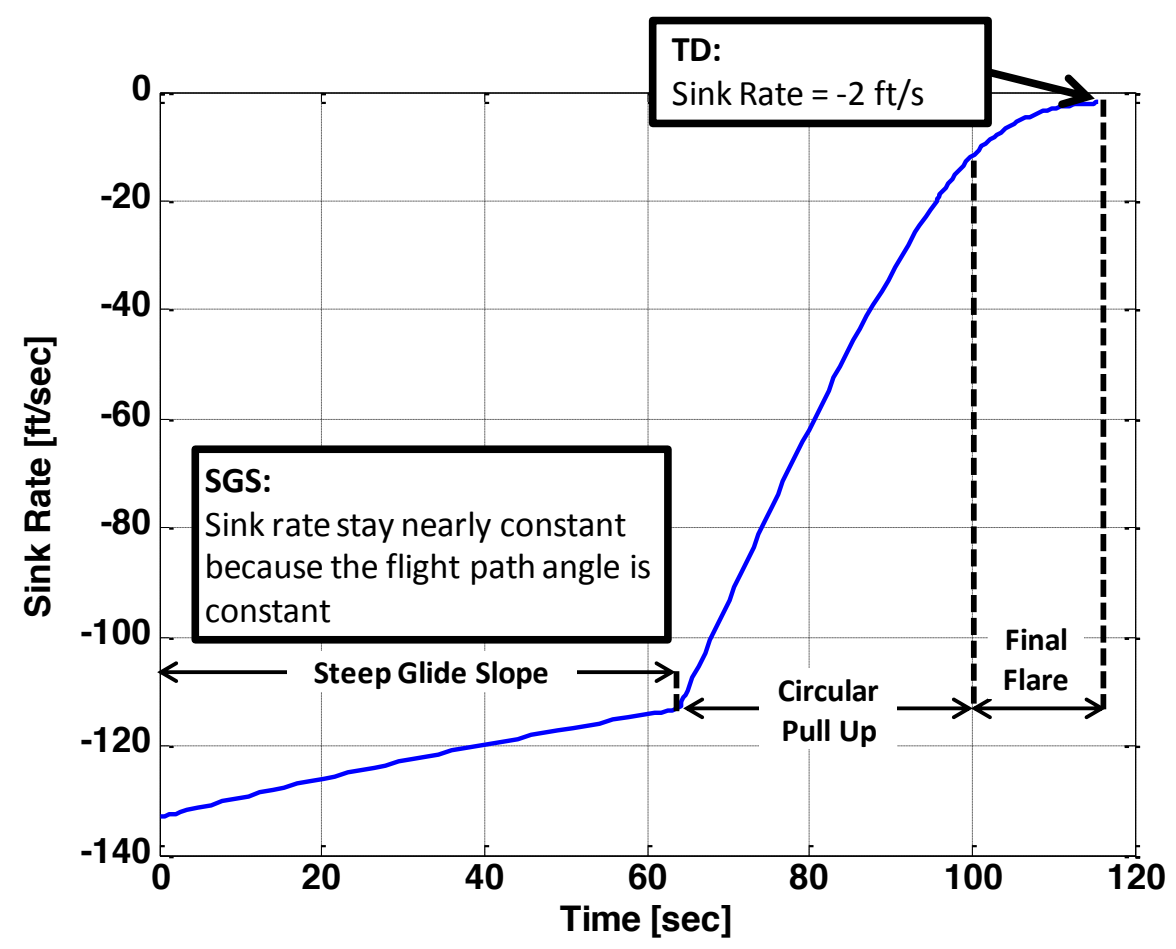

Figure 1-23: Sink rate achieved for the landing profile. 


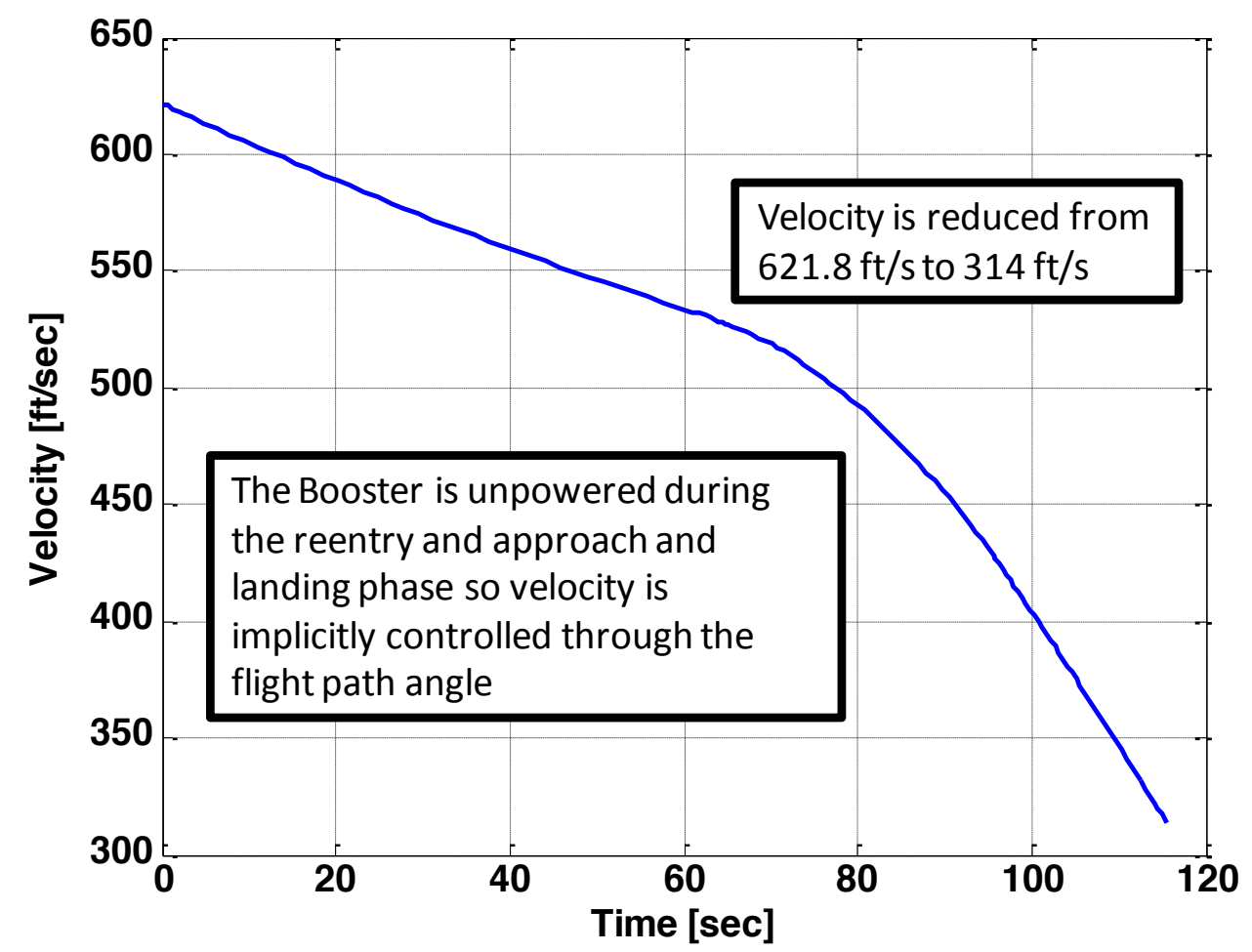

Figure 1-24: Velocity profile during approach and landing.

\subsubsection{Implementation of the Reference Trajectory into Simulink}

Altitude, $\gamma, \dot{\gamma}$ profile are needed as input commands into a backstepping algorithm which will be described in Section 2.2.3.1. The trajectory will be interpolated based on time as an independent variable to compute a point trajectory command for a particular time in the simulation. Pre-look up and look up tables, shown in Figure 1-25, were used to interpolate trajectory commands based on simulation time. 


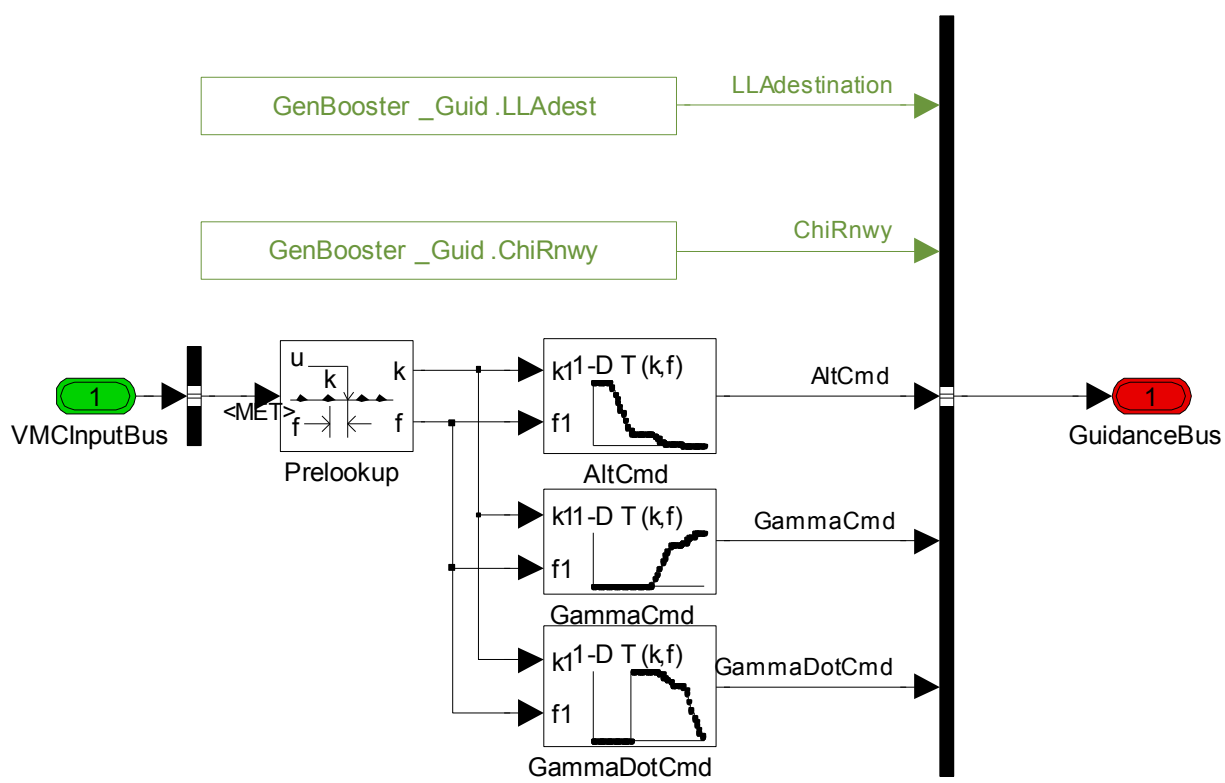

Figure 1-25: Guidance trajectory implementation into Simulink.

The Kennedy Space Center (KSC) was chosen as a desired landing location because it is also the Space Shuttle's desired touchdown location. Table 1-7 shows the KSC position and runway heading used for simulation.

Table 1-7: KSC Landing Parameters Used as the Landing Site for the Trajectory.

\begin{tabular}{|l|c|c|}
\hline \multicolumn{1}{|c|}{$\begin{array}{c}\text { KSC Runway } \\
\text { Parameter }\end{array}$} & Values & Units \\
\hline Latitude & 28.4668 & $\mathrm{deg}$ \\
Longitude & 279.4415 & $\mathrm{deg}$ \\
Altitude & 0 & $\mathrm{ft}$ \\
Runway Heading & 150 & $\mathrm{deg}$ \\
\hline
\end{tabular}

\subsection{Lyapunov Stability}

Robustness measures the stability of a system in the presence of modeling errors. Lyapunov theorems help prove robustness of a dynamic system by quantifying system stability with respect to inputs. Stability describes how states of a system remain bounded for bounded control inputs. Control Lyapunov functions (CLF) are a way to analyze whether a system can be 
stabilized with feedback by applying a control function. CLF are used to prove stability for parts of a system with the dynamic inversion and backstepping control law; so the basics of Lyapunov stability are explained in this section to help understand Lyapunov stability derivations for control algorithms ${ }^{19}$ in later sections.

A system is considered controlled and stable when states are driven toward equilibrium. Lyapunov theory can be used to prove stability for a time-invariant system described as:

$$
\dot{x}=f(x)
$$

Where $x_{e}$ is assumed to be the equilibrium point where $f\left(x_{e}\right)=0$, and $x(0)$ is the initial state. The equilibrium point is:

1. Stable: if for each $\varepsilon>0$ there exists $\delta(\varepsilon)>0$ such that

$$
\left\|x(0)-x_{e}\right\|<\delta \Rightarrow\left\|x(t)-x_{e}\right\|<\varepsilon \text { for all } t \geq 0
$$

2. Asymptotically Stable: if it is stable and in addition there exists $r>0$ such that

$$
\left\|x(0)-x_{e}\right\|<r \Rightarrow x(t) \rightarrow x_{e} \text { as } t \rightarrow \infty
$$

3. Globally Asymptotically Stable (GAS): if it is asymptotically stable for all initial states

A Lyapunov function, $V(x)$, characterizes energy contained in a system by condensing all states into a scalar function. A system is moving toward equilibrium if $V(x)$ can be proven to continuously decrease $^{20}$. A Lyapunov function is used in the LaSalle-Yoshizawa theorem ${ }^{19}$ to prove stability of a system. 


\subsubsection{LaSalle Yoshizawa Theorem}

For a system described in Equation (1-81), let $x=0$ be an equilibrium point and let a Lyapunov function, $V(x)$ be:

1. Scalar

2. Continuously differentiable in state, $x$

3. Positive definite: $V(0)=0$ and $V(x)>0, x \neq 0$

4. Radially unbounded: $V(x) \rightarrow \infty$ as $\|x\| \rightarrow \infty$

5. $\dot{V}(x)=\frac{\partial V}{\partial x} f(x) \leq-W(x)$; where $\mathrm{W}(\mathrm{x})$ is positive semidefinite

If a Lyapunov function satisfies all of the requirements above, then all solutions from a system from Equation (1-81) will satisfy ${ }^{20}$ :

$$
\lim _{t \rightarrow \infty} W(x(t))=0
$$

Moreover, if $W(x)$ is positive definite, then $x=0$ is GAS.

\subsubsection{Lyapunov Control Function}

The La-Salle Yoshizawa theorem will be used to prove stability for a control Lyapunov function. A system with a control input is defined as Equation (1-85) with a control law described in Equation (1-86).

$$
\begin{gathered}
\dot{x}=f(x, u) \\
u=k(x)
\end{gathered}
$$


Then a closed loop system will be defined as Equation (1-87).

$$
\dot{x}=f(x, k(x))
$$

Controller, $k(x)$, has to be chosen so a system is GAS, satisfying Equation (1-88), assuming the origin to be an equilibrium state.

$$
\begin{aligned}
\dot{V}(x) & =\frac{\partial V}{\partial x} f(x, u) \\
& =-W(x)<0 \text { for some } u
\end{aligned}
$$

Where $V(x)$ is a CLF and $W(x)$ is positive definite as defined in the previous section, 1.8.1. $V(x)$ is positive definite and radially unbounded in order to be classified as a CLF ${ }^{20}$. Artstein's theorem states that an existence of a CLF is equivalent to an existence of a globally stabilizing control law. Each controller formulation will prove GAS by creating a CLF for parts of the system.

\subsection{Robustness Test Matrix Description}

The scope of this thesis is to test robustness of a backstepping and dynamic inversion controller to unmodeled plant uncertainties, which is encountered through an RLV's wide flight envelope. Following sections will describe different types of aerodynamic uncertainties that are inserted for various test cases. Analysis for these test cases will determine which type of uncertainties affect robustness of the controller and how much uncertainty can be added without degrading controller performance to a point of damaging a vehicle or losing control.

\subsubsection{Aerodynamic Uncertainties}

Aerodynamic uncertainties are modeled for static aerodynamics and control surface aerodynamics. The static aerodynamics are provided in a three-dimensional table as a function of 
$\alpha$, Mach, and $\beta$, as described in Equation (1-44). Control surface aerodynamics are provided for all seven control surfaces as four-dimensional tables as functions of $\alpha$, Mach, $\beta$, and $\delta$, seen in Equation (1-50). The original NGC provided aerodynamic tables will be considered the nominal aerodynamic data. Uncertainties are represented as a constant percentage of the nominal aerodynamic data added or subtracted, depending on the sign of the percentage, to each element in the nominal tables. Therefore, the perturbation about the nominal aerodynamic data remains constant throughout the vehicle's flight envelope. A randomly generated constant corresponding to a fraction equivalent to an uncertainty percentage range of interest is created. This random constant is multiplied with all elements within the nominal aerodynamic tables to attain a table of parameters representing a percentage of the nominal aerodynamic data. The table of parameters are added to the nominal aerodynamic table to represent aerodynamic uncertainties within a certain percentage range of a plant's aerodynamics.

For example, a $+/-30 \%$ static aerodynamic uncertainty for a roll coefficient, $\mathrm{Cl}$, uncertainty is modeled by using the "rand" function in Matlab. The "rand" function returns a uniformly distributed random fraction varying from -0.30 to +0.30 . This random fraction is multiplied to the nominal aerodynamic table to a generate a matrix containing values between $-30 \%$ and $+30 \%$ of the actual $\mathrm{Cl}$ across the flight envelope. This matrix product is added to the nominal table to generate an aerodynamic table representing $+/-30 \%$ plant uncertainty, as depicted in Figure 1-26. A similar process is implemented for each three-dimensional static aerodynamic table and each four-dimensional control surface aerodynamic table. Since fractions for each table are randomly generated between an interval, the perturbation about the nominal $\mathrm{Cl}$ may be different from the perturbation about the nominal $\mathrm{Cm}$ for a particular simulation run for a $+/-30 \%$ static aerodynamic uncertainty case. 


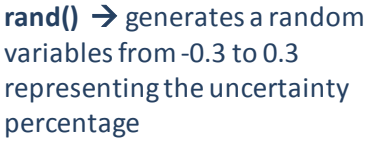

0.21
Uncertainty value of the aerodynamic term $\rightarrow$ Do an element wise multiplication with the original aerodynamic table
Aerodynamic table with uncertainty $\rightarrow$ Add the uncertainty aerodynamic value to the original data

\section{Figure 1-26: Process to insert uncertainty into an aerodynamic table.}

Uncertainties of $+/-10 \%,+/-20 \%,+/-30 \%,+/-40 \%,+/-50 \%,+/-60 \%$ are added to each aerodynamic coefficient table for static aerodynamics and control surface aerodynamics for each control surface. These tables are saved as mat-files that are loaded into table look-ups used in the Plant Aerodynamics block to compute the RLV's aerodynamic coefficients in the plant model, represented in Figure 1-14. Nominal aerodynamic data is retained for aerodynamic models in the controller. Uncertainty is simulated when aerodynamic forces and moments between the plant model and the controller differed.

\subsubsection{Test Cases}

A test matrix, displayed in Table 1-8 is created to analyze robustness characteristics of a dynamic inversion controller alone and a dynamic inversion controller together with a backstepping controller for varying aerodynamic uncertainties. The test matrix consists of four subsets of test objectives that are performed for each aerodynamic uncertainty type implemented in the Plant. These test cases include performing body rate (PQR) doublets and an approach and landing trajectory tracking. Each test case inserts aerodynamic uncertainties one type at a time: first, static aerodynamic uncertainties only, then, control surface aerodynamic uncertainties only, and finally, both aerodynamic uncertainties together. This helps pin point which type of aerodynamic uncertainties affect robustness of a dynamic inversion controller with and without a backstepping controller, and whether having uncertainties in both aerodynamic terms will compound effects on robustness. For body rate doublet cases aerodynamic uncertainties of 
$+/-30 \%,+/-40 \%,+/-50 \%$, and $+/-60 \%$ were tested. Originally, the approach and landing trajectory tracking was tested with $+/-30 \%$ aerodynamic uncertainties. However, most simulation run resulted in the RLV losing control. Therefore, trajectory tracking is tested with $10 \%$ aerodynamic uncertainties first, then increased to $20 \%$ to quantify controller robustness.

Table 1-8: Test Matrix used for the robustness analysis.

\begin{tabular}{|c|c|c|c|c|}
\hline Uncertainty Types & $\begin{array}{c}\text { Flight } \\
\text { Condition } \\
\end{array}$ & Test Case & $\begin{array}{c}\text { Simulation } \\
\text { Runs } \\
\end{array}$ & Remarks \\
\hline \multirow{3}{*}{$\begin{array}{l}30 \% \text { Static Uncertainty ONLY, } \\
30 \% \text { Control Uncertainty ONLY, } \\
30 \% \text { Static \& Control Uncertainty }\end{array}$} & A & P Doublet & 300 & $\begin{array}{l}\text { Test Dynamic inversion } \\
\text { robustness in roll axis }\end{array}$ \\
\hline & A & Q Doublet & 300 & $\begin{array}{l}\text { Test Dynamic inversion } \\
\text { robustness in pitch axis }\end{array}$ \\
\hline & A & R Doublet & 300 & $\begin{array}{l}\text { Test Dynamic inversion } \\
\text { robustness in yaw axis }\end{array}$ \\
\hline $\begin{array}{l}10 \% \text { Static ONLY, } 10 \% \text { Control } \\
\text { ONLY, } 10 \% \text { Static \& Control, } \\
20 \% \text { Static ONLY, } 20 \% \text { Control } \\
\text { ONLY, } 20 \% \text { Static \& Control }\end{array}$ & B & $\begin{array}{l}\text { Approach and } \\
\text { Landing } \\
\text { Trajectory }\end{array}$ & 300 & $\begin{array}{l}\text { Test dynamic inversion } \\
\text { and backstepping } \\
\text { controller robustness }\end{array}$ \\
\hline \multirow{3}{*}{$\begin{array}{l}40 \% \text { Static Uncertainty ONLY, } \\
40 \% \text { Control Uncertainty ONLY, } \\
40 \% \text { Static \& Control Uncertainty }\end{array}$} & A & P Doublet & 350 & $\begin{array}{l}\text { Test Dynamic inversion } \\
\text { robustness in roll axis }\end{array}$ \\
\hline & A & Q Doublet & 350 & $\begin{array}{l}\text { Test Dynamic inversion } \\
\text { robustness in pitch axis }\end{array}$ \\
\hline & A & R Doublet & 350 & $\begin{array}{l}\text { Test Dynamic inversion } \\
\text { robustness in yaw axis }\end{array}$ \\
\hline \multirow{3}{*}{$\begin{array}{l}50 \% \text { Static Uncertainty ONLY, } \\
50 \% \text { Control Uncertainty ONLY, } \\
50 \% \text { Static \& Control Uncertainty }\end{array}$} & A & P Doublet & 550 & $\begin{array}{c}\text { Test Dynamic inversion } \\
\text { robustness in roll axis }\end{array}$ \\
\hline & A & Q Doublet & 550 & $\begin{array}{l}\text { Test Dynamic inversion } \\
\text { robustness in pitch axis }\end{array}$ \\
\hline & A & R Doublet & 550 & $\begin{array}{l}\text { Test Dynamic inversion } \\
\text { robustness in yaw axis }\end{array}$ \\
\hline \multirow{3}{*}{$\begin{array}{l}60 \% \text { Static Uncertainty ONLY, } \\
60 \% \text { Control Uncertainty ONLY, } \\
60 \% \text { Static \& Control Uncertainty }\end{array}$} & A & P Doublet & 650 & $\begin{array}{l}\text { Test Dynamic inversion } \\
\text { robustness in roll axis }\end{array}$ \\
\hline & A & Q Doublet & 650 & $\begin{array}{l}\text { Test Dynamic inversion } \\
\text { robustness in pitch axis }\end{array}$ \\
\hline & A & R Doublet & 650 & $\begin{array}{l}\text { Test Dynamic inversion } \\
\text { robustness in yaw axis }\end{array}$ \\
\hline
\end{tabular}

Multiple simulation runs are conducted for each test case, depending on the magnitude of an uncertainty percentage, in order to characterize controller robustness. Number of simulation runs are determined using a probability theory called the Law of Large Numbers. The probability 
theorem states that an average of independent outcomes of a random variable that have the same probability distribution will be close to the expected value. If all outcomes are equally likely, then the expected value will equal the mean. Law of Large Numbers ensures stable long-term results for random events if the number of simulation runs for a test case is sufficiently large. For example, the "rand" function will produce any uncertainty fraction between -0.3 and +0.3 for a simulation run for any $+/-30 \%$ uncertainty case. The more simulation runs conducted for the same test case, the more likely the sample average of uncertainties for all simulation runs will equal to zero, the mean of -0.3 and +0.3 . Figure 1-27 illustrates the convergence of a sample mean for the $+/-30 \%$ uncertainty case for a static aerodynamic term, $\mathrm{Cl}$. Law of Large Numbers is a way to determine the likely performance of the controller based on the outcomes of random sampling between the uncertainty percentage range.

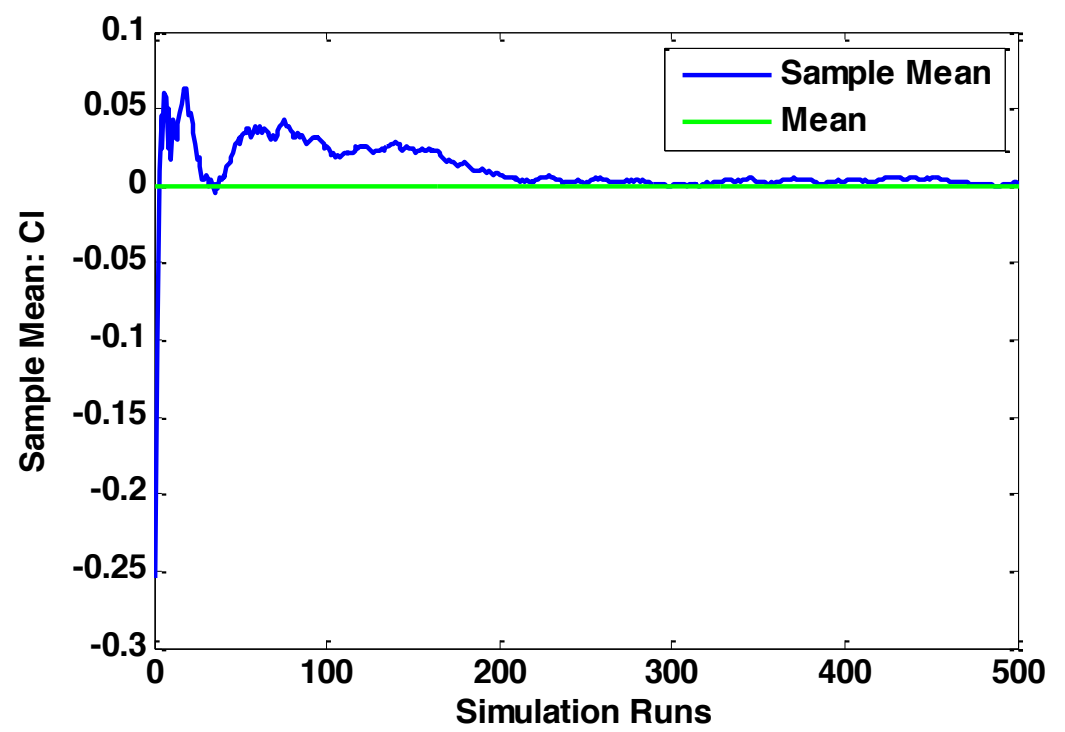

Figure 1-27: Sample mean convergence to zero for the $+/-30 \%$ Uncertainty Case.

The number of simulation runs is determined to be 300 for any $+/-10 \%,+/-20 \%,+/-30 \%$ uncertainty case, assuming that other static aerodynamic coefficients and control surface aerodynamic coefficients contain the same range of uncertainty and random numbers generated 
from the same uniform distribution. The resulting number of simulation runs for a $+/-40 \%$, $+/-50 \%$, and $+/-60 \%$ uncertainty case is 350 runs, 550 runs, and 650 runs, respectively, when the Law of Large Numbers is applied. The simulation runs were increased until the sample average reached between -0.05 and +0.05 and the standard deviation between each coefficient stayed within 0.02 of each other.

\subsubsection{Body Rate (PQR) Doublets}

The purpose of performing PQR doublets is to quantify robustness of a dynamic inversion controller only. A doublet is conducted for one axis at a time, while commanding zero deg/sec for other axes. Figure 1-28 depicts a doublet performed for each axis. Positive and negative maneuvers are tested because control surface aerodynamics are not linear so a positive and negative command can affect the controller performance differently. The yaw axis doublet is much lower in magnitude than for the roll and pitch axis because a yawing maneuver induces a $\beta$ which affects the RLV's directional stability. Too much $\beta$ will cause the vehicle to become too unstable to control without saturating control surfaces.

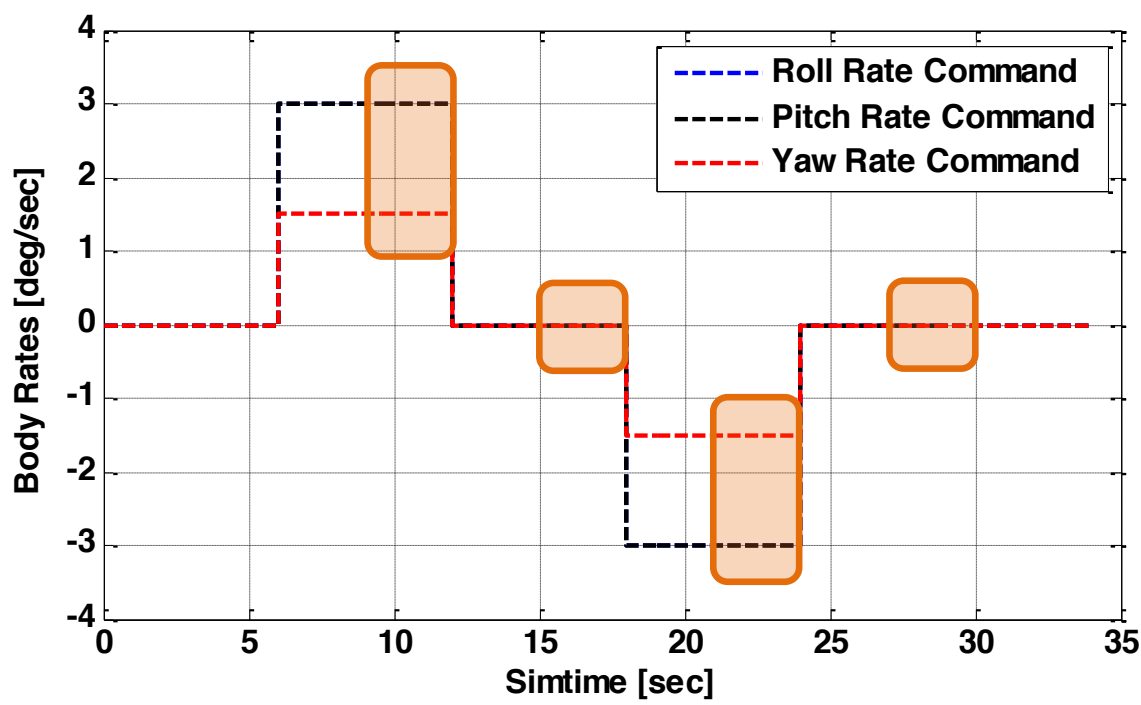

Figure 1-28: Body rate doublet commands fed into the dynamic inversion controller. 
Steady state errors used to quantify controller performance are taken at points circled in the figure above. Steady state error is taken after a couple seconds after a step command is initiated to allow the controller to have time to achieve the command. The steady state error is defined as the error between the commanded value and the standard deviation of the controller performance for the total number of simulation runs conducted. A standard deviation contains about $68 \%$ of states within the mean, so vehicle states are more likely to fall within this range given the amount of random plant uncertainty.

Flight condition is frozen for these test cases in order to take out transient effects caused by the vehicle's motion changing dynamic pressure and Mach number. Moreover, the RLV is unpowered so the only way to retain energy during the doublet maneuver is to freeze a flight condition artificially in the simulation. Flight condition is frozen by zeroing out the acceleration vector feeding into an acceleration integrator, and zeroing out the velocity going into a velocity integrator from Figure 1-17 in the translation equation of motion model. Freezing a flight condition will prevent the vehicle from moving in three-dimensional space, but allow it to rotate about that flight condition point, which means $\mathrm{P}, \mathrm{Q}, \mathrm{R}, \alpha, \beta$, and $\mu$ could change, but the latitude, longitude, altitude, velocity, and flight path angles will stay constant. Table 1-9 describes a flight condition chosen to freeze the simulation to conduct PQR doublets. This flight condition is the same as the initial point in the approach and landing trajectory. 
Table 1-9: Flight Condition used to perform PQR doublet.

\begin{tabular}{|l|c|c|}
\hline Flight Condition & Value & Units \\
\hline Latitude & 28.6088 & $\mathrm{deg}$ \\
Longitude & -80.6515 & $\mathrm{deg}$ \\
Altitude & 10000 & $\mathrm{ft}$ \\
Mach & 0.5772 & $\mathrm{ND}$ \\
Angle of Attack & 3.6 & $\mathrm{deg}$ \\
Bank Angle & 0 & $\mathrm{deg}$ \\
Heading & 150 & $\mathrm{deg}$ \\
Flight Path Angle & -12 & $\mathrm{deg}$ \\
Dynamic Pressure & 339.4 & $\mathrm{psf}$ \\
\hline
\end{tabular}

\subsubsection{Approach and Landing Trajectory}

Trajectory tracking will test robustness of the whole system: the backstepping and dynamic inversion controller with RLV dynamics. A trajectory solved in Section 1.7.3 will be used. Inserting static aerodynamic and control surface aerodynamic uncertainties separately allows us to see which uncertainty type will affect robustness of flight parameters and states for a given trajectory. Robustness of the controller will be analyzed by incrementally increasing the aerodynamic uncertainties for both static and control surface aerodynamics until the controller performance degrades and the vehicle performance is deemed unacceptable.

\subsection{Performance Metrics}

Performance metrics or figures of merits were developed for each test case to quantify the controller performance. Table 1-10 summarizes these figures of merits. Green represents good tracking, yellow represents acceptable tracking, and red represents unacceptable tracking. 
Table 1-10: Figures of merit for the controller tracking performance.

\begin{tabular}{|l|c|c|c|}
\hline \multicolumn{1}{|c|}{ Test Type } & \multicolumn{1}{|c|}{ Figures of Merits } \\
\hline \hline $\begin{array}{l}\text { PQR doublet: } \\
\text { Max absolute steady state } \\
\text { error }(\varepsilon)\end{array}$ & $\begin{array}{c}\varepsilon<=5 \% \\
(\varepsilon<=0.15 \mathrm{deg} / \mathrm{sec})\end{array}$ & $\begin{array}{c}5 \%<\varepsilon<=10 \% \\
(0.15 \mathrm{deg} / \mathrm{sec}<\varepsilon<0.3 \mathrm{deg} / \mathrm{sec})\end{array}$ & $\begin{array}{c}\varepsilon>10 \% \\
(\varepsilon>0.3 \mathrm{deg} / \mathrm{sec})\end{array}$ \\
\hline $\begin{array}{l}\text { Crosstrack: } \\
\text { max absolute error } \\
\text { (Trajectory tracking) }\end{array}$ & max error $<=5 \mathrm{ft}$ & $5 \mathrm{ft}<\max$ error $<=10 \mathrm{ft}$ & $\max$ error $>10 \mathrm{ft}$ \\
\hline $\begin{array}{l}\text { Heading: } \\
\text { max absolute error } \\
\text { (deviation from Runway } \\
\text { Heading of } 150 \text { deg) }\end{array}$ & $<3 \mathrm{deg}$ & $3 \mathrm{deg}<\max$ error $<=5 \mathrm{deg}$ & $<5 \mathrm{deg}$ \\
\hline $\begin{array}{l}\text { Sink rate: at touchdown } \\
\text { (Trajectory tracking) }\end{array}$ & $<-5 \mathrm{ft} / \mathrm{sec}$ & $-5 \mathrm{ft} / \mathrm{sec}<\operatorname{sink}$ rate $<=-10 \mathrm{ft} / \mathrm{sec}$ & $>-10 \mathrm{ft} / \mathrm{sec}$ \\
\hline
\end{tabular}

Parameters involving error calculations refer to the error between the standard deviation and the command. The altitude error is not included in the table since the figures of merit for altitude tracking is dependent on velocity. For faster velocities, any small errors in flight path angle may result in a large altitude error, but for smaller velocities the altitude error will be smaller for the same flight path angle error. Therefore, altitude error performance metrics are proportional to the vehicle velocity as seen in Figure 1-29.

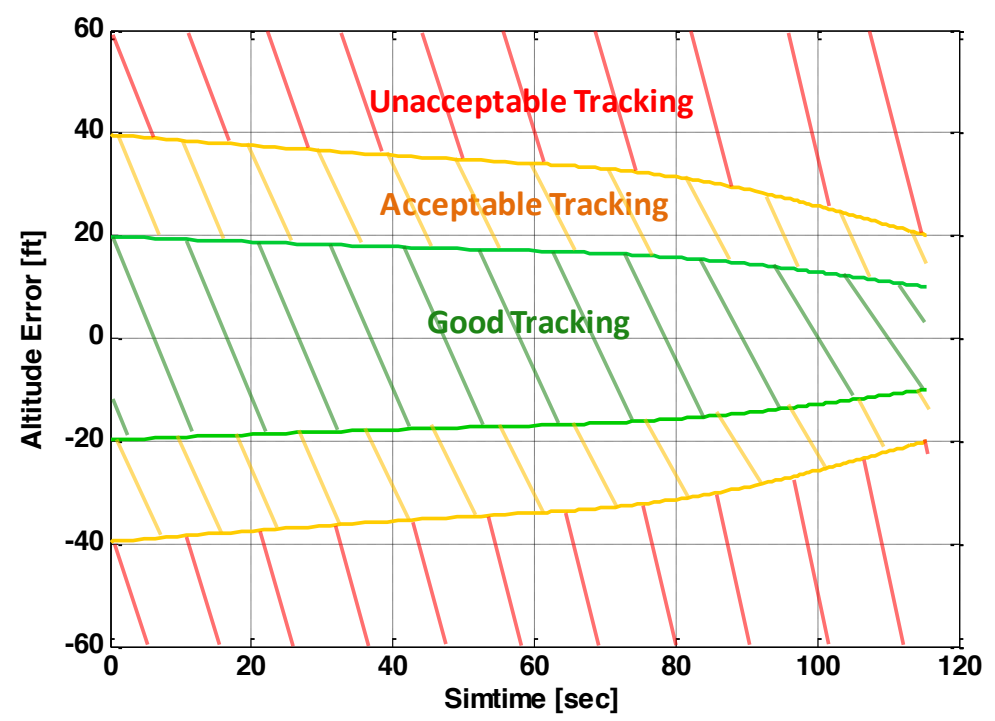

Figure 1-29: Altitude error performance metrics. 
Robustness of the controller to aerodynamic uncertainties is evaluated based on whether the controller can track commands within an acceptable tolerance. The robustness is quantified by the amount of uncertainty that can be inserted into the plant until the controller performance degrades and becomes unacceptable. 


\section{Baseline Controller}

The baseline controller is a dynamic inversion and backstepping controller designed without unmodeled plant aerodynamic uncertainties. No aerodynamic uncertainties implies that aerodynamic tables used for the controller is the same set of tables used for the plant model. The following sections will describe formulations for a dynamic inversion and backstepping controller and its implementation into a Simulink model.

\subsection{Dynamic Inversion Controller}

\subsubsection{Motivation}

RLVs deal with highly nonlinear dynamics across a wide flight envelope so a controller must be able to provide inner loop stability throughout its flight. Dynamic inversion is a nonlinear control method that can be used to augment the vehicle stability. It eliminates the need for a trajectory dependent gain scheduling since it is based on a full envelope design. This requires only one controller to be used for a full envelope flight. A dynamic inversion process "cancels" natural vehicle dynamics with its inner loop feedback. The inverted dynamics are replaced by desired linear dynamics with an outer loop feedback on body rate.

\subsubsection{Control Law Formulation}

The objective of a dynamic inversion controller is to track body rate commands (PQR) by specifying body acceleration rates. Dynamic inversion transforms a nonlinear system into a decoupled linear system through feedback. The transformation allows a simple controller to be

designed to give the system desired closed loop dynamics ${ }^{1}$. The controller tracks desired 
dynamics by essentially cancelling wing-body moments with moments produced by control effectors ${ }^{13}$.

A vehicle's rotation dynamics in matrix form are expressed as Equation (2-1), assuming the time derivative of an inertia matrix is zero since mass is constant during approach and landing.

$$
\dot{\omega}=I^{-1}(M-\omega \times I \omega)
$$

Where $\dot{\omega}$ is body rate acceleration vector, $\mathrm{M}$ is the body axis moment vector, $\mathrm{L}, \mathrm{M}$, and N, $\omega$ is the body rate vector, $\mathrm{P}, \mathrm{Q}$, and $\mathrm{R}$, and $I$ is the inertia matrix in slug- $\mathrm{ft}^{2}$. Equation (2-1) can be rewritten in the form ${ }^{16}$ :

$$
\dot{\omega}=f(\omega, P)+g(P) \delta
$$

Where $P$ represents state variables like $\alpha$, Mach, and $\beta$ that affect moments acting on the vehicle and $\omega$ are the body rates. $f(\omega, P)$ represents nonlinear dynamics and accelerations due to moments produced from a bare airframe. The $g(P) \delta$ term represents the acceleration contribution from moments generated by control surface deflections, and $g(P)$ is a control effectiveness term. $\delta$ is the control deflection assumed to be a $3 \times 1$ matrix in order for the matrix math to work. Control effectiveness is how body accelerations change with control deflection. This is computed by ${ }^{16}$ :

$$
g(P)=I^{-1}\left[\begin{array}{lll}
\frac{d C l}{d \delta_{1}} & \frac{d C l}{d \delta_{2}} & \frac{d C l}{d \delta_{3}} \\
\frac{d C m}{d \delta_{1}} & \frac{d C m}{d \delta_{2}} & \frac{d C m}{d \delta_{3}} \\
\frac{d C n}{d \delta_{1}} & \frac{d C n}{d \delta_{2}} & \frac{d C n}{d \delta_{3}}
\end{array}\right]
$$


The control law is attained by solving for $\delta$ from Equation (2-2) by subtracting $f(\omega, P)$ and inverting $g(P)$ as seen in Equation (2-4) ${ }^{16}$. This equation represents nonlinear plant dynamic cancellation.

$$
\delta=g^{-1}(P)[\dot{\omega}-f(\omega, P)]
$$

Commanded angular body rates: $\mathrm{P}, \mathrm{Q}$, and R, are specified implicitly through desired control variables, $\dot{\omega}_{\text {des }}$. Subsituting $\dot{\omega}$ with $\dot{\omega}_{\text {des }}$ gives the dynamic inversion control law shown as Equation $(2-5)^{16}$

$$
\delta=g^{-1}(P)\left[\dot{\omega}_{d e s}-f(\omega, P)\right]
$$

The control law operates on desired acceleration terms, $\dot{\omega}_{\text {des }}$, so a mapping function is created between angular rate commands, $\omega_{c m d}$, and $\dot{\omega}_{d e s}$, called desired dynamics or a prefilter. The mapping function is an outer loop that replaces nonlinear dynamics with a preferred set of desired linear dynamics. A prefilter achieves desired close loop body rate response by operating on errors between commanded and actual angular rates. Any combination of proportional dynamics, proportional-integral (PI) dynamics, proportional-derivative (PD) dynamics, or PID dynamics can be used as desired dynamics. Prefilter options will be explained in Section 2.1.5. Figure 2-1 depicts the dynamic inversion control law.

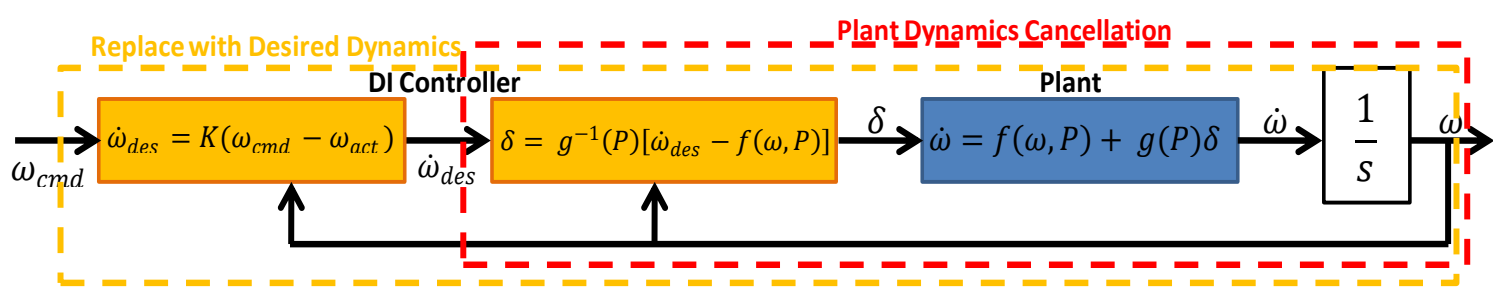

Figure 2-1: The dynamic inversion control law depiction. 


\subsubsection{Lyapunov Stability}

For a system of the form:

$$
\dot{x}=f(x, u)
$$

With an equilibrium states at:

$$
f(0,0)=0
$$

And with a feedback control law:

$$
u=\alpha(x)
$$

The origin of the system,

$$
\dot{x}=f(x, \alpha(x))
$$

is globally asymptotically stable (GAS) if there exists a Lyapunov function that satisfies:

$$
\begin{gathered}
V(x) \geq 0 \\
\dot{V}=\frac{d V}{d x} f(x, \alpha(u)) \leq-W(x) \leq 0
\end{gathered}
$$

Rotational dynamics of the RLV plant model from Equation (2-2) can be described as Equation (2-12).

$$
\dot{x}=f(\omega, P)+g(P) u
$$

The dynamic inversion control law from Equation (2-4) can be rewritten in the form:

$$
u=g^{-1}(P)[-k x-f(x, P)]
$$


The prefilter computes desired body accelerations by operating on body rate errors, which requires feeding back body rates, represented by $-k x$. The CLF in Equation (2-14) is chosen to represent the system above.

$$
V(x)=\frac{1}{2} x^{2}
$$

The derivative of the CLF along state, $\mathrm{x}$, becomes:

$$
\dot{V}(x)=x \dot{x}
$$

Substituting Equation (2-12) into $\dot{x}$ in Equation (2-15) above:

$$
\dot{V}(x)=x(f(x, P)+g(P) u)
$$

Then substituting the dynamic inversion control law from Equation (2-13) for $u$ in Equation (2-16) gives:

$$
\begin{gathered}
\dot{V}(x)=x\left[f(x, P)+g(P)\left(g^{-1}(P)[-k x-f(x, P)]\right)\right] \\
\dot{V}(x)=x[f(x, P)-k x-f(x, P)] \\
\dot{V}(x)=-k x^{2}
\end{gathered}
$$

The CLF is negative definite as long as:

$$
\dot{V}(x) \leq 0 \text { for } k>0
$$

A system is GAS when a CLF is negative definite as in the condition represented by Equation $(2-20)$. This equation is satisfied as long as gain, $k$, used in the prefilter on body rate errors are positive. 
Several assumptions are made to help simplify the Lyapunov stability formulation that does not describe the system exactly. This may affect robustness of the controller when it is implemented into a $6 \mathrm{DoF}$ simulation, even if a system is GAS using the proof above. A CLF is only created to represent body rate states in the rotational dynamics, but a 6 DoF simulation has acceleration, position, and velocity states that are not represented. Moreover, the control law formulation used to prove stability of the system assumes control surface contribution to body acceleration can be linearly represented as $g(P, u)$ in Equation (2-12). A linear representation means that change in moment with respect to change in control deflection is constant. However, the RLV control surface aerodynamics are nonlinear so control effectiveness varies with control deflection. Body acceleration contribution should be represented as $g(P, u)$ if the system is accurately portrayed. The control formulation also assumes that the control effectiveness matrix, $g(P)$, can be inverted to solve for $u$. This may not always be the case if $g(P)$ is close to singular or if a solution doesn't exist without exceeding control deflection limits. All of the assumptions above simplifies the system when selecting a CLF so the dynamic inversion controller may not possess robustness characteristics that are normally associated with the definition of a GAS.

\subsubsection{Control Allocation}

The dynamic inversion control law derived in Section 2.1.2 assumes the number of control effectors equals the number of controlled variables; however, the RLV has seven control surfaces. Moreover, the control law assumes body accelerations are linear in controls in order to solve for control deflections required to produce desired accelerations. RLV control effectors are nonlinear, meaning that control derivatives change with control deflections. Each control effectors are also capable of producing moments in more than one axis. This requires a method to allocate control to all of the control surfaces. Therefore, a control allocation algorithm needs to be 
augmented with a dynamic inversion control law to handle these issues. The control allocation scheme still uses a linear approximation of control deflection effectiveness, but introduces an intercept term to improve accuracy from assuming linearity ${ }^{15}$.

Figure 2-2 illustrates a two-dimensional example of how a local approximation of a control surface curve with an intercept term is an improved method over a linear global slope approximation. $\mathrm{G}_{\mathrm{o}}(\mathrm{P}, \delta)$ represents a moment value at a particular flight condition, $\mathrm{P}$, and a deflection, $\delta$.

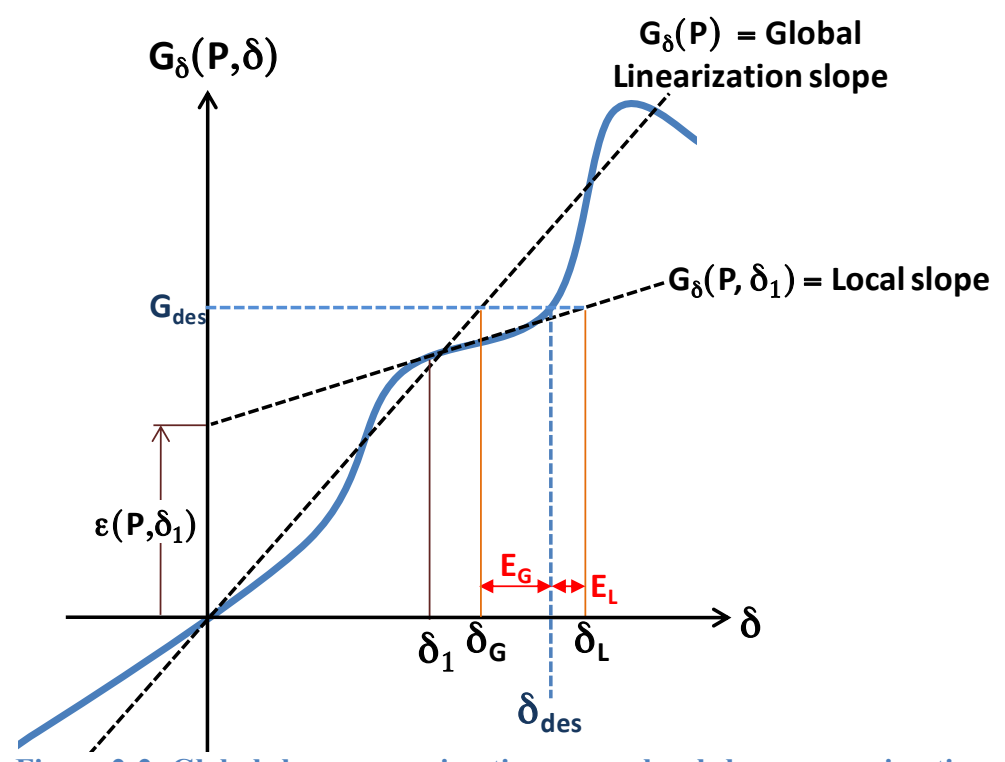

Figure 2-2: Global slope approximation versus local slope approximation.

Slope $G_{\circ}(P)$ represents a global approximation of the control effectiveness, which is the change in moment due to change in control surface deflection. Slope $G_{o}\left(P, \delta_{1}\right)$ is the local approximation at the current operating point, $\mathrm{P}$ and $\delta_{1}$, with $\varepsilon\left(\mathrm{P}, \delta_{1}\right)$ corresponding to an intercept term. An intercept term is a value adjusted to control commands for using a local approximation to compute a moment at a particular flight condition, represented by $\left(\mathrm{P}, \delta_{1}\right)^{12}$. The term $\delta_{\mathrm{L}}$ depicts a control surface deflection computed if $\mathrm{G}_{0}\left(\mathrm{P}, \delta_{1}\right)$, a local slope, is used as the control derivative to compute a deflection needed to achieve a desired moment, $G_{\text {des. }} . \delta_{G}$ is a control surface deflection 
calculated to get a desired moment, using the global linearized slope. $\mathrm{E}_{\mathrm{L}}$ represents deflection error from localizing the control derivative, while $\mathrm{E}_{\mathrm{G}}$ represents the error from using the global linearized slope. The error produced from localizing the control derivative is much smaller in comparison proving that an intercept term is a better linear approximation to use in a control allocation scheme.

To implement a linear control allocator with dynamic inversion, moments from Equation (2-1) is separated into $G_{B A E}$ and $G_{s}$, where $G_{B A E}$ are moments produced from a bare airframe and $\mathrm{G}_{\mathrm{s}}$ are sum of moments produced by control surfaces. The dynamic equation in Equation (2-1) can now be rewritten as Equation $(2-21)^{13}$.

$$
\begin{gathered}
\dot{\omega}=I^{-1}\left(G_{B}-\omega \times I \omega\right) \\
\text { where } G_{B}=G_{B A E}(\omega, P)+G_{\delta}(P, \delta)=\left[\begin{array}{c}
L \\
M \\
N
\end{array}\right]_{B A E}+\left[\begin{array}{c}
L \\
M \\
N
\end{array}\right]_{\delta}
\end{gathered}
$$

In order to put the above equation in a similar form as Equation (2-2), control dependent body acceleration and bare airframe dependent body acceleration terms are separated ${ }^{13}$ :

$$
\begin{array}{cl}
\dot{\omega}=f(\omega, P)+g(P, \delta) \\
\text { where } & f(\omega, P)=I^{-1}\left(G_{B A E}-\omega \times I \omega\right) \\
& g(P, \delta)=I^{-1} G_{\delta}(P, \delta)
\end{array}
$$

$f(\omega, P)$ represents accelerations due to a bare airframe, and $g(P, \delta)$ are control dependent accelerations. Control dependent terms has to be linear in controls, $\delta$, in order to use a linear control allocator. The concept of localizing the control derivatives shown in Figure 2-2 is applied to linearize $g(P, \delta)$ at a specific flight condition so that Equation (2-24) can be rewritten as Equation $(2-25)^{13}$. 


$$
g(P, \delta)=I^{-1} G_{\delta}(P) \delta+I^{-1} \varepsilon(P, \delta)
$$

Where $\varepsilon(P, \delta)$ is the intercept term and $G_{\delta}(P)^{13}$ is populated by taking the local slopes of control moment curves at a current operating point:

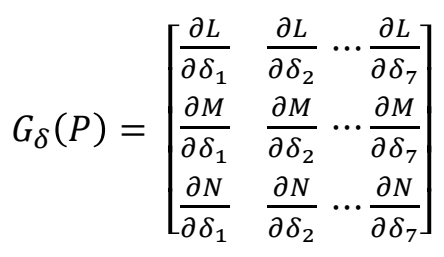

Acceleration contributions from intercept terms are derived by substituting Equation (2-24) into $g(P, \delta)$ from Equation (2-25) and solving for $\varepsilon(P, \delta)^{12}$.

$$
\begin{gathered}
I^{-1} G_{\delta}(P, \delta)=I^{-1} G_{\delta}(P) \delta+I^{-1} \varepsilon(P, \delta) \\
I^{-1} \varepsilon(P, \delta)=I^{-1} G_{\delta}(P, \delta)-I^{-1} G_{\delta}(P) \delta \\
\text { where } I^{-1} G_{\delta}(P, \delta)=I^{-1}\left[\begin{array}{c}
L \\
M \\
N
\end{array}\right]_{(P, \delta)}
\end{gathered}
$$

Where $G_{\delta}(P, \delta)$ is the total moment caused by control surface deflections and $G_{\delta}(P)$ is the local slope of the control effectiveness slope from Equation (2-26).

To formulate the control law with a linear allocator, dynamics from Equation (2-22) is reformulated by substituting Equation $(2-25)$ into $g(P, \delta)$.

$$
\dot{\omega}=f(\omega, P)+I^{-1} G_{\delta}(P) \delta+I^{-1} \varepsilon(P, \delta)
$$

Desired acceleration is substituted into the body acceleration term in Equation (2-29):

$$
\dot{\omega}_{d e s}=f(\omega, P)+I^{-1} G_{\delta}(P) \delta+I^{-1} \varepsilon(P, \delta)
$$


Then a linear approximation of control dependent acceleration is determined using Equation $(2-30)^{13}$

$$
\begin{aligned}
& I^{-1} G_{\delta}(P) \delta=\dot{\omega}_{\text {des }}-f(\omega, P)-I^{-1} \varepsilon(P, \delta) \\
& I^{-1} G_{\delta}(P) \delta=B \delta \\
& I^{-1} G_{\delta}(P) \delta=d_{\text {des }}
\end{aligned}
$$

$B$ is the control derivative of body accelerations with respect to control surface deflections. The term, $d_{d e s}$, represents the difference between the desired acceleration, $\dot{\omega}_{d e s}$ and acceleration induced by current flight condition, $f(\omega, P)-I^{-1} \varepsilon(P, \delta)$. This difference is the amount of acceleration that needs to be compensated by control surfaces. Therefore, the control allocator objective is to find proper $\delta$ s to make:

$$
B \delta=d_{d e s}
$$

The pseudoinverse solution can be used to solve Equation (2-34). The pseudoinverse of B, shown in Equation (2-35), provides a solution for an underdetermined system where the amount of control surfaces exceeds the number of control variables ${ }^{5}$. The solution below becomes the control allocation equation.

$$
\delta_{P}=-c+B^{T}\left(B B^{T}\right)^{-1}\left[d_{d e s}-B c\right]
$$

Where $\delta_{P}$ is preferred control surface deflections required to produce accelerations equal to $\mathrm{d}_{\text {des }}$. The c term is an offset vector representing control surface deflections where local slope approximations are taken, and $\mathrm{Bc}$ is accelerations produced by the offset vector, $\mathrm{c}$. Although the 
pseudoinverse solution will not be unique, it provides a least squares solution, shown in Equation (2-36), as long as adequate control authority exists ${ }^{5}$.

$$
\min _{x}\|A x-b\|
$$

\subsubsection{Prefilter}

Dynamic inversion takes desired body acceleration as an input, rather than body rates commands. A prefilter provides a way to transform body rate commands into desired body accelerations. The simplest way to design a prefilter is to use a proportional gain on body rate error as shown in Figure 2-3.

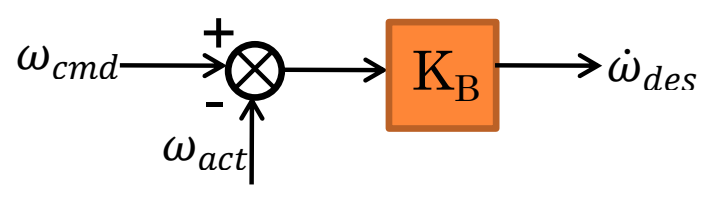

Figure 2-3: Proportional gain used as the prefilter.

A closed loop system of a prefilter from the figure above can be written in transfer function form as:

$$
\frac{\omega_{a c t}}{\omega_{c m d}}=\frac{\frac{1}{2} K_{B}}{s+\frac{1}{2} K_{B}}
$$

Proportional feedback provides a nice first order closed loop response with the bandwidth of a system set at $\mathrm{K}_{\mathrm{B}}{ }^{15}$. This closed loop response can only be achieved if natural vehicle dynamics are cancelled exactly by a dynamic inversion controller. The dynamics will not be cancelled precisely if control surfaces saturate or if there are modeling errors. 
Another option to compensate for modeling errors is to introduce an integral term in a prefilter structure shown in Figure 2-4.

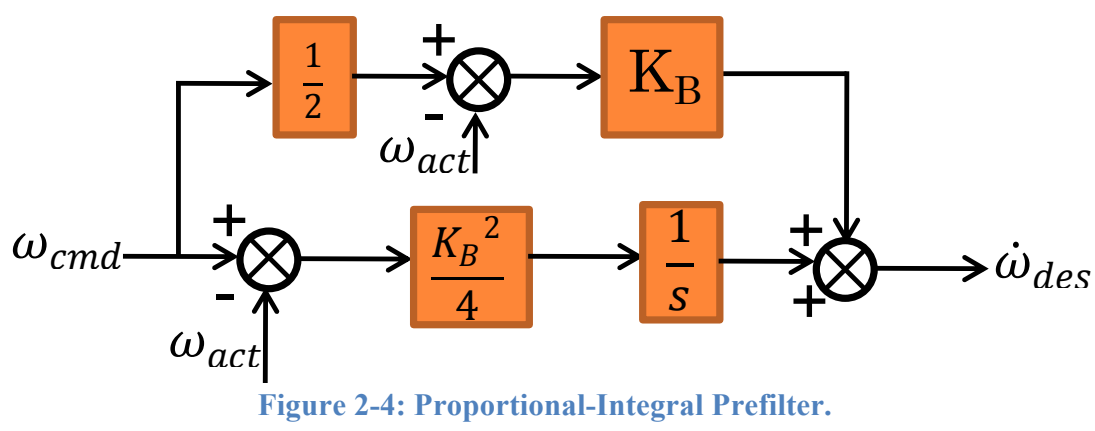

An integral action will close errors caused by modeling uncertainties, while a feedforward term will compensate for lag introduced by an integral by adding phase margin at crossover ${ }^{15}$. The closed loop transfer function is written in the same form as the pure proportional feedback from Equation (2-37) because of a stable pole-zero cancellation ${ }^{15}$. An advantage of using this prefilter structure is that it still provides a first order closed loop response as long as there are no inversion errors.

\subsubsection{Dynamic Inversion Controller Implementation into Simulink}

Figure 2-5 depicts the dynamic inversion control law formulated in Sections 2.1.4 and 2.1.5 inside the Simulink model. 


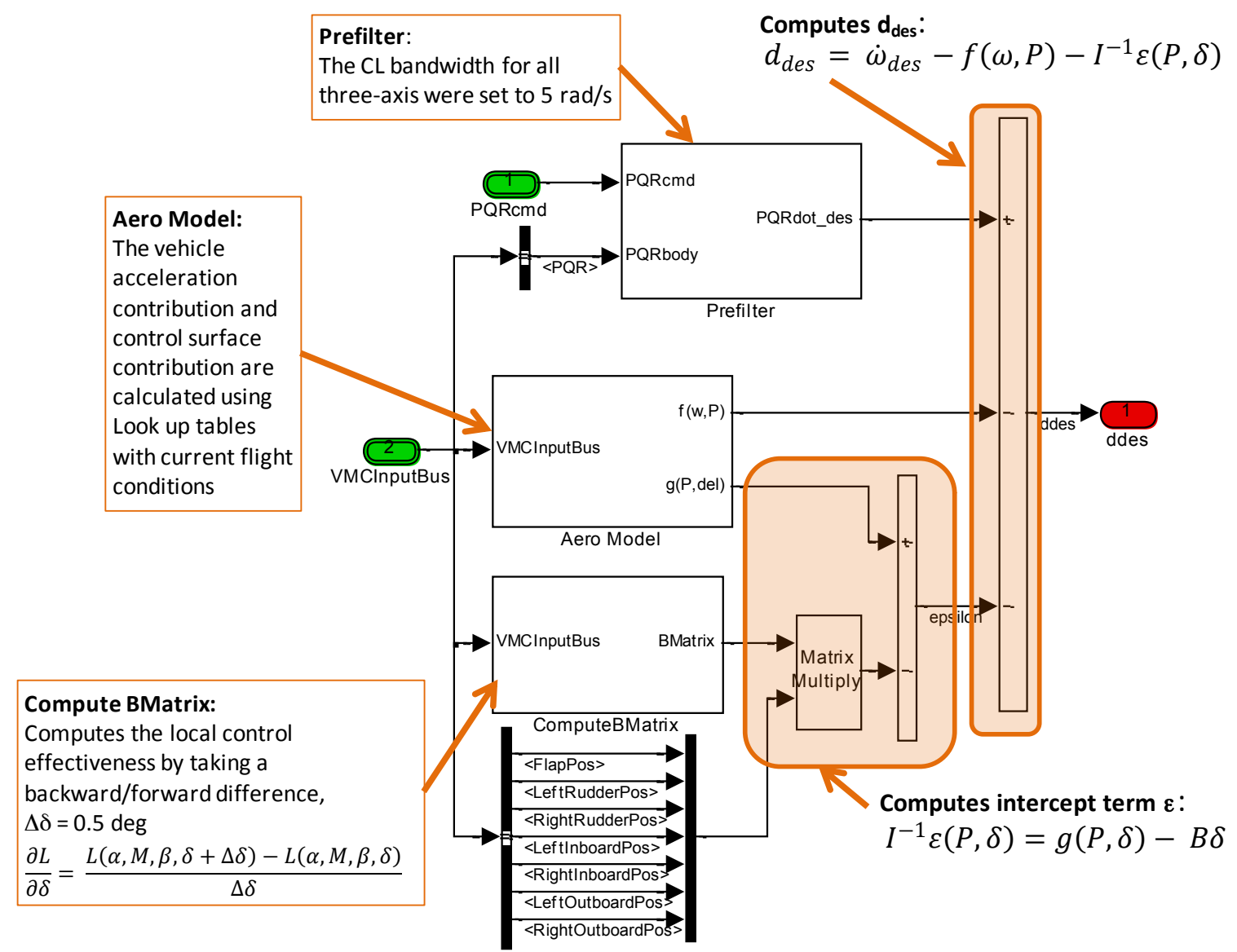

Figure 2-5: Dynamic Inversion control Law Implementation into Simulink.

The prefilter structure is chosen as proportional-integral (PI) feedback shown in Figure 2-4. Bandwidth of the inner loop was set to be $5 \mathrm{rad} / \mathrm{sec}$, so $\mathrm{K}_{\mathrm{B}}$ from Figure 2-4 was equal to 5 . Both types of prefilter structures, proportional and PI, were tested during a validation process to make sure the dynamic inversion controller was implemented correctly. Each prefilter was tested with aerodynamic modeling error before selecting a structure showing the best performance. The next section, 2.1.7, will show results from this comparison and why a PI prefilter was chosen.

The "Aero Model" block from Figure 2-5 computes $f(\omega, P)$, body acceleration contribution from the wing-body, and $g(P, \delta)$, the total body acceleration contribution from 
control surfaces. $f(\omega, P)$ is computed from Equation (2-23), and $g(P, \delta)$ is computed from Equation (2-24). Figure 2-6 shows how these equations are implemented into a Simulink model.

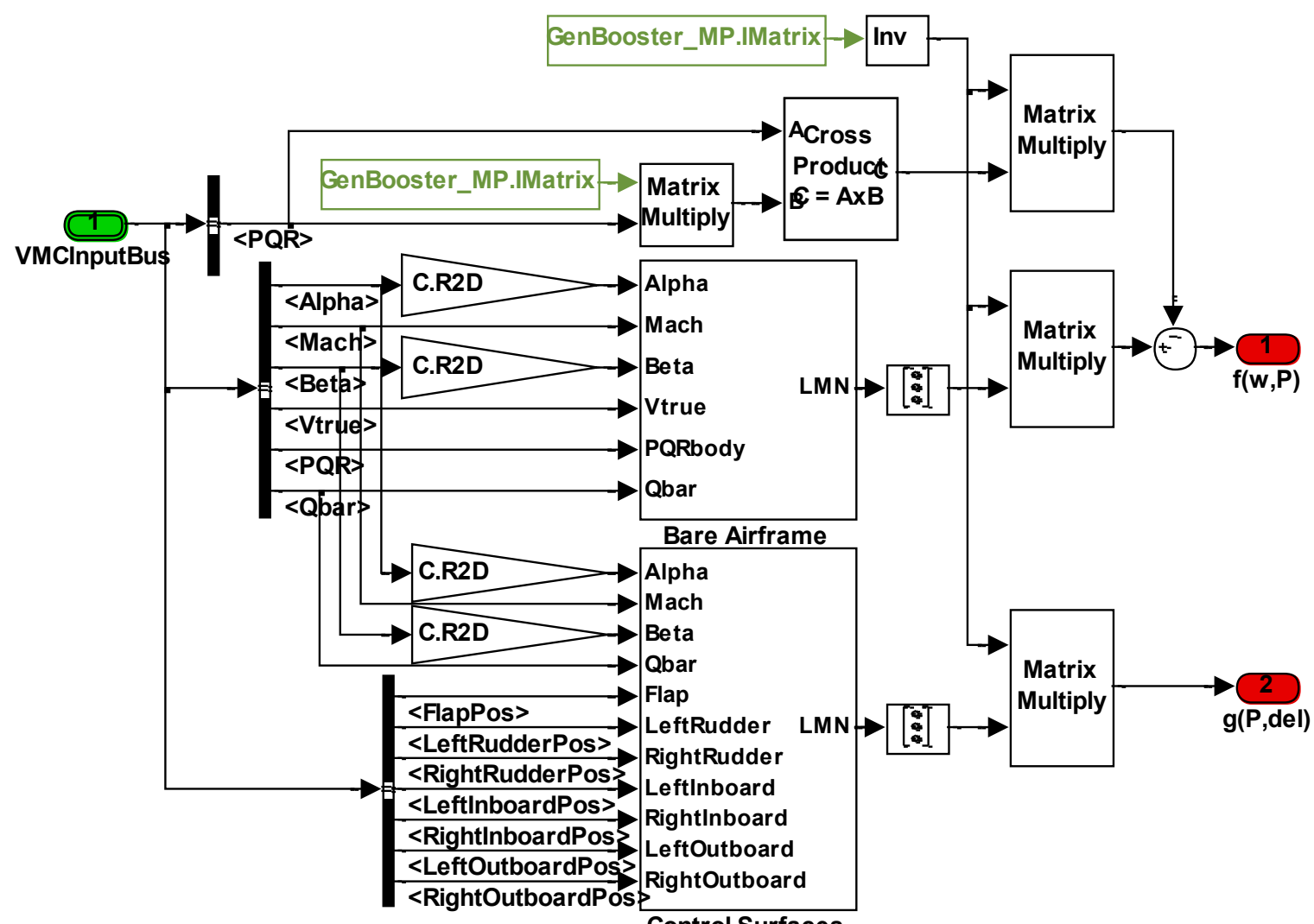

Figure 2-6: Inside the "Aero Model" block to compute $f(\omega, P)$ and $g(P, \delta)$.

Moments computed inside the "Bare Airframe" and the "Control Surfaces" block in the figure above contains the same functions inside the Plant model computing the vehicle's moment in Figure 1-13. Control effectiveness is computed inside the "ComputeBMatrix" block in Figure 2-5. Control allocation requires control derivatives are computed locally. Local derivatives are calculated by taking a forward difference or backward difference with respect to changes in control surface deflections at a current operating point. Figure 2-7 depicts how control effectiveness is found for the flap inside a Simulink model. 


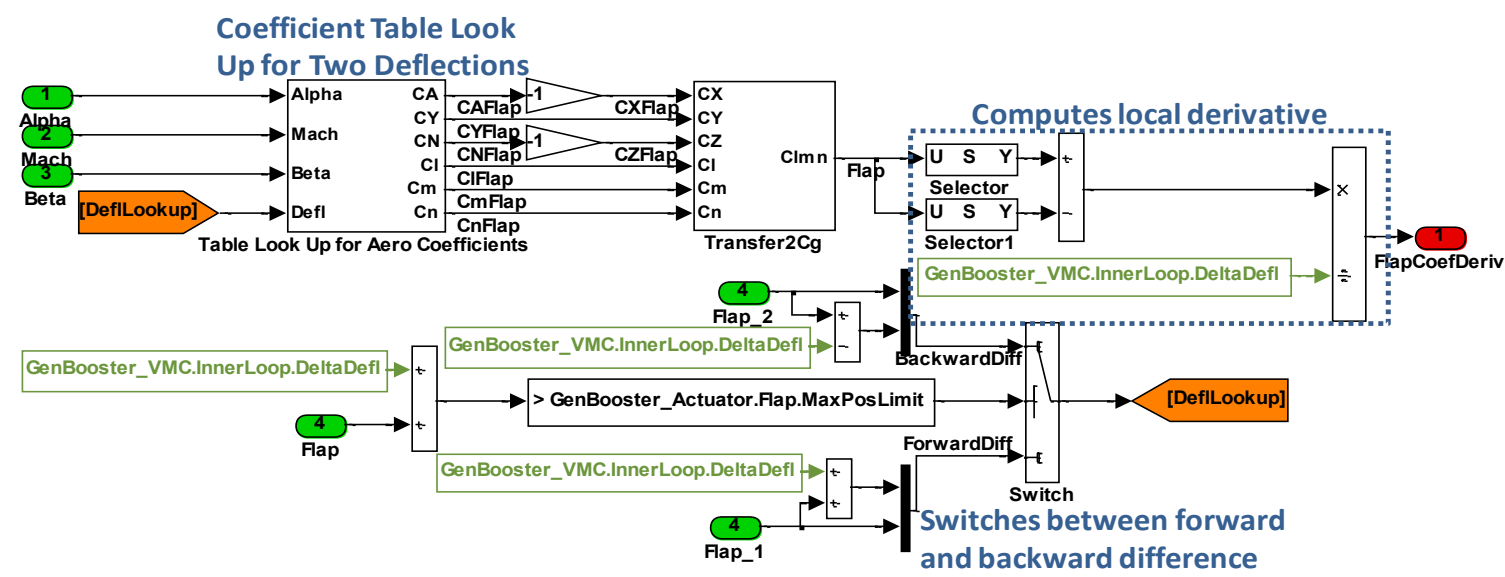

Figure 2-7: Computing the control effectiveness for the Flap.

Deflection step used to find a local control derivative is set to 0.5 degrees. If the sum of a current deflection position and the deflection step exceeds a maximum deflection value, then a backward difference is used to find the local slope, otherwise a forward difference is used. The table look up blocks find moment and force coefficients at a current flight condition and for two control surface deflections needed to calculate the local slope. Coefficients are transferred to the vehicle's CG before a forward or backward difference is computed. Control derivatives are in coefficient form so they will have to be transformed into moment derivatives. This is repeated for each control surface to build up a $3 \times 7$ matrix used in Equation (2-26).

Once control effectiveness and desired accelerations are computed, signals feed into the control allocator, depicted in Figure 2-8. Equation (2-35) is the control allocation algorithm implemented into Simulink. 


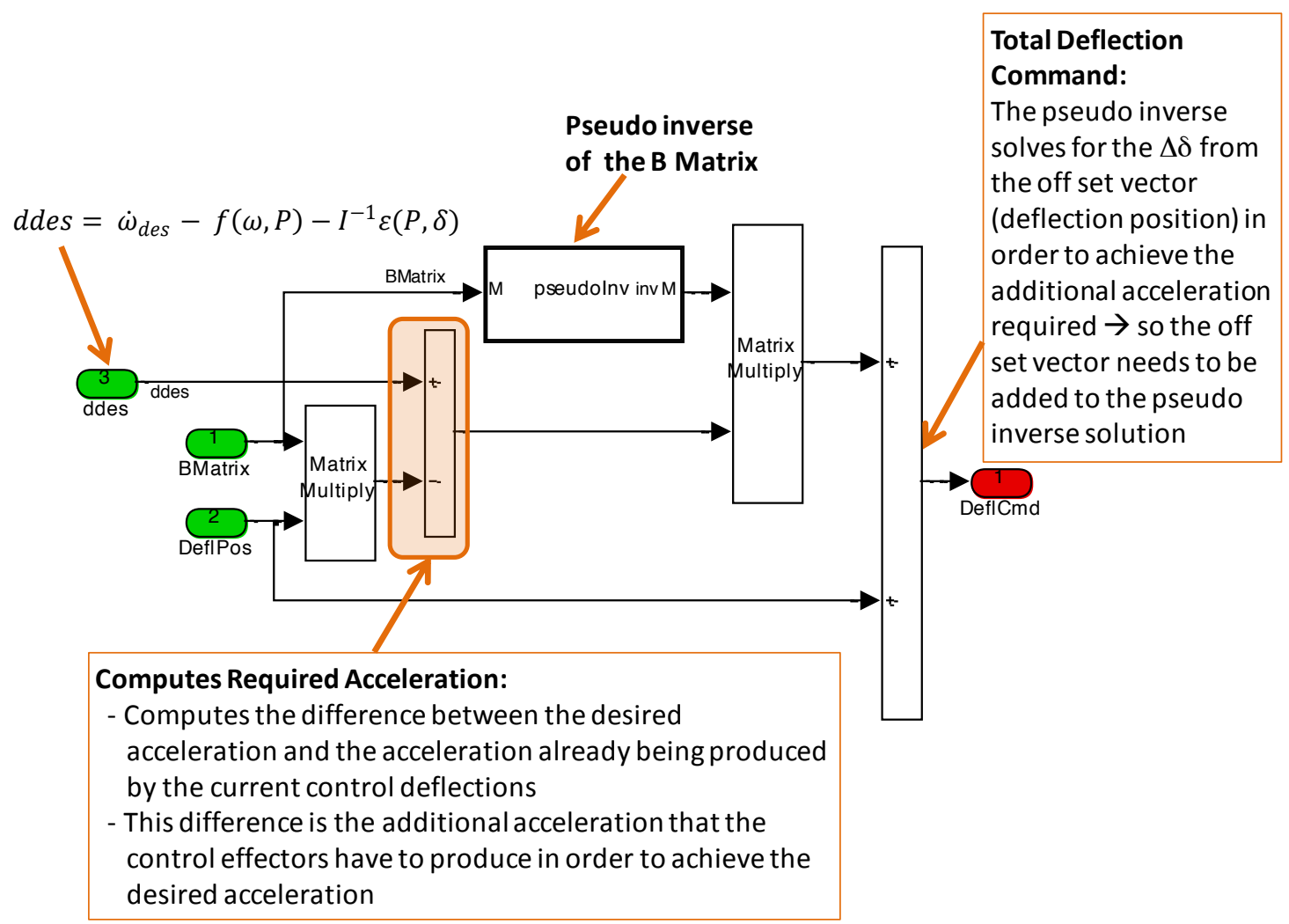

Figure 2-8: The control allocator to compute the control surface deflection commands.

\subsubsection{Dynamic Inversion Validation}

The dynamic inversion controller has to be tested within a simulation environment to verify that the algorithm is implemented correctly and performs within figures of merit specified in Section 1.10. PQR doublet test cases, described in Section 1.9.2.1, are conducted for validation, assuming no modeling errors between vehicle aerodynamics in the controller and in the plant model. Performance achieved from the controller will be set as the baseline to compare all other controller performances containing modeling error. However, a prefilter structure has to be selected first before evaluating a baseline controller. 


\subsubsection{Prefilter Selection}

There are two choices available for the prefilter: the proportional rate feedback, or the proportional-integral (PI) feedback, as explained in Section 2.1.5. They both exhibit first order closed loop performance if dynamic inversion can invert plant dynamics without saturating control surfaces or without aerodynamic modeling errors. However, the objective of this thesis is to test robustness of the controller to aerodynamic modeling error, so the prefilter that exhibits the best performance under those conditions should be selected. Both prefilters were tested with $+/-30 \%$ aerodynamic modeling error for static and control surface aerodynamics. Controller performances are compared for tracking body rate doublets using test cases described in Section 1.9.2.1. The results are shown in Figure 2-9 through Figure 2-11.

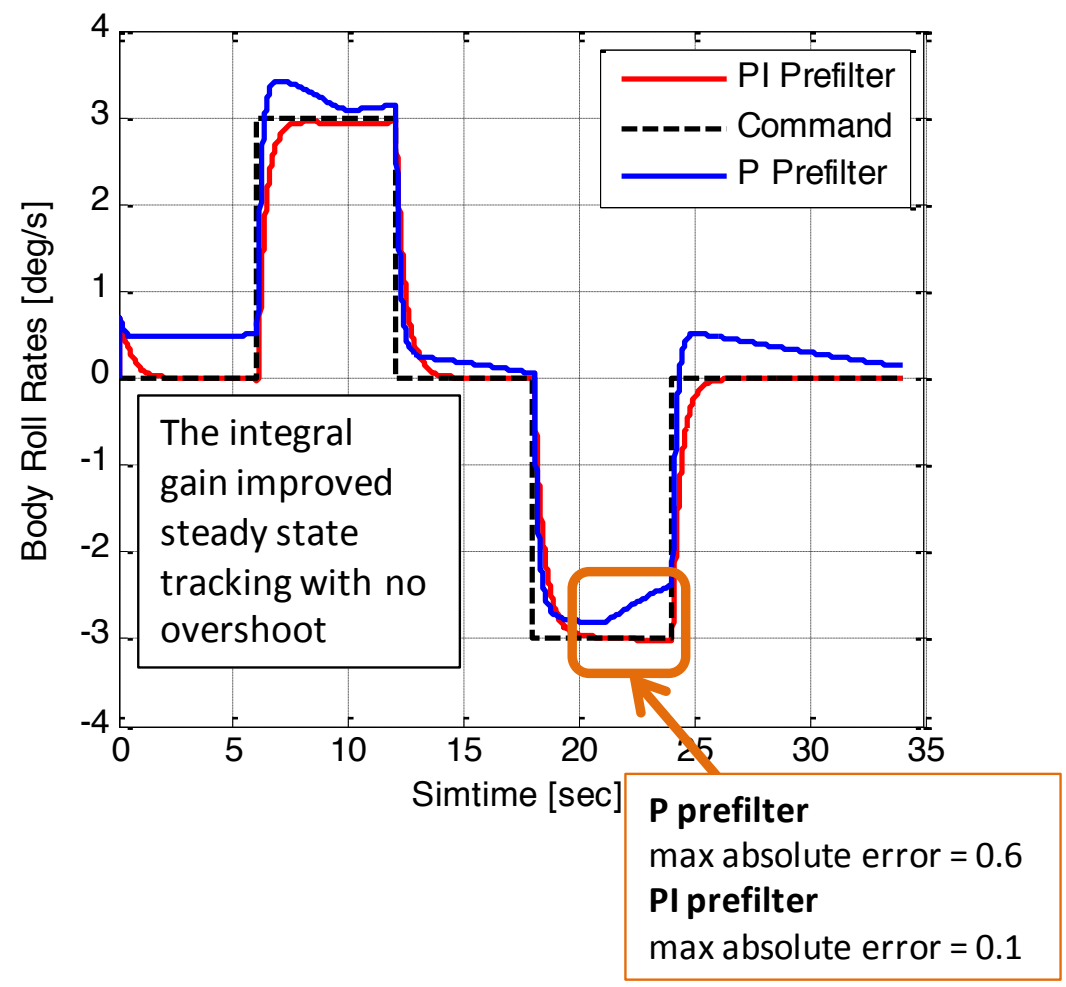

Figure 2-9: Prefilter comparison pitch rate doublet response. 


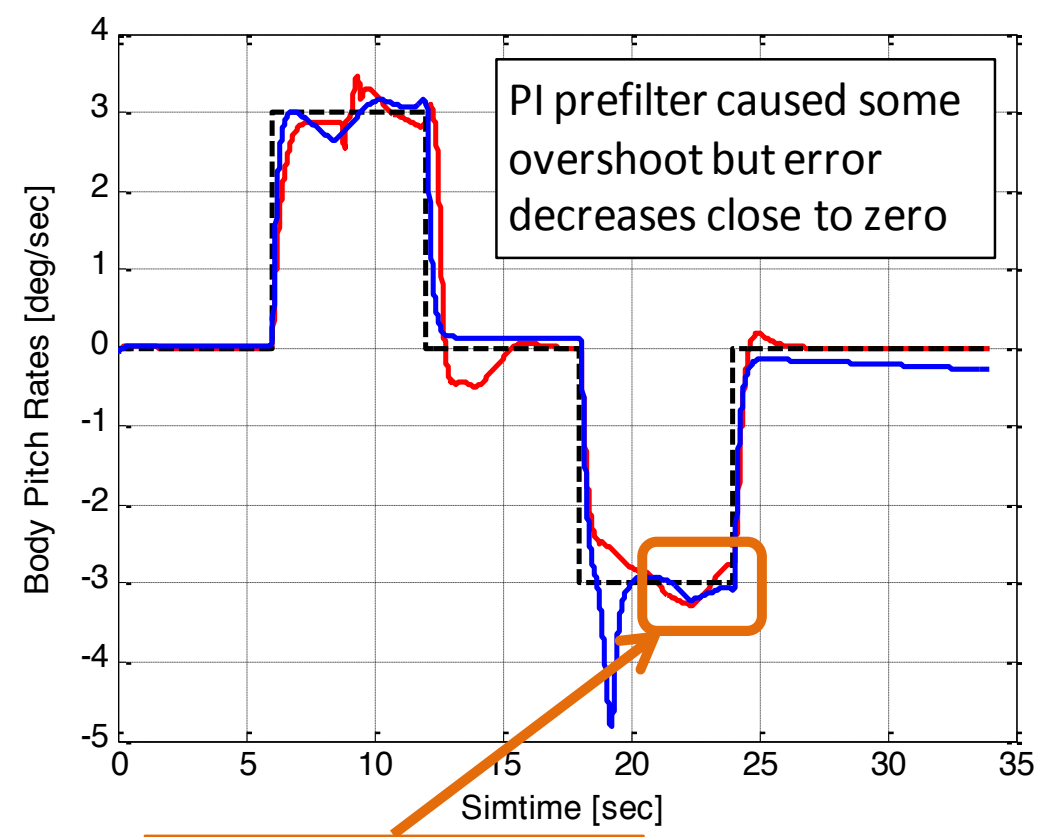

P prefilter

max absolute error $=0.12$

PI prefilter

max absolute error $=0.05$

Figure 2-10: Prefilter comparison for roll rate doublet response.

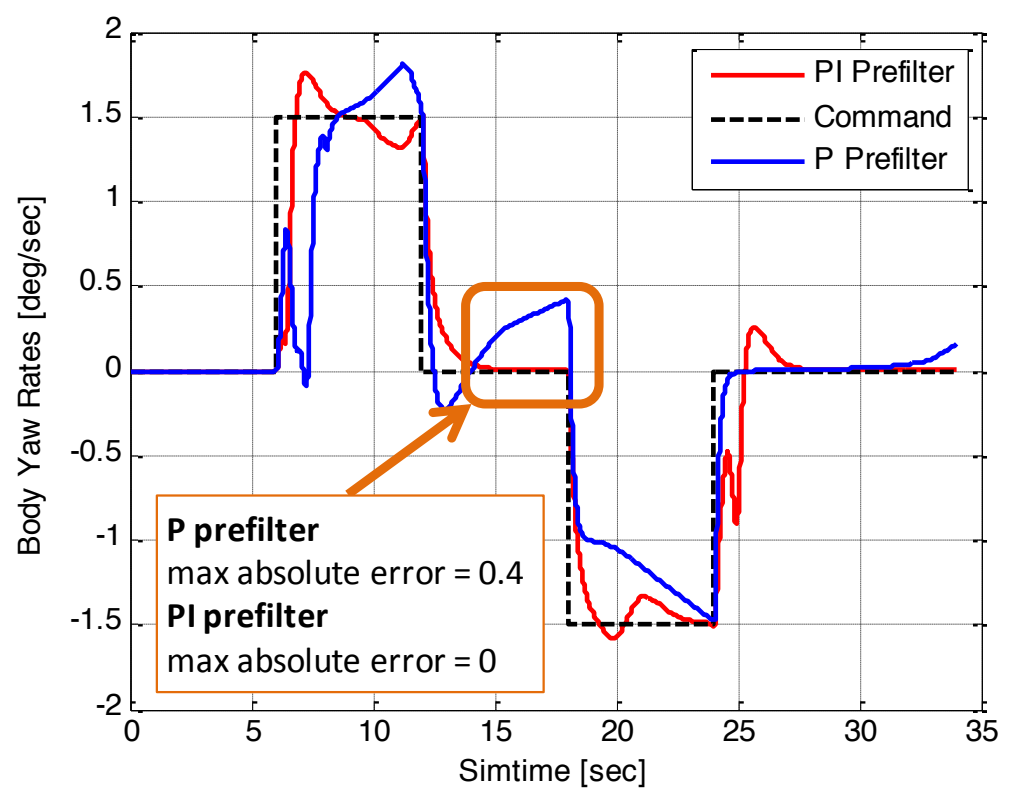

Figure 2-11: Prefilter comparison for a yaw rate doublet response. 
All figures show a PI prefilter corrects for aerodynamic modelling error better than a proportional gain. The controller with a PI prefilter has a maximum steady state error from 0 $\mathrm{deg} / \mathrm{sec}$ to $0.1 \mathrm{deg} / \mathrm{sec}$, while a proportional prefilter has a maximum steady state error from 0.12 $\mathrm{deg} / \mathrm{sec}$ to $0.6 \mathrm{deg} / \mathrm{sec}$ for the three doublet tests. Integral feedback reduces steady state error without driving the system unstable. Aerodynamic modelling mismatch between the controller and the plant model at times causes the PI prefilter to overshoot where the proportional prefilter did not because of the error building up in the integral. However, the controller with a PI prefilter has lower absolute steady state error for each step command in a doublet test case. Therefore, a PI prefilter is selected for the baseline controller.

\subsubsection{Baseline Controller Performance}

The baseline controller performance is the dynamic inversion controller performance for a given set of body rate commands without any aerodynamic modeling error between an on-board controller model and the "actual" plant model. The same body rate doublet tests were conducted as described in Section 2.1.5 and the prefilter selection. The results from the roll rate (P) doublets are shown in Figure 2-12.

The baseline controller exhibits good tracking according to figures of merit set in Section 1.10 because maximum absolute steady state error is within $4 \%$. Responses for negative step inputs are a nice first order response, but positive step inputs causes the vehicle to initially saturate its control surfaces at 6 seconds and again at 25 seconds before reaching commanded states. Control saturation indicates that Equation (2-32) could not be solved within actuator limits, causing axis saturation. Axis saturation prevents exact plant cancellation, eliminating a positive effect of dynamic inversion that linearizes a plant with feedback. A right-hand plane zero appears when inherent plant dynamics were not cancelled. Zoomed in pictures show that the RLV 
responds inversely to a command when a positive step is commanded after control surfaces saturate. The vehicle response initially goes negative, then positive and ends with zero steady state error. This behavior is usually exhibited by a right hand plane zero. The prefilter integrator state builds up error caused by a right hand plane zero, which results in an overshoot before the vehicle settles on a steady state value.

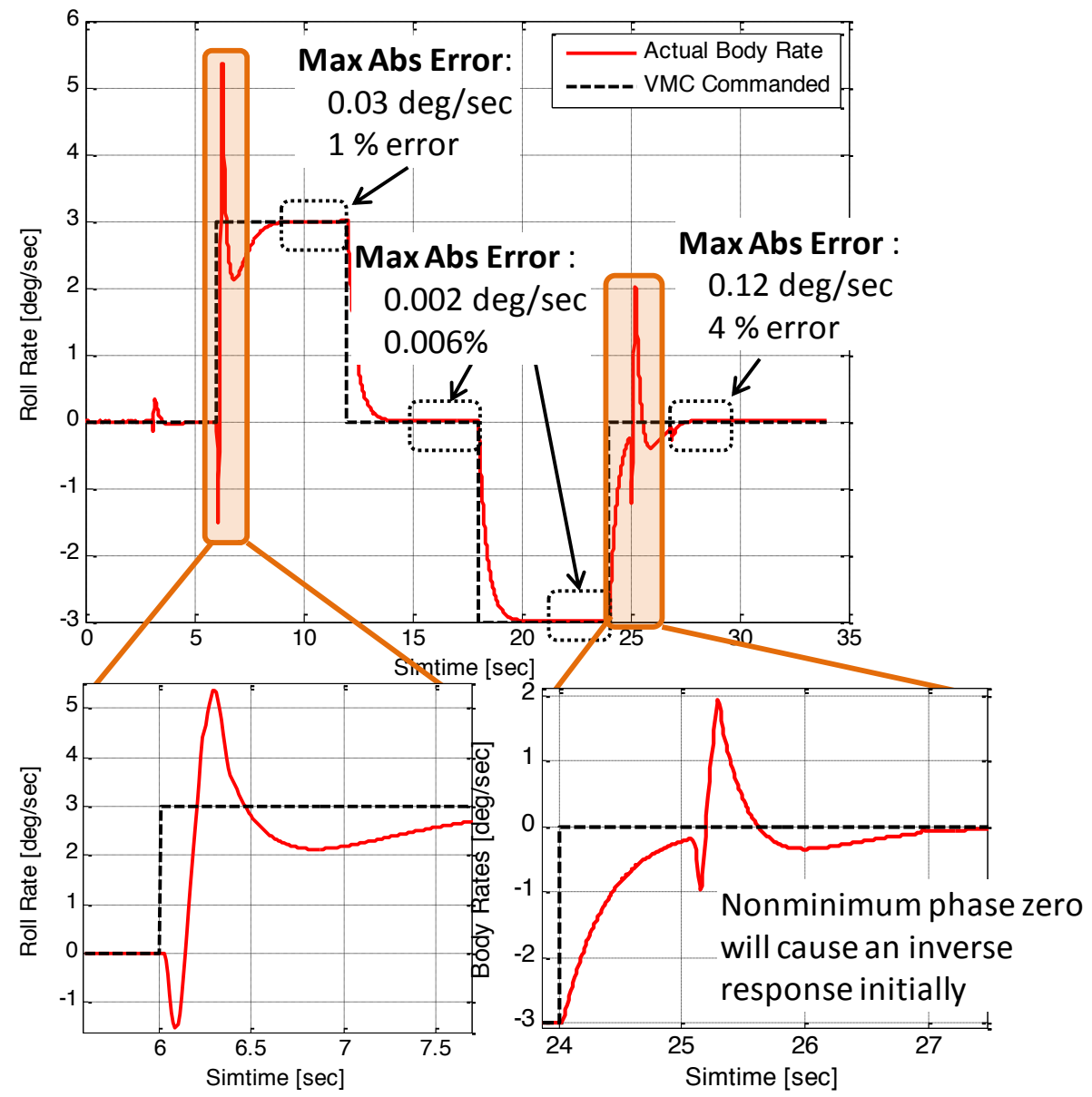

Figure 2-12: Baseline controller performance for roll rate doublet input.

Control saturations occur because the RLV has seven control surfaces, each with limiting amount of control power shared between all three axes. For example, the left inboard control surface can produce roll, pitch, and yaw. If the left inboard control surface is deflects for pitch control, less control authority exists to produce roll and yaw. For a roll doublet test case, no more 
control power is left in the outboard and inboard control surfaces to produce a required roll and pitch acceleration at the same time. The frozen flight condition requires positive pitch acceleration to cancel inherent plant dynamics at the test case condition, as seen in Figure 2-13, in order to maintain zero deg/sec in pitch rate. Figure 2-13 depicts the desired angular acceleration, $d_{d e s}$, fed into the control allocator. The flap will normally provide pitch authority. However, the flap is more effective in producing negative pitching moment rather than positive as seen in Figure 2-14. Therefore, other control surfaces need to be deflected to supplement pitch produced from the flap. Figure 2-14 shows that the right outboard and right inboard surfaces produce the most positive pitching moment due to the length of the moment arms.

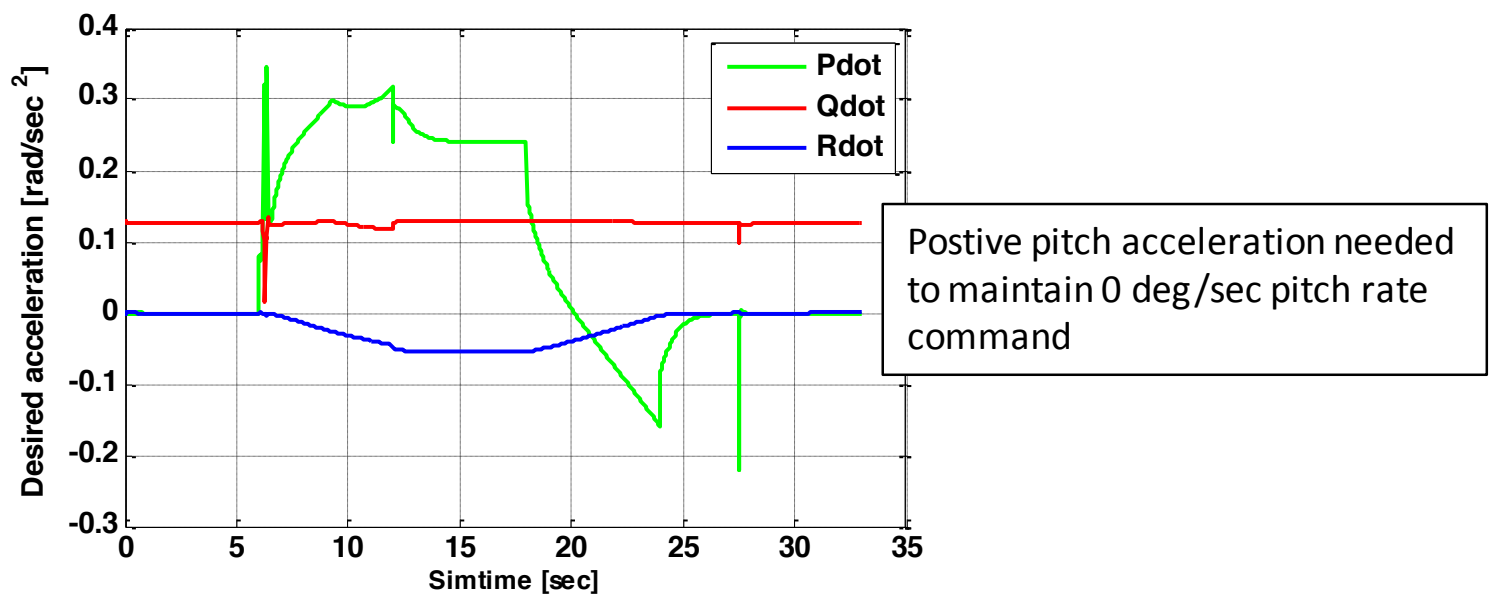

Figure 2-13: Desired acceleration command used by the control allocator.

If these control surfaces provide pitch control, then less control authority exists to provide roll control. Figure 2-14 and Figure 2-15 show control surfaces effectiveness in providing pitch and roll. Like the pitching moment, right outboard and right inboard produce the most positive roll moment. The RLV requires more control power in both axes than control surfaces can provide when positive pitch and positive roll accelerations are needed at the same time, causing Equation (2-32) to solve for control deflections beyond actuator limits. 


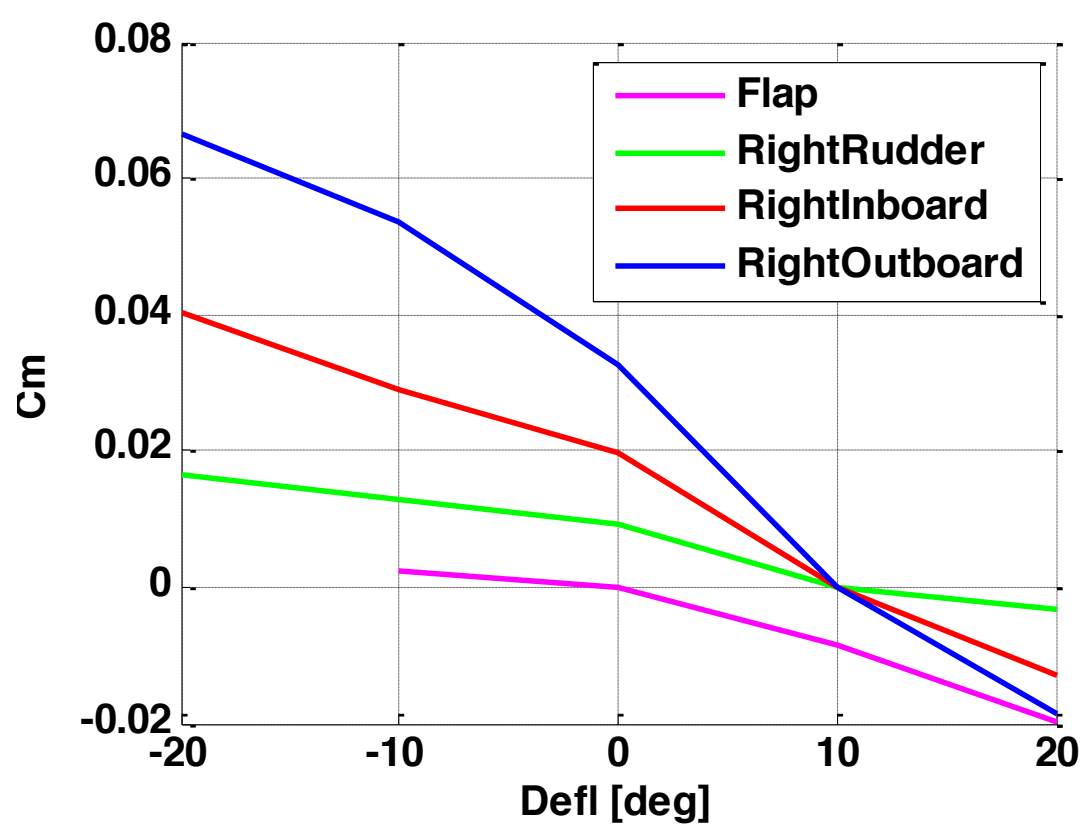

Figure 2-14: Pitch effectiveness for control deflections.

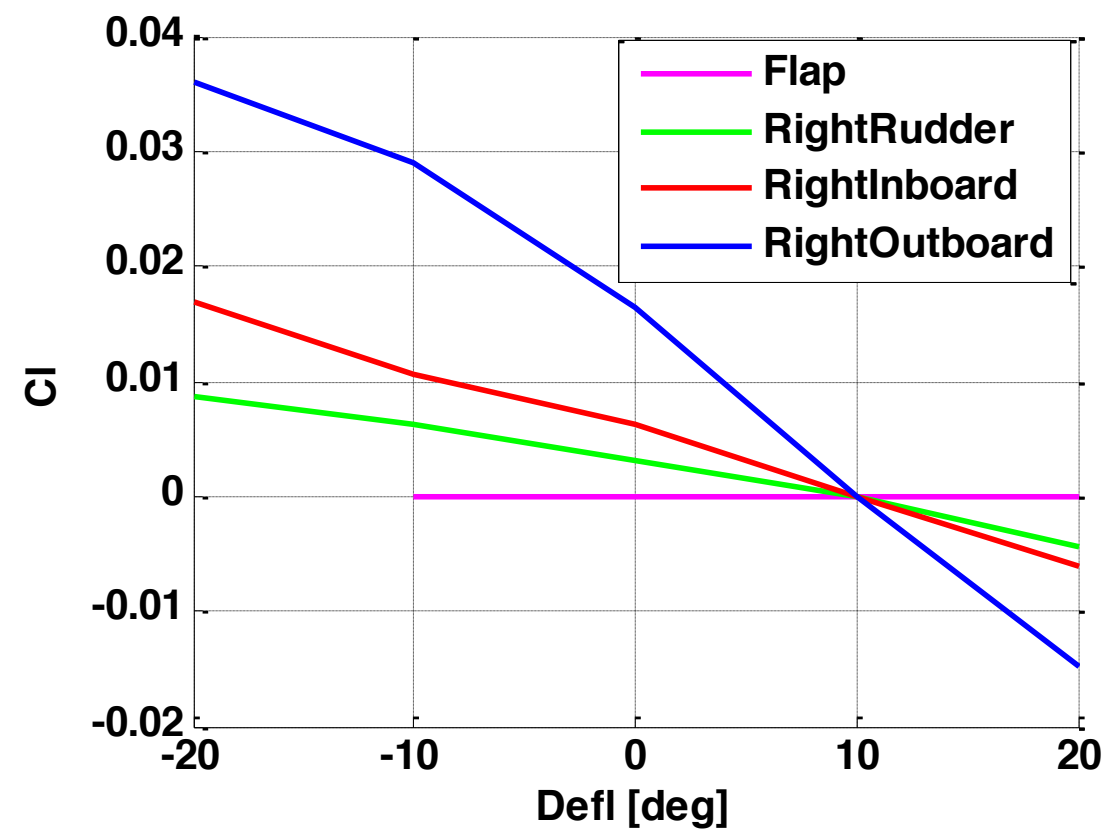

Figure 2-15: Roll effectiveness for control deflections.

Nice first order responses are seen in positive and negative pitch rate response to a $\mathrm{Q}$ doublet command in Figure 2-16. Maximum absolute steady state error is within 9\%, corresponding to acceptable tracking from the specified figures of merit. The frozen flight 
condition does not require any roll acceleration to cancel inherent plant dynamics. Therefore, enough control authority exists to provide pitch acceleration required to achieve a doublet command without saturating control surfaces.

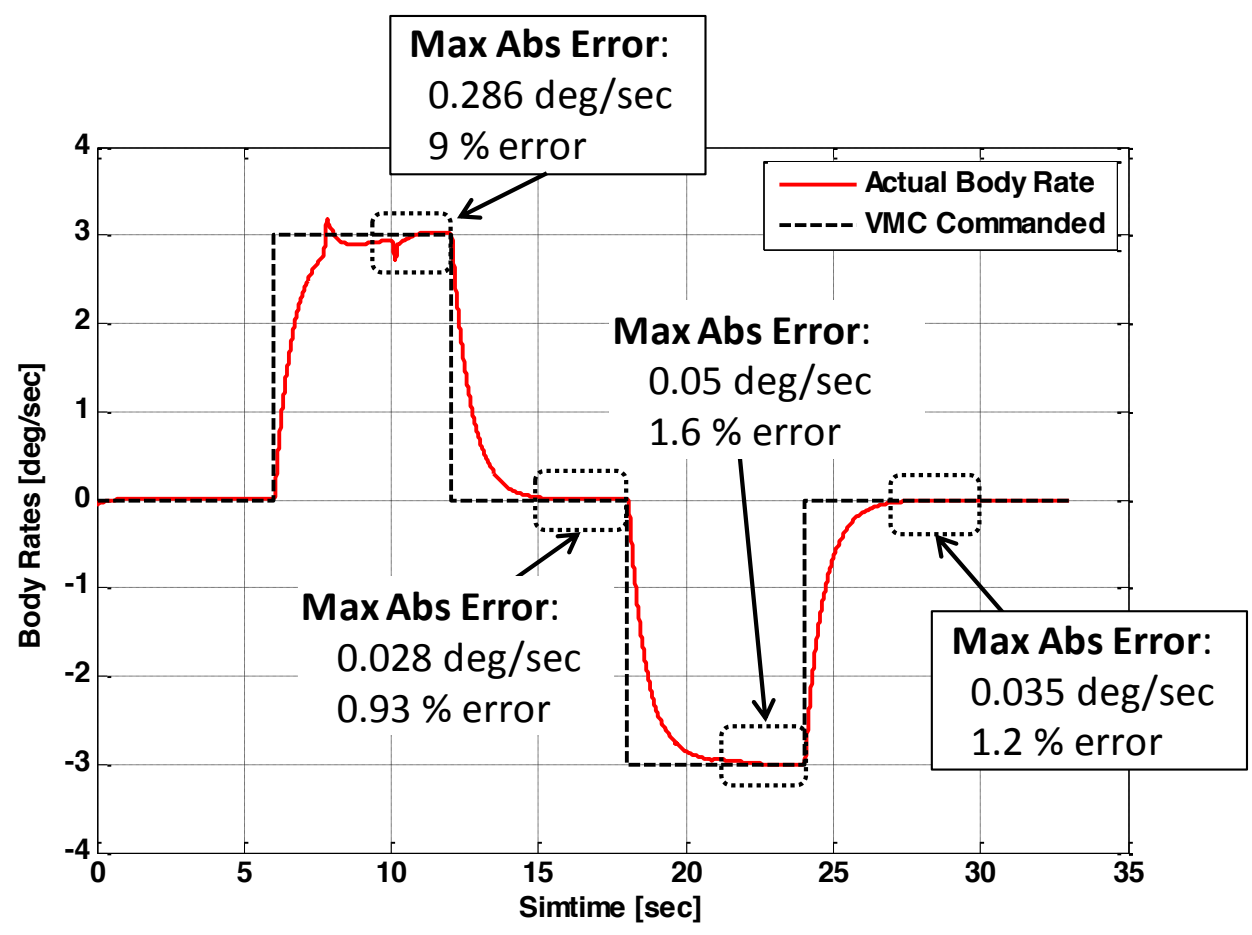

Figure 2-16: Pitch rate response to a doublet command.

The first positive step command to $3 \mathrm{deg} / \mathrm{sec}$ causes tiny spikes to occur in pitch rate response. This is due to the nonlinearity of control surface effectiveness in deflection and $\alpha$. Frozen flight condition means Mach number, and position in inertial and geodetic coordinates does not change, but $\alpha$ and $\beta$ is free to change with angular body rates. Commanding pitch rate causes the RLV to change $\alpha$, which consequently changes the local shape of a control effectiveness curve. Moreover, a control surface slope changes with control deflection. Figure 2-17 depicts how a control effectiveness slope changes drastically over a deflection break point. 


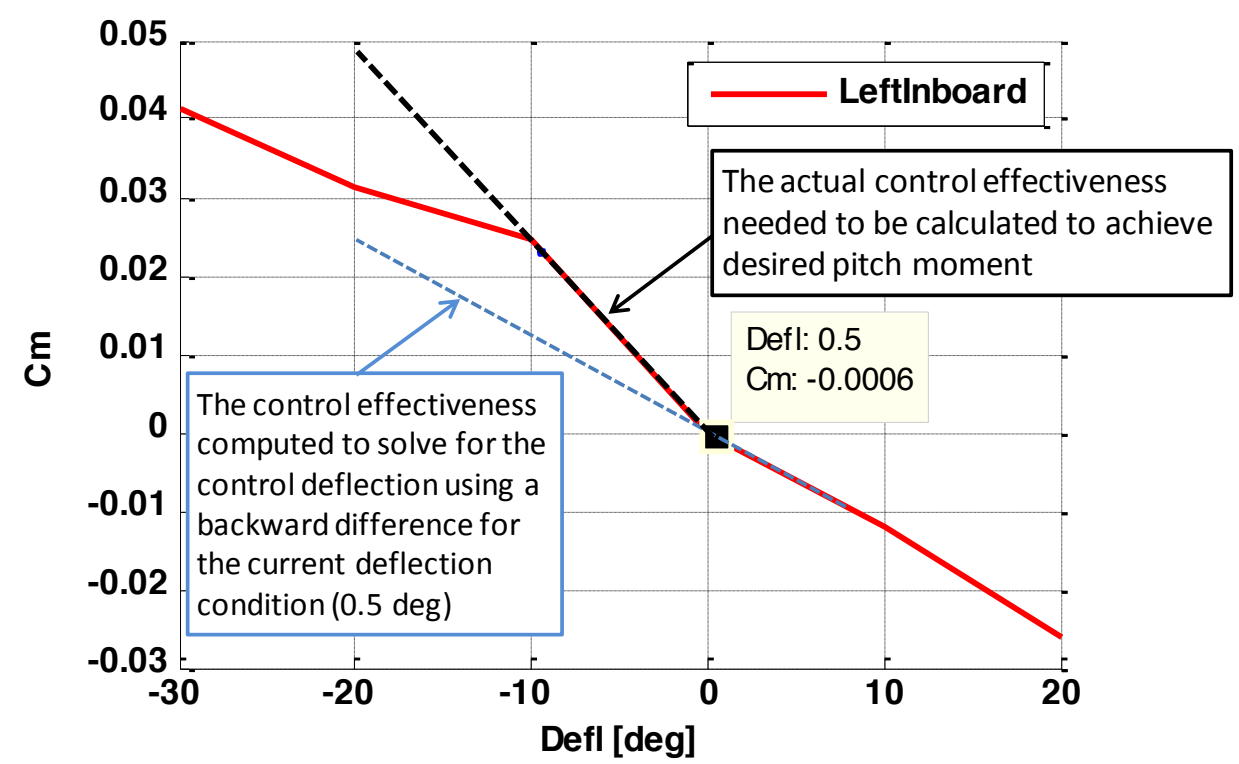

Figure 2-17: Control effectiveness computation can vary drastically at a break point.

Control effectiveness slope is computed using a backwards difference, so a slope is calculated from current flight conditions with 0 deg and 0.5 deg deflection if the current control deflection is $0.5 \mathrm{deg}$. If this local slope solves for a control deflection to achieve a desired positive moment, then the solution overestimates the required control deflection. The slope solved with a forward difference, shown as a black dotted line, should be used to more accurately solve for the control deflection to produce a positive moment. The blue dotted line, representing a backward difference control derivative, will solve for a deflection of -8 deg to achieve a desired moment coefficient of 0.01 , but this linear approximation will cause the RLV to produce a moment coefficient of 0.02 instead. Increase in pitch moment causes spikes in pitch rate response seen in the first step response.

Figure 2-18 shows a yaw rate response to a doublet command fed into the dynamic inversion controller. Control surfaces initially saturate at the first positive step input because control effectiveness slopes for each control surface at zero degrees $\beta$ are all zero. Control surface solution becomes very large, exceeding actuator limits, when a control effectiveness matrix, B, 
from Equation (2-32) is close to singular. Therefore, control surfaces saturate until the RLV experiences a little bit of $\beta$ so control surface derivatives for yaw increases enough to approach a valid solution to Equation (2-32). Initial axis saturation results in an integrator state to wind up when it is trying to close an error when control surfaces are saturated. Wind up causes overshoot in yaw rate. The prefilter integral term corrects for overshoot in a reasonable amount of time so absolute maximum error is $10 \%$ at 9 seconds into the simulation. Aside from the initial step command, the yaw rate steady state response is less than or equal to $0.12 \%$ maximum abolute steady state error, which is a good response according to the figures of merit.

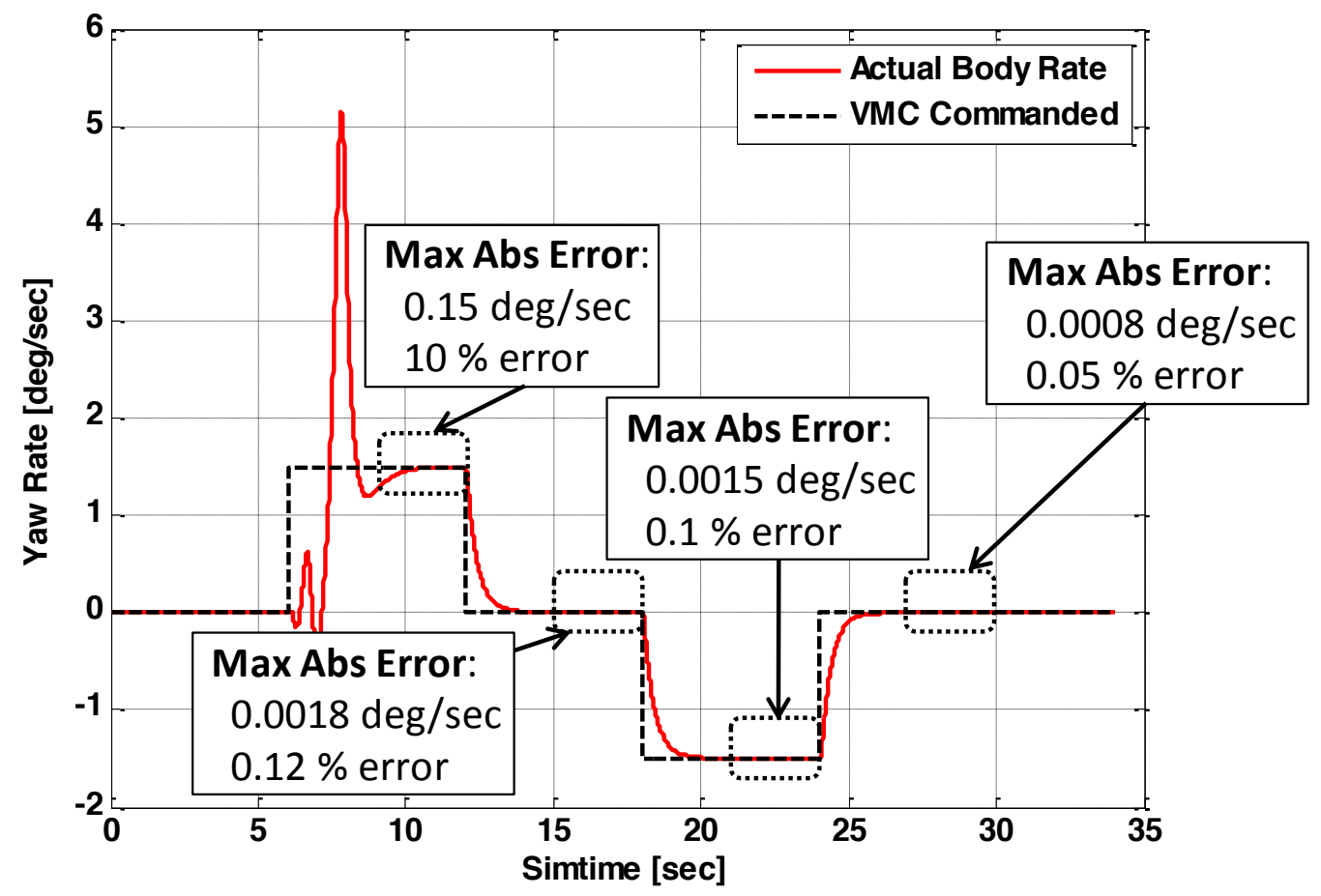

Figure 2-18: Yaw rate response to a doublet command.

Angular body rate doublet responses above will be used as the "baseline" to compare dynamic inversion controller performance with aerodynamic modelling error. 


\subsection{Backstepping Controller}

\subsubsection{Motivation}

Backstepping is another nonlinear control method so it eliminates the need to linearize a system about multiple operating points like gain scheduling ${ }^{19}$. A goal of the backstepping process is to bring a state vector to the origin by breaking down a nonlinear system into smaller subsystems. A virtual control law is designed for each subsystem by choosing a CLF to guarantee GAS of the subsystem. Each virtual control is integrated until an actual control variable is reached by "backstepping" each subsystem ${ }^{15}$. An advantage of backstepping over feedback linearization is that a stable controller can be designed with more robustness to parameter uncertainties because of the recursive nature of the design involving CLFs ${ }^{41}$. Transients from a desired state and a controller state are included directly into the CLF formulation in order to drive

the state to equilibrium ${ }^{20}$. Moreover, backstepping offers more flexibility in dealing with nonlinearities of a system. Feedback linearization requires canceling all nonlinearities even if the nonlinearity could provide stability to a system. This will sometimes lead to actuator saturations since additional control inputs required to cancel nonlinearities drive actuators past their limits. Backstepping allows a control designer to discriminate between nonlinear terms by cancelling "harmful" nonlinearities and taking advantage of nonlinearities that help with stability to stabilize the system ${ }^{23}$.

\subsubsection{Backstepping Formulation with Control Lyapunov Functions}

Backstepping is a recursive process that utilizes the LaSalle Yoshizawa theorem from Equation (1-84) with a CLF to guarantee GAS of a system from Equation (1-88) ${ }^{20}$. Backstepping 
can only be applied to nonlinear systems that are capable of being transformed into lower triangular form. Pure feedback form shown in Equation (2-38) is an example of a lower triangular form ${ }^{23}$.

$$
\begin{aligned}
& \dot{x_{1}}=f\left(x_{1}, x_{2}\right) \\
& \dot{x_{2}}=f\left(x_{1}, x_{2}, x_{3}\right) \\
& \vdots \\
& \dot{x_{\imath}}=f\left(x_{1}, x_{2}, x_{3}, \ldots, x_{i+1}\right) \\
& \vdots \\
& \dot{x_{n}}=f\left(x_{1}, x_{2}, x_{3}, \ldots, x_{i+1}, u\right)
\end{aligned}
$$

Systems that have states entering in an affine way are known as strict feedback form, where $u$ is the actual control ${ }^{23}$ :

$$
\begin{aligned}
& \dot{x_{1}}=f_{1}\left(x_{1}\right)+g_{1}\left(x_{1}\right) x_{2} \\
& \dot{x_{2}}=f_{2}\left(x_{1}, x_{2}\right)+g_{2}\left(x_{1}, x_{2}\right) x_{3} \\
& \vdots \\
& \dot{x_{l}}=f_{i}\left(x_{1}, x_{2}, x_{3}, \ldots, x_{i}\right)+g_{i}\left(x_{1}, x_{2}, x_{3}, \ldots, x_{i}\right) x_{4} \\
& \vdots \\
& \dot{x_{n}}=f_{n}\left(x_{1}, x_{2}, x_{3}, \ldots, x_{i}, x_{n}\right)+g_{n}\left(x_{1}, x_{2}, x_{3}, \ldots, x_{i}, x_{n}\right) u
\end{aligned}
$$

A backstepping control law is formed starting with a state farthest from the actual control, $u$. The first step is to consider $x_{2}$ as a virtual control of $x_{1}$ in a system below.

$$
\begin{aligned}
& \dot{x_{1}}=f\left(x_{1}\right)+g\left(x_{1}\right) x_{2} \\
& \dot{x_{2}}=u
\end{aligned}
$$

The objective is to find a virtual control law $\alpha_{l}\left(x_{1}\right)$ that stabilizes subsystem, Equation (2-40), by using the CLF:

$$
V_{1}\left(x_{1}\right)=\frac{1}{2} x_{1}^{2}
$$

If $\alpha_{l}\left(x_{1}\right)$ directly controls $x_{2}$, a time derivative of a CLF has to be negative definite in order to render the system in Equation (2-40) GAS. 


$$
\dot{V}_{1}=\frac{\partial V_{1}}{\partial x_{1}}\left(x_{1}\right)\left[f\left(x_{1}\right)+g\left(x_{1}\right) \alpha\left(x_{1}\right)\right]<0, \quad x_{1} \neq 0
$$

A residual term, $z$, is introduced, known as a virtual state. It is an error between $x_{2}$ and its desired value obtained from the control law ${ }^{20}$ expressed as.

$$
z=x_{2}-x_{2}^{\text {des }}=x_{2}-\alpha\left(x_{1}\right)
$$

A system is "backstepped" by considering an error state into a control input to derive the control law, $u$. An addition of an error state accounts for a transient error in command tracking. The idea is by forcing $z$ to zero or equilibrium, $x_{2}$ will tend toward a desired value making an entire system in Equation (2-40) and (2-41) stable ${ }^{17}$. The system from Equation (2-40) is rewritten in terms of an error state ${ }^{17}$.

$$
\dot{x}_{1}=f\left(x_{1}\right)+g\left(x_{1}\right)\left[\alpha_{1}\left(x_{1}\right)+z\right]
$$

The dynamics of an error state is expressed as ${ }^{17}$ :

$$
\dot{z}=u-\frac{\partial \alpha_{1}}{\partial x_{1}}\left(x_{1}\right)\left(f\left(x_{1}\right)+g\left(x_{1}\right)\left[\alpha\left(x_{1}\right)+z\right]\right)
$$

Then the CLF from (2-43) is augmented to include a term to penalize the error $\operatorname{state}^{17}, z$.

$$
V_{2}\left(x_{1}, z\right)=V_{1}\left(x_{1}\right)+\frac{1}{2} z^{2}
$$

Time derivative of the CLF above is taken in order to find a proper control law to make the CLF negative definite shown in Equation (2-48). 


$$
\begin{aligned}
\dot{V}_{2}= & \frac{\partial V_{1}}{\partial x_{1}}\left(x_{1}\right) \dot{x}_{1}+z \dot{z} \\
= & \frac{\partial V_{1}}{\partial x_{1}}\left(x_{1}\right)\left[f\left(x_{1}\right)+g\left(x_{1}\right)\left(\alpha\left(x_{1}\right)+z\right)\right]+z\left(u-\frac{\partial \alpha_{1}}{\partial x_{1}}\left(x_{1}\right)\left[f\left(x_{1}\right)+g\left(x_{1}\right)\left(\alpha\left(x_{1}\right)+z\right)\right]\right) \\
=\frac{\partial V_{1}}{\partial x_{1}}\left(x_{1}\right)[ & \left.f\left(x_{1}\right)+g\left(x_{1}\right) \alpha\left(x_{1}\right)\right] \\
& \quad+z\left(\frac{\partial V_{1}}{\partial x_{1}}\left(x_{1}\right) g\left(x_{1}\right)+u-\frac{\partial \alpha_{1}}{\partial x_{1}}\left(x_{1}\right)\left[f\left(x_{1}\right)+g\left(x_{1}\right)\left(\alpha\left(x_{1}\right)+z\right)\right]\right)
\end{aligned}
$$

The control law shown in Equation (2-49) will make the CLF negative definite as long as $\mathrm{c}>0$.

$$
u=-c z+\frac{\partial \alpha_{1}}{\partial x_{1}}\left(x_{1}\right)\left[f\left(x_{1}\right)+g\left(x_{1}\right)\left(\alpha\left(x_{1}\right)+z\right)\right]-\frac{\partial V_{1}}{\partial x_{1}}\left(x_{1}\right) g\left(x_{1}\right)
$$

For a chain of integrators, same steps will be repeated but with more virtual states to backstep to obtain the actual control. These three steps are summarized as follows ${ }^{17}$ :

1. Introduce virtual control $\alpha$ and an error state, $z$. Rewrite the current subsystem equation in terms of $\alpha$ and $z$.

2. Expand the CLF to reflect the presence of a virtual state

3. Choose an equation for the virtual control that makes CLF globally asymptotically stable.

These steps will be used in the following section to derive backstepping control laws used to control the RLV during approach and landing. 


\subsubsection{Backstepping Control Law Derivation}

The equations of motion are decoupled into a longitudinal axis and lateral-direction axes in order to build a backstepping controller. A longitudinal backstepping controller took altitude commands and creates a pitch rate command in body coordinates. A lateral-directional backstepping controller uses heading and $\beta$ commands to calculate body roll and yaw rate commands. The RLV banks to turn so $\beta$ command is kept constant at zero degrees to determine a required yaw command. Control surface actuator dynamics are ignored when designing the controller, assuming they are fast enough to disregard.

\subsubsection{Longitudinal Axis Backstepping Formulation}

Three feedback loops are designed to follow a trajectory. Altitude error is closed using $\gamma$, then $\alpha$ is used to close the loop on $\gamma$, and finally the pitch rate is used to close the error on $\alpha$ as seen in Figure 2-19.

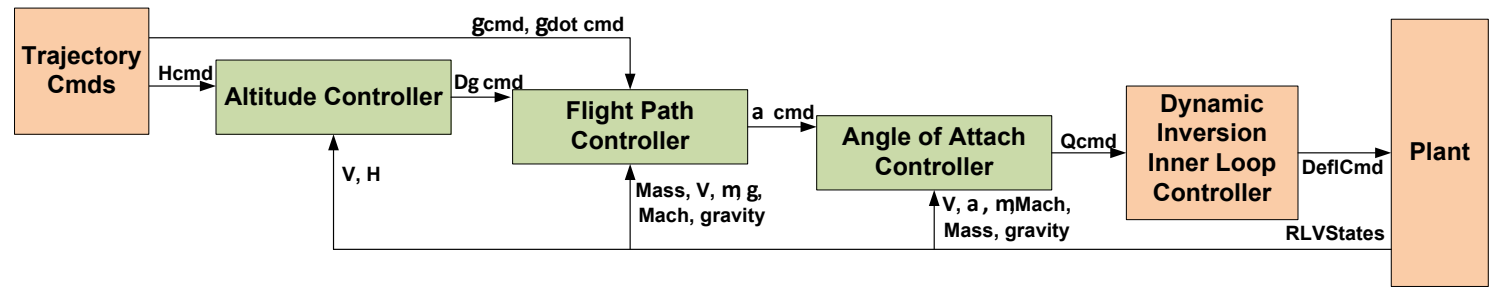

Figure 2-19: Functional architecture for the longitudinal backstepping controller.

The dynamic equations used to derive backstepping control laws are as follows ${ }^{15}$ :

$$
\begin{gathered}
\dot{H}=V \sin \gamma \\
\dot{\gamma}=\frac{L \cos \mu}{m V}-\frac{g}{V} \cos \gamma \\
\dot{\alpha}=-\dot{\gamma}+Q=\frac{g}{V} \cos \gamma-\frac{L \cos \mu}{m V}+Q
\end{gathered}
$$


These simple 3 DoF equations assume $\beta$ is equal to zero degrees. In order to use a backstepping formulation, equations are put into strict feedback form, where $x_{1}$ represents altitude, $x_{2}$ is $\gamma, x_{3}$ is $\alpha, u$ is the control variable, body pitch rate, Q. The lift in Equation (2-51) and (2-52) is assumed to be a linear function of $\alpha$ to transform the equation in strict feedback form below.

$$
\begin{gathered}
\dot{x}_{1}=V \sin \left(x_{2}\right) \\
\dot{x}_{2}=-\frac{g}{V} \cos \left(x_{2}\right)+\frac{L_{\alpha}\left(x_{3}\right) \cos \mu}{m V} \\
\dot{x_{3}}=\frac{g}{V} \cos \left(x_{2}\right)-\frac{L_{\alpha}\left(x_{3}\right) \cos \mu}{m V}+u \\
u=Q
\end{gathered}
$$

Where $\dot{H}$ is equal to $\dot{x}_{1}, \dot{\gamma}$ is equal to $\dot{x}_{2}$, and $\dot{\alpha}$ is equal to $\dot{x}_{3}$, and $L_{\alpha}$ is the linear lift curve slope.

First the flight path angle, $x_{2}$, is used as a virtual control of altitude, $x_{1}$, using the control law, $\alpha_{1}$.

$$
x_{2 d e s}=\alpha_{1}\left(x_{1}\right)
$$

A CLF is chosen to stabilize subsystem, $x_{1}$ :

$$
V_{1}=\frac{1}{2} x_{1}^{2}
$$

The time derivative of the CLF has to be negative definite in order to make the system GAS.

$$
\dot{V}_{1}=x_{1} \dot{x}_{1}
$$

Substituting $\dot{x}_{1}$ dynamics from Equation (2-53) into the Equation (2-59) above, 


$$
\dot{V}_{1}=x_{1}\left(V \sin \alpha_{1}\right)<0
$$

The virtual controller was designed as Equation (2-61) to make the CLF in Equation (2-60) negative definite.

$$
\alpha_{1}=\sin ^{-1}\left(\frac{-k_{1} x_{1}}{V}\right)
$$

Substituting $\alpha_{1}$ into the CLF from Equation (2-60) gives Equation (2-62), proving the CLF is negative definite.

$$
\begin{aligned}
\dot{V}_{1}=- & k_{1} x_{1}{ }^{2} \\
& \text { where } k_{1}>0
\end{aligned}
$$

The residual between the desired $x_{2}$ state obtained from the virtual control, from Equation (2-57), and the actual $x_{2}$ state is introduced by $z_{1}$

$$
\begin{aligned}
& z_{1}=x_{2}-x_{2 d e s} \\
& z_{1}=x_{2}-\alpha_{1}
\end{aligned}
$$

Another virtual control, $\alpha_{2}$, is created using the angle of attack, $x_{2}$, as the virtual state to control flight path angle, $x_{3}$.

$$
x_{3 d e s}=\alpha_{2}\left(x_{2}\right)
$$

After establishing the residual virtual state, $z_{1}$, and the new virtual control, $\alpha_{2}$, the system is "backstepped" and the dynamic equation for $x_{2}$ is rewritten in terms of $z_{1}$ and $\alpha_{2}$ shown in Equation (2-66)

$$
\dot{x}_{2}=-\frac{g}{V} \cos \left(z_{1}+\alpha_{1}\right)+\frac{L_{\alpha}\left(\alpha_{2}\right) \cos \mu}{m V}
$$


The dynamics of the virtual state is derived by taking the time derivative of $z_{1}$. The states, $x_{2}$ and $x_{3}$ are rewritten in terms of the virtual state $z_{1}$, and virtual controls $\alpha_{1}$ and $\alpha_{2}$.

$$
\begin{gathered}
\dot{z}_{1}=\dot{x}_{2}-\dot{\alpha}_{1} \\
\dot{z}_{1}=-\frac{g}{V} \cos \left(z_{1}+\alpha_{1}\right)+\frac{L_{\alpha}\left(\alpha_{2}\right) \cos \mu}{m V}-\frac{\partial \alpha_{1}}{\partial x_{1}} \dot{x}_{1}
\end{gathered}
$$

The CLF from Equation (2-59) is augmented with the residual state $z_{l}$ to stabilize the system from Equations (2-53) and (2-54):

$$
\begin{aligned}
& V_{2}=V_{1}+\frac{1}{2} z_{1}^{2} \\
& \dot{V}_{2}=\dot{V}_{1}+z_{1} \dot{z}_{1}
\end{aligned}
$$

Substituting $\dot{V}_{1}$ with Equation (2-62) and $\dot{z}_{1}$ with Equation (2-68):

$$
\dot{V}_{2}=-k_{1} x_{1}^{2}+z_{1}\left(-\frac{g}{V} \cos \left(z_{1}+\alpha_{1}\right)+\frac{L_{\alpha}\left(\alpha_{2}\right) \cos \mu}{m V}-\frac{\partial \alpha_{1}}{\partial x_{1}} \dot{x}_{1}\right)
$$

The virtual control, $\alpha_{2}$, has to be designed so that $\dot{V}_{2}$ is negative definite.

$$
\alpha_{2}=L_{\alpha}{ }^{-1}\left(\frac{m V\left(-k_{2} z_{1}+\frac{g}{V} \cos \left(z_{1}+\alpha_{1}\right)\right)}{\cos \mu}\right)
$$

Where $\mathrm{L}^{-1}$ indicates taking the inverse of the lift equation so that the values inside the parenthesis equals the lift. The virtual control, $\alpha_{2}$, can be selected to be linear in terms of $z_{1}$, as in Equation (2-65), as long as $\dot{\alpha_{1}}$ is bounded such that:

$$
k_{2}>\max _{x_{1}} \frac{\partial \alpha_{1}}{\partial x_{1}} \dot{x}_{1}
$$


This condition requires that the inner loop feedback gain, $k_{2}$, must be greater than the outer loop feedback gain, $k_{l}^{20}$. Substituting Equation (2-72) into Equation (2-71) gives a negative definite CLF, shown in Equation (2-74), as long as condition from Equation (2-73) is satisfied.

$$
\dot{V}_{2}=-k_{1} x_{1}^{2}-k_{2} z_{1}^{2}-\frac{\partial \alpha_{1}}{\partial x_{1}} \dot{x}_{1} z_{1}
$$

The new virtual state, $z_{2}$, is defined as the error between desired $x_{3}$ value from the virtual control and the actual $x_{3}$ state.

$$
\begin{aligned}
& z_{2}=x_{3}-x_{3 \text { des }} \\
& z_{2}=x_{3}-\alpha_{2}
\end{aligned}
$$

The system from Equation (2-53) through Equation (2-56) is backstepped again by rewriting the dynamics of the angle of attack, $x_{3}$ from Equation (2-55), in terms of the virtual states, $z_{1}$ and $z_{2}$, and virtual control $\alpha_{1}$ and $\alpha_{2}$, as in Equation (2-77). The dynamics of the virtual state $z_{2}$ is derived in Equation (2-79) by taking the time derivative of Equation (2-75).

$$
\begin{gathered}
\dot{x}_{3}=\frac{g}{V} \cos \left(z_{1}+\alpha_{1}\right)-\frac{L_{\alpha}\left(z_{2}+\alpha_{2}\right) \cos \mu}{m V}+u \\
\dot{z}_{2}=\dot{x}_{3}-\dot{\alpha}_{2}
\end{gathered}
$$

Substituting $\dot{x}_{3}$ with Equation (2-77):

$$
\dot{z}_{2}=\frac{g}{V} \cos \left(z_{1}+\alpha_{1}\right)-\frac{L_{\alpha}\left(z_{2}+\alpha_{2}\right) \cos \mu}{m V}+u-\frac{\partial \alpha_{2}}{\partial z_{1}} \dot{z}_{1}
$$

A CLF is chosen to augment the previous CLF from Equation (2-69) to reflect the presence of the new virtual state $z_{2}$. 


$$
V_{3}=V_{2}+\frac{1}{2} z_{2}^{2}
$$

The time derivative of the CLF is derived by using the chain rule.

$$
\dot{V}_{3}=\dot{V}_{2}+z_{2} \dot{z}_{2}
$$

Substituting Equation (2-74) and Equation (2-79) into the equation above:

$$
\begin{aligned}
\dot{V}_{3}=-k_{1} x_{1}{ }^{2}-k_{2} z_{1}{ }^{2}- & \frac{\partial \alpha_{1}}{\partial x_{1}} \dot{x}_{1} z_{1} \\
& +z_{2}\left(\frac{g}{V} \cos \left(z_{1}+\alpha_{1}\right)-\frac{L_{\alpha}\left(z_{2}+\alpha_{2}\right) \cos \mu}{m V}+u-\frac{\partial \alpha_{2}}{\partial z_{1}} \dot{z}_{1}\right)
\end{aligned}
$$

The actual control of the system, $u$, can be constructed as Equation (2-83) so that the CLF in Equation (2-82) is negative definite, which guarantees that the system in Equation (2-53) through Equation (2-56) is GAS.

$$
u=-k_{3} z_{3}-\frac{g}{V} \cos \left(z_{1}+\alpha_{1}\right)+\frac{L_{\alpha}\left(z_{2}+\alpha_{2}\right) \cos \mu}{m V}
$$

The control, $u$, would make the CLF negative definite as long as $u$ dominates the $\dot{\alpha}_{2}$ term. This condition is guaranteed when $\dot{\alpha}_{2}$ is bounded by ${ }^{15}$ :

$$
k_{3}>\max _{z_{2}} \frac{\partial \alpha_{2}}{\partial z_{2}} \dot{z}_{2}
$$

Which can be satisfied if ${ }^{15}$ :

$$
k_{3}>k_{2}>k_{1}
$$

To summarize, the backstepping control law for the longitudinal axis is derived with three feedback loops, using the virtual controls, $\alpha_{1}, \alpha_{2}$, and the control variable, $u$, to close the 
error on altitude, $\gamma$, and $\alpha$. The altitude loop control law in Equation (2-86) ${ }^{15}$ uses $\gamma$ as the virtual control of altitude. It takes the altitude error and develops a corrective $\gamma$ command, derived from Equation (2-61).

$$
\begin{aligned}
& \Delta \gamma_{c m d}=\sin ^{-1}\left(\frac{\Delta \dot{H}_{d e s}}{V}\right) \\
& \text { where } \Delta \dot{H}_{\text {des }}=k_{1}\left(H_{c m d}-H_{\text {actual }}\right)
\end{aligned}
$$

The $\gamma$ control law, Equation $(2-87)^{15}$, uses a as the virtual control to close the error on $\gamma$. The $\alpha$ command is computed from the $\gamma$ error using the control law developed from backstepping in Equation (2-72).

$$
\begin{gathered}
\alpha_{c m d}=L_{\alpha}{ }^{-1}\left(\frac{m V\left(\dot{\gamma}_{d e s}-\frac{g}{V} \cos \gamma_{\text {actual }}\right)}{\cos \mu}\right) \\
\text { where } \dot{\gamma}_{\text {des }}=k_{2}\left(\gamma_{c m d}+\Delta \gamma_{c m d}-\gamma_{\text {actual }}\right)
\end{gathered}
$$

The lift curve equation can be inverted to find which $\alpha$ would give the lift value inside the parenthesis in Equation (2-87) since the lift is assumed to be a function of $\alpha$. The CLF derivation assumes that the lift curve can be perfectly inverted to find the proper $\alpha$ to produce the required $\operatorname{lift}^{15}$. In reality, there may be error associated with finding the right $\alpha$ command because the aerodynamic curves are nonlinear. The implementation of the inversion is explained in the next Section, 2.2.4. After backstepping through the system of equations, Equations (2-53) through Equation (2-56), the final feedback loop arrives at the actual control, pitch rate, Q. The $\alpha$ control law, shown in Equation (2-88) ${ }^{15}$, calculates the Q derived by Equation (2-83) to make the CLF GAS.

$$
\begin{aligned}
& Q_{c m d}=\dot{\alpha}_{d e s}-\frac{g}{V} \cos \gamma_{a c t u a l}+\frac{L_{\text {actual }} \cos \mu}{m V} \\
& \text { where } \dot{\alpha}_{d e s}=k_{3}\left(\alpha_{c m d}-\alpha_{\text {actual }}\right)
\end{aligned}
$$


The proportional gains for each feedback loop has to satisfy the condition given in Equation (2-85) in order to guarantee GAS for the system of equations. The CLFs for the pitch-axis controller only includes states in the longitudinal plane for simplicity. Therefore, only part of the whole system is proven to be GAS. Dynamic inversion inner loop controller states, the rotational dynamic states, and states for the lateral-directional plane of motion were not included in the CLF formulation. The robustness analysis in Section 3 will quantify the stability properties of the system if parts of the whole system are individually proven to be GAS. 


\subsubsection{Longitudinal Axis Backstepping Implementation into Simulink}

Figure 2-20 depicts how the three feedback loops described in the previous section are implemented into Simulink.

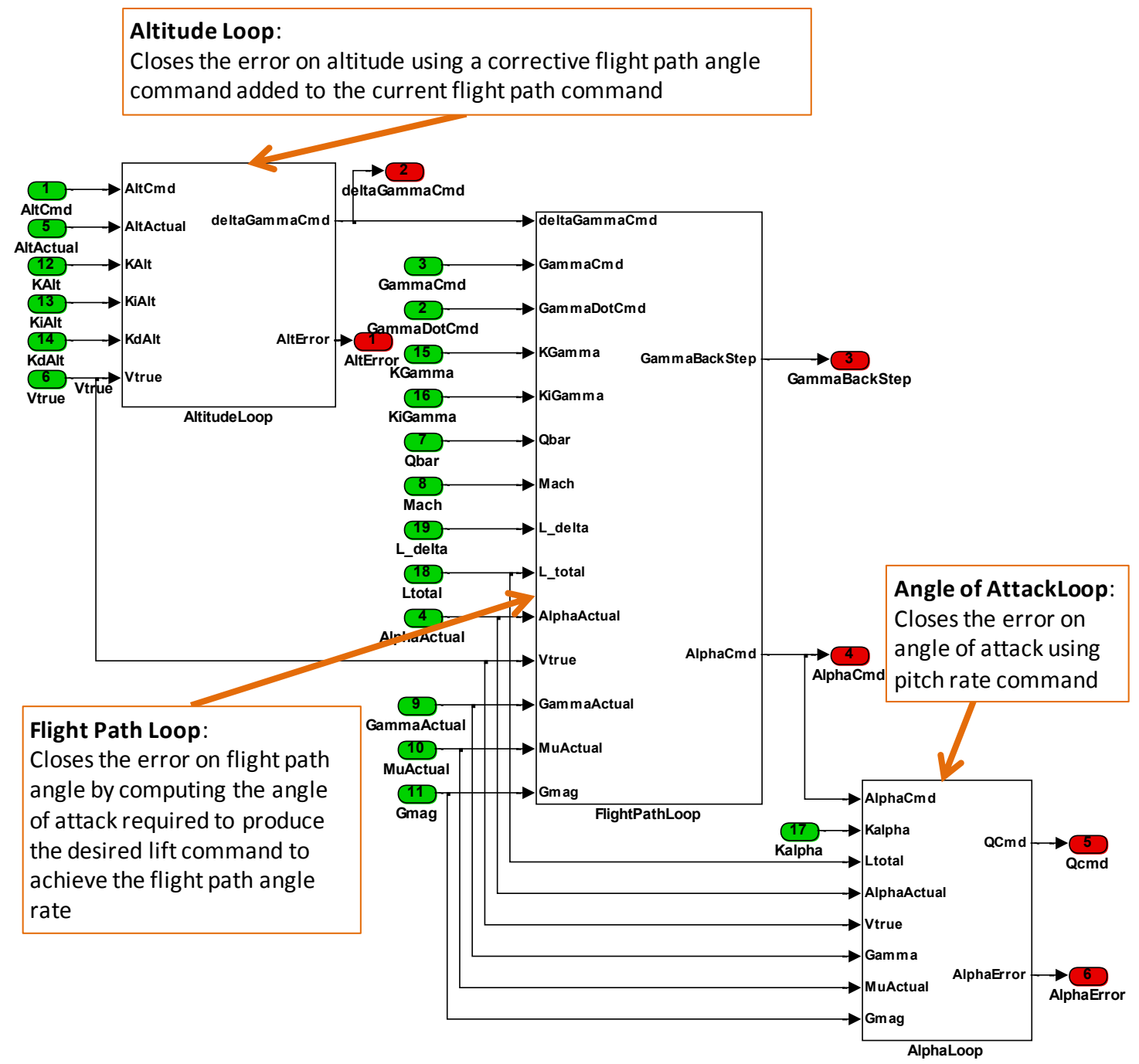

Figure 2-20: Simulink implementation of the pitch-axis backstepping controller.

The altitude control law from Equation (2-86) is implemented as Figure 2-21. Integrator and derivative states are added to the original feedback control law in order to compensate for aerodynamic modeling error in the controller. These gains are defined later in Section 2.2.4.1. A saturation limit is placed on the computed corrective $\gamma$ command in case the altitude error gets so 
large that the vehicle commands a corrective $\gamma$ exceeding vehicle capabilities for an unpowered approach and landing.

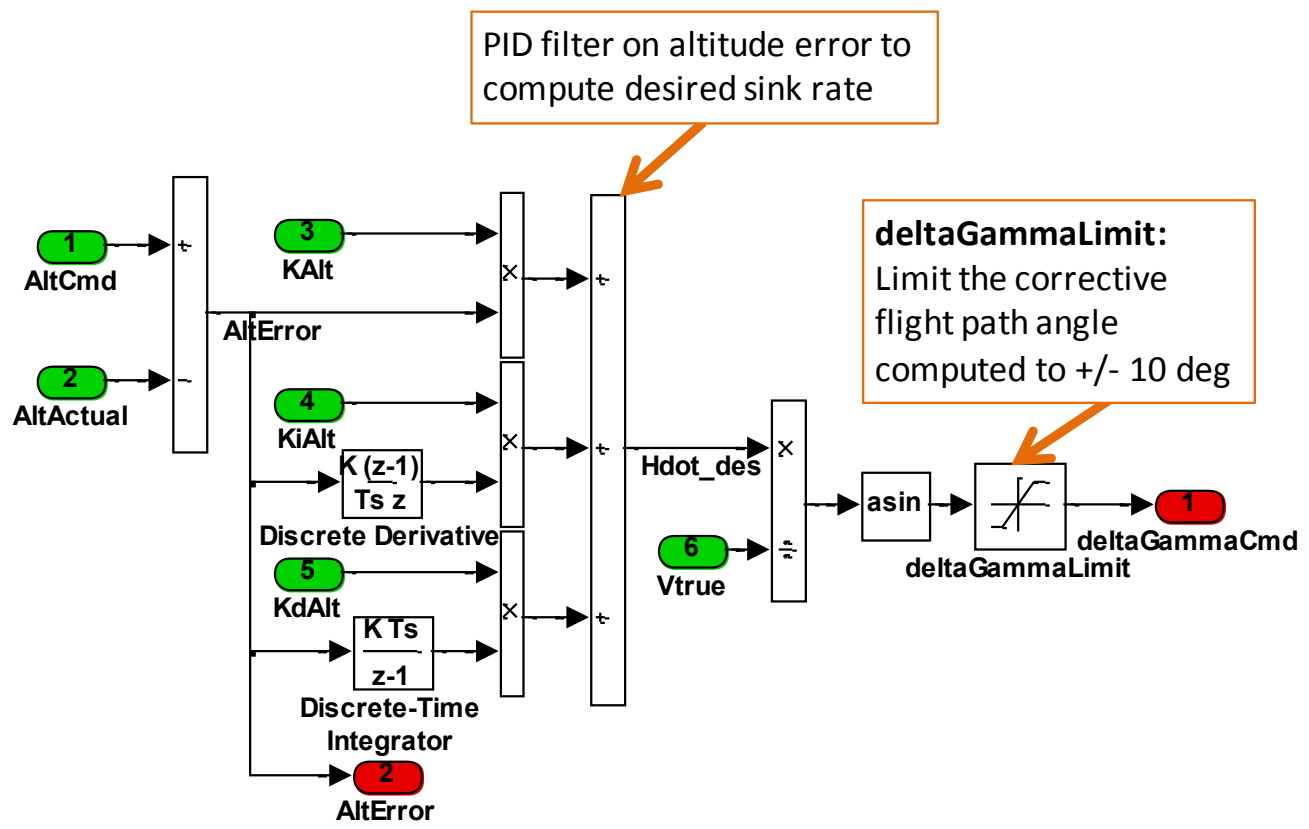

Figure 2-21: The altitude loop implementation into Simulink.

The $\gamma$ control law is illustrated in Figure 2-22. An integral state is added to the proportional state from Equation (2-87) in order to close the error caused by aerodynamic modeling mismatch between the controller and the plant.

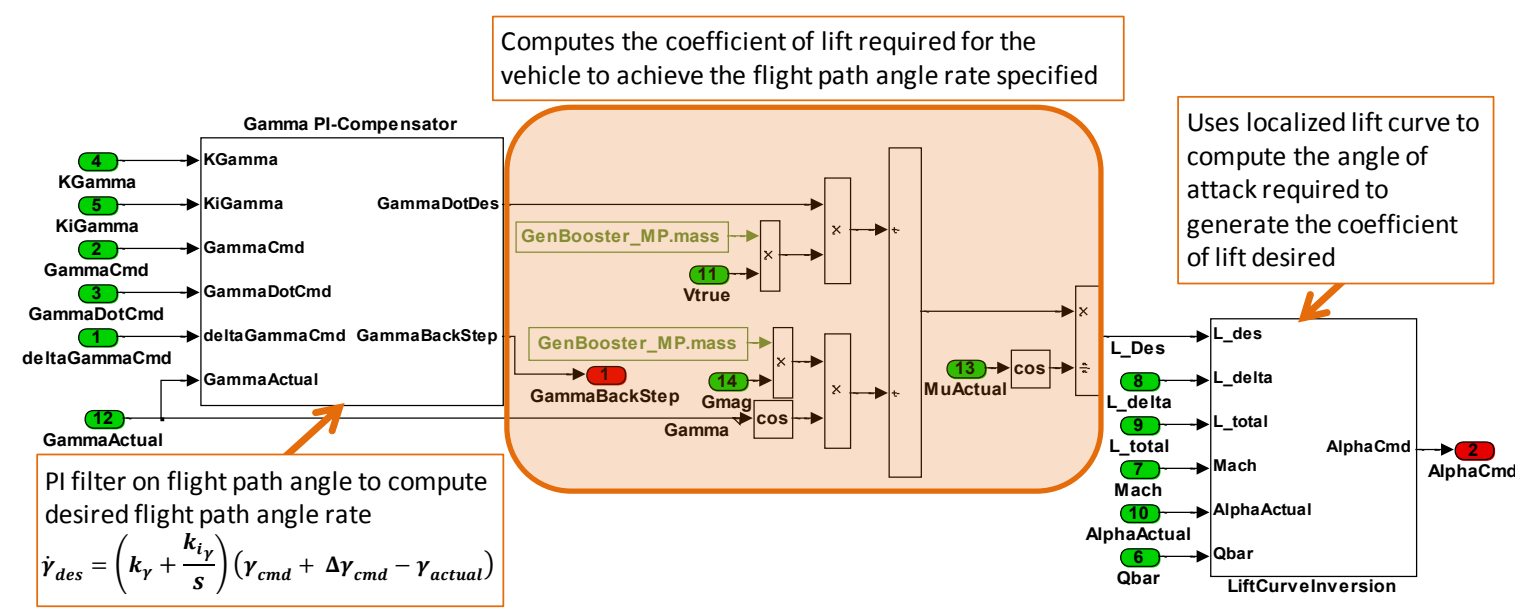

Figure 2-22: The flight path controller implementation into Simulink. 
The lift for the bare airframe and the control surfaces for the RLV are nonlinear functions of $\alpha$, Mach, $\beta$, and $\delta$. However, the control law formulation from Equation (2-87) assumes that the lift curve is a linear function of $\alpha$. The same concept used to linearize the control surface derivatives, in Section 2.1.4, has to be applied to the lift curve slope in order to use a linear control law. The static lift curve is linearized about a local flight condition using an epsilon term to compensate for the errors contributed to linearizing the curve.

The total vehicle lift at a current flight condition, $L_{\text {total }}$, can be separated between the lift contribution from the control surfaces, $L_{\delta}$, and the lift contribution from the bare airframe, $L_{B A E}$ :

$$
L_{\text {total }}=L_{\delta}(P, \alpha)+L_{B A E}(P, \alpha)
$$

Where $\mathrm{P}$ are flight condition parameters like Mach, $\beta$, and $\delta$ in degrees. The dynamic equations used for formulating the CLF assume that $\beta$ is constant at zero degrees since the vehicle is suppose to be flying at $\beta$ equal to zero to minimize drag effects. Therefore, the vehicle lift becomes a function of $\alpha$ and Mach only, with $\beta$ constant at zero degrees. The dynamic inversion controller solves for the proper control deflection needed to track the inner loop commands so $L_{\delta}$ term is treated as a constant with respect to the current $\alpha$. Then, Equation (2-89) can be rewritten in terms of $\alpha$ dependent lift and the lift contribution from the control surfaces shown as Equation $(2-90)$.

$$
L_{\text {total }}=L_{\delta}+L_{\alpha_{B A E}} \Delta \alpha+\varepsilon_{\alpha}(P, \alpha)
$$

The $L_{\alpha}$ is the localized static lift derivative with respect to $\alpha$ at a current flight condition, and $\varepsilon_{\alpha}$ is the penalty term added because of the error incurred from linearizing the lift curve at the 
current $\alpha$ and Mach. The $\Delta \alpha$ term is the change in $\alpha$ from the current $\alpha$. Then, the penalty term can be computed by rearranging Equation (2-90):

$$
\varepsilon_{\alpha}(P, \alpha)=L_{\text {total }}-L_{\delta}-L_{\alpha B E} \Delta \alpha
$$

The linear lift curve slope approximation from Equation (2-90) is used to reformulate the control law from Equation (2-87) to solve for the $\alpha$ command.

$$
\begin{gathered}
L_{\text {des }}=\left(\frac{m V\left(\dot{\gamma}_{\text {des }}-\frac{g}{V} \cos \gamma_{\text {actual }}\right)}{\cos \mu}\right) \\
L_{\delta}+L_{\alpha_{B A E} \Delta \alpha_{c m d}+\varepsilon_{\alpha}(P, \alpha)}=\left(\frac{m V\left(\dot{\gamma}_{\text {des }}-\frac{g}{V} \cos \gamma_{\text {actual }}\right)}{\cos \mu}\right) \\
L_{\alpha_{B A E} \Delta \alpha_{c m d}}=\left(\frac{m V\left(\dot{\gamma}_{\text {des }}-\frac{g}{V} \cos \gamma_{\text {actual }}\right)}{\cos \mu}\right)-\varepsilon_{\alpha}(P, \alpha)-L_{\delta} \\
\Delta \alpha_{c m d}=L_{\alpha_{B A E}}\left[\left(\frac{m V\left(\dot{\gamma}_{\text {des }}-\frac{g}{V} \cos \gamma_{\text {actual }}\right)}{\cos \mu}\right)-\varepsilon_{\alpha}(P, \alpha)-L_{\delta}\right]
\end{gathered}
$$

The total $\alpha$ command to feeding into the $\alpha$ loop is computed by:

$$
\alpha_{c m d}=\Delta \alpha_{c m d}+\alpha_{c u r r e n t}
$$


The implementation of the reformulated control law from Equation (2-92) is depicted in Figure $2-23$

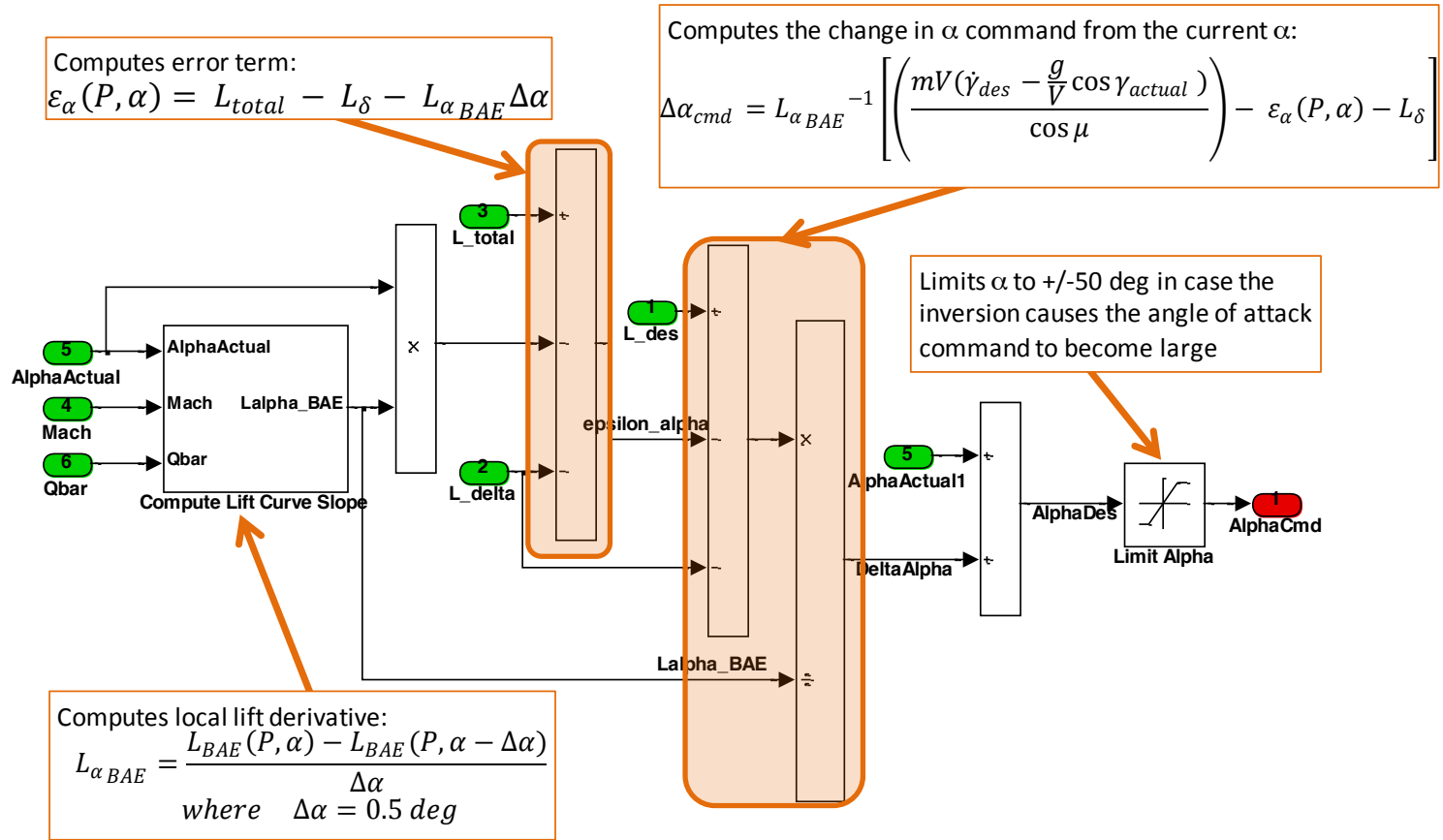

Figure 2-23: Lift curve inversion implementation into Simulink.

The local lift curve slope is computed similarly to the control surface effectiveness in the dynamic inversion formulation. A backward difference is taken between the static lift at the current Mach and $\alpha$ and the static lift at the current Mach and 0.5 degrees less than the current $\alpha$. The $\alpha$ command computed from Equation (2-96) is limited between $+/-50$ degrees in case $\gamma$ error drives the desired lift command to a large value causing the vehicle to command $\alpha$ past trimmable regions.

Figure 2-24 depicts vehicle states used in the altitude and $\gamma$ loops. A negative altitude error adds a negative corrective $\gamma$ to an existing trajectory $\gamma$ command in red, resulting in the $\gamma$ commanded by the backstepping controller, represented by $\gamma_{\text {Backstep. This }} \gamma_{\text {Backstep is used as the }}$ command to generate a proper $\alpha$ command to close the error on the vehicle's $\gamma$ error. 


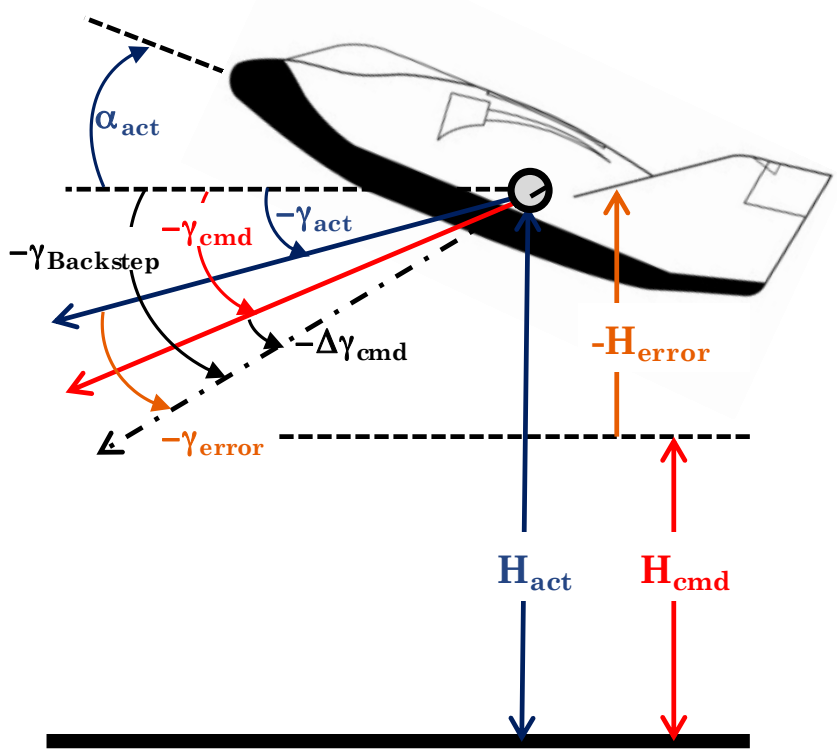

Figure 2-24: Vehicle state definition used in the pitch axis backstepping controller.

Figure 2-25 depicts how the $\alpha$ command from Figure 2-23 feeds into the $\alpha$ loop formulated as Equation (2-88) to compute the pitch rate, Q, command.

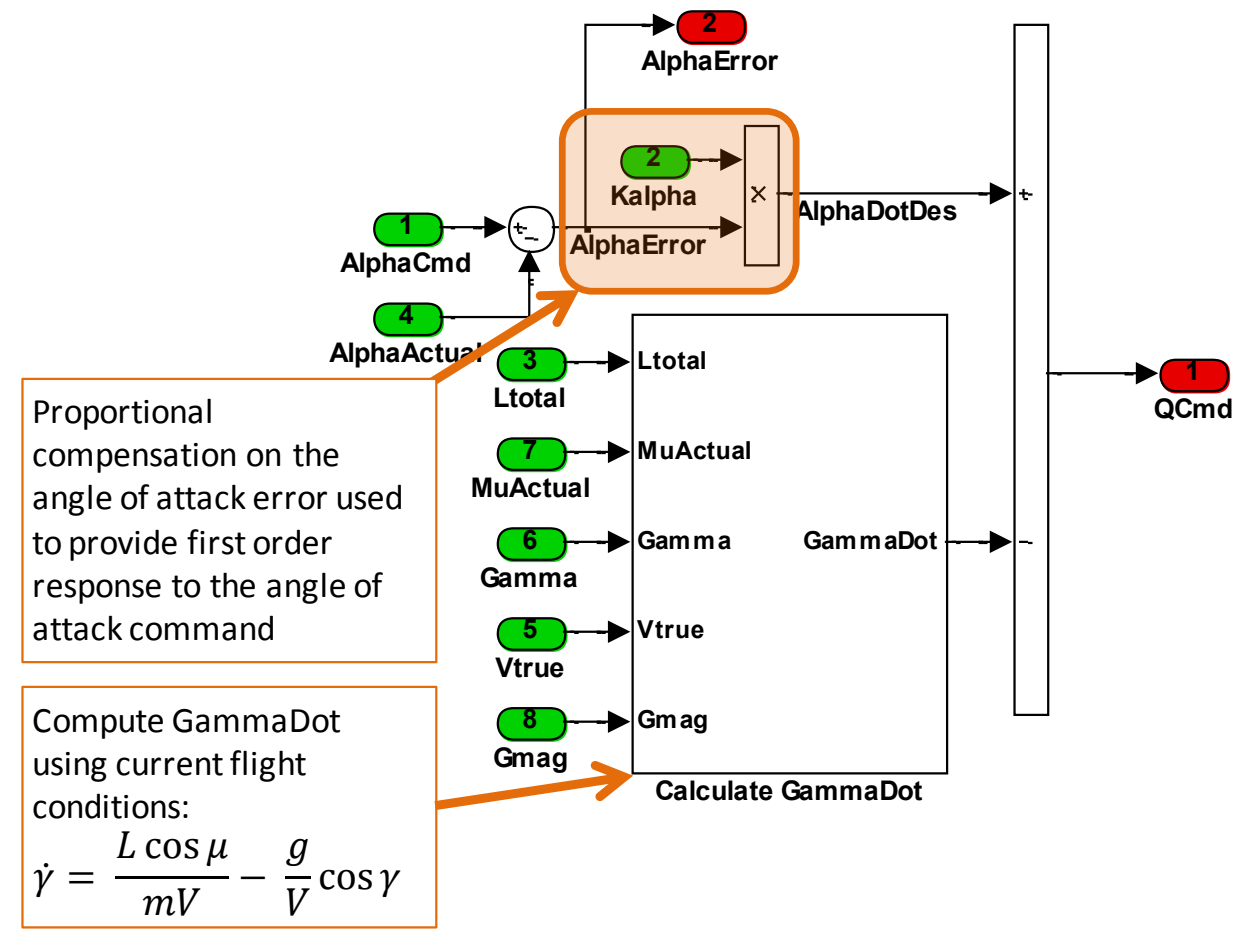

Figure 2-25: Angle of attack control law implementation into Simulink. 


\subsubsection{Lateral-Directional Axis Backstepping Formulation}

The lateral-directional axis backstepping formulation is further separated between the roll axis and yaw axis. The body roll rate, $\mathrm{P}$, is used as the control to close the error on heading, $\chi$, and crosstrack error. The body yaw rate, $R$, is used as the control to keep $\beta$ equal to zero. Figure 2-26 depicts the block diagrams of the control laws used to control the lateral axis of the vehicle. The details of the control laws for each block is discussed below.

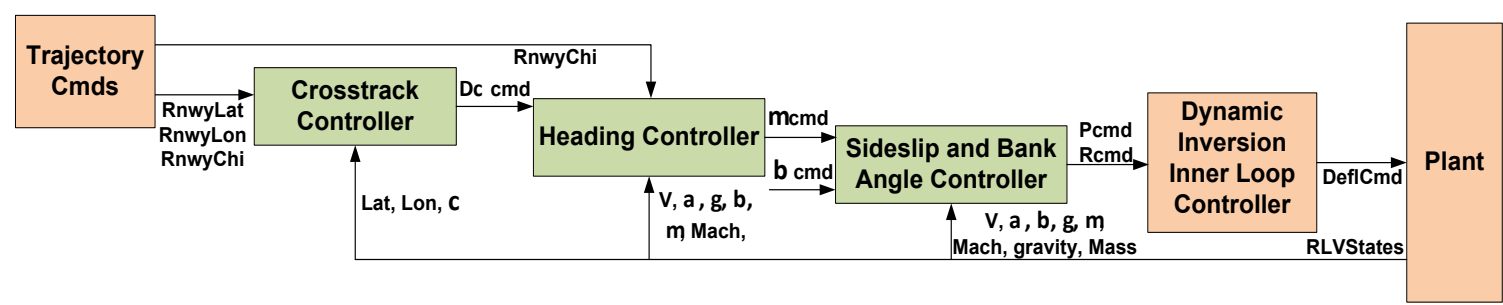

Figure 2-26: Functional architecture for the lateral-directional axis backstepping controller.

The lateral-directional axis control starts with defining the crosstrack error in order to determine the corrective heading command derived from a PD control on the error. Following the proper runway $\chi$ only will not be enough to get to the latitude and longitude point of the runway. The vehicle will accumulate a crosstrack error if the vehicle deviates from the runway $\chi$ for a short period. The crosstrack error will persist even if the vehicle corrects its $\chi$ error unless there is a feedback loop on crosstrack.

The crosstrack error is computed using the range and azimuth between the current vehicle position and the runway position and azimuth on the Earth's surface. The range and azimuth between two positions are calculated using the inverse vincenty formulae. The inverse vincenty formulae uses an iterative method to compute the geographical range and azimuth to an accuracy within 0.02 inches on the WGS-84 ellipsoid. Appendix B contains the Matlab code developed to 
implement this calculation in an Embedded Matlab block. Figure 2-27 depicts how the parameters computed from the inverse vincenty formulae are used to define the crosstrack error.

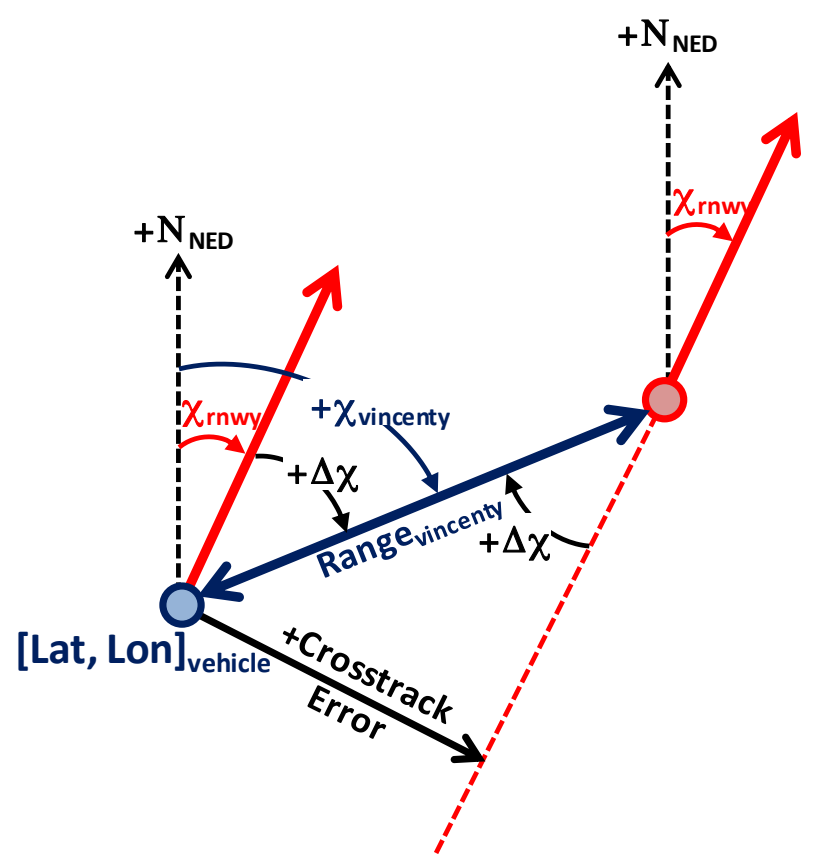

Figure 2-27: Crosstrack error definition using parameters computed from inverse vincenty.

$\chi$ computed from the inverse vincenty equations is defined from the north axis in NED coordinates. The range is a direct distance over an ellipsoid surface to the runway latitude and longitude position. The geometry in Figure 2-27 shows crosstrack error is computed by:

$$
\begin{aligned}
& \text { XtrackErr }=R_{\text {vincenty }} \Delta \chi \\
& \text { where } \Delta \chi=\chi_{\text {vincenty }}-\chi_{r n w y}
\end{aligned}
$$

Where XtrackErr is the crosstrack error, and $R_{\text {vincenty }}$ is the range computed from the inverse vincenty formulae. All heading states are measured from the north axis in NED coordinates. 
Crosstrack error is closed using a similar method as altitude, but using states defined in the lateral plane. A PD compensator on crosstrack error is used to compute the corrective heading command, $\Delta \chi_{c m d}$, needed to close the error on crosstrack.

$$
\Delta \chi_{c m d}=\left(K_{X t r a c k}+K_{d_{X t r a c k}} s\right) \text { XtrackErr }
$$

Where $K_{X t r a c k}$ is the proportional gain on crosstrack error and $K_{d_{X t r a c k}}$ is the derivative gain on the crosstrack error. The corrective heading command, $\Delta \chi_{c m d}$, is added to the runway $\chi$ command to compute the total heading command, $\chi_{\text {Backstep }}$, feeding into the heading controller. The vehicle closes on $\chi$ error generated from $\chi_{\text {Backstep }}$ and $\chi_{\text {vehicle }}$ using $\mu$ as the virtual command. These parameters are depicted in Figure 2-28.

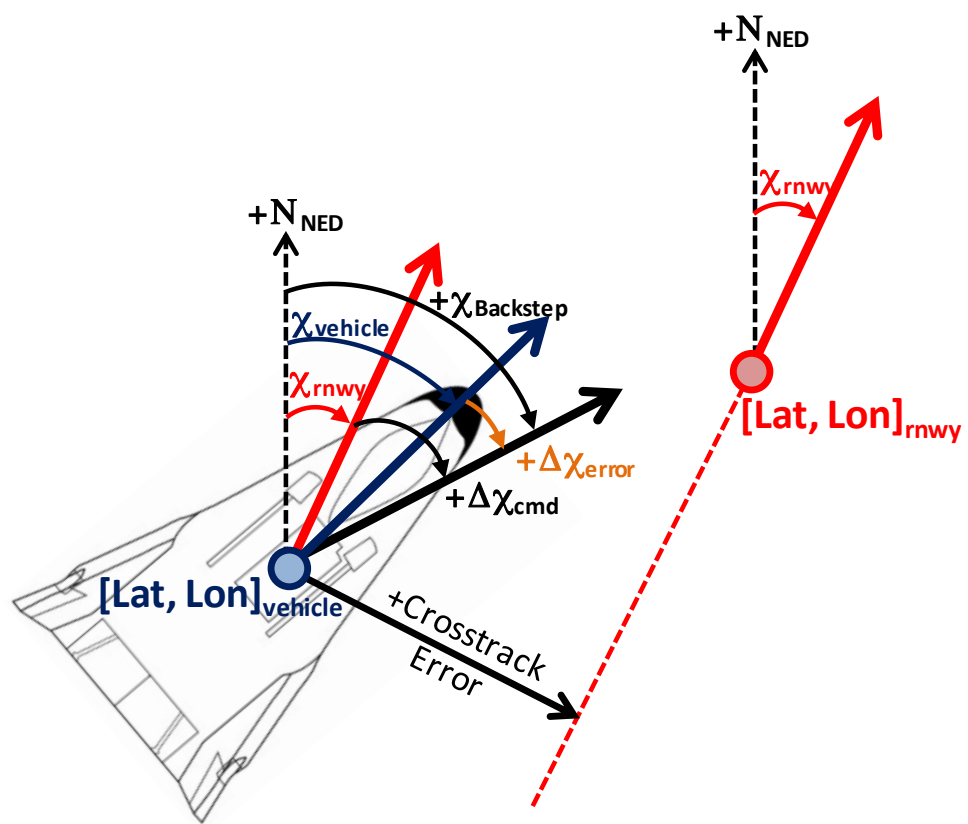

Figure 2-28: Definition of heading command developed from the crosstrack error. 
The $\chi, \mu$, and $\beta$ control laws were derived by constructing CLFs. The governing equations used for the backstepping formulation are shown in Equations (2-99) through Equation (2-101).

$$
\begin{gathered}
\dot{\chi}=\frac{L \sin \mu}{m V \cos \gamma} \\
\dot{\mu}=\frac{1}{m V}[D \sin \beta \tan \gamma \cos \mu+Y \cos \beta \tan \gamma \cos \mu+L(\tan \beta+\tan \gamma \sin \mu)] \\
-\frac{g \tan \beta \cos \gamma \cos \mu}{V}+\frac{P_{s}}{\cos \beta} \\
\dot{\beta}=\frac{1}{m V}[D \sin \beta+Y \cos \beta]-\frac{g \cos \gamma \sin \mu}{V}-R_{S}
\end{gathered}
$$

The roll rate, $P_{s}$, and yaw rate, $R_{s}$, are defined in the stability axis for the ease of putting the system of equation in strict feedback form ${ }^{41}$. However, the inner loop control law requires the roll rate and the yaw rate to be in the body axis. Body rates in stability axis are easily transformed into the body axis using the DCM in Equation (2-102) ${ }^{41}$.

$$
\left[\begin{array}{l}
P \\
R
\end{array}\right]=\left[\begin{array}{cc}
\cos \alpha & -\sin \alpha \\
\sin \alpha & \cos \alpha
\end{array}\right]\left[\begin{array}{l}
P_{s} \\
R_{S}
\end{array}\right]
$$

Equations (2-99) through (2-101) are put in strict feedback form in order to develop the backstepping control law for the roll axis. $\chi$ is represented by $x_{1}, \mu$ is represented by $x_{2}$, and $P_{s}$ is the control variable.

$$
\begin{gathered}
\dot{x}_{1}=\frac{L \sin x_{2}}{m V \cos \gamma} \\
\dot{x}_{2}=\frac{1}{m V}\left[D \sin \beta \tan \gamma \cos x_{2}+Y \cos \beta \tan \gamma \cos x_{2}+L\left(\tan \beta+\tan \gamma \sin x_{2}\right)\right] \\
-\frac{g \tan \beta \cos \gamma \cos x_{2}}{V}+\frac{u}{\cos \beta} \\
u=P_{s}
\end{gathered}
$$


Equation (2-105) $)^{41}$ can be rewritten as Equation (2-106) ${ }^{41}$ for the ease of representing all of the terms in the dynamic equation for the purpose of deriving the control law.

$$
\dot{x}_{2}=F_{\mu}\left(x_{2}, y\right)+\frac{u}{\cos \beta}
$$

where $F_{\mu}\left(x_{2}, y\right)=\frac{1}{m V}\left[D \sin \beta \tan \gamma \cos x_{2}+Y \cos \beta \tan \gamma \cos x_{2}+L\left(\tan \beta+\tan \gamma \sin x_{2}\right)\right]$

$$
-\frac{g \tan \beta \cos \gamma \cos x_{2}}{V}
$$

The influences of variables that are regarded as constants in the backstepping formulation are represented by $y$. It is assumed that a control law $\alpha_{1}$ exists as a function of $x_{1}$ so that bank angle, $x_{2}$, is used as a virtual control for heading angle, $x_{1}$.

$$
x_{2 d e s}=\alpha_{1}\left(x_{1}\right)
$$

A CLF is selected to stabilize the first subsystem represented in Equation (2-103).

$$
V_{1}=\frac{1}{2} x_{1}{ }^{2}
$$

Equation (2-110) is the time derivative of Equation (2-109) used to derive the $\alpha_{1}$ control law in Equation (2-112) to make the subsystem GAS.

$$
\dot{V}_{1}=x_{1} \dot{x}_{1}
$$

Substituting Equation (2-103) into $\dot{x}_{1}$ and substituting Equation (2-108) into $x_{2}$ :

$$
\begin{gathered}
\dot{V}_{1}=x_{1}\left(\frac{L \sin \alpha_{1}}{m V \cos \gamma}\right) \\
\alpha_{1}=\sin ^{-1}\left(\left(-k_{1} x_{1}\right) \frac{m V \cos \gamma}{L}\right) \quad \text { for } \dot{V}<0
\end{gathered}
$$

Substituting $\alpha_{1}$ into the CLF proves that the CLF is negative definite as long as $k_{l}$ is positive definite. 


$$
\begin{aligned}
& \dot{V}_{1}=-k_{1} x_{1}{ }^{2} \\
& \text { where } k_{1}>0
\end{aligned}
$$

The difference between the actual state, $x_{2}$, and the desired state obtained from the virtual control, $\alpha_{l}$ is introduced by the residual term, $z_{l}$ :

$$
\begin{aligned}
& z_{1}=x_{2}-x_{2 d e s} \\
& z_{1}=x_{2}-\alpha_{1}
\end{aligned}
$$

The system is backstepped and the residual term is included into the dynamic equations. First, Equation (2-106) is rewritten in terms of $z_{1}$. Then the dynamics of the residual term is derived by taking its derivative with respect to time.

$$
\begin{aligned}
& \dot{x}_{2}=F_{\mu}\left(z_{1}+\alpha_{1}, y\right)+\frac{u}{\cos \beta} \\
& \dot{z}_{1}=\dot{x}_{2}-\dot{\alpha}_{1} \\
& \dot{z}_{1}=F_{\mu}\left(z_{1}+\alpha_{1}, y\right)+\frac{u}{\cos \beta}-\frac{\partial \alpha_{1}}{\partial x_{1}} \dot{x}_{1}
\end{aligned}
$$

The CLF is augmented to include the residual term in order to stabilize the $\dot{x}_{1}$ and $\dot{x}_{2}$ system, shown as Equation (2-119).

$$
\dot{V}_{2}=V_{1}+\frac{1}{2} z_{1}^{2}
$$

The time derivative of the augmented CLF, in Equation (2-120) should be negative definite for GAS.

$$
\dot{V}_{2}=\dot{V}_{1}+z_{1} \dot{z}_{1}
$$


Substituting Equation (2-113) into $\dot{V}_{1}$ and Equation (2-118) into $\dot{z}_{1}$ :

$$
\dot{V}_{2}=-k_{1} x_{1}{ }^{2}+z_{1}\left(F_{\mu}\left(z_{1}+\alpha_{1}, y\right)+\frac{u}{\cos \beta}-\frac{\partial \alpha_{1}}{\partial x_{1}} \dot{x}_{1}\right)
$$

The actual control, $u$, is selected as Equation (2-122) to make the CLF negative definite.

$$
\begin{gathered}
u=\cos \beta\left(-k_{2} z_{1}-F_{\mu}\left(z_{1}+\alpha_{1}, y\right)\right) \\
\text { where } k_{2}>0
\end{gathered}
$$

In order for the CLF to be negative definite $u$ has to dominate the $\dot{\alpha}_{1}$ term, as in the backstepping derivation for the longitudinal axis. This condition is satisfied when the inner loop feedback gain is larger than the outer loop gain.

$$
k_{1}>k_{2}
$$

Using the backstepping formulation from above, the $\chi$ control law represented by the Heading Loop block in Figure 2-26 computes the $\mu$ command to close the error on $\chi$ using Equation $(2-124)$.

$$
\begin{aligned}
& \mu_{c m d}=\sin ^{-1}\left(\dot{\chi}_{\text {des }} \frac{m V \cos \gamma}{L}\right) \\
& \text { where } \dot{\chi}_{\text {des }}=k_{1}\left(\chi_{c m d}-\chi_{\text {actual }}\right)
\end{aligned}
$$

The $\mu$ control law in Equation (2-125) uses the backstepping derivation from Equation (2-122) to compute roll rate in the stability axis.

$$
\begin{aligned}
& P_{S}=\cos \beta\left(\dot{\mu}_{\text {des }}-F_{\mu}\left(z_{1}+\alpha_{1}, y\right)\right) \\
& \text { where } \dot{\mu}_{\text {des }}=k_{2}\left(\mu_{\text {cmd }}-\mu_{\text {actual }}\right)
\end{aligned}
$$

The yaw axis backstepping formulation begins by converting Equation (2-101) into pure feedback form, where $\beta$ is state, $x_{1}$, to be controlled by $R_{s}$, represented by $u$. 


$$
\begin{gathered}
\dot{x}_{1}=F_{\beta}\left(x_{1}, y\right)-u \\
\text { where } F_{\beta}\left(x_{1}, y\right)=\frac{1}{m V}\left[D \sin x_{1}+Y \cos x_{1}\right]-\frac{g \cos \gamma \sin \mu}{V} \\
u=R_{S}
\end{gathered}
$$

Like the short hand equation for $\mu$ dynamics, $y$ represents the variables that are considered constants in the backstepping formulation.

A CLF is chosen to stabilize the $x_{1}$ system.

$$
V_{1}=\frac{1}{2} x_{1}^{2}
$$

The controller, $u$, is designed such that the time derivative of the CLF is negative definite, shown in Equation (2-129).

$$
\dot{V}_{1}=x_{1} \dot{x}_{1}
$$

Substituting Equation (2-126) for $\dot{x}_{1}$ :

$$
\dot{V}_{1}=x_{1}\left(F_{\beta}\left(x_{1}, y\right)-u\right)
$$

To make Equation negative definite:

$$
\begin{gathered}
u=-1 *\left(-k_{1} x_{1}-F_{\beta}\left(x_{1}, y\right)\right) \\
\text { where } k_{1}>0
\end{gathered}
$$

The control law for the $\beta$ feedback loop derived from Equation $(2-131)^{20}$ becomes:

$$
\begin{gathered}
R_{s}=-\dot{\beta}_{\text {des }}+F_{\beta}\left(x_{1}, y\right) \\
\text { where } \dot{\beta}_{\text {des }}=k_{1}\left(\beta_{c m d}-\beta_{\text {actual }}\right)
\end{gathered}
$$


The roll and yaw in the stability axis computed from Equations (2-125) and (2-131) has to be transformed in body coordinates using Equation (2-102) in order to put the body rate commands in the same coordinate frame as the dynamic inversion controller. 


\subsubsection{Lateral-Directional Axis Backstepping Implementation into Simulink}

Figure 2-29 depicts the Simulink implementation of the lateral-directional axis backstepping controller.

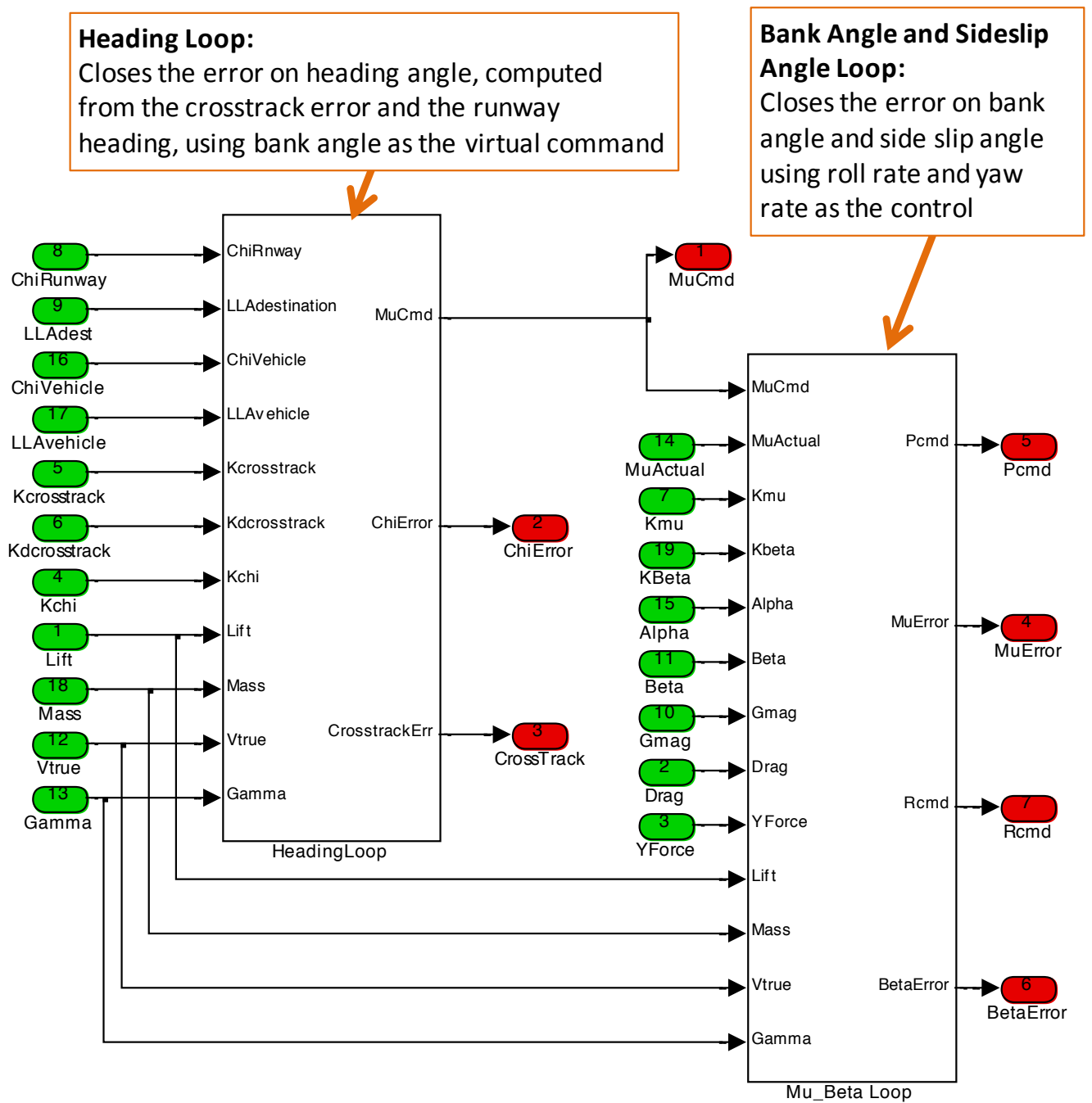

Figure 2-29: Lateral-directional axis backstepping controller implementation.

The $\chi$ loop corrects for crosstrack and $\chi$ command. A PD controller on crosstrack error,

calculated from Equation (2-97), generates a corrective $\chi$ command. The corrective $\chi$ command and the runway $\chi$ command are summed up and used as the total $\chi$ command for the vehicle to 
track using the control law in Equation (2-124). Figure 2-30 depicts these loop closures inside the Heading Loop block from Figure 2-29.

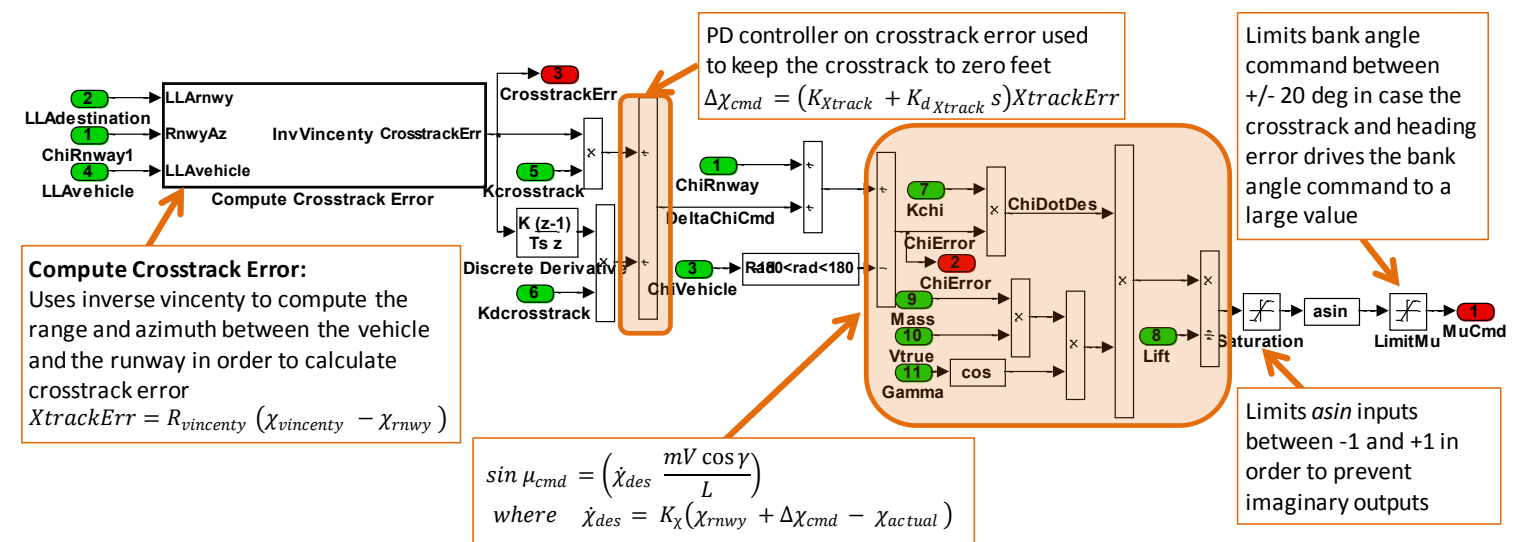

Figure 2-30: Heading loop implementation into Simulink.

The $\mu$ command computed from the control law in Equation (2-124) is limited between $+/-20$

degrees. This will guarantee that $\mu$ command will remain within values where the RLV can still maintain control and maneuver in the pitch axis.

The bank angle and sideslip angle control laws from Equation (2-125) and (2-132) are computed from using an Embedded Matlab block as seen in Figure 2-31. $\mu$ and $\beta$ are fed back to calculate errors that are operated on by the proportional gains to obtain $\mu$ and $\beta$ acceleration. Appendix $\mathrm{C}$ contains functions used to compute the $\mathrm{P}$ and $\mathrm{R}$ commands from the desired $\mu$ and $\beta$ acceleration. 


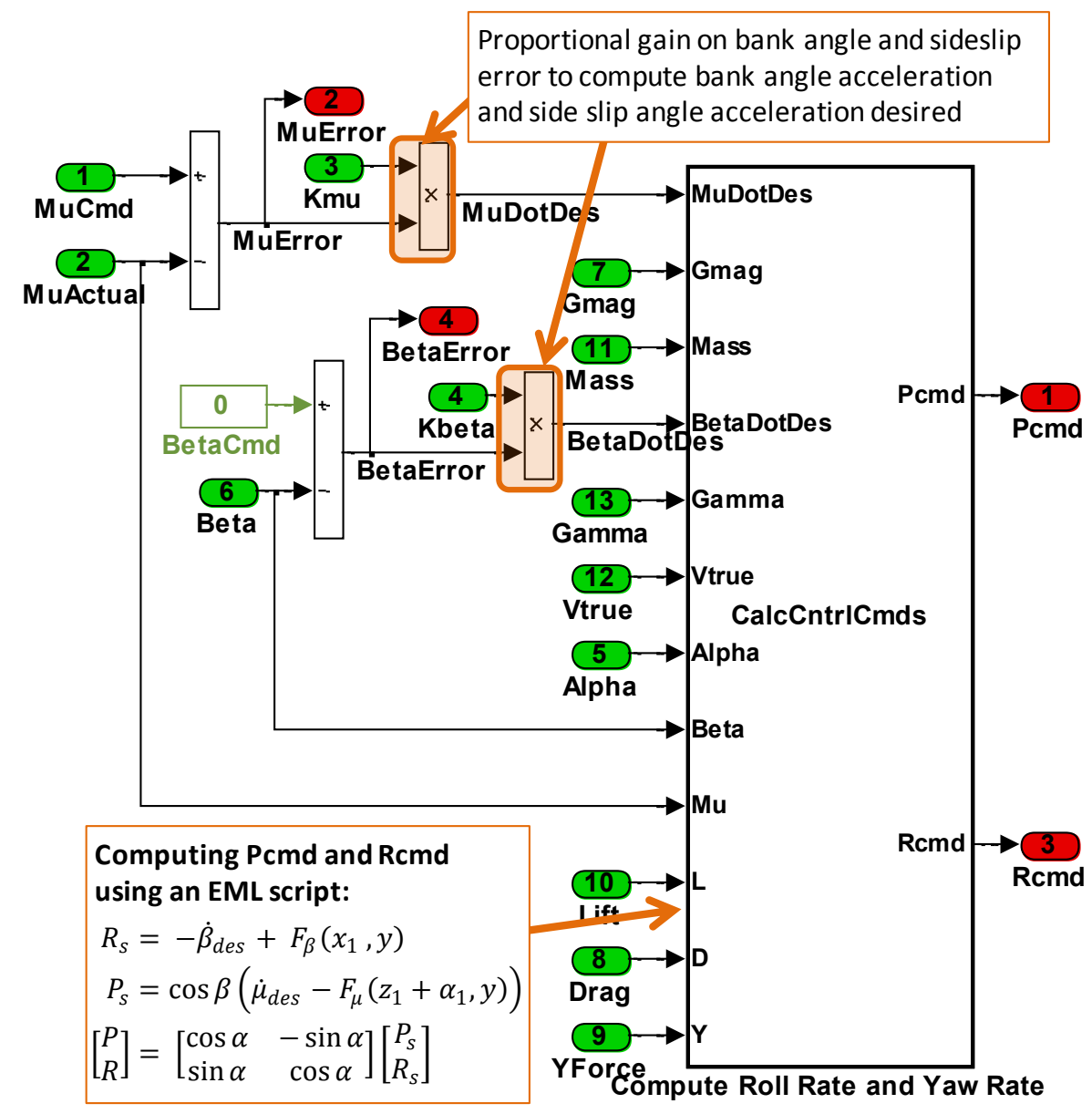

Figure 2-31: Bank angle and side slip angle control law implementation.

\subsubsection{Backstepping Controller Validation and Performance}

\subsubsection{Longitudinal-Axis Backstepping Controller Validation}

The longitudinal-axis backstepping controller performance is validated by ensuring that the altitude tracking error and $\alpha$ tracking error are within the specified limits for good tracking in the figures of merit. Altitude error will have to be within $+/-10$ feet and $\alpha$ maximum steady state error has to be within $+/-10 \%$. Each performance metric is tested one loop closure at a time to 
verify proper gains are chosen. The gains are selected for the baseline controller, which has no aerodynamic modeling error between the plant model and the on-board controller model.

The loop closure is performed from the inner-most loop to the outer loop, starting with the $\alpha$ loop first. An $\alpha$ doublet test case is performed in order to verify gains are valid for both positive and negative maneuvers. The altitude and $\gamma$ feedback loops are broken, only allowing the $\alpha$ loop to function. While an $\alpha$ doublet is commanded, $\mu$ and $\beta$ of zero degrees are commanded. The flight condition is frozen at the approach and landing interface, described in Table 1-9. The flight condition is frozen in order to maintain the RLV's energy during a doublet maneuver when the vehicle is in an unpowered configuration. Figure 2-32 shows the $\alpha$ response to a doublet command for the chosen $\alpha$ gain. The closed loop response looks like a first order system by utilizing a proportional gain to close the error on $\alpha$ without a derivative or an integral gain. A proportional gain of 0.9 is selected. The maximum absolute steady state error is less than $3.37 \%$, indicating good tracking performance.

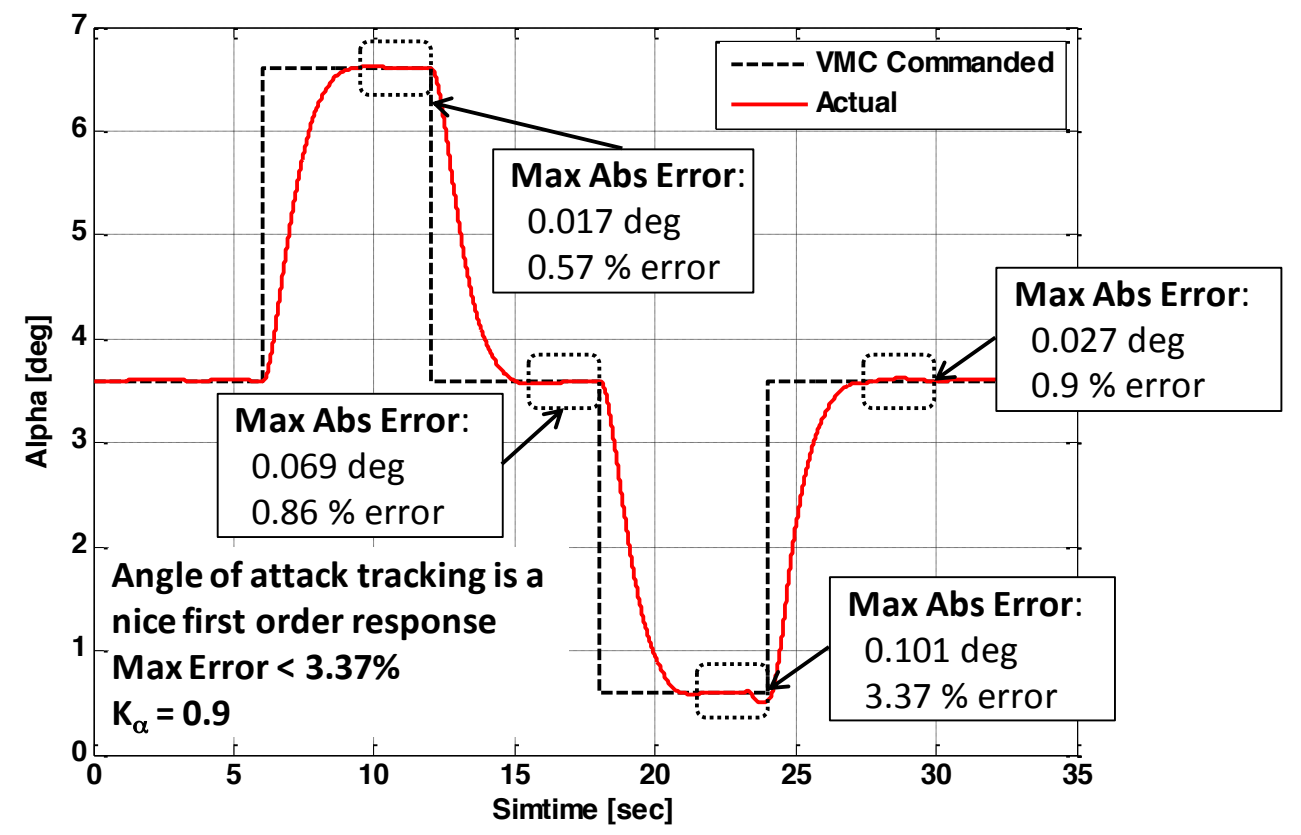

Figure 2-32: Angle of attack tracking using the backstepping controller. 
The next loop closure is on $\gamma$. The $\alpha$ loop is kept intact with the selected gain from the $\alpha$ doublet test case. The flight condition can no longer be frozen for the $\gamma$ doublet because the vehicle has to be free to move in the vertical plane. Therefore, the acceleration and velocity vectors that were previously zeroed out are allowed to propagate again. The altitude loop is broken, while the $\gamma$ and the $\alpha$ loops are closed. A $\gamma$ doublet is conducted from the approach and landing interface condition, specified in Table 1-6, to test both positive and negative maneuvers like the $\alpha$ test case. The backstepping formulation requires a $\gamma$ acceleration command, which is zeroed out for this test case since a step input is discontinuous, which will make the acceleration term go to infinity and back down to zero for each step command. The crosstrack and $\chi$ feedback loops are broken since they are not of a concern when testing the longitudinal-axis controller. $\mu$ and $\beta$ are commanded to be zero degrees. It is desired to have the least amount of interference from the lateral plane. Figure 2-33 depicts the $\gamma$ doublet tracking with the subsequent $\alpha$ performance below. An integral gain of 0.008 and a proportional gain of 0.25 are selected for the loop closure. The $\gamma$ dynamics are used to compute the $\alpha$ necessary to close the loop on $\gamma$. Therefore, $\alpha$ tracking error is proportional to $\gamma$ tracking. $\alpha$ has a first order closed loop response, which means that for a ramp input there will always be a steady state error. As long as $\alpha$ tracking has an error then the $\gamma$ tracking has an error, as demonstrated in Figure 2-33. 

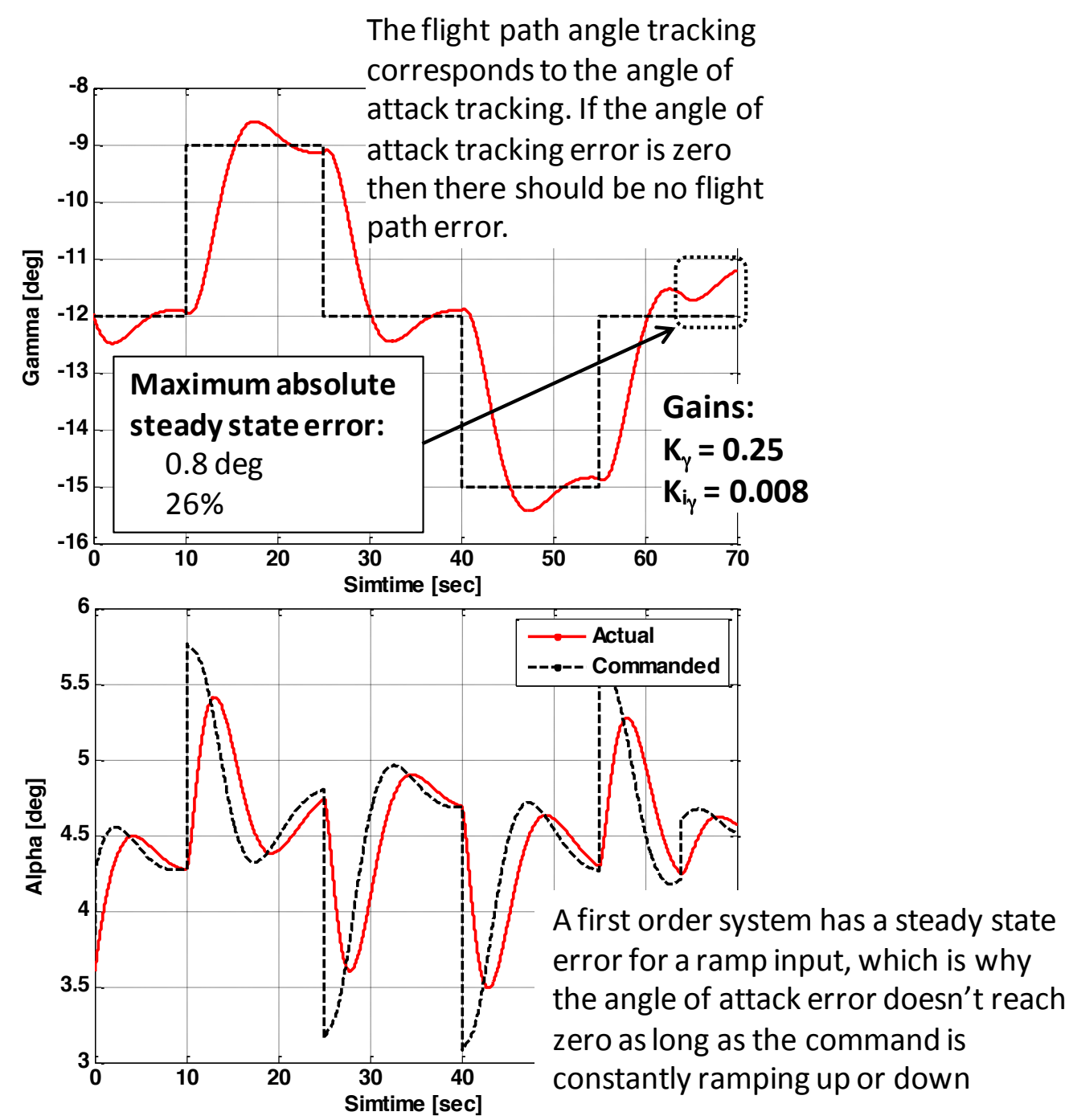

Figure 2-33: Flight path angle doublet command response and the associated angle of attack response.

A $\gamma$ capture is also performed to check $\gamma$ response since the doublet response was hard to evaluate tracking when the $\alpha$ command is oscillating. Figure 2-34 shows results for a positive step input of 3 deg commanded for $\gamma$. The maximum absolute steady state error is about $8 \%$ which is only $0.242 \mathrm{deg}$ error, which seems like a valid response. 

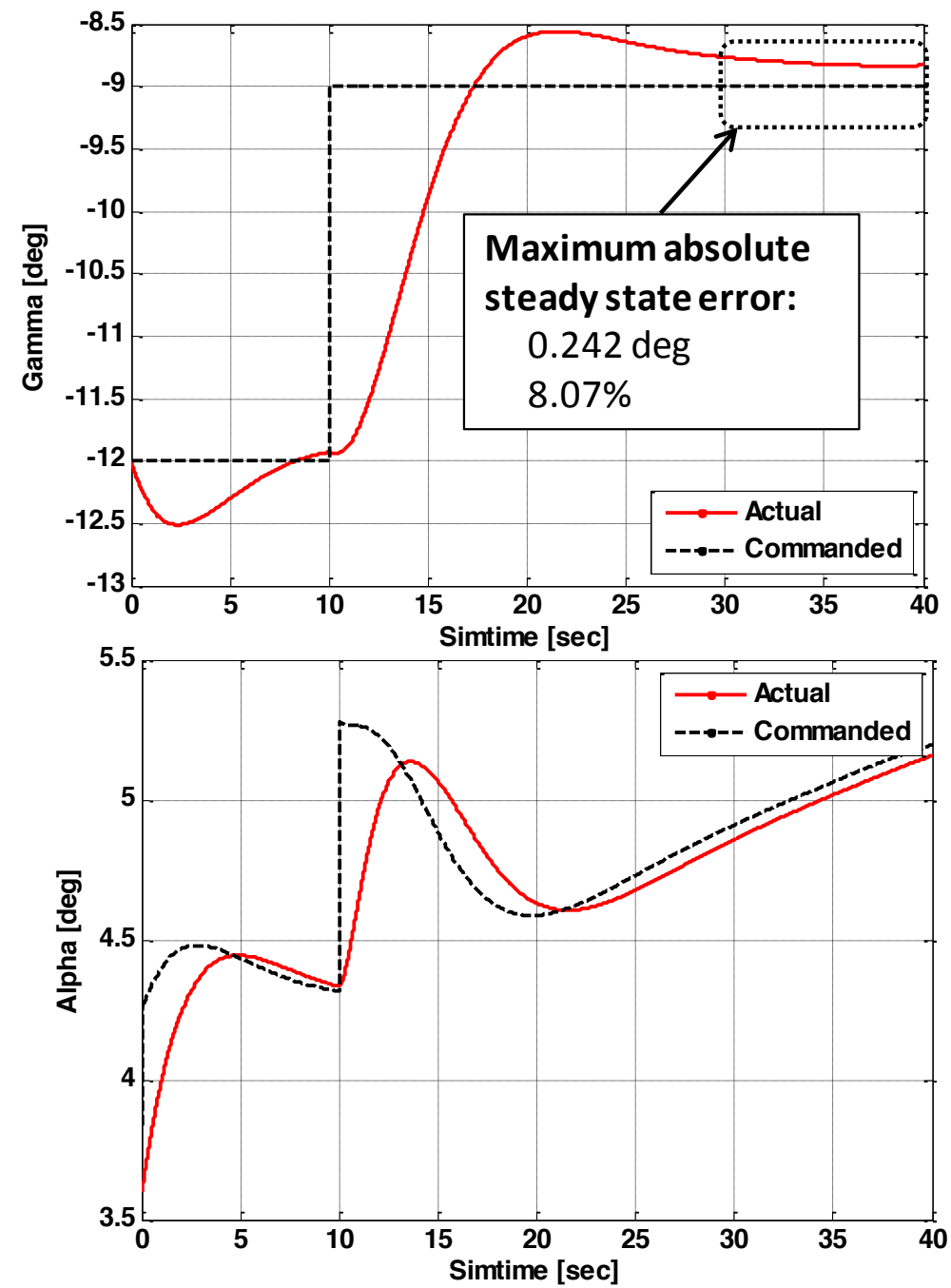

Figure 2-34: Flight path angle capture test case with a positive step input command.

The outer most loop closure on altitude is tested after $\gamma$ and $\alpha$ feedback loops are validated. The first 60 seconds of the approach and landing trajectory commands a constant steep glide slope corresponding to a $\gamma$ acceleration command of zero deg/sec and a constant sink rate. This means that the altitude command will be a negative ramp. A step altitude command cannot be performed since the vehicle is unpowered and only has a limited amount of energy. Once again, the crosstrack and $\chi$ feedback loops are broken and $\mu$ and $\beta$ are commanded to zero in order to limit the amount of control power used for the lateral direction. Figure 2-35 shows the 
altitude response for an integral gain of 0.25 and a proportional gain of 0.05 and a derivative gain of 1.7. The vehicle is not exactly trimmed at the initial condition which causes a transient response in $\gamma$ tracking initially. Altitude error generates from the vehicle not achieving the proper $\gamma$ in the first 12 seconds of the simulation. However, once $\gamma$ error decreases, so does the altitude error for the last 45 seconds. The integral term on altitude error allows the error to decrease even if there is an error on $\gamma$. Maximum absolute error on altitude, including the transient response, is within 6.2 feet which validates gains selected for the altitude and $\gamma$ loop since the controller exhibits good tracking according to the figures of merit. 

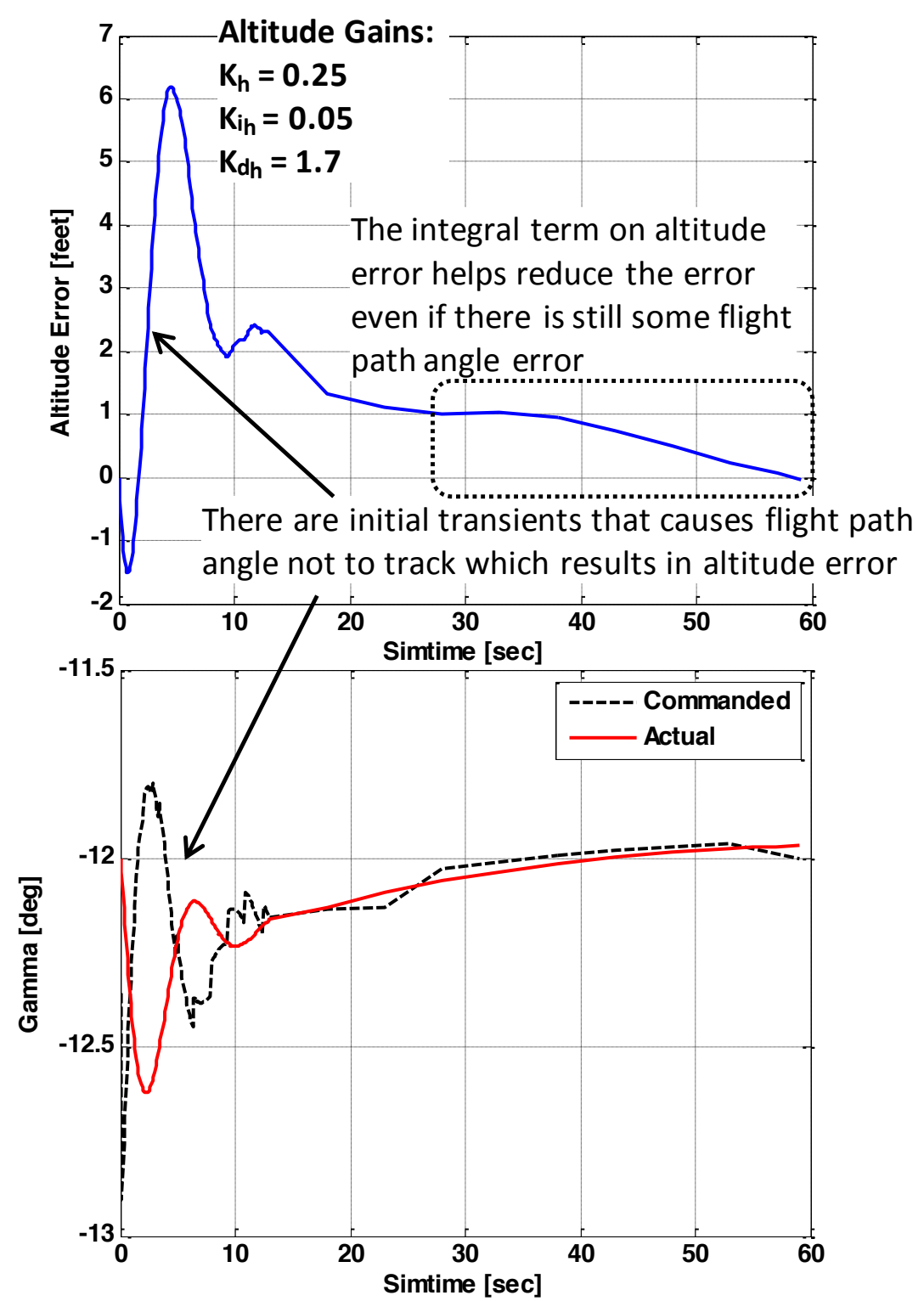

Figure 2-35: Altitude tracking for a ramp input with the corresponding flight path angle tracking.

\subsubsection{Lateral-Directional Axis Backstepping Controller Validation}

The lateral-directional axis controller is validated in a similar fashion as the longitudinalaxis controller. Each feedback loop will be closed one at a time to verify that selected gains will provide good tracking performance. The gains are chosen for the baseline controller, which does 
not have any unmodeled aerodynamic uncertainty in the on-board aerodynamic model. Figures of merit for the lateral-directional axis controller performance are to keep the crosstrack error within $+/-5$ feet and keep $\mu$ steady state error within $+/-10 \%$.

The inner most loop of the lateral-directional axis is the feedback on $\mu$ and $\beta$. There is no need to perform a doublet on $\beta$ since a non-zero $\beta$ is never desired for the RLV. Therefore, a $\mu$ doublet test case is conducted, while keeping $\alpha$ and $\beta$ command to zero degrees. Like $\alpha$ tracking verification, the flight condition is frozen at the approach and landing interface in order to retain the vehicle's energy. Figure 2-36 shows results from this test case using a proportional gain of 0.95 on $\mu$ feedback loop and a proportional gain of 0.7 on $\beta$ feedback loop. The $\mu$ control law computes the proper roll rate command to achieve the $\mu$ command using knowledge of vehicle dynamics. Therefore, if the vehicle can track roll rate command without error, then the vehicle can track its $\mu$. The first $\mu$ step command at 6 seconds causes control surfaces to saturate. Previous inner loop validations from Section 2.1.7.2 show that positive roll rate acceleration may cause the control surfaces to saturate at the approach and landing interface flight condition. The reason being that not enough control power exists to provide positive pitch moment to cancel inherent plant dynamics for that flight condition and still provide positive roll acceleration for the desired dynamics. The same non-minimum phase zero behavior in roll rate is seen $\mu$. When control effectors do not saturate, the vehicle has no problem tracking the roll rate command from the $\mu$ control loop. When the roll rate error goes to zero the $\mu$ error approaches zero degree as well. The maximum absolute $\mu$ steady state error is within $8.5 \%$, which is good tracking performance according to the figures of merit. 

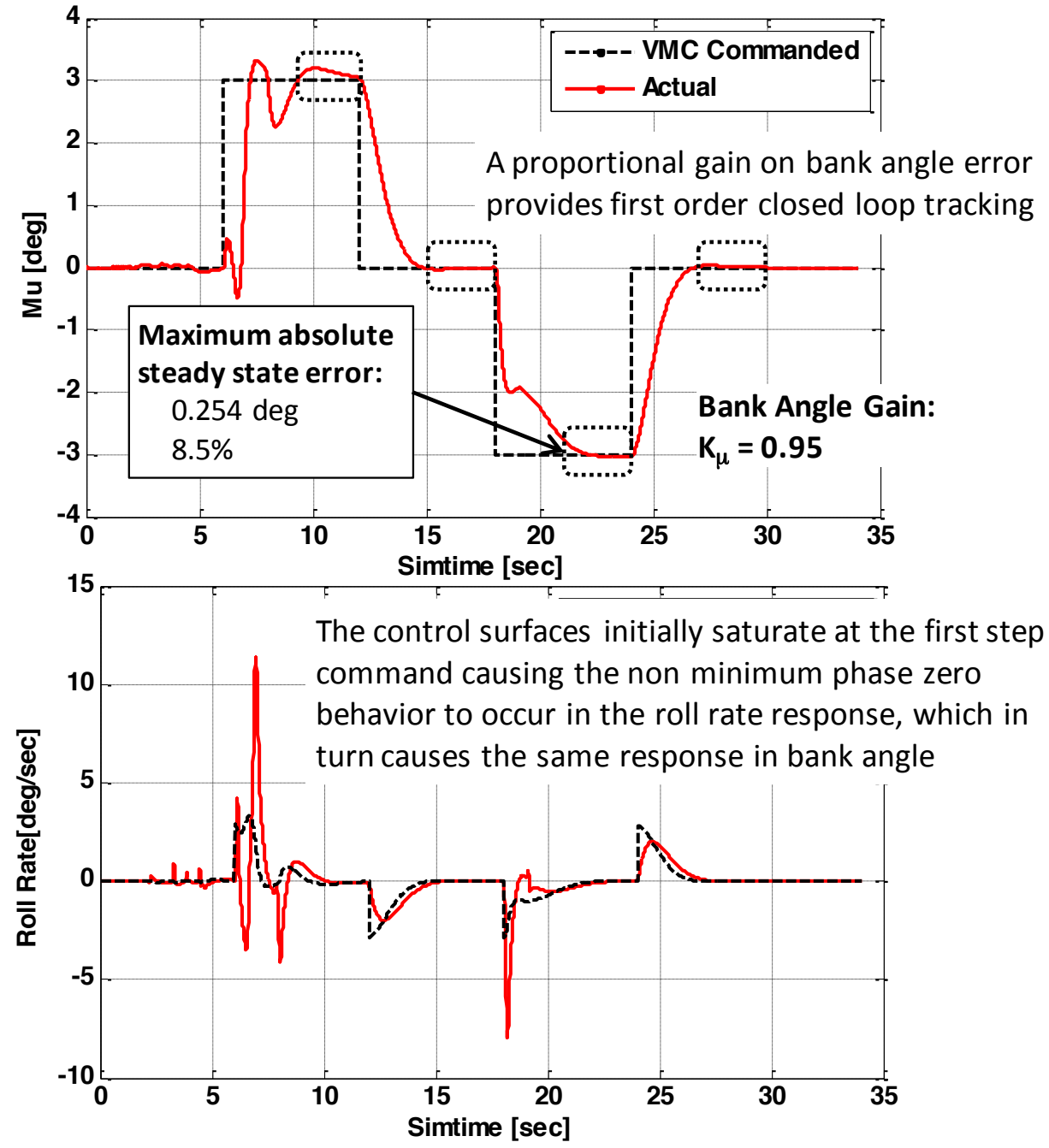

Figure 2-36: Bank angle doublet response and the corresponding roll rate response.

Figure 2-37 depicts the $\beta$ response to a command of zero degrees while the $\mu$ doublet is performed. The bank angle maneuver induces a yaw rate which creates a non-zero $\beta$. The controller tries to reduce the amount of $\beta$ deviation when performing the $\mu$ maneuver, as seen in Figure 2-37. $\beta$ is within $+/-0.3$ degrees, which is small enough to keep drag forces and side forces to a minimum, preventing negative control effects on the vehicle. 


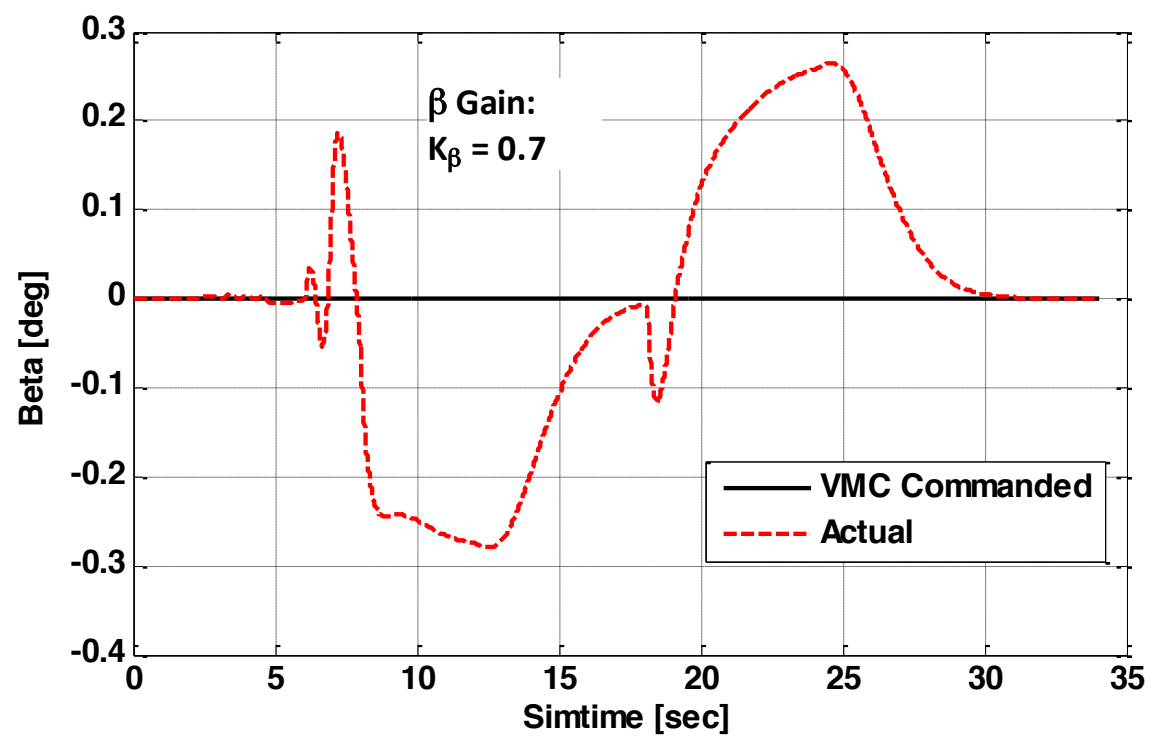

Figure 2-37: The side slip angle during the bank angle doublet maneuver.

The $\chi$ feedback loop is closed once proper gains are set for $\beta$ and $\mu$ loops. A doublet is used as the $\chi$ command to evaluate controller performance with the selected gain. In order to change $\chi$ angles, the vehicle has to be able to maneuver in the lateral plane, which means that the flight condition cannot be frozen like it was for the $\mu$ doublet. Therefore, the acceleration and velocity integral terms are allowed to propagate from the approach and landing interface condition from Table 1-6. The crosstrack loop, the altitude loop, and the $\gamma$ loop remain broken so that the vehicle only responds to $\chi, \mu, \beta$, and $\alpha$ error. A constant $\alpha$ command of 6.6 degrees is fed into the $\alpha$ controller, while altitude and $\gamma$ propagates freely. This will allow minimum interference from the longitudinal-axis controller while the lateral-directional controller is being tested. Figure 2-28 shows $\chi$ response to the doublet command. A proportional gain of 0.2 is chosen for the $\chi$ feedback loop. At this flight condition the control surfaces are close to saturation. Therefore, at the start of each step command the control surfaces saturates for a second because of the sudden increase in $\chi$ error. $\chi$ error produces a large $\mu$ acceleration command, which in turn creates a large roll acceleration command fed into the dynamic inversion. Not enough control 
power exists to be able to produce this roll acceleration as well as maintain a positive pitch acceleration to hold the angle of attack constant at 6.6 degrees. However, the vehicle is still able to maintain control and close the error on heading angle once the initial saturation occurs that changes the flight condition slightly. The maximum absolute steady state error is $0.35 \mathrm{deg}$ which is about $11.7 \%$.

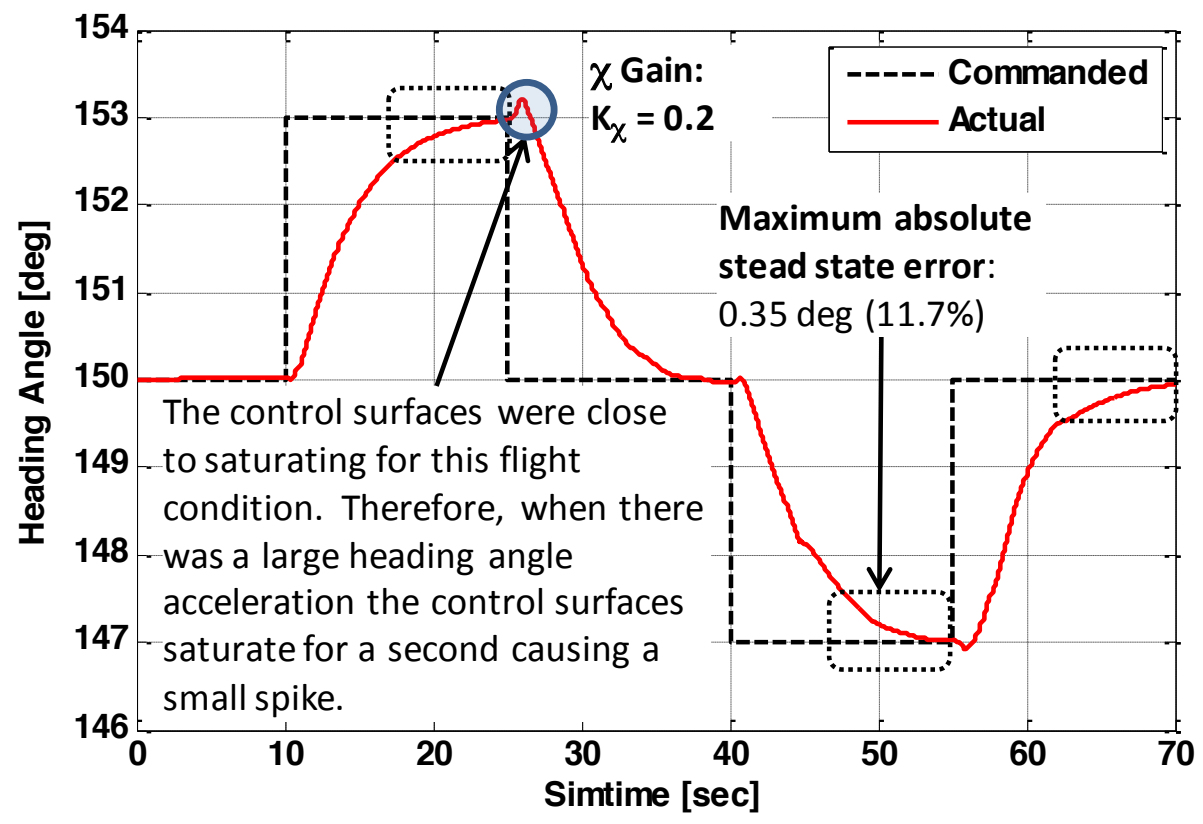

Figure 2-38: Heading angle response to a doublet command.

The objective of the approach and landing phase is to be able to land the RLV facing the runway $\chi$ as well as at the runway latitude and longitude. Following the runway $\chi$ command alone is not sufficient to land at the runway latitude and longitude. The oblateness of the earth does not guarantee that a direct $\chi$ angle from the vehicle position to the runway heading will remain constant. Therefore, closing the error on crosstrack will allow the vehicle to align its position with the runway latitude and longitude. Figure 2-39 shows how crosstrack error increases even if the vehicle heading is within $0.04 \mathrm{deg}$ of the runway $\chi$. 

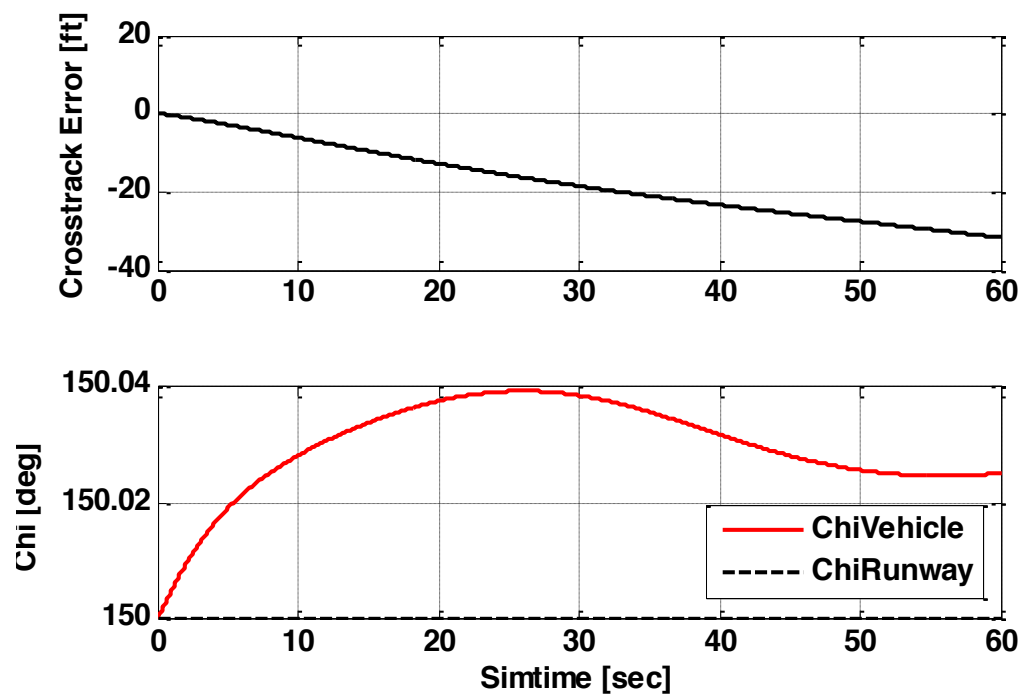

Figure 2-39: Crosstrack error increases even if the heading command tracks within 0.04 degrees.

The outer-most loop on crosstrack is validated by evaluating how well the vehicle can maintain a crosstrack error of zero. The altitude and $\gamma$ loops are broken so that a constant $\alpha$ command of $6.6 \mathrm{deg}$ is fed into the longitudinal-axis controller. The vehicle is initialized at the approach and landing interface and is allowed to propagate its velocity and position. Figure 2-40 shows the crosstrack error for a proportional gain of $0.05^{*} \mathrm{pi} / 180$ and a derivative gain of $0.095^{*} \mathrm{pi} / 180$. There are initial transients in $\chi$ since the vehicle is not initialized at an exact trim condition, which is why the crosstrack error oscillates initially. The crosstrack controller is able to keep the error within $+/-3.2$ feet even with transients, which is within the limit for good controller performance specified in the figures of merit. 


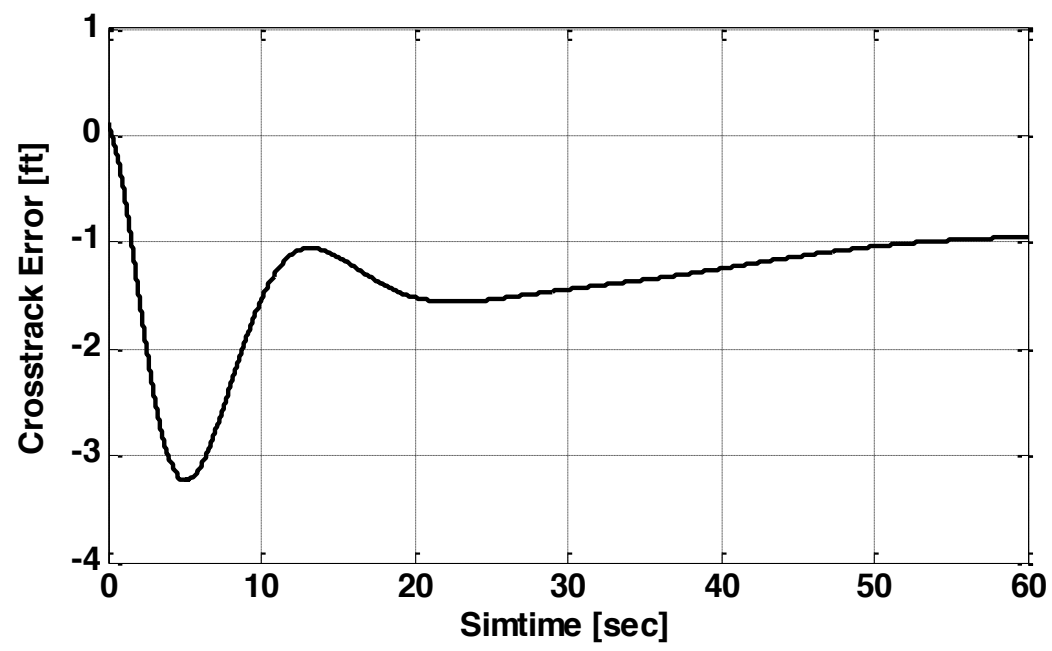

Figure 2-40: Crosstrack error after the loop closure.

\subsubsection{Backstepping Controller Performance Tracking the Landing Trajectory}

Once the longitudinal-axis backstepping controller and the lateral-directional-axis backstepping controller are validated individually, the two controllers are integrated to test its performance when tracking the approach and landing trajectory from Section 1.7.3. This will validate the baseline controller as a whole when allowing the vehicle to freely maneuver in all six axes based on the commands from guidance. Figure 2-41 through Figure 2-43 show the performance of the controller when tracking the trajectory commands. Absolute maximum altitude error, crosstrack error, and sink rate at touchdown fall within good tracking performance set by the figures of merit. 

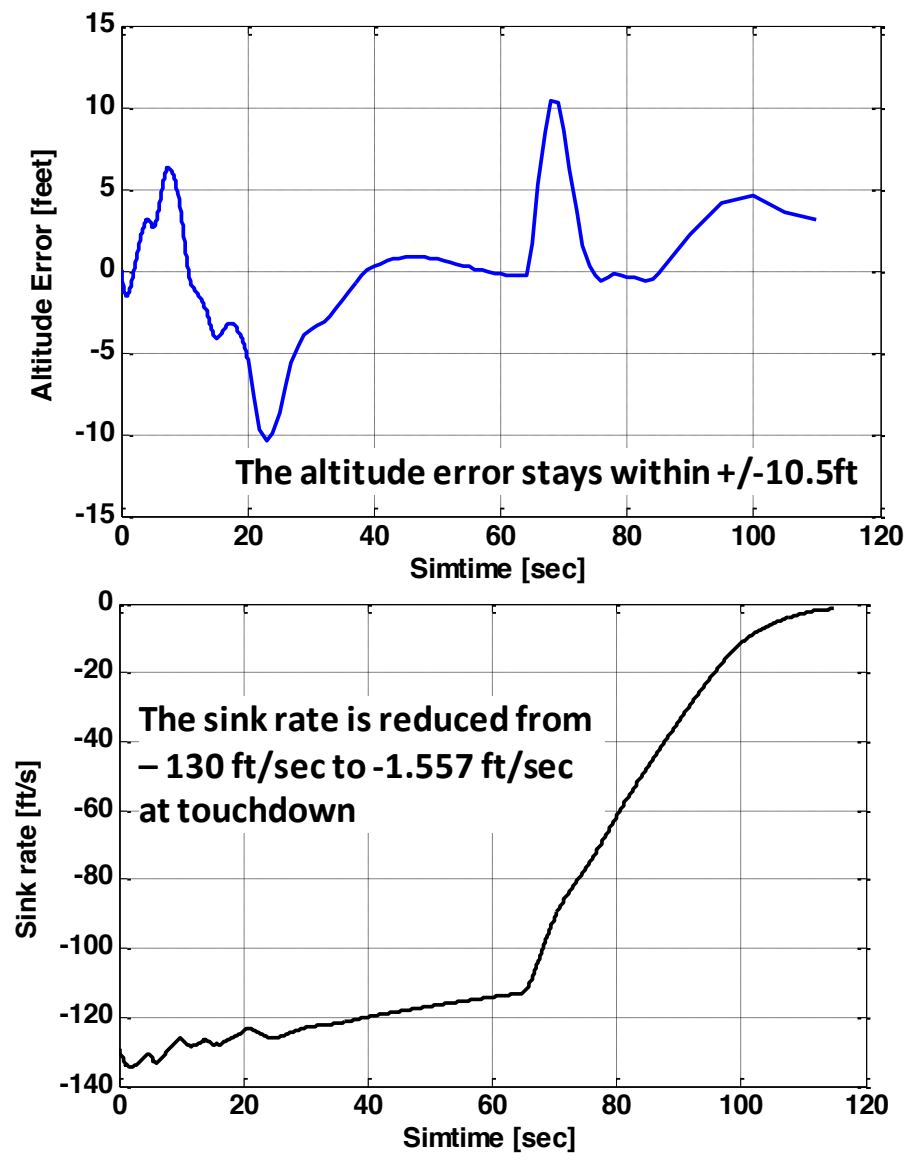

Figure 2-41: Altitude error and the sink rate for tracking the approach and landing trajectory.
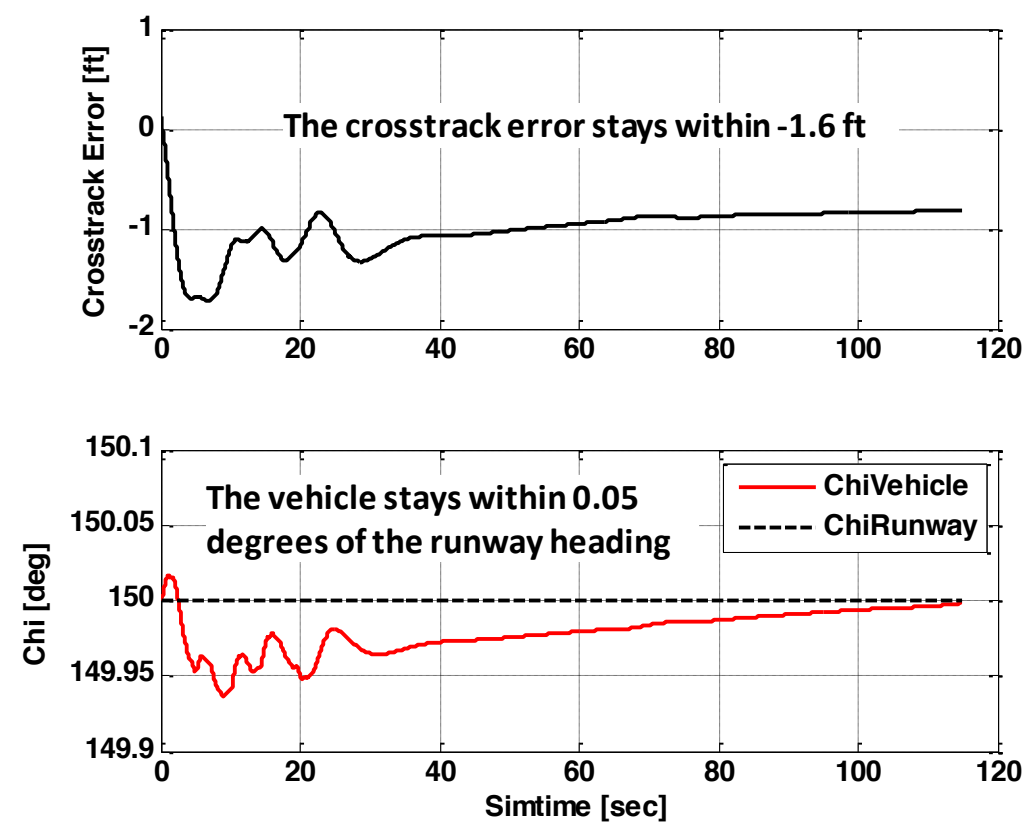

Figure 2-42: Crosstrack error and vehicle heading tracking. 
The vehicle initially experiences oscillations because the vehicle is not exactly trim at the start of the simulation. Another oscillation in $\alpha$ is induced when a large $\gamma$ acceleration is commanded. However, after the vehicle settles down it is able to track its $\gamma$ and $\alpha$ commands well.

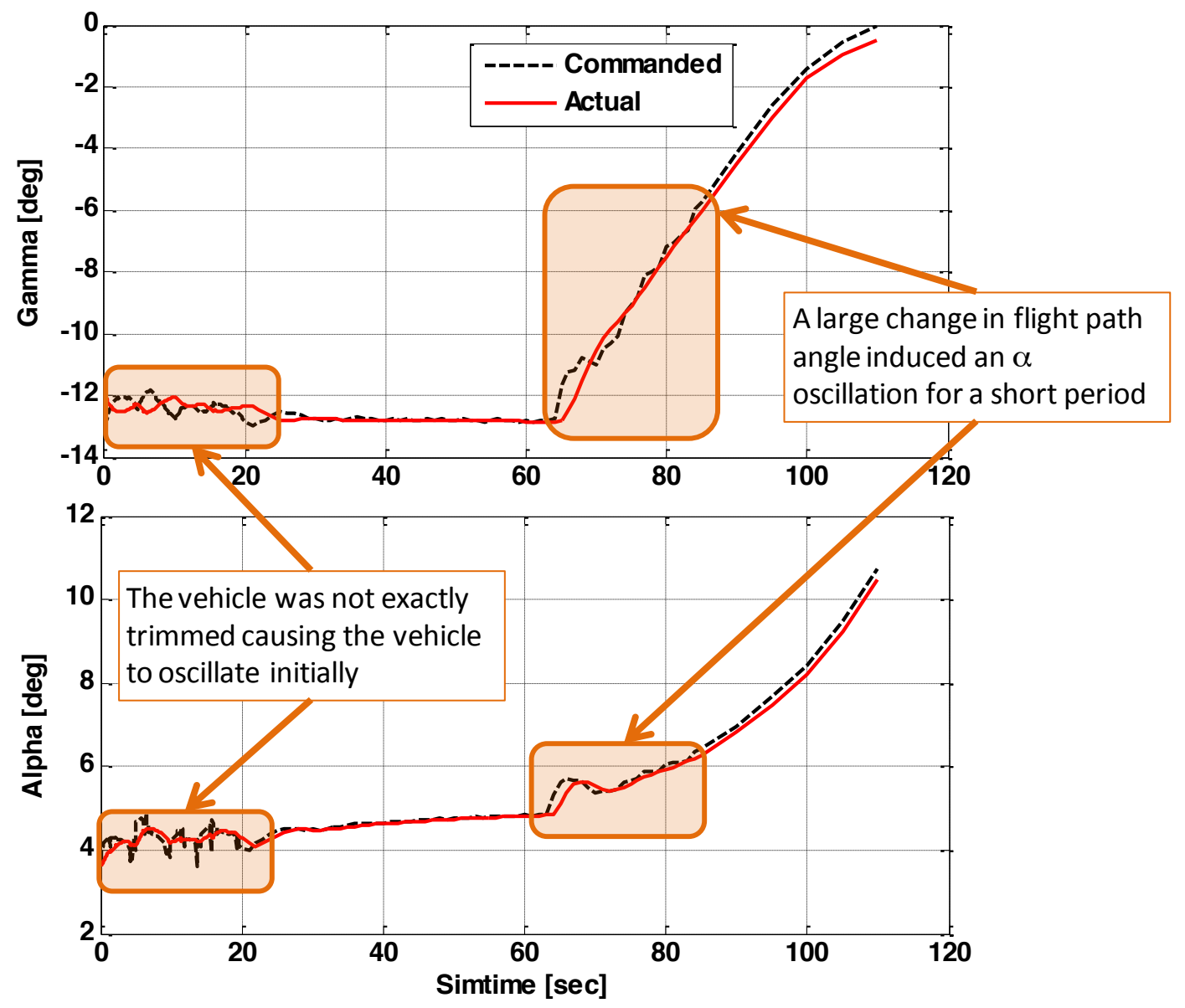

Figure 2-43: Flight path angle and angle of attack tracking during the approach and landing phase.

The oscillations at the start of the simulation are worse than the oscillations induced by the $\gamma$ command. The reason is because the dynamic inversion controller is unable to track the body rate commands as well, shown in Figure 2-44. 


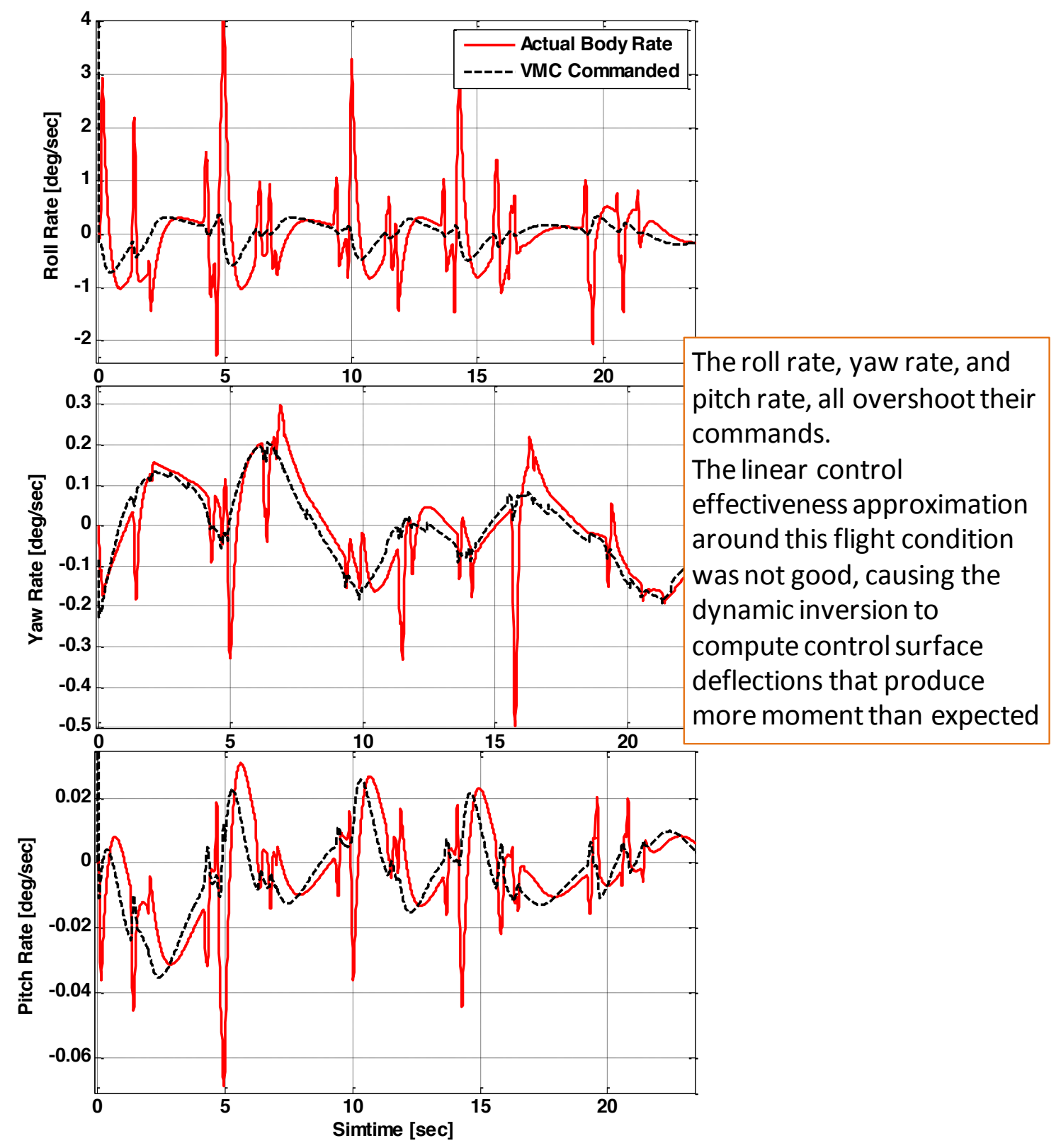

Figure 2-44: Body rate response for the first 25 seconds of the trajectory.

The body rate responses overshoot their commands for the first 23 seconds of the simulation. This is because the local control derivative approximation used to solve for the control deflection is not a good linear approximation. Moreover, the control effectiveness matrix is close to singularity at times causing the controller to command large control deflections which causes sudden spikes in body rate. The error builds up in the integral term that also contributes to 
the overshoot whenever there is a sudden change in body rate commands. After 25 seconds, the vehicle approaches a flight condition that allows the dynamic inversion to solve for the control deflection without much error. The outer loop commands like $\alpha, \mu, \chi$, and $\gamma$ can track commands better once the inner loop is able to track its command without much overshoot. Regardless of the initial transients, the baseline controller is able to provide good tracking of the approach and landing trajectory. 


\section{Robustness Analysis}

The baseline controller consists of the dynamic inversion inner loop controller and a backstepping outer loop controller. The dynamic inversion requires knowledge of static and control surface aerodynamics in order to cancel inherent rotational dynamics caused by a bare airframe, and replace them with desired body rate dynamics using moments from control surface deflections. The backstepping controller also utilizes static and control aerodynamic models to compute proper flight path commands in order to close the error on altitude and crosstrack.

The baseline controller is broken up into smaller subsystems when CLFs are formulated to prove GAS. The dynamic inversion controller proved to be GAS using only the rotation dynamic states (roll, pitch, and yaw rate). The CLF assumes the control effectiveness matrix is linear and invertible to solve for the control deflections, which is a simplification to a linear approximation that is actually implemented. The backstepping controller separates into longitudinal-axis and lateral-directional axis in order to derive control laws use CLFs. Assumptions for both formulations does not include states from the dynamic inversion controller or the rotational dynamics. Simplified 3 DoF equations are used to formulate the CLF for the longitudinal-axis controller, assuming that $\beta$ is constantly zero degrees. Moreover, it is assumed that the vehicle's lift curve slope is a linear function, when in fact it has to be linearized in a similar fashion as the control surface effectiveness in the dynamic inversion control allocator formulation. The lateral-directional axis controller derivation does not include rotational dynamics or the dynamic inversion controller states as well. These assumptions help simplify the dynamics of the vehicle in order to use CLFs to obtain control laws that are GAS stable for that simplified system. 
Both the dynamic inversion and the backstepping controller are model-based controllers that require knowledge of plant aerodynamics. The controller performance may be affected by aerodynamic modeling error. Even if parts of the system are proven to be GAS using CLFs, the derivations are highly simplified and did not include all vehicle states in the whole system. All states will need to be included in a CLF in order to prove an entire system is GAS. The robustness study helps quantify whether some of the robustness characteristics are retained when a controller integrates control laws that are proven to be GAS individually.

As mentioned in Section 1.9, static and control surface aerodynamic modeling uncertainties are inserted into the plant model to see how well the controller performs. The following sections provides the robustness analysis on body rate doublet test cases and trajectory following test cases for various aerodynamic modeling errors between the controller and the plant. Four test cases are analyzed with static aerodynamic modeling uncertainty, then control surface aerodynamic modeling uncertainty and then both modeling uncertainties together. This analysis helps determine which type of aerodynamic data affects the controller performance; and therefore, shall be more accurately modeled if this controller is used. The test matrix will be used to quantify how robust the controller will be for different types of aerodynamic uncertainties.

\subsection{Body Rate Doublet Robustness Analysis}

Body rate doublets were conducted as described in Section 1.9.2.1 by freezing the flight condition at the approach and landing interface. A doublet in one axis is conducted while commanding zero deg/sec for the two axes, which is repeated for all three axes. Commanding zero $\mathrm{deg} / \mathrm{sec}$ in other axes prevents minimal interference from the two axes. The figures of merit 
in Section 1.10 qualifies good controller performance as having less than $5 \%$ absolute steady state error, acceptable as having less than or equal to $10 \%$ absolute steady state error, and poor performance as having $20 \%$ or more absolute steady state error.

The body rate doublet test cases analyze robustness characteristics of the dynamic inversion controller. This section will particularly focus on how different types of aerodynamic modeling errors, static and control surface aerodynamic data, affect the controller performance. The first subset of body rate doublet tests looks at the roll performance using a dynamic inversion controller as aerodynamic uncertainties are increased for both static aerodynamics and control surface aerodynamics, individually, and together.

Figure 3-1 demonstrates the dynamic inversion controller tracking a roll rate doublet with $30 \%$ uncertainty in static aerodynamics, control surface aerodynamics, and both for 300 simulation runs. An average and a standard deviation are taken for a set of 300 simulation runs, and plotted on top of the original controller responses. 

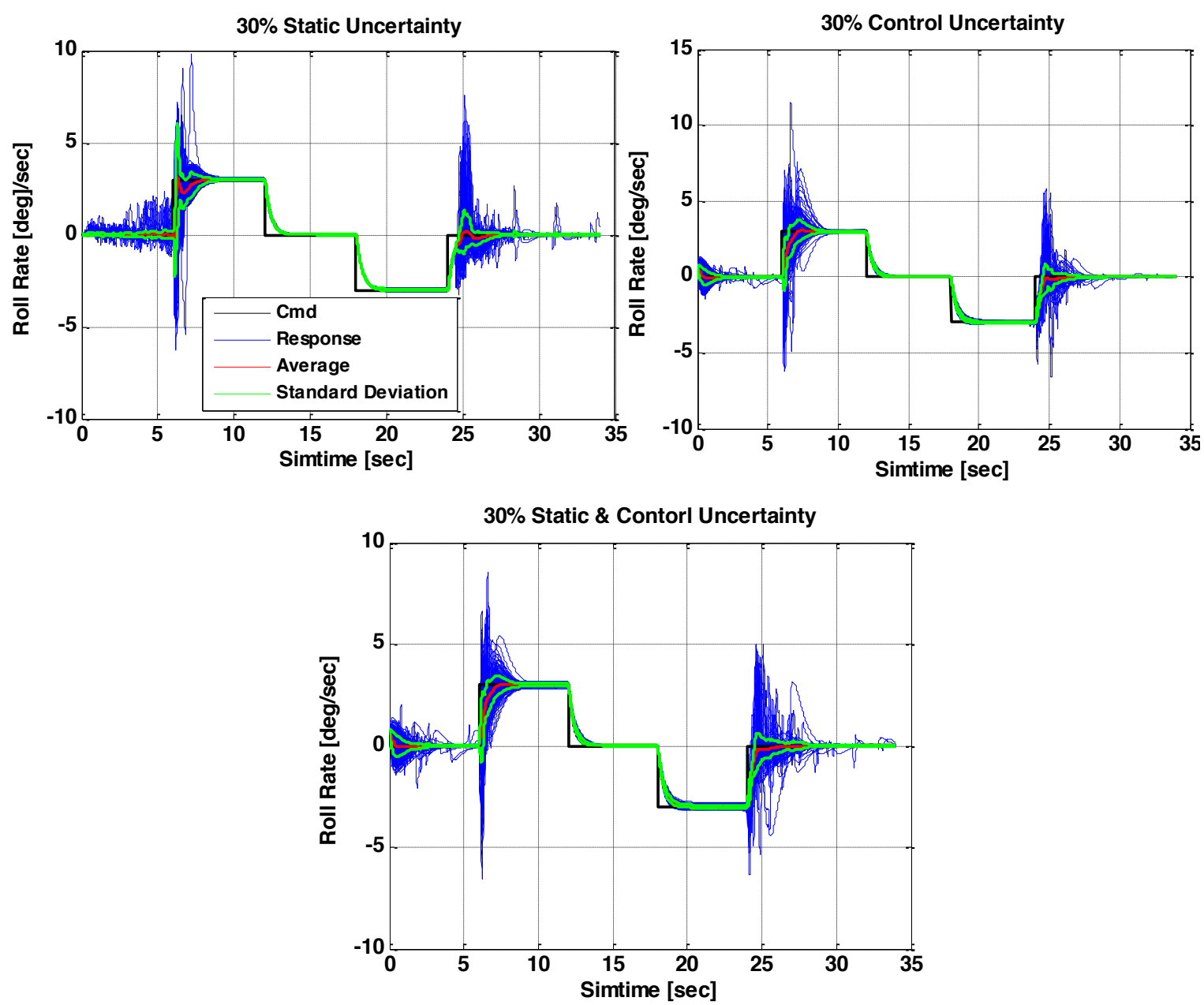

Figure 3-1: Roll rate response for $30 \%$ plant uncertainty.

For all three uncertainty cases, the controller is able to perform negative roll acceleration maneuvers without saturating control surfaces, much like the baseline performance. The integral term in the prefilter is able to close the error on roll rate in presence of plant uncertainties. However, the roll axis saturates for positive acceleration maneuvers causing non-minimum phase zero to appear from inexact plant cancellation from the control effectors, as seen in the baseline performance. The flight condition requires positive pitch acceleration to hold a zero deg/sec pitch rate, so additional positive roll acceleration causes control surface saturations since there is not enough control power to provide positive acceleration in both axes. Each control effector has limited amount of control power, thus, if all control authority is given to the pitch axis there is no 
control power available to the roll axis. Control saturation prevents the dynamic inversion from cancelling inherent plant dynamics causing undesirable non-minimum phase zero behavior of large undershoots and overshoots before settling to a steady state value.

All uncertainty cases oscillate initially in the first 5 seconds from not properly trimming the vehicle because of errors introduced from plant uncertainties. The oscillations are larger in magnitude and more frequent for the static aerodynamic uncertainty case since moment contributions from static terms are larger than moment contributions from control surface terms. The static aerodynamic uncertainty terms cause larger errors in desired acceleration computation. For some simulation runs, the dynamic inversion controller computes positive desired roll acceleration in order to correct for trimming error. As mentioned previously, the vehicle already requires positive pitch acceleration so additional positive roll acceleration results in control surface saturations generating spikes in roll response until less roll acceleration is commanded.

Figure 3-2 compares the standard deviations for each uncertainty case from Figure 3-1. The static uncertainty case follows the baseline results more closely exhibiting non-minimum phase zero. Control uncertainty case shows minimal undershoot and overshoot for positive roll acceleration. Some of the control uncertainty simulation runs produce positive pitch and roll acceleration without saturating control surfaces because the uncertainty error increased control effectiveness in the plant. Therefore, in some cases, control uncertainty helps improve the controller performance by eliminating axis saturation. 


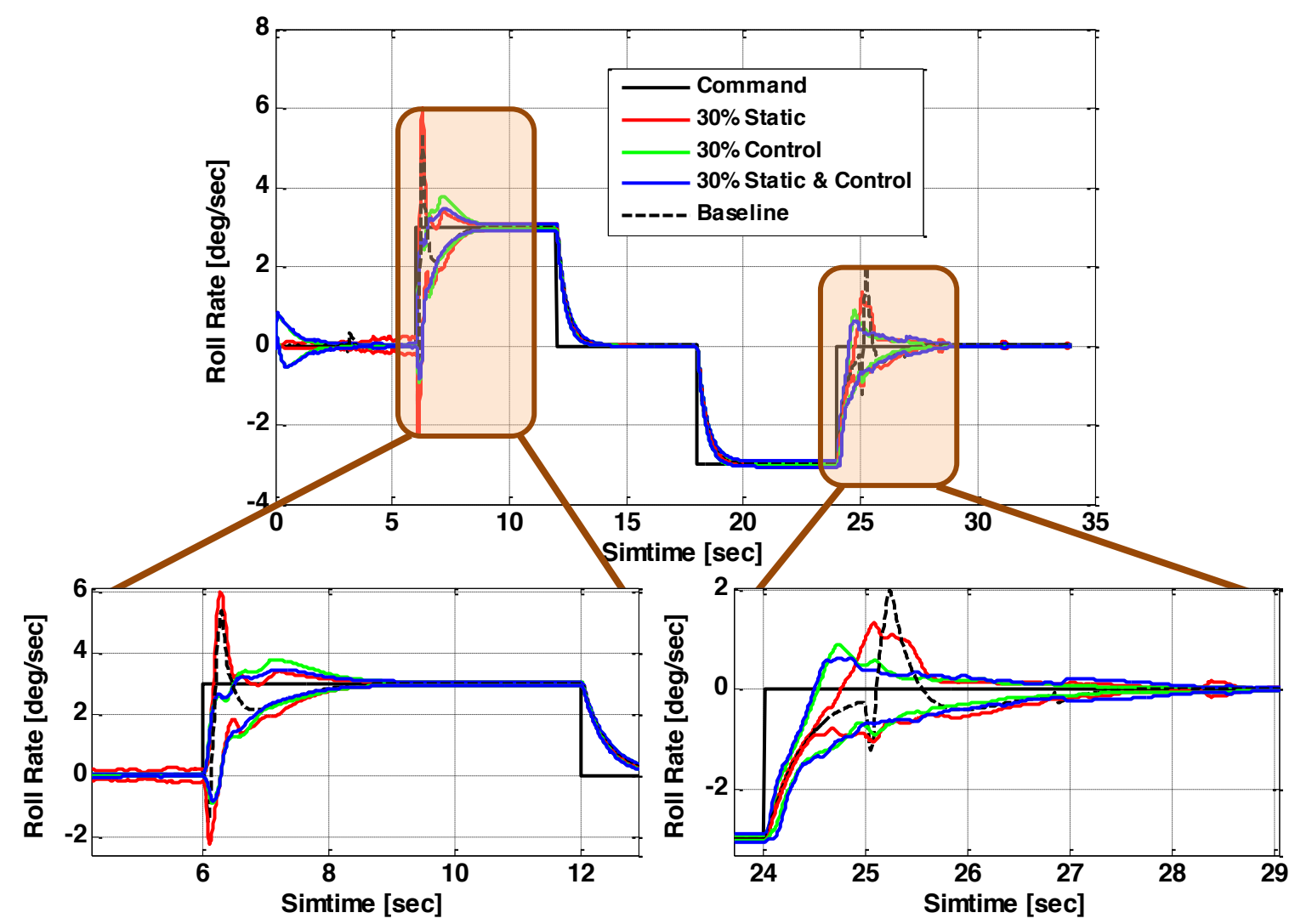

Figure 3-2: Standard deviation of roll rate responses for $30 \%$ plant uncertainty.

Table 3-1 shows the maximum steady state error for each uncertainty case. As mentioned in Section 1.10, maximum absolute steady state errors are computed between the standard deviation and the command. From the table, the control uncertainty case performs better than the static uncertainty case. However, it is hard to determine whether static uncertainty or control uncertainty degrades controller performance at this point. The static uncertainty case is closer to the baseline case, so if a better baseline flight condition is chosen, there is a possibility that the static uncertainty case may perform better if the baseline performance is better. The only conclusion drawn is that the static uncertainty case exhibits similar response to the baseline case, while the control uncertainty changes the vehicle response from the baseline. The case with static and control uncertainty exhibits similar performance to the control uncertainty case, indicating 
that any control uncertainty may change the controller performance from the baseline because the control effectiveness changes.

Table 3-1: Maximum steady state error for roll rate doublet with 30\% plant uncertainty.

\begin{tabular}{|l|c|}
\hline \multicolumn{1}{|c|}{ Aerodynamic Uncertainty } & $\begin{array}{c}\text { Max Absolute } \\
\text { Steady State Error } \\
(\mathbf{\%})\end{array}$ \\
\hline \hline $30 \%$ Static Uncertainty & 5.9 \\
\hline $30 \%$ Control Uncertainty & 3.8 \\
\hline $30 \%$ Static \& Control Uncertainty & 8.0 \\
\hline
\end{tabular}

Figure 3-3 depicts vehicle response to the same roll doublet command with $40 \%$ uncertainty modeling for static aerodynamics, control surface aerodynamics, and both for 350 simulation runs. Figure 3-4 compiles the standard deviation for each uncertainty case into one plot to compare controller performance between uncertainty cases.
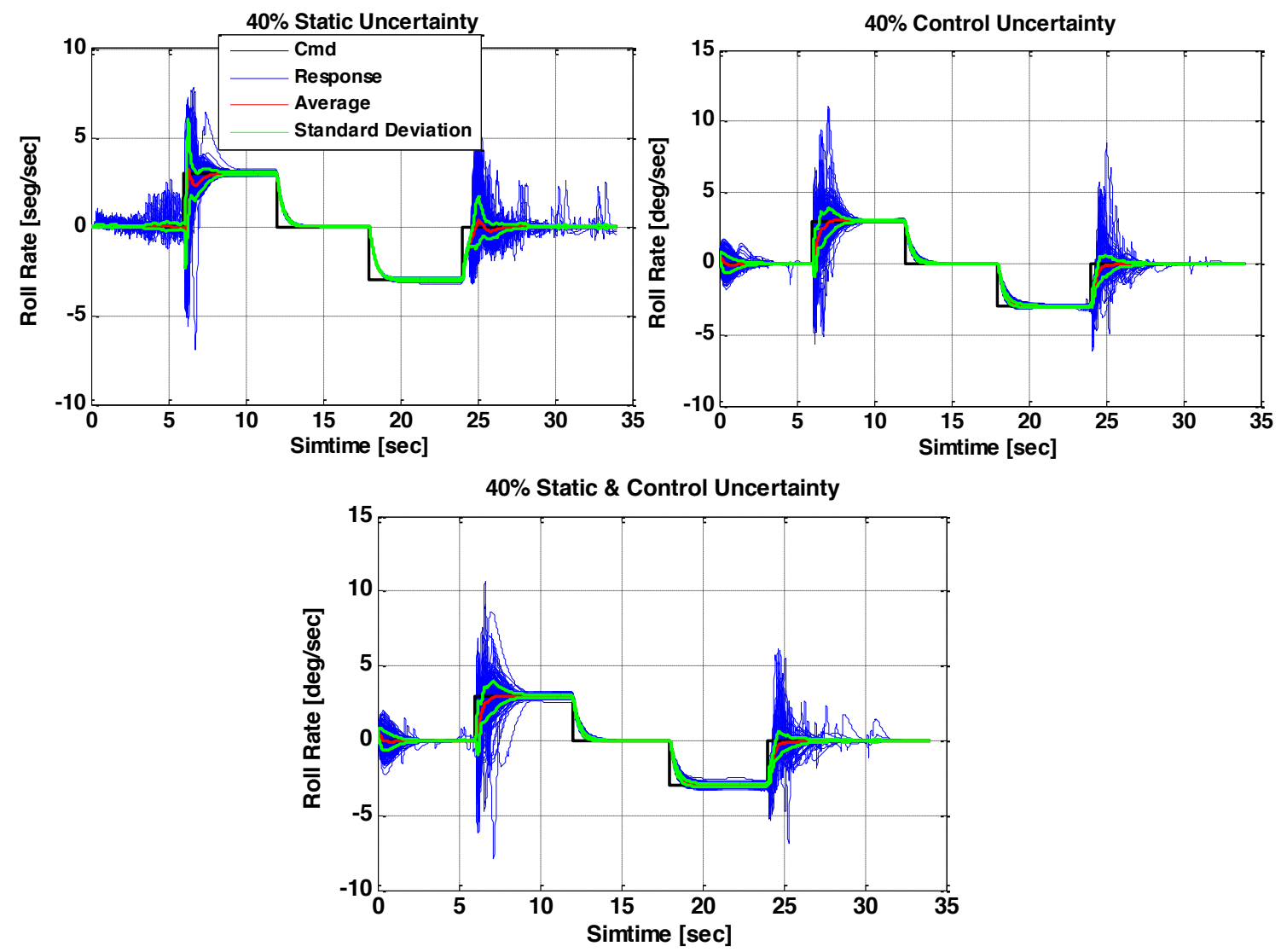

Figure 3-3: Roll rate response with $40 \%$ plant uncertainty. 


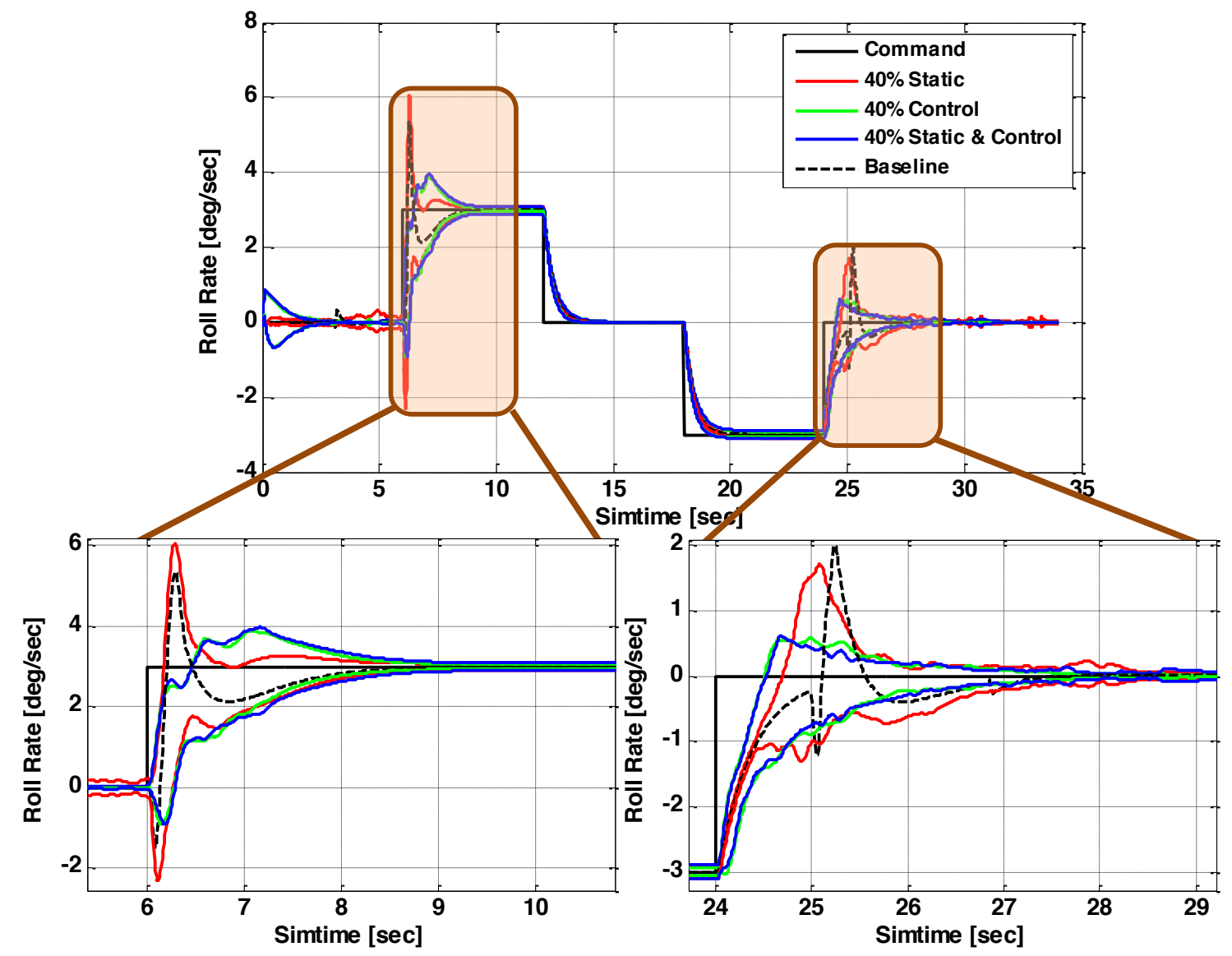

Figure 3-4: Standard deviations for roll rate response with $40 \%$ plant uncertainty.

The controller performance looks similar to the $30 \%$ uncertainty cases. Steady state error does not vary much from the $30 \%$ uncertainty case, as shown in Table 3-2 revealing that the roll axis is robust to $30 \%$ to $40 \%$ plant perturbations.

Table 3-2: Maximum steady state error for roll rate response with $40 \%$ plant uncertainty.

\begin{tabular}{|l|c|}
\hline \multicolumn{1}{|c|}{ Aerodynamic Uncertainty } & $\begin{array}{c}\text { Max Absolute } \\
\text { Steady State Error } \\
(\%)\end{array}$ \\
\hline \hline $40 \%$ Static Uncertainty & 7.4 \\
\hline $40 \%$ Control Uncertainty & 3.7 \\
\hline $40 \%$ Static \& Control Uncertainty & 5.1 \\
\hline
\end{tabular}


The aerodynamic uncertainties are further increased to $50 \%$ for each uncertainty case. The resulting controller performance for the 550 simulation run for each test case is shown in Figure 3-5.
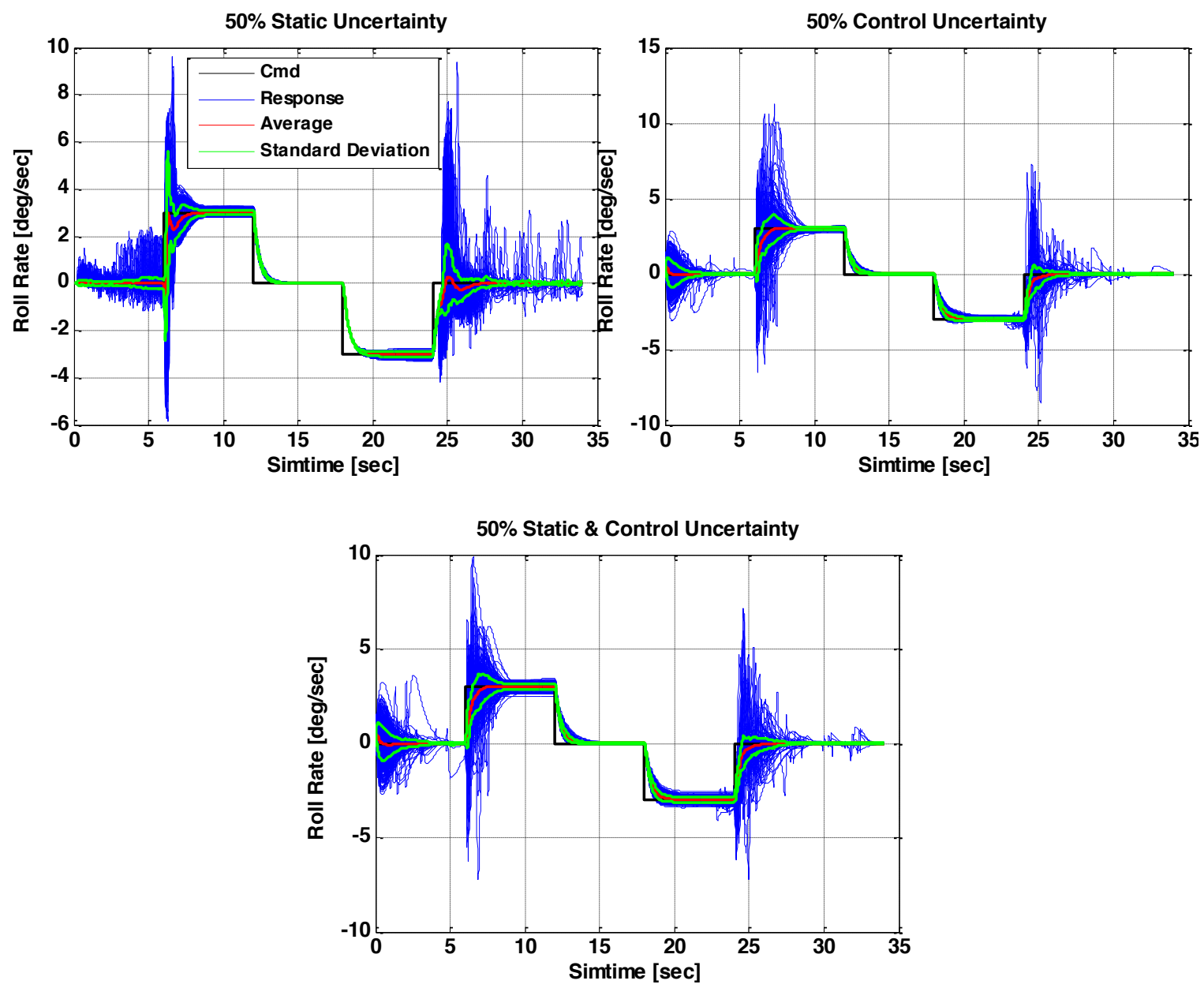

Figure 3-5: Roll rate response with 50\% plant uncertainty.

For the static uncertainty case, there are more oscillations caused from trimming error and more oscillations towards the end of the simulation caused by the axis saturation from the positive roll acceleration. Control surfaces are unable to cancel innate plant dynamics when the roll axis saturates. Roll axis saturation induces inherent axis coupling with the other two axes causing pitch rate and yaw rate to diverge from zero deg/sec. Coupling is caused from the aerodynamic nonlinearities of control effectors that are capable of applying moments to all three 
axes. Pitch rate and yaw rate excursion from zero requires more desired pitch rate and yaw rate acceleration in addition to an existing desired roll rate acceleration. The control allocator has to properly distribute control authority among three axes to achieve desired dynamics to close the error from the axis saturation. Axis saturation continually occurs if the control allocator is unable to solve for control deflections within actuator limits causing overshoots in all three axes until a proper set of control deflections are found. Therefore, control saturation degrades performance in other two axes causing roll oscillations seen from 25 to 35 seconds.

Figure 3-6 shows the standard deviation comparison for the three uncertainty cases.

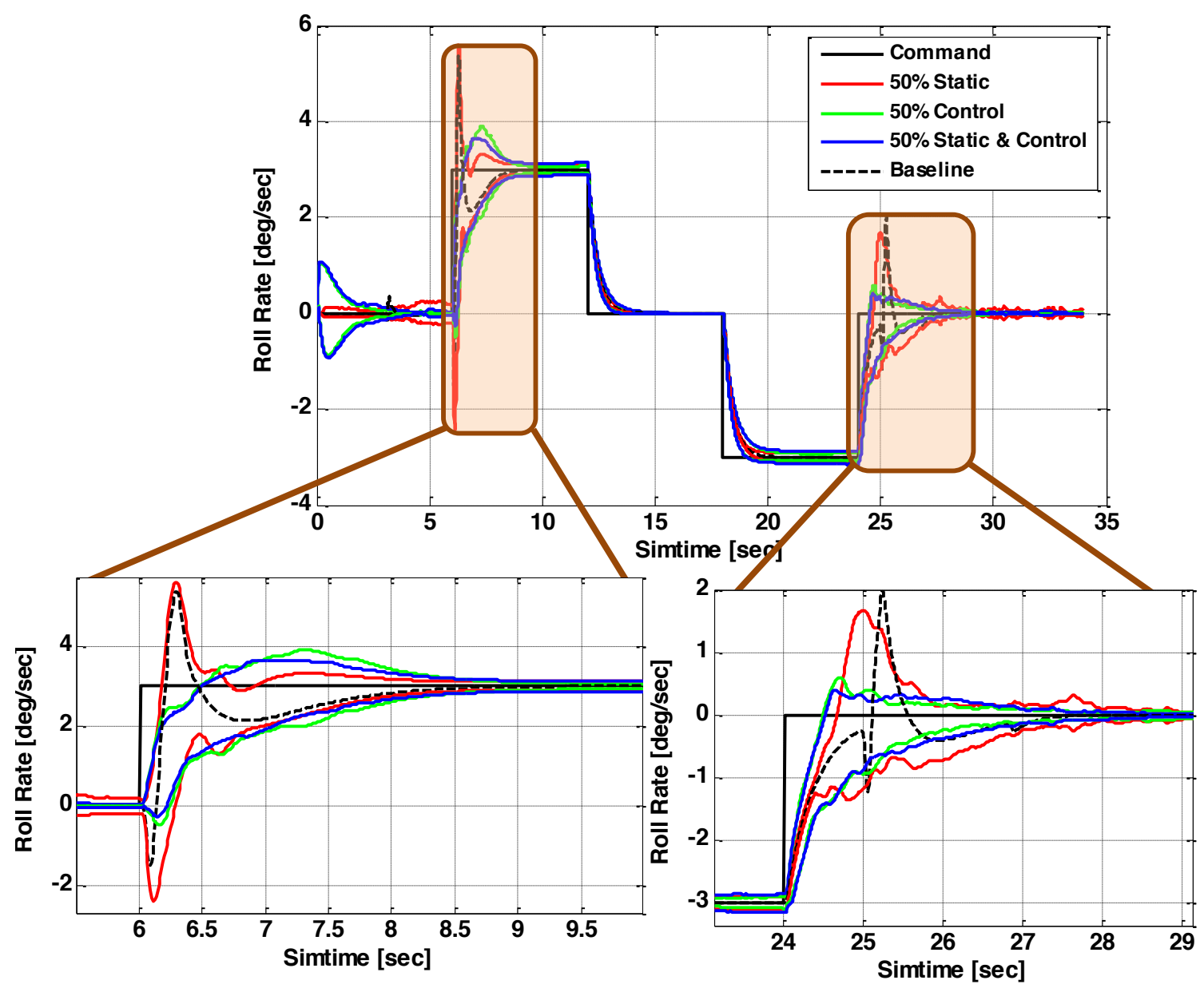

Figure 3-6: Standard deviation for roll rate responses with $\mathbf{5 0 \%}$ plant uncertainty. 
Number of simulation runs that display non-minimum phase zero behavior increases for the control uncertainty case, but not enough to cause the standard deviation to exhibit that behavior. The standard deviation for all three cases remains similar to the $30 \%$ and $40 \%$ case. Table $3-3$ tabulates the maximum absolute stead state error for each uncertainty case. Steady state errors remain within $3 \%$ and $8 \%$ error for the control uncertainty case and the static and control uncertainty case, which are similar values obtained from the $30 \%$ and $40 \%$ uncertainty case. The static uncertainty case shows an oscillation at around 27.5 seconds causing the absolute maximum error to go up to $11.2 \%$. However, roll rate tracking remains close to previous static uncertainty controller performances for the remainder of the simulation run for the $50 \%$ static uncertainty case.

Table 3-3: Standard deviation for roll rate response with $\mathbf{5 0 \%}$ plant uncertainty.

\begin{tabular}{|l|c|}
\hline \multicolumn{1}{|c|}{ Aerodynamic Uncertainty } & $\begin{array}{c}\text { Max Absolute } \\
\text { Steady State Error } \\
(\mathbf{\%})\end{array}$ \\
\hline \hline $50 \%$ Static Uncertainty & 11.2 \\
\hline $50 \%$ Control Uncertainty & 3.8 \\
\hline $50 \%$ Static \& Control Uncertainty & 5.4 \\
\hline
\end{tabular}

Figure 3-7 depicts the dynamic inversion controller performance after increasing plant uncertainty to $60 \%$ and running 600 simulations for each test case. 

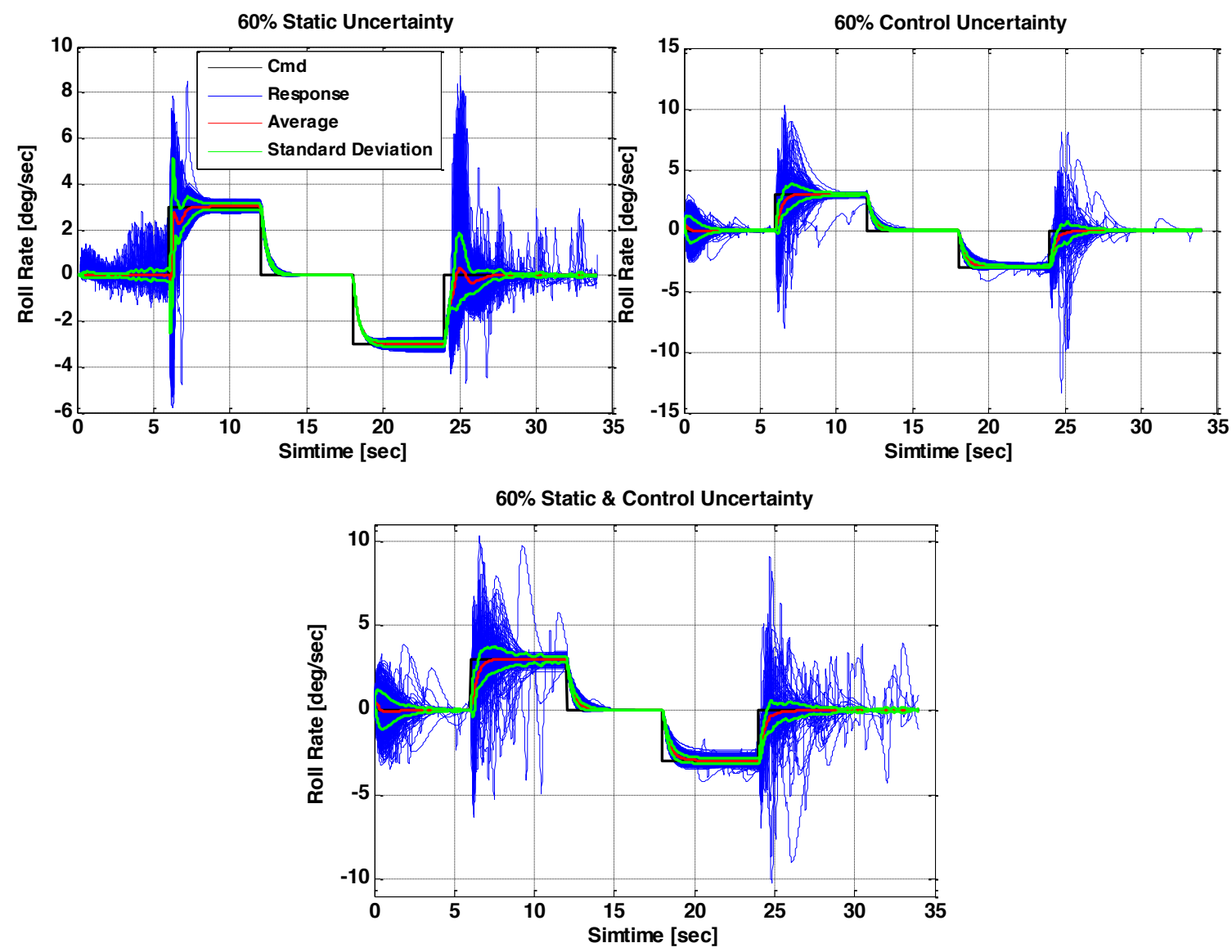

Figure 3-7: Roll rate response with $60 \%$ plant uncertainty.

Steady state errors are larger during positive roll accelerations, as well as negative roll accelerations, especially for the control uncertainty case and the static and control uncertainty case. The positive acceleration maneuvers for the control uncertainty case and the static and control uncertainty case are starting to induce more oscillations at higher magnitudes and drive the vehicle towards instability. Thus, demonstrating more static uncertainty is tolerable than control uncertainty. Table 3-4 shows maximum absolute steady state error response for the $60 \%$ uncertainty case. 
Table 3-4: Maximum steady state error for roll rate response with $60 \%$ plant uncertainty.

\begin{tabular}{|l|c|}
\hline \multicolumn{1}{|c|}{ Aerodynamic Uncertainty } & $\begin{array}{c}\text { Max Absolute } \\
\text { Steady State Error } \\
(\mathbf{\%})\end{array}$ \\
\hline \hline $60 \%$ Static Uncertainty & 8.9 \\
\hline $60 \%$ Control Uncertainty & 7.8 \\
\hline $60 \%$ Static \& Control Uncertainty & 14.6 \\
\hline
\end{tabular}

The standard deviation for the control uncertainty case does not capture some simulation runs that cause the vehicle to stop tracking roll rate well, seen in Figure 3-7, despite the steady state error for the control uncertainty case being less than the static uncertainty. The dynamic inversion controller is able to tolerate $60 \%$ static aerodynamic uncertainty while tracking roll rate commands within allowable steady state error limits, indicating the controller is more robust to static uncertainty than control uncertainty. The dynamic inversion controller is robust to up to $50 \%$ control uncertainty before the vehicle starts to show signs of instability. The control uncertainty is the limiting factor for controller performance in the presence of static or control uncertainty. The vehicle is unable to track the roll rate response within an acceptable tolerance when $60 \%$ static and control uncertainties were introduced since the controller starts to drive the vehicle towards instability for $60 \%$ uncertainty in control surface aerodynamics. For all three uncertainty cases, the controller is able to track roll rate commands well as long as there are no axis saturation induced by producing a positive roll and pitch acceleration simultaneously.

Pitch rate doublet tracking is tested for the same set of plant uncertainties in order to evaluate dynamic inversion controller robustness in the pitch axis. Figure 3-8 depicts controller performances for 300 simulation runs for $30 \%$ plant uncertainties. 

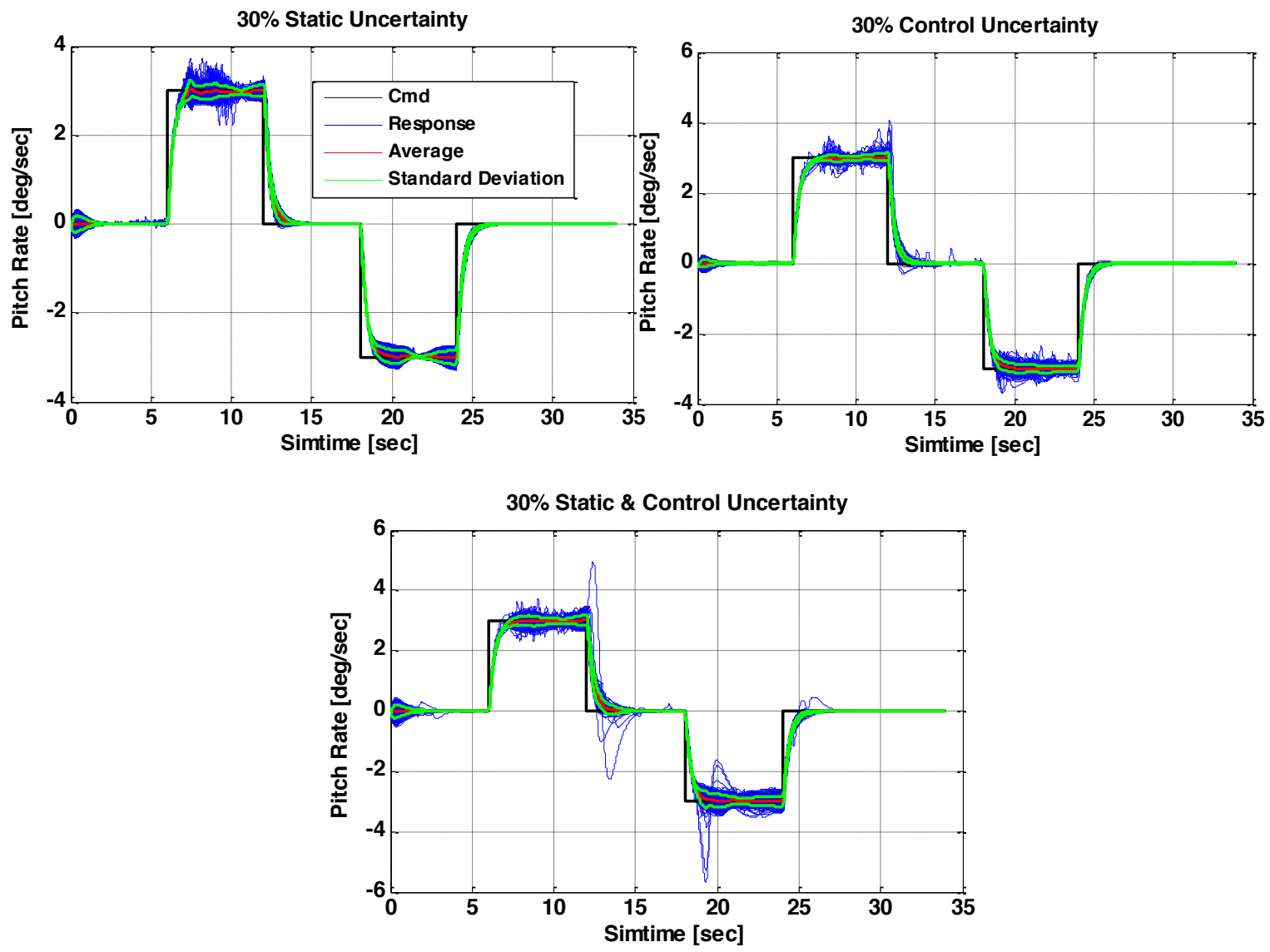

Figure 3-8: Pitch rate response with $30 \%$ plant uncertainty.

All uncertainty cases show oscillations about a steady state value between 7 and 12 seconds, and again between 19 and 24 seconds, which was not observed in the baseline controller performance. The error between the plant and controller model prevents exact plant dynamic cancellation using control effectors resulting in axes coupling. A non-zero pitch rate causes $\alpha$ to increase or decrease depending on the sign of the pitch rate command. $\alpha$ affects the coefficient computation for the controller because all aerodynamic coefficients are functions of $\alpha$, Mach, and $\beta$. The inherent pitch, roll, and yaw acceleration from the wing-body changes as $\alpha$ changes, which consequently affects the desired acceleration computation. If there are static aerodynamic modeling errors between the plant and the on-board controller model, the desired acceleration computation may overestimate or underestimate the amount of plant acceleration to compensate with control 
deflections. Even if the control effectiveness between the plant and the controller is the same, the dynamic inversion solves for control deflections achieving inaccurate desired accelerations, resulting in overshoots in pitch, roll, and yaw. For the control uncertainty case, the oscillations are larger in magnitude because the control allocator uses a control effectiveness matrix containing errors. The dynamic inversion controller computes the local control derivative containing aerodynamic terms with error, causing the vehicle to overshoot or undershoot more depending on the error of the effectiveness matrix compared to the plant model. The pitch axis seems more sensitive to uncertainty terms compared to the roll axis since the RLV aerodynamic model is a function of $\alpha$. Roll maneuvers only affect $\mu$, and may change $\alpha$ slightly if axis saturation causes the vehicle to produce a pitch rate. RLV's aerodynamic model is not a function of $\mu$ so uncertainties do not seem to affect controller tracking when a non-zero roll rate is commanded.

Figure 3-9 depicts the standard deviation for each $30 \%$ uncertainty case for the pitch doublet maneuver. Table 3-5 shows the result of maximum steady state error computed from the standard deviation.

Table 3-5: Maximum steady state error for pitch rate response with $30 \%$ plant uncertainty.

\begin{tabular}{|l|c|}
\hline \multicolumn{1}{|c|}{ Aerodynamic Uncertainty } & $\begin{array}{c}\text { Max Absolute } \\
\text { Steady State Error } \\
\mathbf{( \% )}\end{array}$ \\
\hline \hline $30 \%$ Static Uncertainty & 5.5 \\
\hline $30 \%$ Control Uncertainty & 5.4 \\
\hline $30 \%$ Static \& Control Uncertainty & 6.5 \\
\hline
\end{tabular}




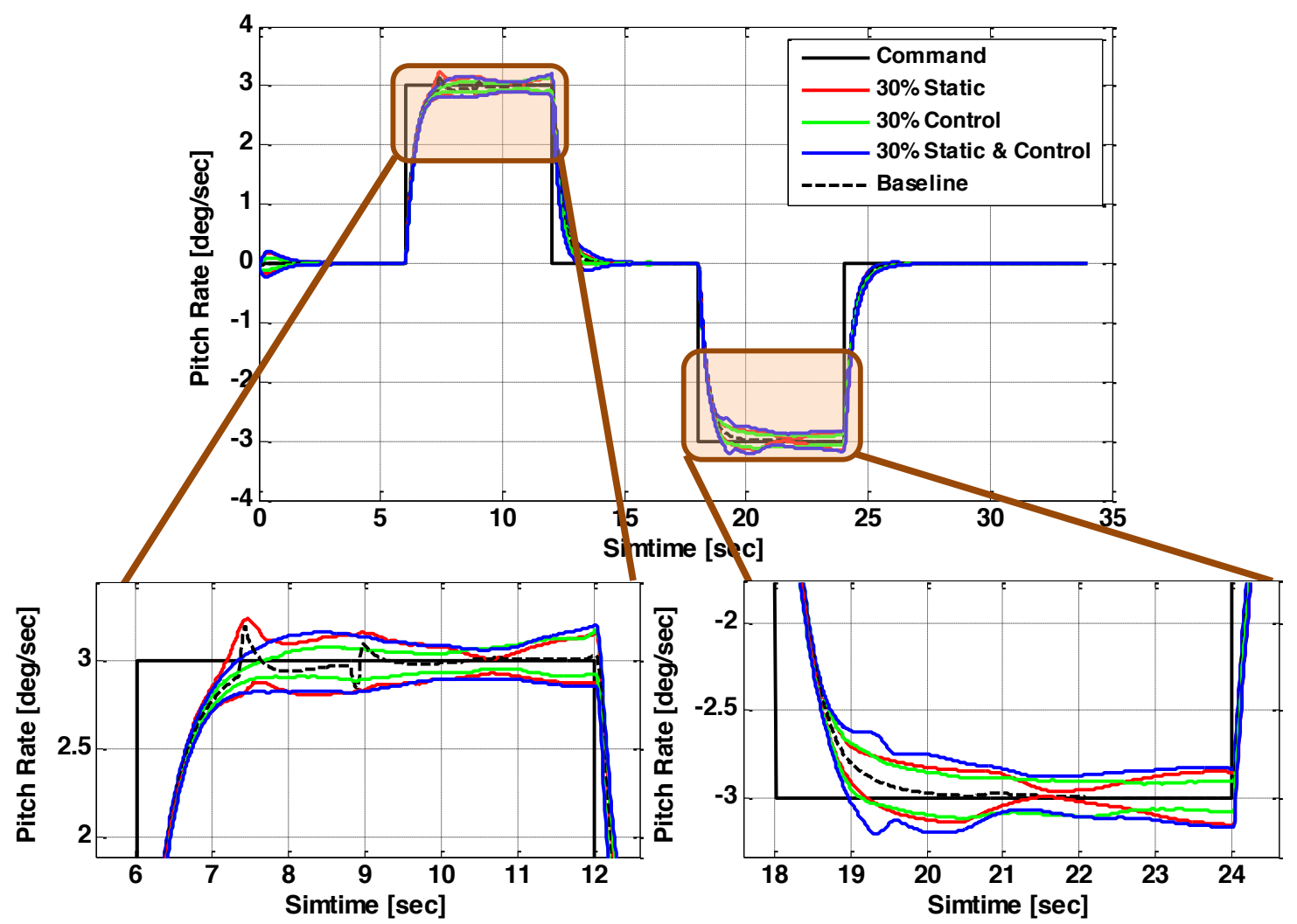

Figure 3-9: Standard deviation for pitch rate response with $30 \%$ plant uncertainty.

The dynamic inversion controller performs well according to the figures of merit. Like the roll rate doublet cases, the static uncertainty case follows the baseline results more than the control uncertainty case. Static aerodynamic error between the plant and the controller does not alter the control effectiveness between the plant and the controller so a spike in the pitch rate response is seen at around 7 and 9 seconds, similar to the baseline. The local control slope can drastically change over a break point, which causes the dynamic inversion to solve for inadequate control deflections to achieve a desired moment resulting in the spikes in pitch rate. The control uncertainty case and the static and control uncertainty case both exhibit different tracking behavior from the baseline. Errors in the control effectiveness matrix cause the vehicle to produce body accelerations that causes the vehicle to fly at a different flight condition. 
Figure 3-10 displays the simulation results from 350 runs for each uncertainty case when the plant perturbations are increased to $40 \%$.
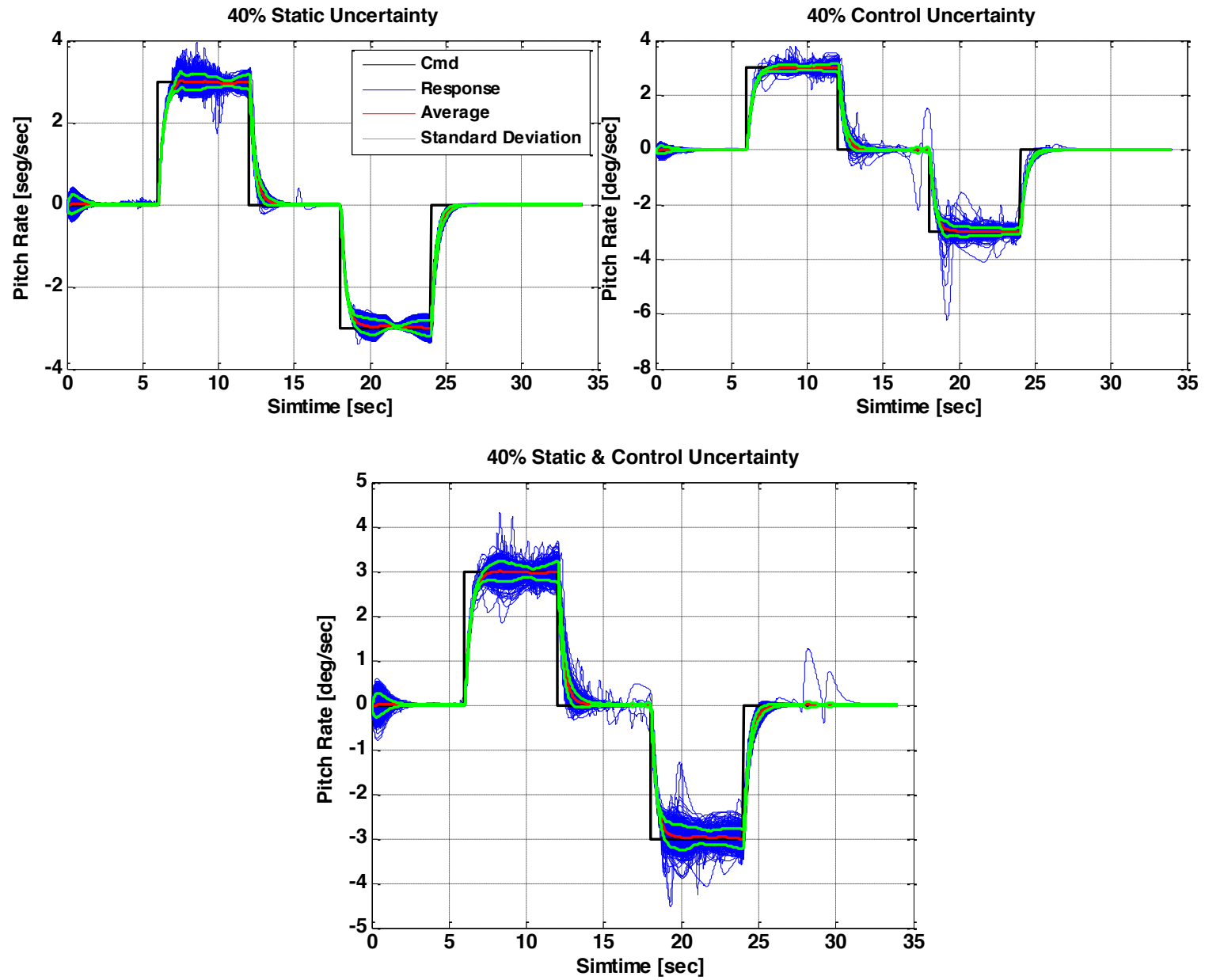

Figure 3-10: Pitch rate response with $40 \%$ plant uncertainty.

Unlike the roll doublet case, the pitch axis is more sensitive to changes in uncertainty magnitudes. The increase in error between the plant model and the on-board controller causes larger oscillations. The static uncertainty case still shows nice steady state response when zero $\mathrm{deg} / \mathrm{sec}$ pitch rate is commanded. However, the control uncertainty case and the static and control uncertainty case are starting show more oscillations during the same zero deg/sec command. One of the control uncertainty simulation runs demonstrates the vehicle is nearing instability during a $-3 \mathrm{deg} / \mathrm{sec}$ pitch rate maneuver. 
The standard deviation for each uncertainty case from Figure 3-10 is plotted in Figure 3-11. The maximum steady state error between the standard deviation and the pitch rate command are shown in Table 3-6. Although, the standard deviation shows acceptable controller performance, the dynamic inversion controller may be risky to use for $40 \%$ uncertainties in control surface aerodynamics. The standard deviation doesn't capture some simulation runs from Figure 3-10 that depicts the control uncertainty case containing undesirable oscillations caused by the vehicle approaching instability.

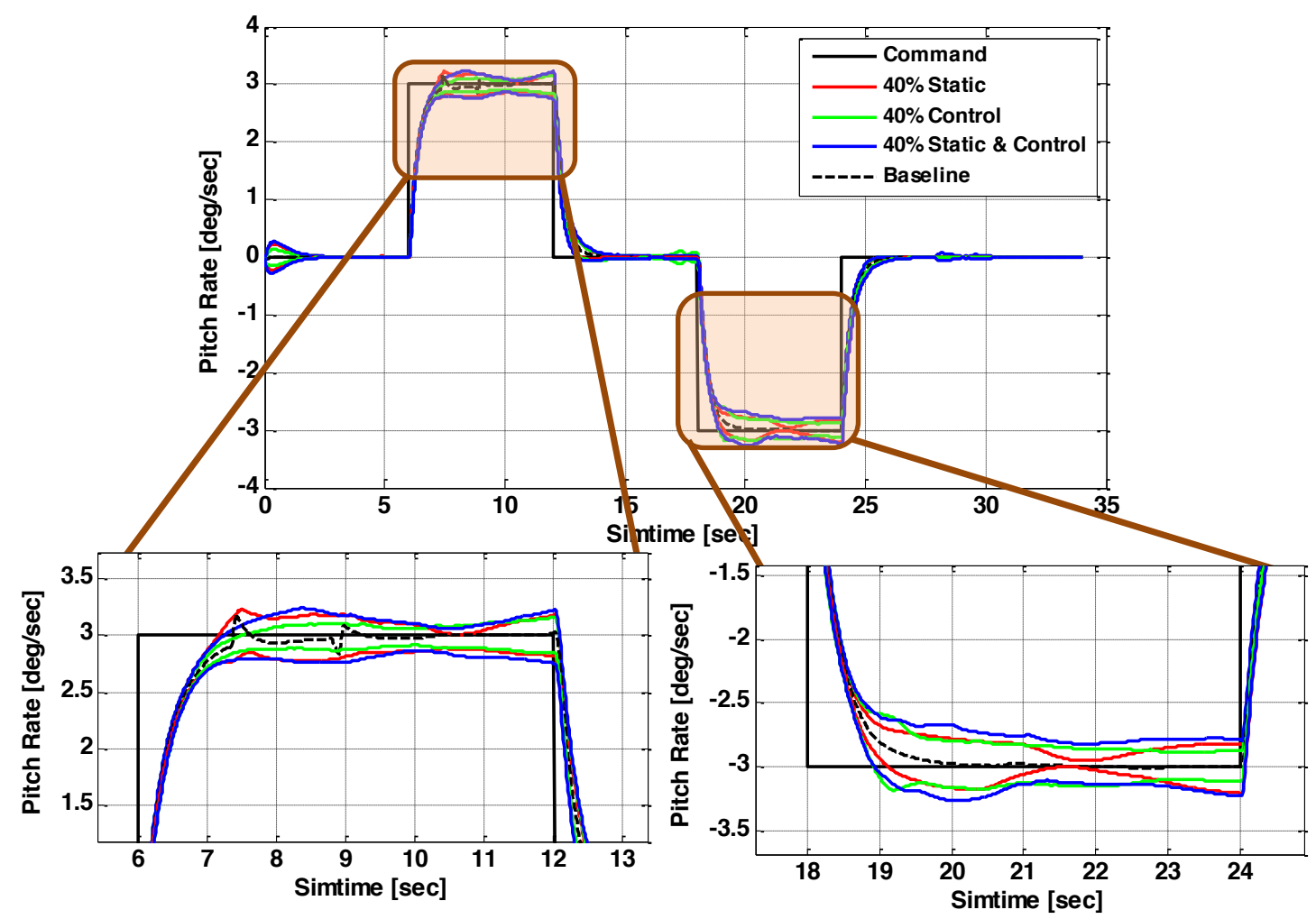

Figure 3-11: Standard deviation for pitch rate response with $40 \%$ plant uncertainty. 
Table 3-6: Maximum steady state error for pitch rate response with $40 \%$ plant uncertainty.

\begin{tabular}{|l|c|}
\hline \multicolumn{1}{|c|}{ Aerodynamic Uncertainty } & $\begin{array}{c}\text { Max Absolute } \\
\text { Steady State Error } \\
(\%)\end{array}$ \\
\hline \hline $40 \%$ Static Uncertainty & 6.9 \\
\hline $40 \%$ Control Uncertainty & 5.8 \\
\hline $40 \%$ Static \& Control Uncertainty & 8.7 \\
\hline
\end{tabular}

Same pitch rate doublets are input into the dynamic inversion controller after aerodynamic modeling uncertainty is increased to $50 \%$. The control uncertainty case has three simulation runs that went unstable after the first negative pitch acceleration command from 3 $\mathrm{deg} / \mathrm{sec}$ pitch rate to zero deg/sec. The static and control uncertainty case has two simulation runs where the vehicle lost control. If the number of simulation runs is increased, by the Law of Large Numbers, the occurrence of instability will equate for both cases. However, simulation runs are kept at 550 runs for $50 \%$ uncertainty cases due to the scope of this thesis. Simulation cases where the vehicle went unstable are considered "outliers" when plotting the vehicle responses to pitch rate doublet commands. In reality, they are not considered "outliers;" but in order to capture the majority of the controller performance in a standard deviation calculation the "outliers" are removed so large standard deviations were not computed from unstable vehicle performance. Figure 3-12 depicts all 550 simulation runs for each uncertainty case without "outliers." 

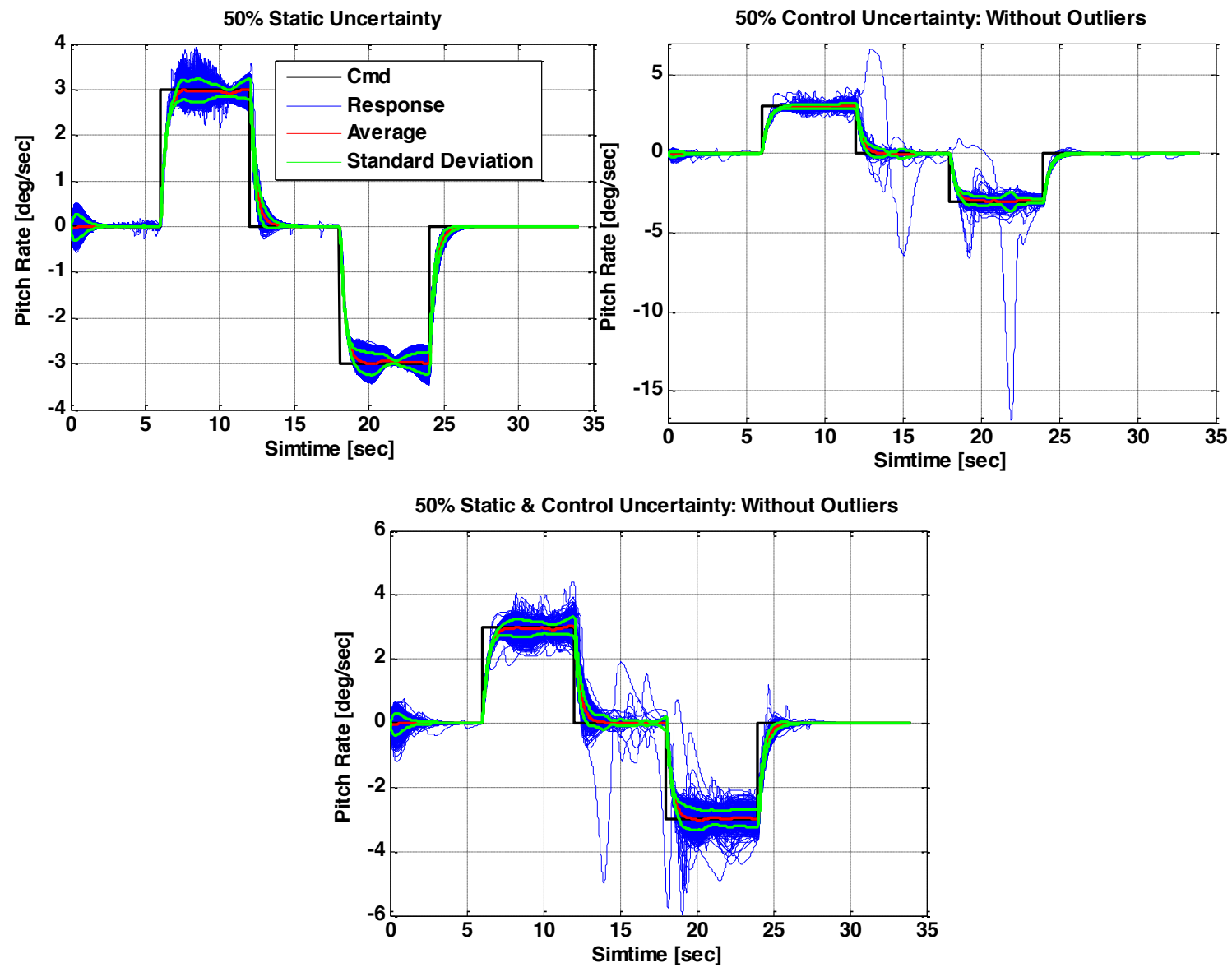

Figure 3-12: Pitch rate responses with 50\% plant uncertainty.

Without "outliers," there are still more simulation runs that exhibit the vehicle getting close to instability when performing a negative pitch maneuver for the control uncertainty case and the static and control uncertainty case. Simulation runs from static uncertainty do not show indications of the vehicle nearing instability. The static uncertainty cases has larger oscillations about steady state values for non-zero pitch rate commands, but is capable of holding a zero deg/sec command with minimal error. The integral term is able to close the error on pitch rate command when the flight condition does not change with $\alpha$.

Figure 3-13 plots the standard deviation for each set of uncertainty cases. Table 3-7 shows maximum absolute steady state error between the standard deviation and the command without including "outliers." Due to the "outliers," the control uncertainty case and the static and 
control uncertainty case exhibit unacceptable controller performance even if the steady state error says different. The static uncertainty case shows that the controller tracking is acceptable even with $50 \%$ static aerodynamic error.

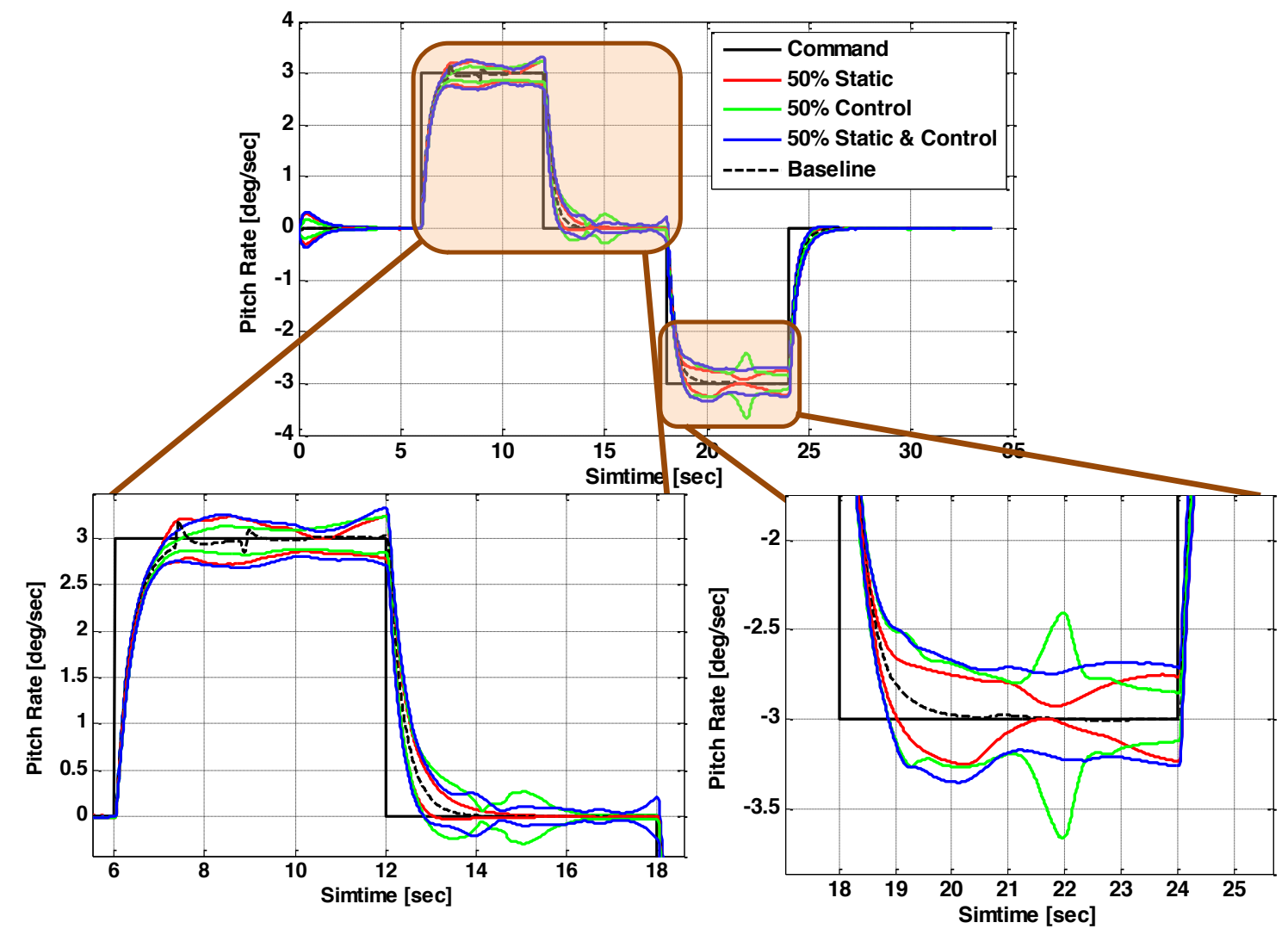

Figure 3-13: Standard deviation for pitch rate response with 50\% plant uncertainty.

Table 3-7: Maximum steady state error for pitch rate response with 50\% plant uncertainty.

\begin{tabular}{|l|c|}
\hline \multicolumn{1}{|c|}{ Aerodynamic Uncertainty } & $\begin{array}{c}\text { Max Absolute } \\
\text { Steady State Error } \\
(\mathbf{\%})\end{array}$ \\
\hline \hline $50 \%$ Static Uncertainty & 8.7 \\
\hline $50 \%$ Control Uncertainty & 22.2 \\
\hline $50 \%$ Static \& Control Uncertainty & 10.4 \\
\hline
\end{tabular}


Figure 3-14 depicts 600 simulations runs for each pitch rate doublet case when aerodynamic uncertainty increases to $60 \%$. The control uncertainty case does not have any simulation runs that cause the vehicle to lose control, but the static and control uncertainty case contains 5 simulation runs that are considered "outliers." As previously mentioned, the 600 simulations runs may not have been enough to encompass uncertainty combinations that result in the vehicle going unstable for the control uncertainty case. However, one simulation run demonstrates a case where the controller loses tracking between 12 and 24 seconds during a negative pitch rate maneuver before regaining control, indicating a $60 \%$ control surface aerodynamic modeling error is unacceptable.
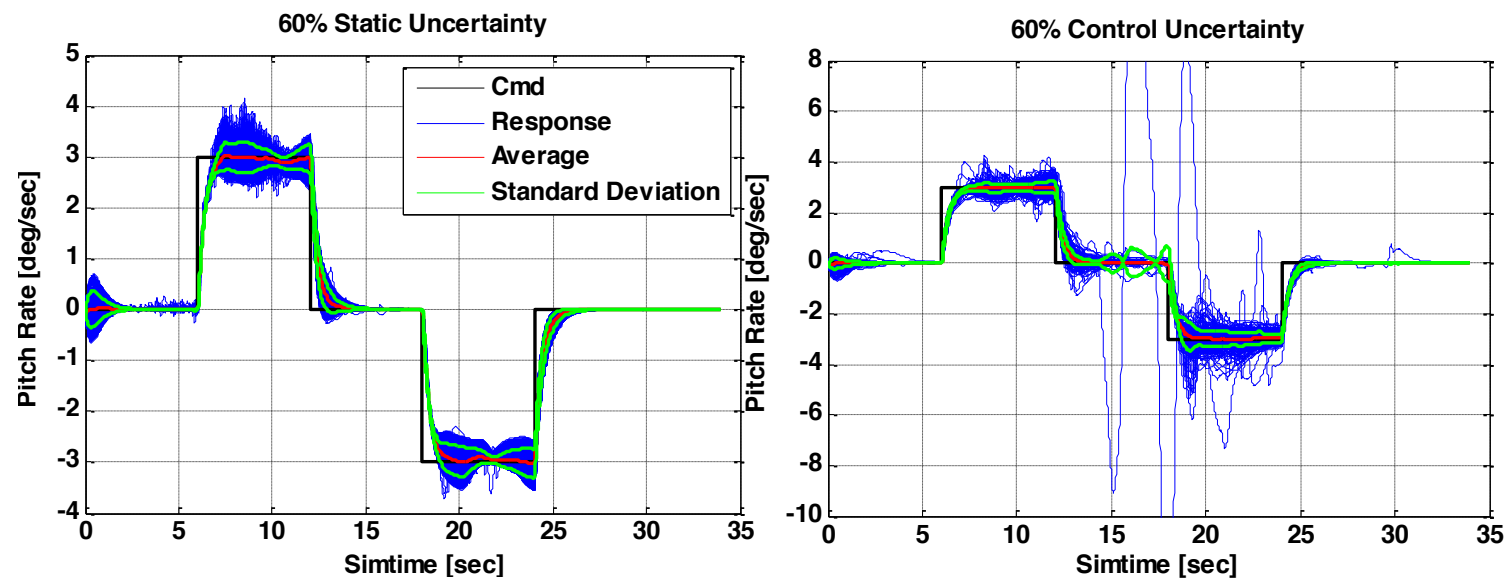

$60 \%$ Static \& Control Uncertainty: Without Outliers

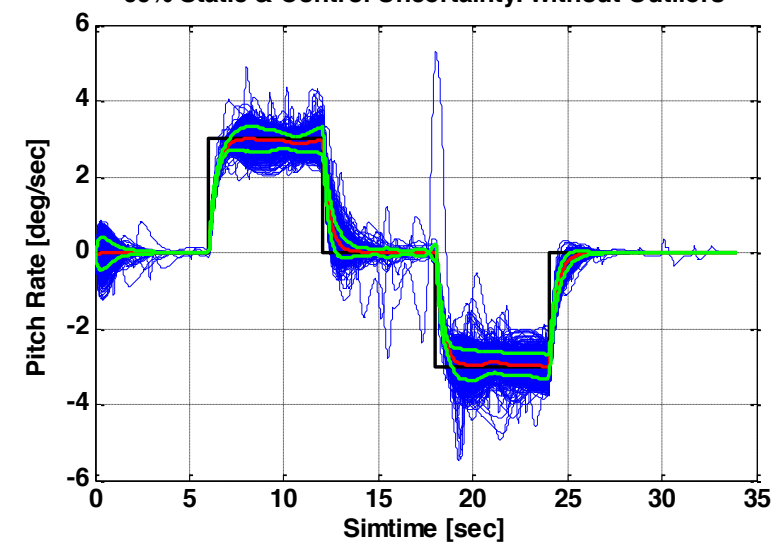

Figure 3-14: Pitch rate response with $60 \%$ plant uncertainty. 
Figure 3-15 depicts the standard deviation for the three uncertainty cases, and Table 3-8 provides the maximum steady state error.

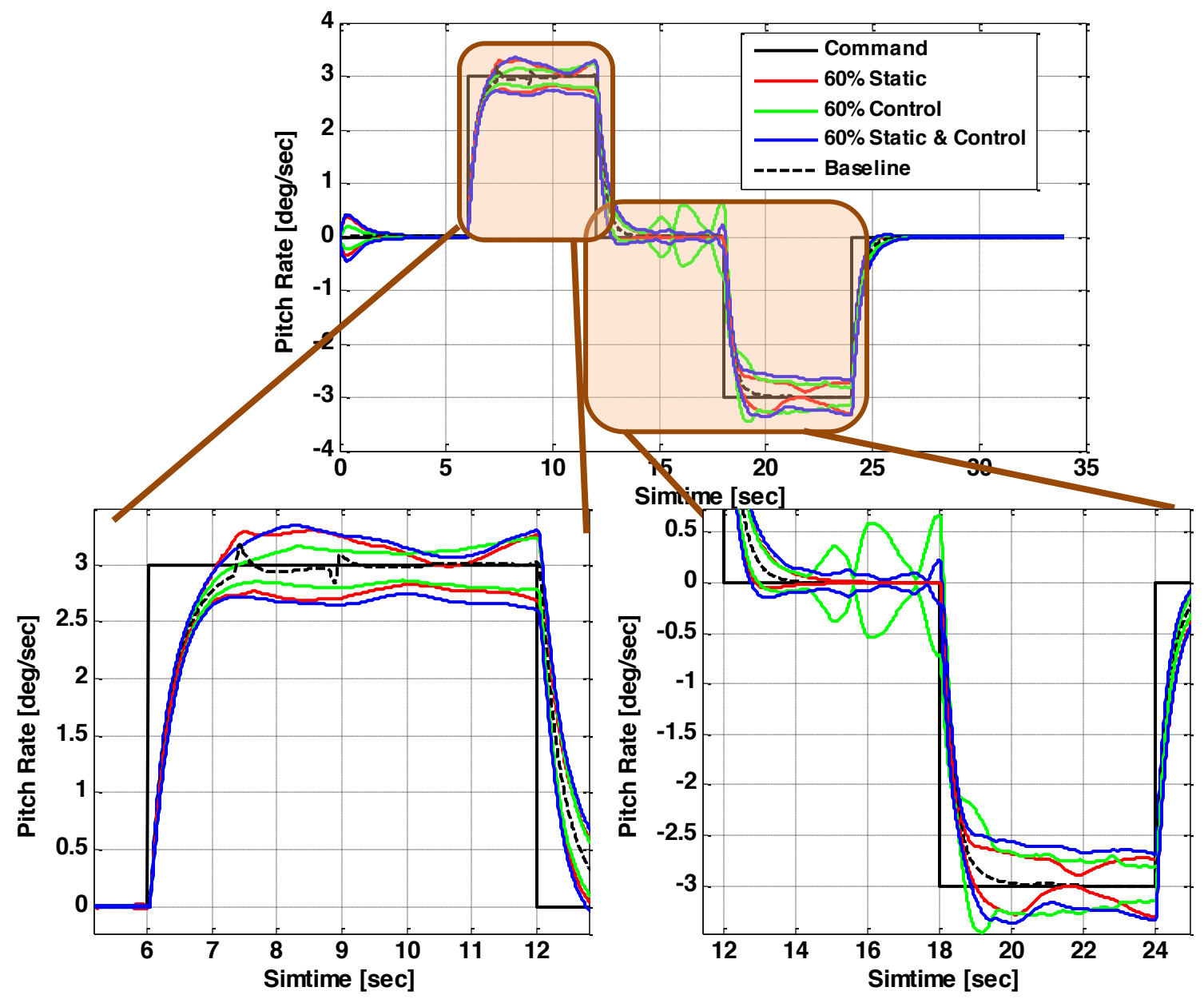

Figure 3-15: Standard deviation for pitch rate response with $60 \%$ plant uncertainty.

Table 3-8:Maximum steady state error for pitch rate response with $60 \%$ plant uncertainty.

\begin{tabular}{|l|c|}
\hline \multicolumn{1}{|c|}{ Aerodynamic Uncertainty } & $\begin{array}{c}\text { Max Absolute } \\
\text { Steady State Error } \\
(\mathbf{\%})\end{array}$ \\
\hline \hline $60 \%$ Static Uncertainty & 10.6 \\
\hline $60 \%$ Control Uncertainty & 24.1 \\
\hline $60 \%$ Static \& Control Uncertainty & 13.2 \\
\hline
\end{tabular}


The dynamic inversion controller tracking is unacceptable for $60 \%$ aerodynamic uncertainty according to the figures of merit. The pitch axis is more sensitive to perturbations in the plant compared to the roll axis. $60 \%$ static uncertainty was tolerated for the roll axis, but unacceptable for the pitch axis. Control uncertainty limits the amount of plant perturbations the controller can tolerate in the pitch axis, much like the roll axis. Control uncertainties induce more frequent and higher magnitude oscillations that drive the vehicle unstable. The dynamic inversion controller can track pitch rate commands within acceptable steady state error limits with $40 \%$ of aerodynamic modeling error between the on-board controller model and the plant if control uncertainty exists.

The same set of uncertainty cases are used to evaluate the dynamic inversion robustness in the yaw axis. Figure 3-16 shows the yaw rate response to a doublet command with $30 \%$ aerodynamic uncertainty for 300 simulation runs. The baseline controller results shows the control effectiveness matrix is close to singular when $\beta$ is equal to zero. A pseudo inverse solution of a singular matrix approaches infinity causing the control allocator to command large control deflections exceeding actuator limits when positive yaw acceleration is commanded. This same behavior is exhibited by all three uncertainty cases when the first positive yaw step is commanded at 6 seconds. Yaw axis saturation degrades pitch and roll rate performance since moments are coupled from control surface aerodynamics. 

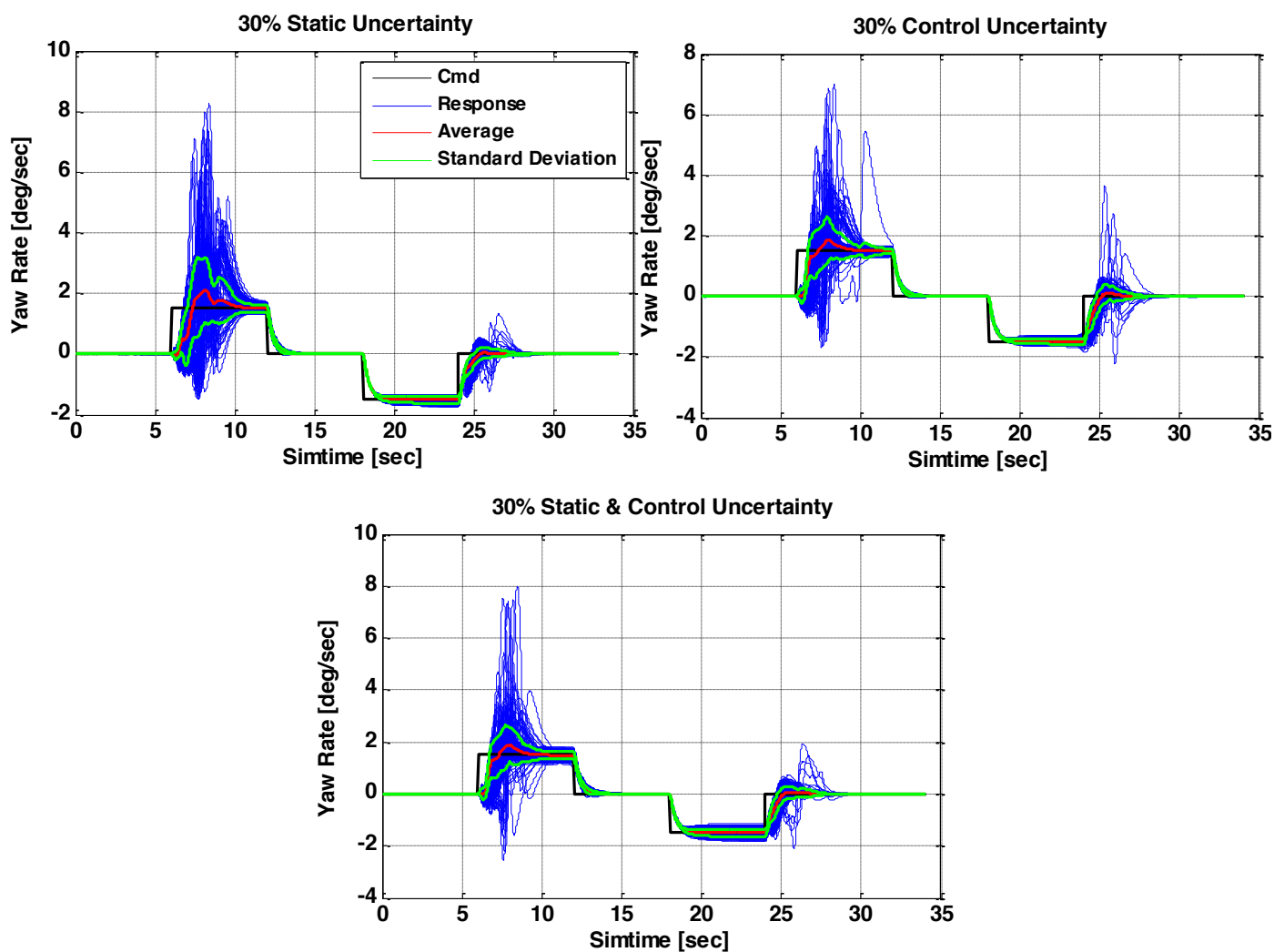

Figure 3-16: Yaw rate response with 30\% plant uncertainty

Uncertainty errors cause pitch and roll rate to diverge from zero deg/sec resulting in yaw rate oscillation at the second positive yaw step input at 24 seconds. Static aerodynamic errors cause the RLV to achieve wrong desired dynamics, even if the control allocator finds a control solution within actuator limits, since inherent plant dynamics are calculated with error. Control surface aerodynamic error will prevent the vehicle from achieving desired dynamics because the control deflection solution from the actuators are being computed using a control effectiveness matrix containing errors. Both static and control aerodynamic errors prevents inherent plant dynamics cancellation causing pitch rate and roll rate excursion from zero deg/sec even if control saturation does not occur. Tracking error prompts the prefilter to command a pitch and roll acceleration in addition to a desired yaw acceleration to perform the step maneuver. Body rate responses overshoot until enough control power exists to achieve desired body rate accelerations. 
Figure 3-17 displays the standard deviations for three uncertainty cases from the yaw rate response from Figure 3-16. Table 3-9 shows corresponding maximum steady state error between the standard deviation and the doublet command.

Table 3-9: Maximum steady state error for yaw rate response with $30 \%$ plant uncertainty.

\begin{tabular}{|l|c|}
\hline \multicolumn{1}{|c|}{ Aerodynamic Uncertainty } & $\begin{array}{c}\text { Max Absolute } \\
\text { Steady State Error } \\
(\mathbf{\% )}\end{array}$ \\
\hline \hline $30 \%$ Static Uncertainty & 68.2 \\
\hline $30 \%$ Control Uncertainty & 31.8 \\
\hline $30 \%$ Static \& Control Uncertainty & 26.5 \\
\hline
\end{tabular}

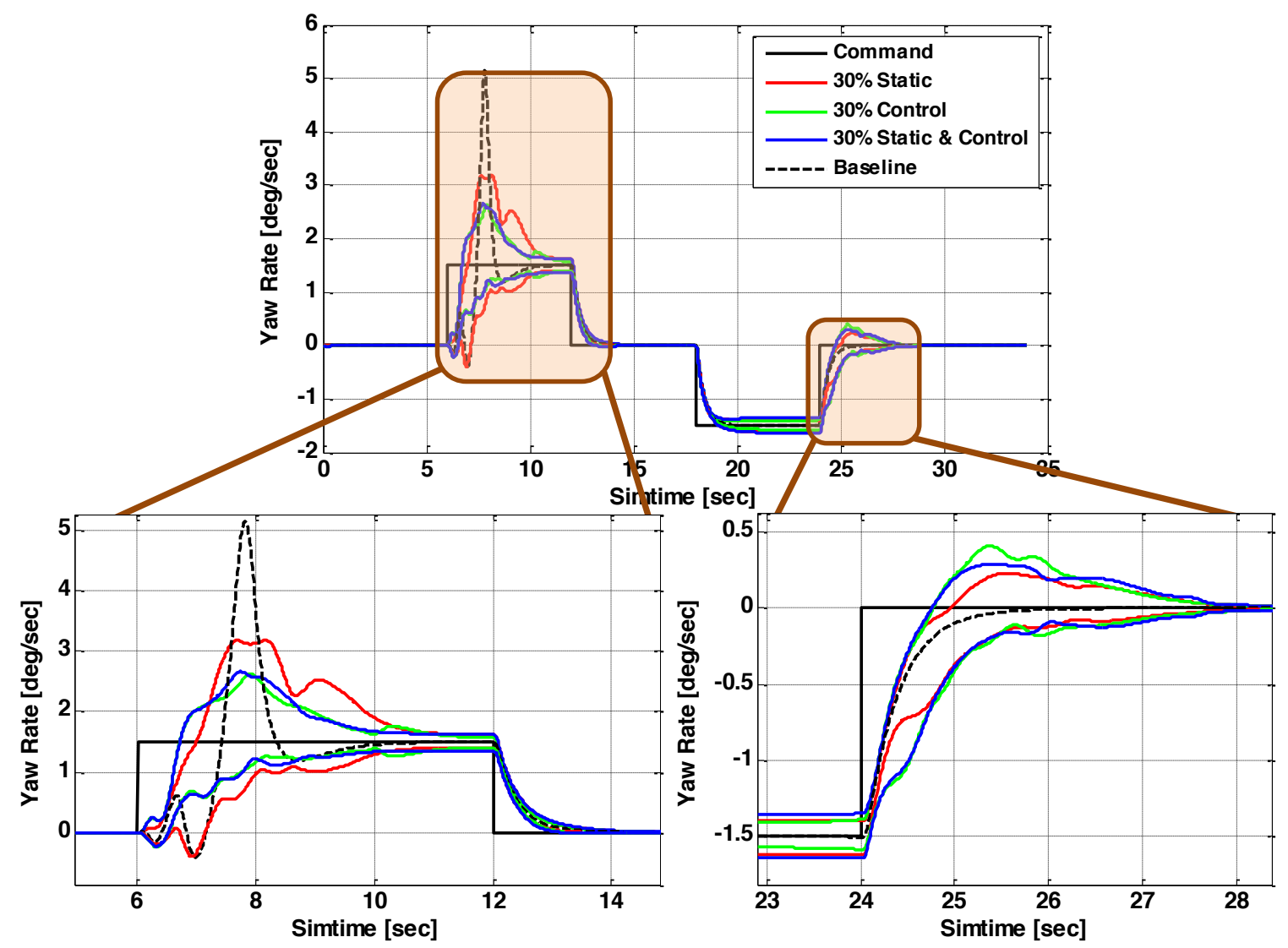

Figure 3-17: Standard deviation for yaw rate response with $30 \%$ plant uncertainty.

Aerodynamic uncertainties prevent the static uncertainty case and the control uncertainty case from closing the yaw rate error caused by control saturations as quickly as the baseline case. 
The overshoot caused by the initial yaw axis saturation produces maximum absolute steady state errors beyond acceptable limits as seen in Table 3-9. Aside from the initial yaw rate overshoot at the first positive step input, the dynamic inversion controller is able to track the yaw doublet well, especially negative yaw rate step inputs.

The control allocator uses a pseudo inverse solution to determine control deflection commands, which does not take actuator rate limits or actuator position limits into account so control saturation may occur even if control power may exist. Better methods are available to solve underdetermined system that poses the control allocation problem into an optimal formulation including actuator limits, like a linear programmer. Using a linear programmer may improve the tracking performance of the dynamic inversion controller for the initial step input if a control allocator can solve for an optimal solution within actuator limits.

Side slip varies when yaw rate is non-zero. Therefore, inherent pitch, roll, and yaw acceleration due to the bare airframe changes as $\beta$ changes. Varying inherent plant dynamics are difficult for the integrator to close the error on yaw rate command in the presence of uncertainty errors. This is the reason for steady state error between 20 and 24 seconds for a negative yaw rate maneuver even if the steady state error is close to zero for the first negative yaw rate maneuver between and 18 seconds. Aerodynamic error between the plant and the controller model prevents innate plant dynamic cancellation causing oscillations in other axes like roll and pitch. An overshoot in roll or pitch often induces an overshoot in yaw caused by moment coupling with control surface deflections.

Much like the roll doublet tests and pitch doublet tests, the static uncertainty case mimics the baseline tracking better than the control uncertainty case. Static uncertainty does not generate as much error between the plant and the on-board controller model to make the vehicle diverge 
from the baseline performance as much as the control uncertainty. The control uncertainty changes the control effectiveness of the plant causing the vehicle to achieve a different flight condition, so the standard deviation for the control uncertainty case and the static and control uncertainty case tracks the doublet command differently from the baseline without as much overshoot at the initial positive step input.

Increasing aerodynamic uncertainty to $40 \%$ degrades the yaw rate doublet tracking further. Figure 3-18 shows the 350 simulation run for the three uncertainty cases.
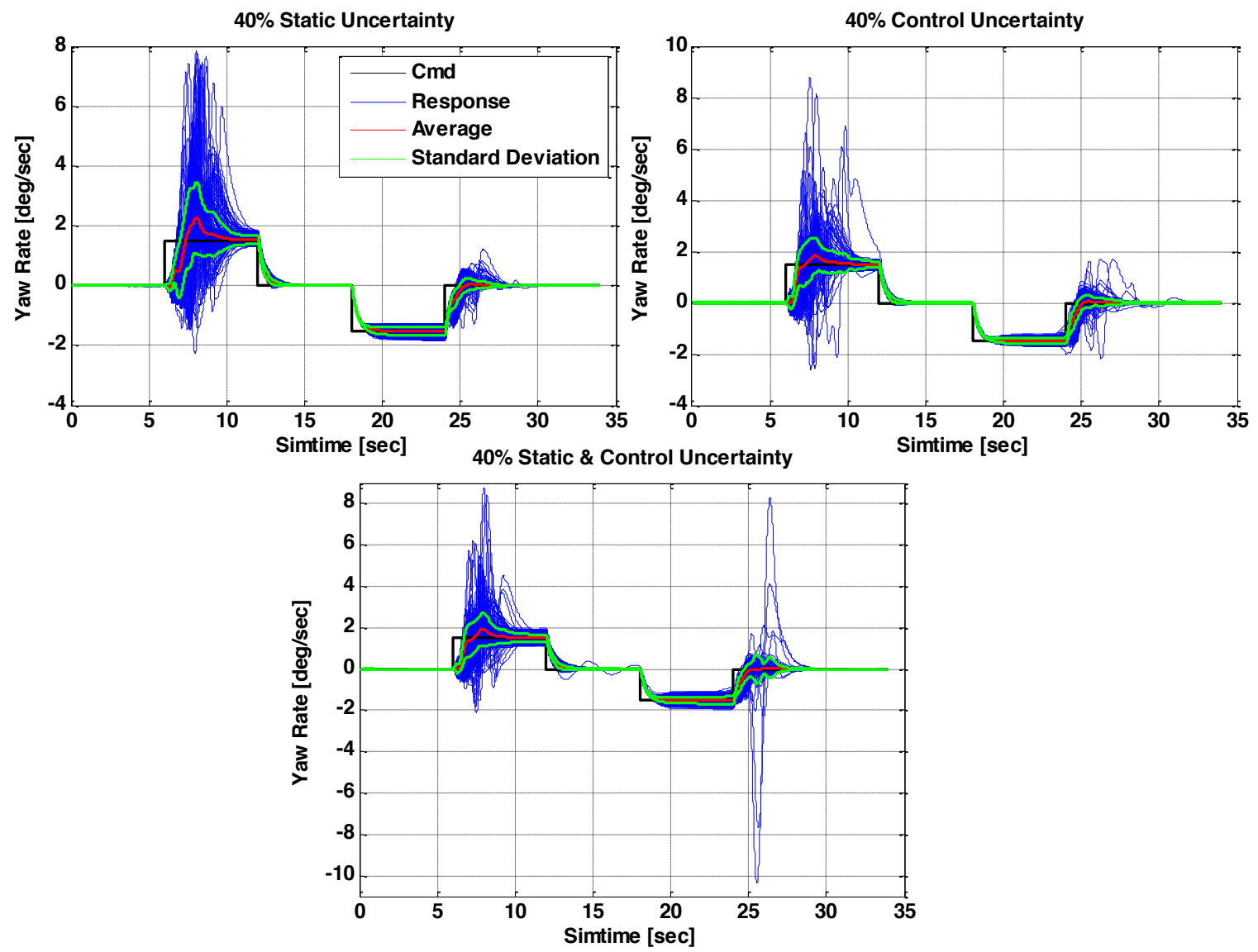

Figure 3-18: Yaw rate response with 40\% plant uncertainty.

Oscillations caused by yaw axis saturation at the initial positive step input increases in magnitude for all uncertainty cases. The dynamic inversion controller takes longer to close the error on yaw rate after the overshoot between 5 and 10 seconds. The delay is generated by larger 
uncertainty errors causing more tracking errors in the roll and pitch axis, as well as the yaw axis. The controller tracks negative yaw step inputs at 12 seconds and 18 seconds without as many oscillations. Figure 3-19 plots the standard deviations from each uncertainty case for yaw rate doublet tracking with $40 \%$ uncertainty. Table 3-10 shows the maximum steady state error for those uncertainty cases.

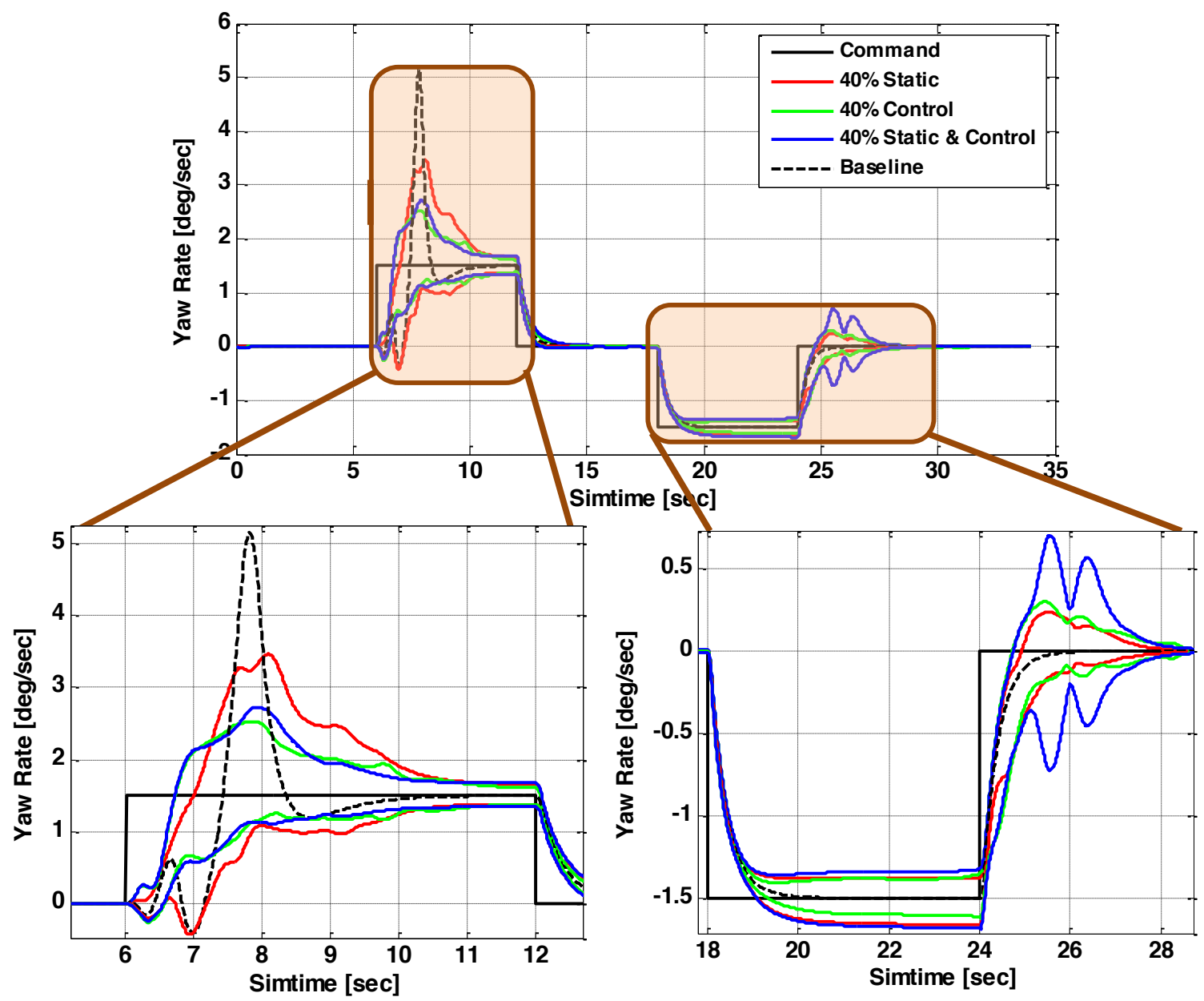

Figure 3-19: Standard deviation for yaw rate response with $40 \%$ plant uncertainty. 
Table 3-10: Maximum steady state error for yaw rate response with $40 \%$ plant uncertainty.

\begin{tabular}{|l|c|}
\hline \multicolumn{1}{|c|}{ Aerodynamic Uncertainty } & $\begin{array}{c}\text { Max Absolute } \\
\text { Steady State Error } \\
(\%)\end{array}$ \\
\hline \hline $40 \%$ Static Uncertainty & 64.5 \\
\hline $40 \%$ Control Uncertainty & 34.6 \\
\hline $40 \%$ Static \& Control Uncertainty & 30.2 \\
\hline
\end{tabular}

The standard deviation depicts the increased overshoot after the first positive step input and the increased oscillation magnitude after the second positive step input. The overshoots and oscillations at 24 seconds are more severe for the control uncertainty case and the static and control uncertainty case, compared to the static uncertainty case. They deviate farther from the baseline performance, which demonstrates control uncertainties degrade dynamic inversion controller tracking more than static uncertainties.

Figure 3-20 plots yaw rate doublet tracking for 500 simulation runs after inserting 50\% aerodynamic uncertainties. The corresponding standard deviation from each uncertainty case is depicted in Figure 3-21. 

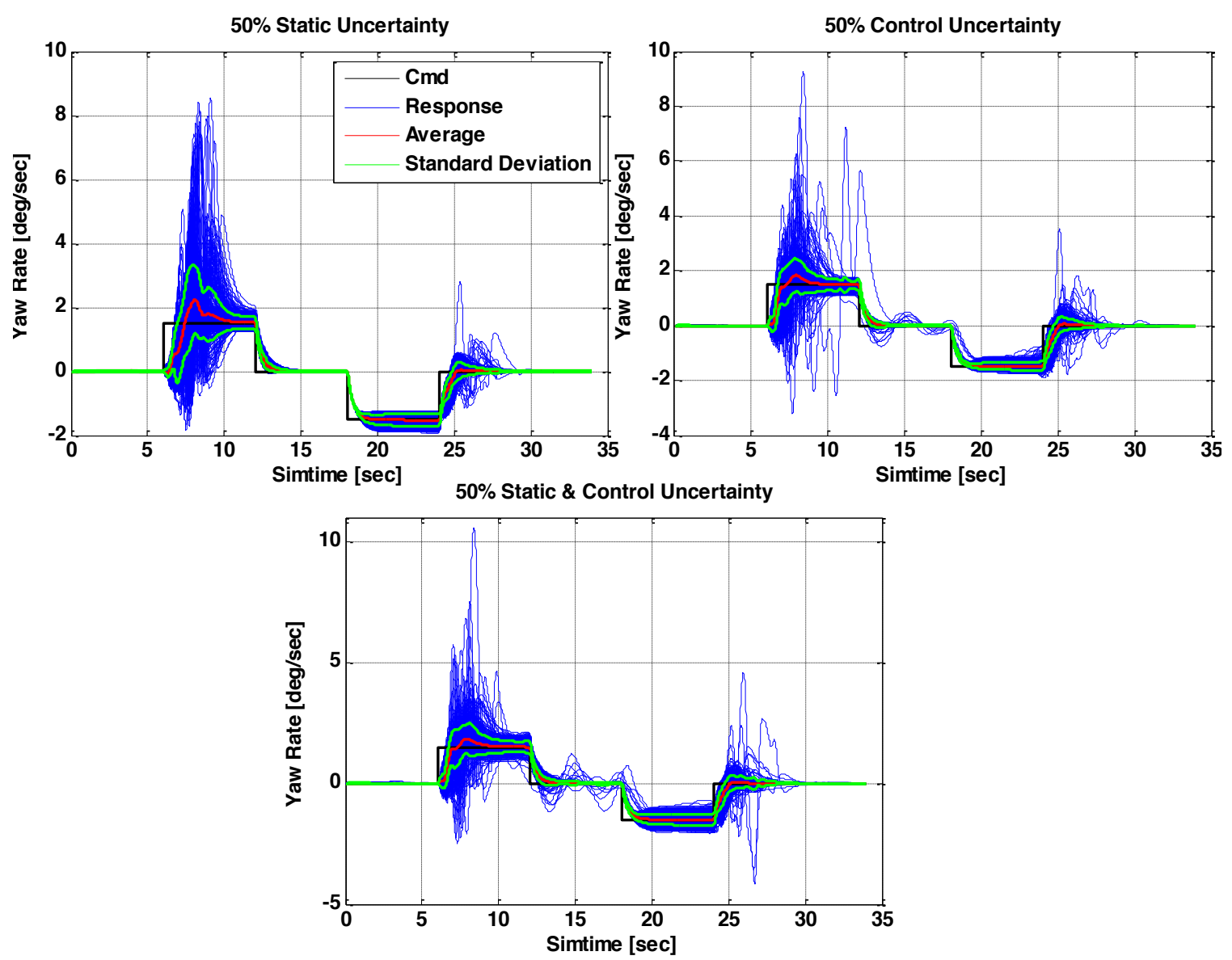

Figure 3-20: Yaw rate response with 50\% plant uncertainty.

Increasing uncertainty errors to $50 \%$ causes more oscillations for the control uncertainty case and the static and control uncertainty case. Negative yaw step input between 12 and 24 seconds induces oscillations about the steady state value, which is introduced after the control uncertainty increases. However, the static uncertainty case still demonstrates acceptable tracking with steady state errors close to zero degrees between 12 and 18 seconds. 


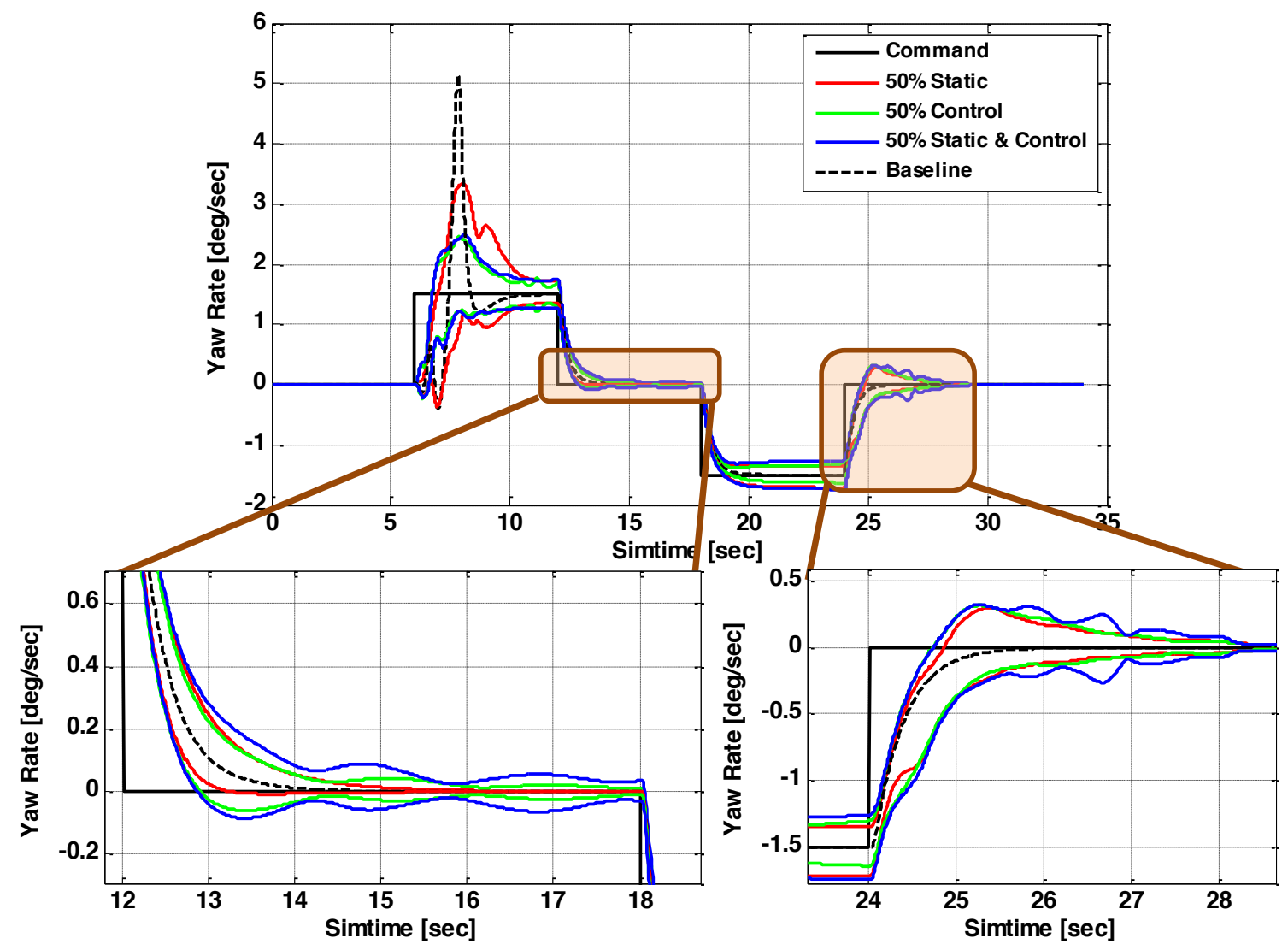

Figure 3-21: Standard deviation for yaw rate response with $\mathbf{5 0 \%}$ plant uncertainty.

Maximum steady state errors are computed in Table 3-11. The initial yaw rate overshoot at 8 seconds caused by the yaw axis saturation increases for the static uncertainty case, producing the largest steady state error out of all three uncertainty cases. The vehicle tracks yaw rate commands within acceptable limits as long as axis saturation has not occurred for the static uncertainty case. The standard deviation for the control uncertainty case and the static and control uncertainty case does not generate as much steady state errors, but increased errors between the plant and controller model initiates undesirable oscillations. 
Table 3-11: Maximum steady state response for yaw rate doublet with 50\% plant uncertainty.

\begin{tabular}{|l|c|}
\hline \multicolumn{1}{|c|}{ Aerodynamic Uncertainty } & $\begin{array}{c}\text { Max Absolute } \\
\text { Steady State Error } \\
(\mathbf{\%})\end{array}$ \\
\hline \hline $50 \%$ Static Uncertainty & 75.8 \\
\hline $50 \%$ Control Uncertainty & 28.5 \\
\hline $50 \%$ Static \& Control Uncertainty & 34.0 \\
\hline
\end{tabular}

The yaw rate overshoots and oscillations increases, as expected, when plant perturbations were increases to $60 \%$. Figure 3-22 depicts the dynamic inversion controller tracking for 600 simulation runs for the three uncertainty cases.

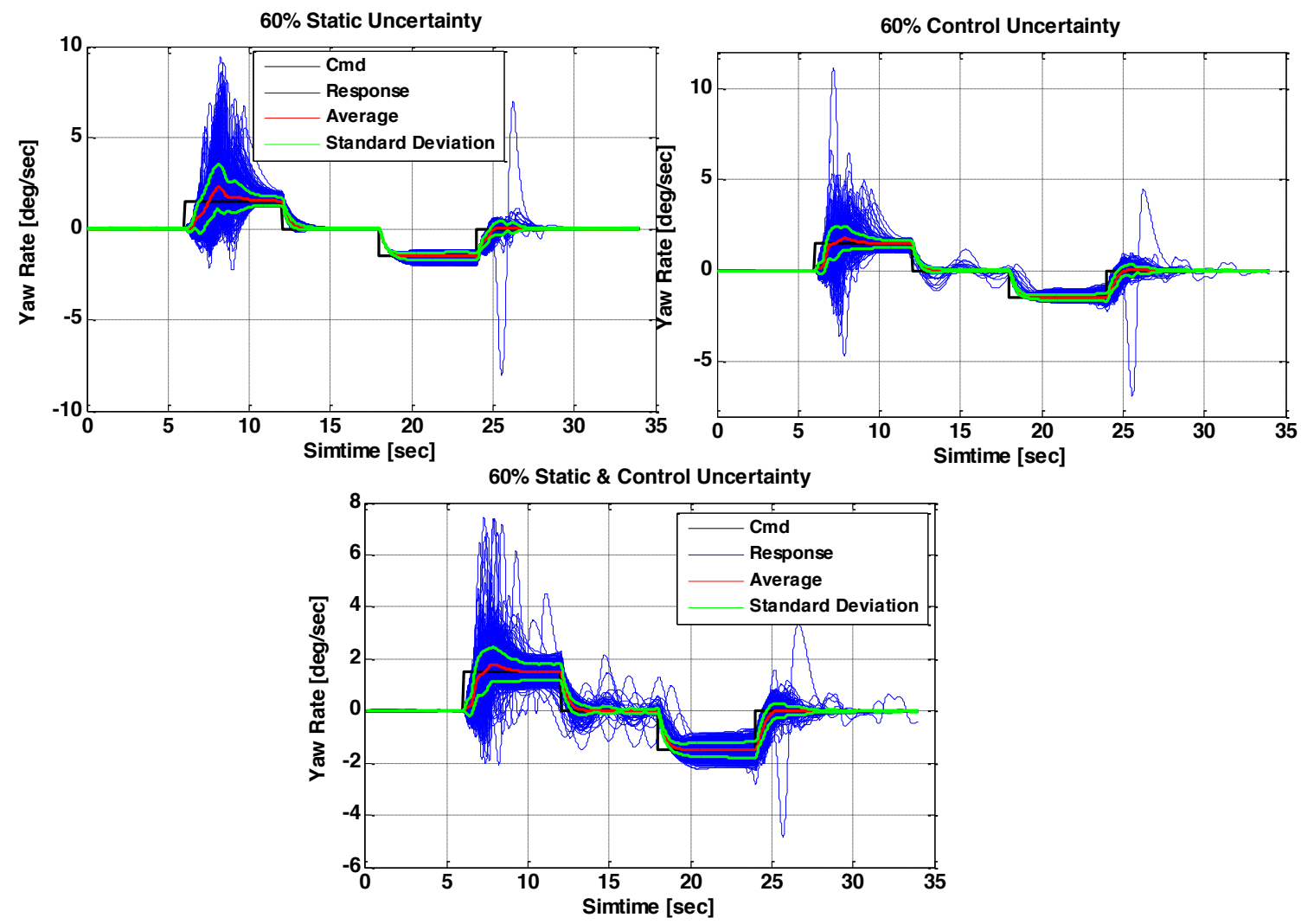

Figure 3-22: Yaw rate doublet response with $60 \%$ plant uncertainty. 
The standard deviation for each case captured in Figure 3-23 shows that even if the oscillation magnitudes are greater, the standard deviation performance did not vary much from the $50 \%$ case.

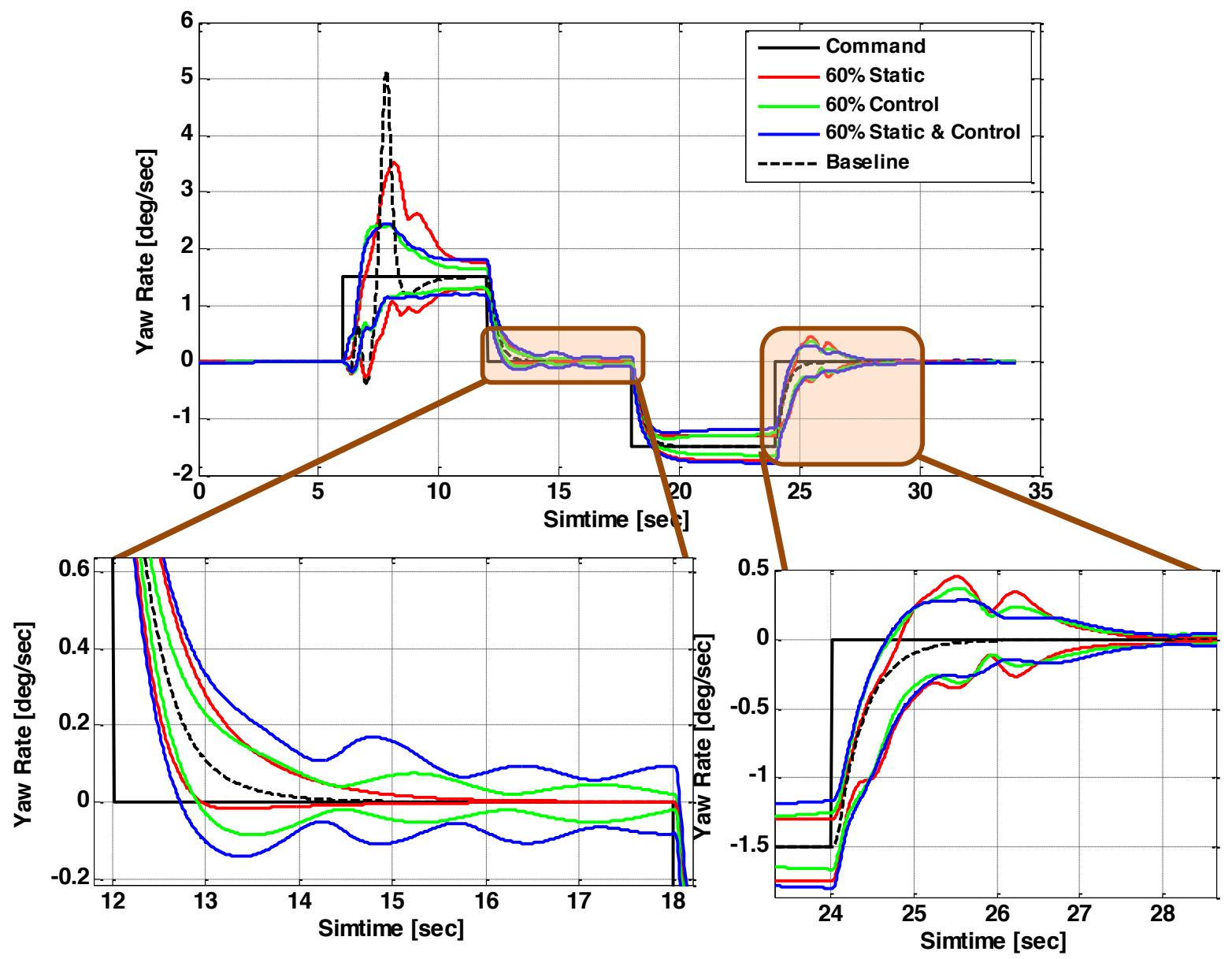

Figure 3-23: Standard deviation for yaw rate responses with $60 \%$ plant uncertainty.

The static uncertainty case shows the integral term reduces the yaw rate error close to zero degrees even with static aerodynamic errors of $60 \%$ when $\beta$ stays constant, between 14 and 18 seconds. The controller can track yaw rate commands with minimal error as long as the inherent plant dynamics stays constant. However, when innate plant dynamics change with $\beta, \alpha$, or Mach the controller cannot track commands without steady state errors, as demonstrated between 18 and 24 seconds when yaw rate command is non-zero resulting in changes in $\beta$. 
The control uncertainty case is more sensitive to aerodynamic errors between the plant and the controller. Larger oscillations are induced that are not present in the static uncertainty case between 14 and 18 seconds seen in Figure 3-23 when the uncertainty increases to $60 \%$. The control uncertainty drives the tracking performance of the static and control uncertainty case. Similar oscillations are observed in the static and control uncertainty case where the controller is causing the vehicle to become more unstable. Oscillations for a negative step input at 12 seconds and a positive step input at 25 seconds also increases. Aerodynamic errors degrades tracking performance for roll and yaw axis due to axis coupling arising from inexact plant dynamic cancellation. Overshoots in yaw rate occurs when uncertainties causes roll and pitch rate to overshoot more since less control authority is given to the yaw axis to achieve desired dynamics.

Maximum steady state error taken from the standard deviation proves the controller is still unacceptable for yaw rate tracking as seen in Table 3-12.

Table 3-12: Maximum steady state error for yaw rate response with $60 \%$ plant uncertainty.

\begin{tabular}{|l|c|}
\hline \multicolumn{1}{|c|}{ Aerodynamic Uncertainty } & $\begin{array}{c}\text { Max Absolute } \\
\text { Steady State Error } \\
(\%)\end{array}$ \\
\hline \hline $60 \%$ Static Uncertainty & 74.7 \\
\hline $60 \%$ Control Uncertainty & 28.5 \\
\hline $60 \%$ Static \& Control Uncertainty & 34.1 \\
\hline
\end{tabular}

The dynamic inversion controller is more sensitive to aerodynamic uncertainties in the yaw axis compared to the roll and pitch axes. Static and control uncertainty should be less than $30 \%$ in order for the controller to meet acceptable tracking performance. The robustness may increase if a different control allocation method is employed. The control effectiveness matrix at the flight condition chosen to initialize the yaw doublet is close to singular, which causes control saturation when the pseudo inverse solution is used. However, an optimal control allocation scheme using a linear programmer may solve for a different set of control deflections to achieve 
desired dynamics within actuator limits, eliminating control saturations caused by taking a pseudo inverse and not from lack of control power.

\subsection{Trajectory Tracking Robustness Analysis}

Tracking an approach and landing trajectory is more of a realistic analysis of the robustness of the backstepping and dynamic inversion controller for the RLV. Previous doublet analysis is able to ignore the aspect of an unpowered vehicle's limited energy by freezing the flight condition. $\alpha, \beta, \mu$ and body rates are able to propagate, which does not affect that total energy of the vehicle because the velocity or the altitude never changes, only the vehicle orientation. However, propagating the vehicle's velocity and position vector will continuously decrease dynamic pressure, altitude, and velocity during an unpowered approach and landing condition. The loss of energy may affect the amount of control power that the vehicle could obtain as the flight condition changes through its landing trajectory. The trajectory from Section 1.7.3 provides the trajectory commands to guidance.

More than $2 / 3$ of the 300 simulation runs resulted in the RLV losing control when a $30 \%$ static uncertainty and control uncertainty was implemented into the plant model. Therefore, aerodynamic uncertainties of $10 \%$ are inserted and increased $10 \%$ at a time until the controller performance is deemed unacceptable by the figures of merit. Uncertainty errors cause the vehicle to touchdown at various times. For tracking analysis purposes, tracking errors are not calculated after the RLV reached touchdown at 0 feet. Vehicle states are clipped after touchdown and are not plotted after the touchdown point. The average and standard deviation for each uncertainty test cases are taken at every time step where there are vehicle state data available. 
Figure 3-24 shows the resulting altitude error and the $\gamma$ profile for 300 simulations for three uncertainty cases. The altitude error is computed by subtracting the vehicle altitude from the trajectory altitude command at the same mission elapsed time. Thus, a negative altitude corresponds to the vehicle flying at a higher altitude than the commanded trajectory. The backstepping controller computes $\gamma$ command after summing the trajectory $\gamma$ command and the corrective $\gamma$ command from altitude error. However, Figure 3-24 displays $\gamma$ command as the generated trajectory command and does not include the corrective $\gamma$ command in order to keep the plot simple.

For all uncertainty cases, initial oscillations in altitude error coincide with initial oscillations in $\gamma$ caused by the RLV not being trimmed at the start of the simulation. Altitude error approaches zero feet around 63 seconds as the vehicle flies a steady state $\gamma$ during the steep glide slope phase. The circular pull up maneuver generates a change in $\gamma$ command as well as $\dot{\gamma}$ command resulting in altitude error oscillations when the controller tracks the $\gamma$ ramp with error present. Altitude error resembles any tracking error with $\gamma$ since the backstepping formulation uses $\gamma$ as the pseudo control of altitude. 

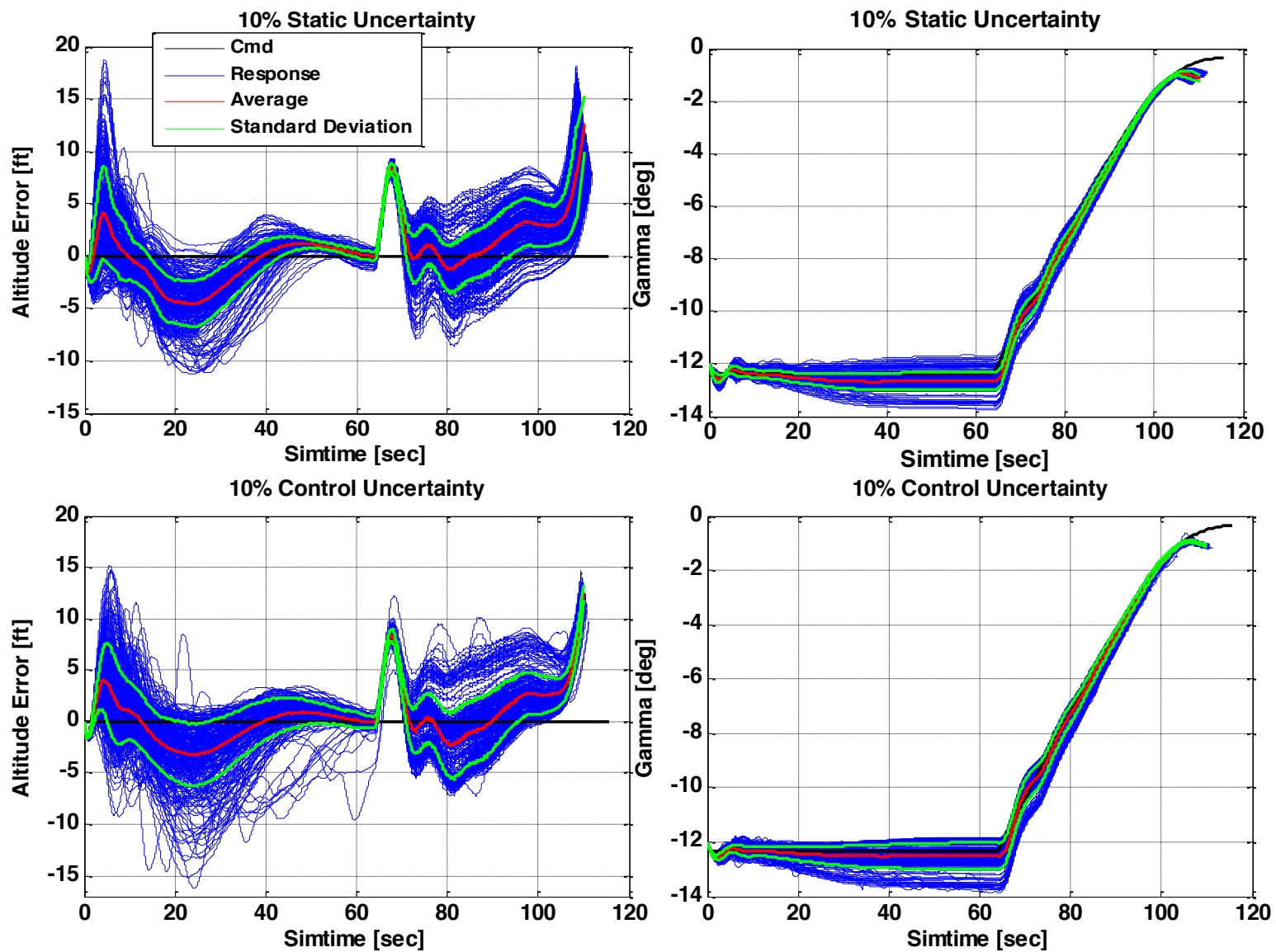

$10 \%$ Static \& Control Uncertainty
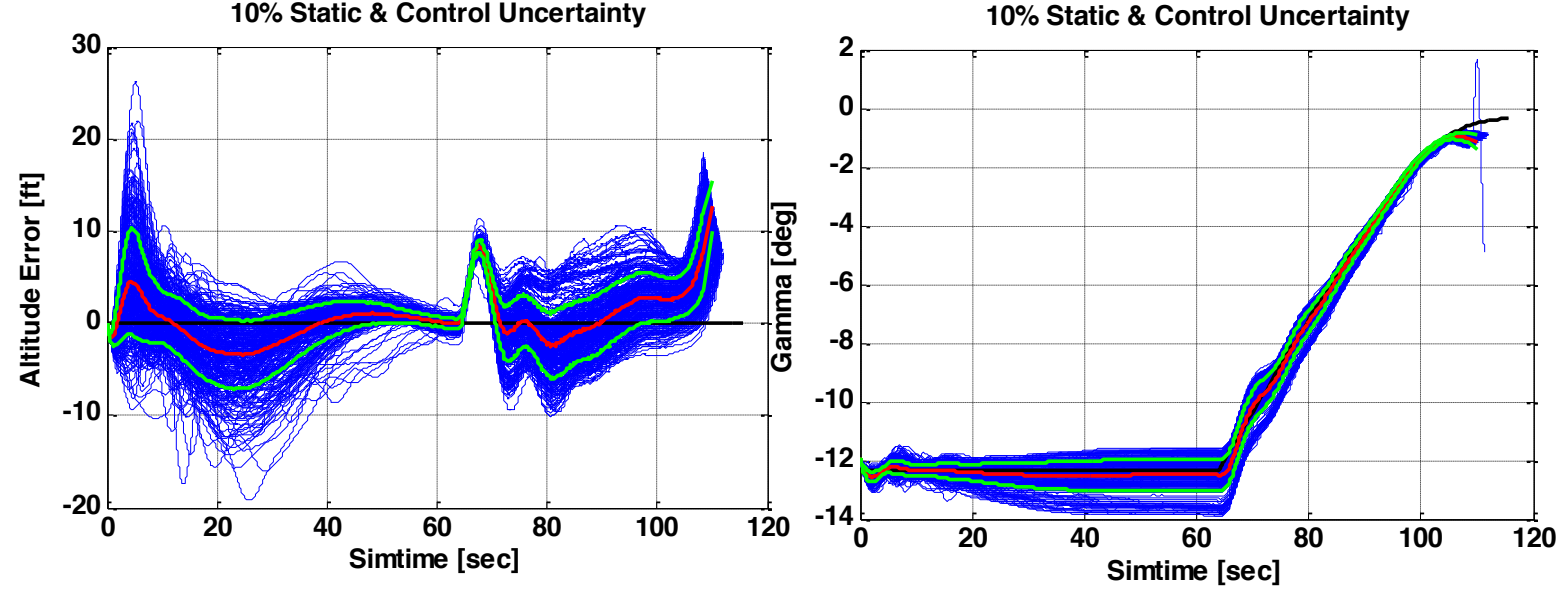

Figure 3-24: Altitude error and flight path angle profile with 10\% plant uncertainty.

More altitude error oscillations are observed in the control uncertainty case and the static and control uncertainty case during the steep glideslope phase due to the oscillations in tracking $\gamma$, as seen in Figure 3-24. $\gamma$ tracking is driven by $\alpha$ tracking. Control uncertainty causes $\alpha$ tracking 
to oscillate more than the static uncertainty case. $\alpha$ commands are determined by taking the pseudo inverse of the static lift curve slope after the control contributions to lift are subtracted out from the total desired lift. Static aerodynamics are only affected by the flight condition, $\alpha$, Mach, and $\beta$, thus, the static lift curve slope does not vary as much from each time step that the pseudo inverse solution is found since flight condition does not change as quickly. On the other hand, control aerodynamics are affected by flight conditions and control deflections. The control allocator solves for different control deflections for every time step if desired acceleration is not achieved resulting in lift contribution from control deflection to change more frequently than the static aerodynamic contribution. Therefore, control uncertainty causes more oscillations in $\alpha$ commands compared to the static uncertainty case, leading to oscillations in $\gamma$, directly affecting altitude error.

Vertical flight path angle implicitly controls the amount of energy dissipated by controlling the altitude and velocity. Figure 3-25 represents the $\gamma$ profile for the first 70 seconds of the trajectory during the steep glide slope phase, and the last 15 seconds of the trajectory when the vehicle touches down. A $\gamma$ than the trajectory in the steep glide slope phase causes the vehicle to maintain a higher altitude corresponding to a higher potential energy, which means more kinetic energy is being dissipated. Amount of kinetic energy dissipation directly relates to decreases in velocity. The vehicle may not have enough energy to maintain the proper $\alpha$ to fly the $\gamma$ profile to reduce altitude error if the vehicle dissipates too much energy, which occurs for one simulation run for the static and control uncertainty case. 


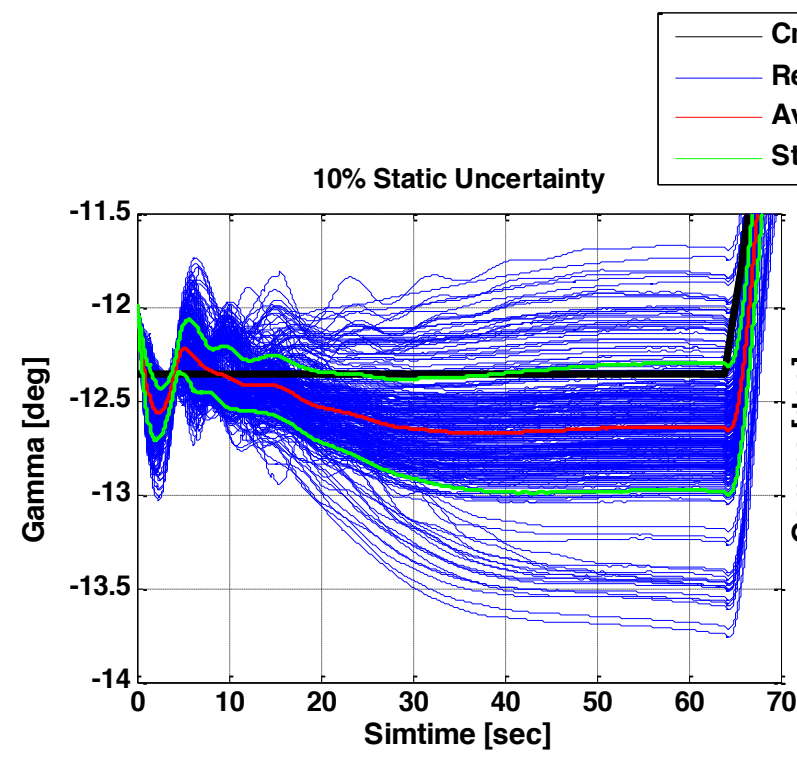

Cmd

Response

Average

Standard Deviation

$10 \%$ Static Uncertainty
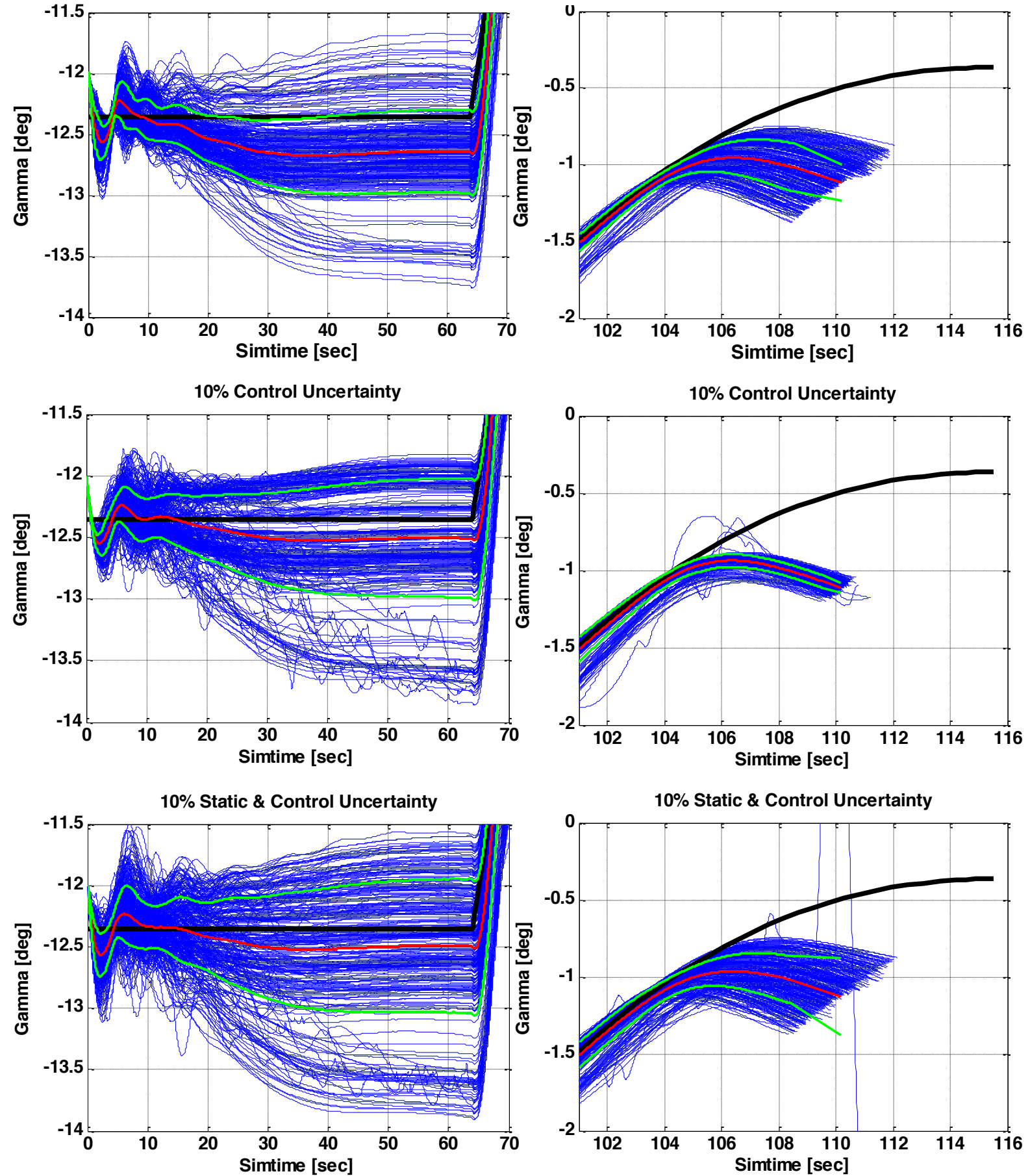

Figure 3-25: Zoomed in view of the flight path angle profile for $10 \%$ plant uncertainty. 
For all uncertainty cases, the controller flies a lower $\gamma$ profile the last 15 seconds of the landing trajectory. The uncertainty causes the vehicle to command a wrong $\alpha$ to track a $\gamma$ and altitude commanded from the backstepping controller. Uncertainty resulted in a vehicle achieving a lower $\gamma$, which corresponds to more kinetic energy and less potential energy compared to the trajectory, meaning the RLV is flying a lower altitude at a higher velocity. Thus, the vehicle reaches touchdown altitude quicker and at a faster sink rate than the generated trajectory. The higher sink rate is represented in Figure 3-26 depicting the sink rate for the last 15 seconds for each uncertainty case and the corresponding $\beta$ profile.

All uncertainty cases are unable to keep $\beta$ constant at zero degrees. There is always a slight $\beta$ that produces a small amount of drag, which contributes to kinetic energy dissipation. A slight $\beta$ will cause the vehicle to deviate from the generated trajectory since the trajectory was generated under the assumption that there are no drag contributions from $\beta$. Faster energy dissipation results in the RLV to touchdown between 3 and 7 seconds prior to the generated trajectory, as shown in Figure 3-26.

Control uncertainty and static and control uncertainty cases depict $\beta$ oscillations more frequently than the static uncertainty case. $\beta$ tracking is directly related to commanding the proper yaw rate command and tracking that yaw rate command with minimal error. The appropriate yaw rate is calculated with the correct calculation of lift, drag, and side force. As mentioned previously, static uncertainty is only affected by the flight condition, but control uncertainty is affected by control deflections in addition to flight conditions. Therefore, control uncertainty generates oscillations in $\beta$ when control deflections changes every time step and thereby causing fluctuations in force calculations. 

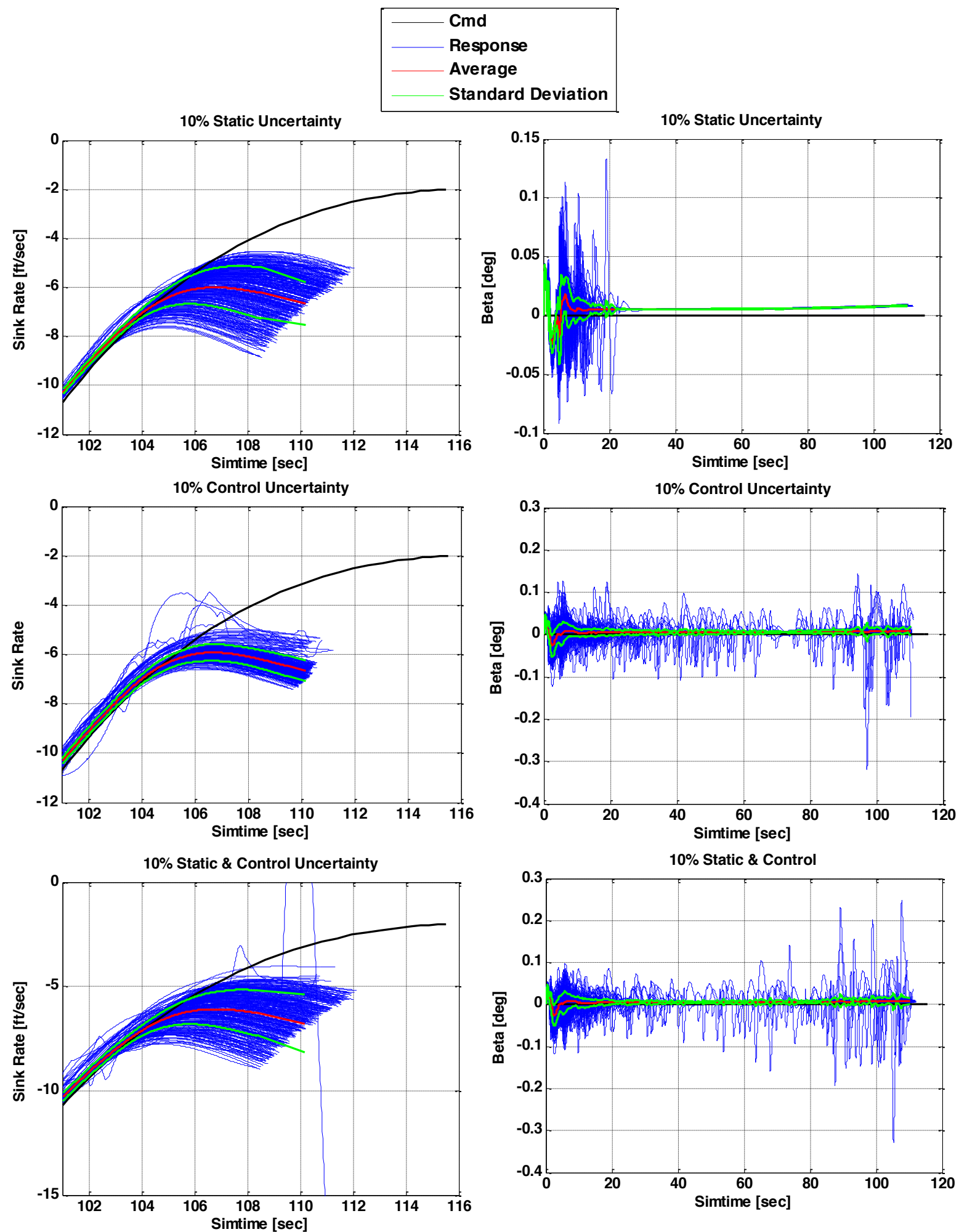

Figure 3-26: Sink rate at touchdown and side slip angle profile for $10 \%$ uncertainty. 
The oscillatory tracking of $\beta$ for the control uncertainty case and the static and control uncertainty case is seen again for $\chi$ tracking, as seen in Figure 3-27. $\mu$ is the pseudo control for $\chi$, and an accurate $\mu$ command relies on computing accurate aerodynamic forces which are mostly affected by the control uncertainty. $\chi$ is a pseudo control for crosstrack, so the resultant crosstrack performance from Figure 3-27 shows more oscillations for control uncertainty case and the static and control uncertainty case. The static uncertainty case is able to track the landing trajectory with the least amount of crosstrack error compared to cases with control uncertainty since $\chi$ tracking oscillates less.

Figure 3-28 compares the standard deviation for each uncertainty case for each parameter in the figures of merit: altitude error, sink rate at touchdown, $\chi$. The controller is capable of tracking the landing trajectory within acceptable limits with $10 \%$ uncertainty according to the figures of merit, excluding the one outlier case where the vehicle loses control right before touchdown in the static and control uncertainty case. The altitude error is within good tracking limits until the last couple seconds before touchdown when altitude errors are over 10 feet, corresponding to acceptable tracking. The sink rate for each uncertainty case is greater than $-5 \mathrm{ft} / \mathrm{sec}$, but less than $-10 \mathrm{ft} / \mathrm{sec}$ resulting in acceptable tracking. Good tracking corresponds to a crosstrack error within $+/-5 \mathrm{ft}$ and $\chi$ within $+/-5$ degrees of the runway $\chi$. Figure 3-28 shows the tracking performance for each uncertainty case are similar except the static uncertainty case has a smaller standard deviation. The standard deviation is less since static uncertainty does not cause as many oscillations when tracking the altitude profile or keeping crosstrack error close to zero. Table 3-13 evaluates controller tracking for each uncertainty case against the figures of merit, where green represents good tracking and yellow represents acceptable tracking. 


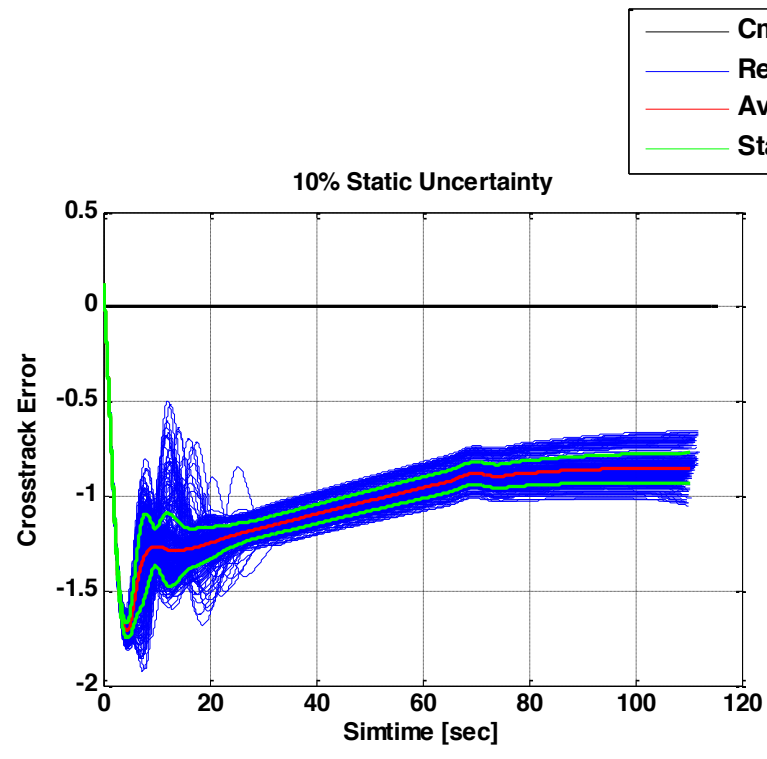

Cmd

Response

Average

Standard Deviation
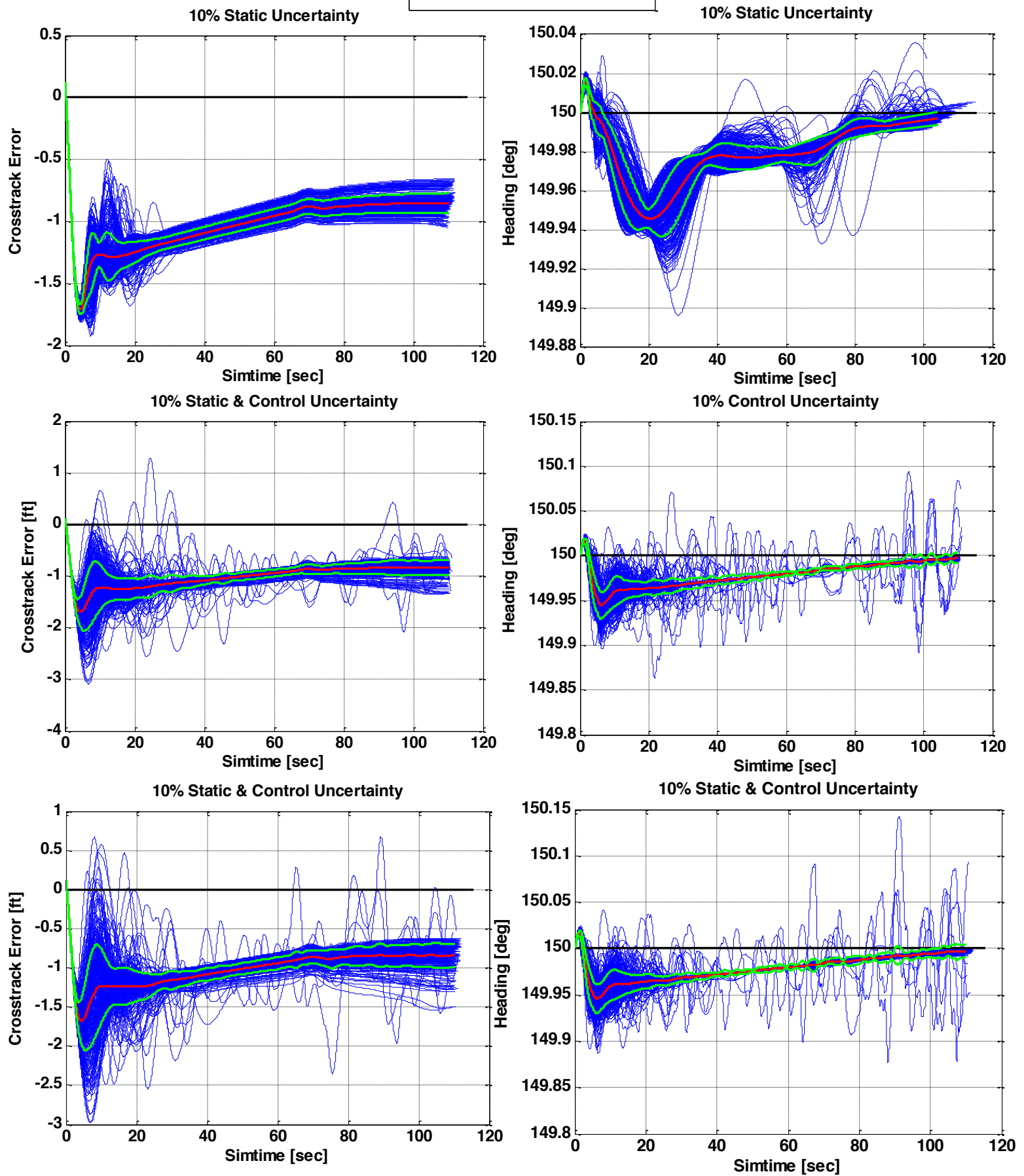

Figure 3-27: Crosstrack and heading profile for $10 \%$ uncertainty case. 

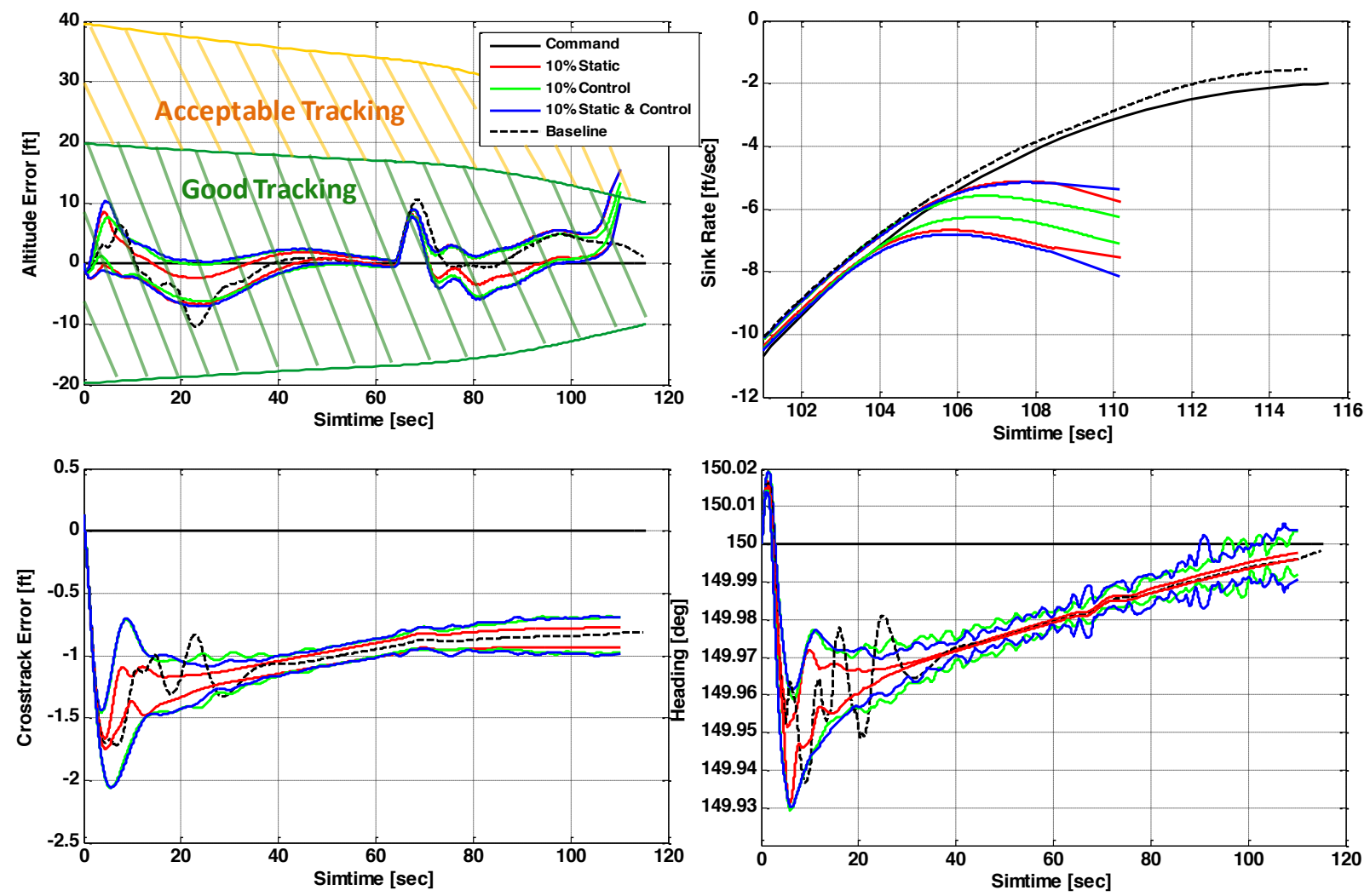

Figure 3-28: Standard deviation for altitude error, sink rate, crosstrack error, and heading for tracking a landing trajectory.

Table 3-13: Controller performance for the landing trajectory tracking with $10 \%$ plant uncertainty.

\begin{tabular}{|c|c|c|c|c|}
\hline Uncertainty Case & $\begin{array}{c}\text { Max } \\
\text { Crosstrack } \\
\text { Error (ft) }\end{array}$ & $\begin{array}{c}\text { Sink Rate at } \\
\text { Touchdown } \\
\text { (ft/sec) }\end{array}$ & $\begin{array}{l}\text { Heading } \\
\text { (deg) }\end{array}$ & $\begin{array}{c}\text { Max Altitude } \\
\text { Error (ft) }\end{array}$ \\
\hline $10 \%$ Static Uncertainty & -1.751 & -7.551 & 149.997 & 15.3 \\
\hline $10 \%$ Control Uncertainty & -2.061 & -7.098 & 150.0035 & 13.22 \\
\hline $10 \%$ Static \& Control Uncertainty & -2.059 & -8.157 & 150.004 & 15.14 \\
\hline
\end{tabular}

Plant perturbations increases to $20 \%$ for each uncertainty case. However, the static uncertainty and control uncertainty cases shows loss of control right before touchdown between four and seven simulations runs for each case. Figure 3-29 shows sink rate at touchdown and the corresponding $\gamma$ profile for $20 \%$ static uncertainty and control uncertainty cases. Control loss is 
observed when $\gamma$ drops resulting in an increase in sink rate. The controller is deemed unacceptable with $20 \%$ plant uncertainty since more than one simulation run shows the vehicle losing control. Aerodynamic uncertainty causes the vehicle to compute the wrong $\alpha$ command from $\gamma$ error. The feedback gains for $\gamma$ and $\alpha$ may have been too aggressive in the presence of aerodynamic modeling error and drove the system more towards instability causing oscillations in $\gamma$ and $\alpha$ tracking. Oscillations result in excessive energy dissipation causing the RLV to reach touchdown faster than the generated trajectory or lose control when the RLV cannot maintain $\alpha$.
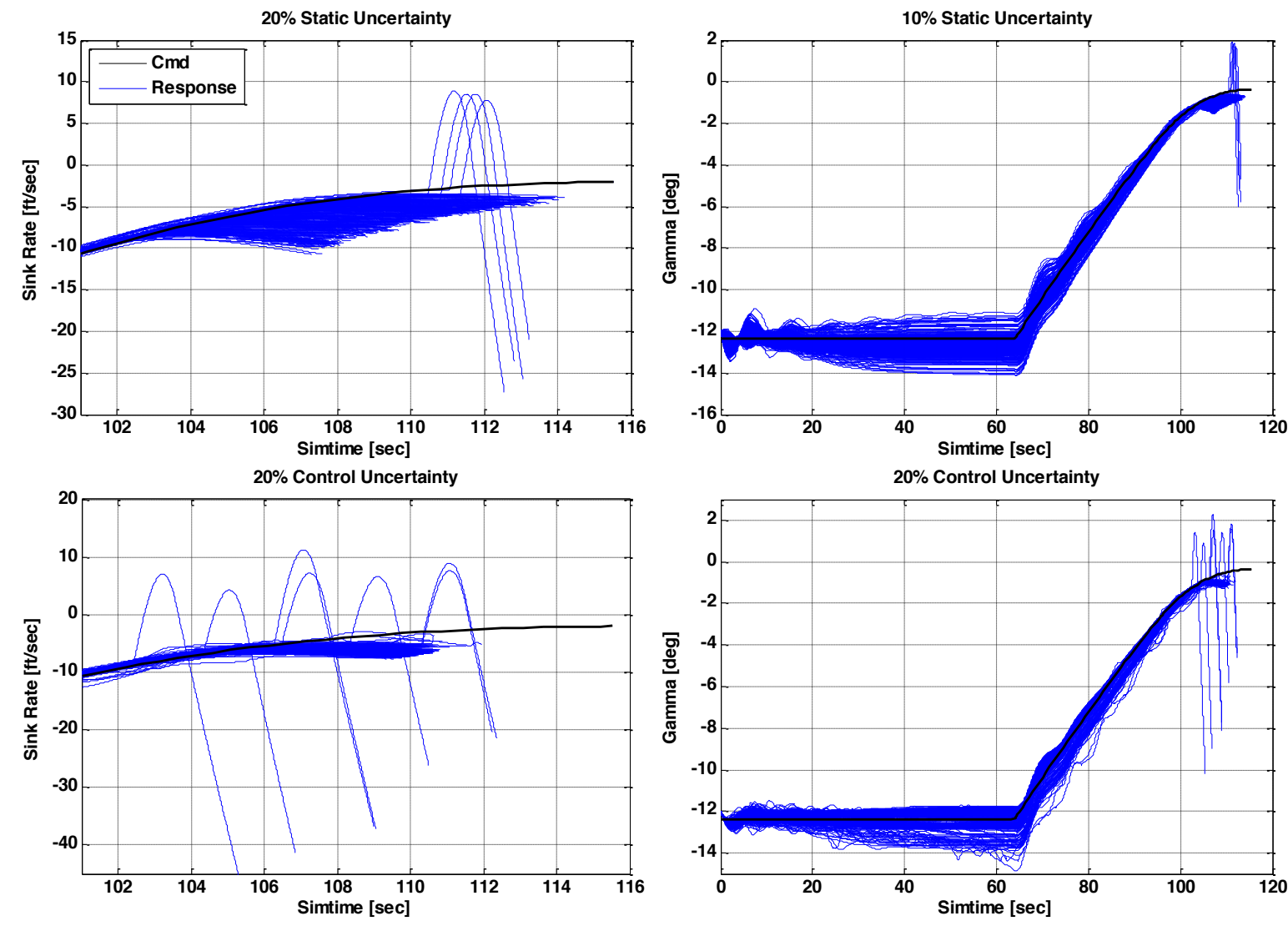

Figure 3-29: Sink rate at touchdown and flight path angle profile with $20 \%$ plant uncertainty 


\section{Conclusions and Future Work}

\subsection{Conclusion}

The Air Force has been working towards developing technology for operationally responsive space (ORS), which is the ability to launch military assets into space without the long set up time currently required. Part of the solution to ORS is to develop a reusable booster vehicle that could send any vehicle into orbit, then descend back to the atmosphere and land unpowered so that it would be capable of sending another vehicle up to space within a short period of time. This levies a requirement that the vehicle should be able to perform multiple sets of missions. Guidance and control are essential determining whether the vehicle will be able to complete its mission.

Classical control has proven to be a robust method to control the vehicle, but the gains have to be re-tuned for a different trajectory, which takes time. Nonlinear control methods like backstepping or dynamic inversion may eliminate the need to re-tune gains because it considers the dynamics of the vehicle that could be applied to the whole flight envelope. However, they are both model based methods that require accurate knowledge of the plant and may not be robust enough to handle uncertainties in the plant that comes from inaccurate aerodynamic analysis in hypersonic or transonic regions.

Therefore, the objective of this thesis was to develop a backstepping outer loop and dynamic inversion inner loop controller for a reusable launch vehicle configuration and evaluate its robustness characteristics by inserting aerodynamic uncertainties into the static and control surface aerodynamics data separately and then together. For each simulation run, the uncertainties 
were varied between each aerodynamic coefficient, but kept constant throughout all flight conditions. Simulation runs for each uncertainty case were determined based on the Law of Large Numbers, thus, dependent on the amount of uncertainty percentage variation. As aerodynamic uncertainty increased from $+/-30 \%$ to $+/-60 \%$, simulation runs for each uncertainty case increased from 300 simulation runs to 600 simulation runs. The theory behind the Law of Large Numbers states the average controller tracking could be determined if enough simulation runs were conducted for each uncertainty case. Simulation runs for each uncertainty percentage could be increased in order to increase the likelihood of encompassing all vehicle responses to the varying aerodynamic uncertainty.

The body rate doublet test cases showed that errors in control surface aerodynamics caused larger errors than the static aerodynamic uncertainty cases and drove the vehicle towards instability faster. The yaw axis was most sensitive to plant perturbation, while the roll axis was the most robust. The dynamic inversion controller was unable to track yaw rate commands within acceptable limits with $30 \%$ aerodynamic uncertainties; while the controller was robust to $60 \%$ static uncertainties and 50\% control uncertainties separately, and 50\% static and control uncertainties together in the roll axis. The pitch axis was able to handle $40 \%$ static uncertainty and 50\% control uncertainty when applied separately and 50\% static and control uncertainties.

Doublet test cases demonstrated that the dynamic inversion controller tracked body rate commands closer to the baseline with static uncertainties than control uncertainties. Control uncertainties affected control effectiveness matrix computation resulting in the control allocator to solve for control deflections that achieve more or less moment depending on the uncertainty error. The additional moment sometimes prevented actuator saturations when the vehicle could achieve a new flight condition with higher control effectiveness. For the static aerodynamic 
uncertainties, the vehicle was able to close the error without much overshoot or oscillations when the flight condition, $\alpha$, Mach, and $\beta$ stayed fairly constant. However, uncertainties in control surface aerodynamics sometimes resulted in oscillations in vehicle response even when flight conditions were constant because moment contributions from control surfaces vary as the deflections change. Control surface deflections were constantly being recomputed by the dynamic inversion inner loop causing the allocator to change control deflection commands leading to body rate oscillations. The benefit of using dynamic inversion was that it was formulated so that it decouples the roll, pitch, and yaw axis as long as the dynamics were modeled accurately. The body rate dynamics become a bank of decoupled integrators. However, when uncertainties were introduced into the plant model, the controller was unable to accurately model and cancel the plant dynamics, which re-introduced the axes coupling inherent in the plant. The coupling caused the performance in one axes to degrade if the other axes diverged from their commands.

Tracking a landing trajectory presented a method to evaluate the dynamic inversion controller with the backstepping outer loop controller. Previously for the dynamic inversion robustness analysis, infinite amount of energy was provided to the vehicle by freezing the flight condition and only allowing body rates, $\alpha, \beta$, and $\mu$ to vary while holding altitude and velocity constant. Realistically, the RLV has a limited amount of energy since the vehicle is unpowered during an approach and landing configuration. The limited amount of energy affected trajectory tracking when degraded controller performance from aerodynamic uncertainties cause excessive energy dissipation. Limiting the vehicle's energy by propagating its position vector reduced the amount of plant perturbation the controller could handle without losing control. The controller could only handle $10 \%$ aerodynamic uncertainties. Uncertainties above $20 \%$ caused the vehicle to dissipate too much energy before the final pull up maneuver resulting in the vehicle to lose control when $\alpha$, corresponding to the desired $\gamma$, could not be maintained. Like the body rate 
doublet cases, control uncertainties caused more oscillations in tracking $\alpha$ command. More altitude, $\gamma$, and $\alpha$ oscillations were caused by control uncertainties compared to static uncertainties since moment and force contributions were affected by control deflections as well as flight conditions. Control deflections changes more frequently than flight conditions resulting in control dependent moment and forces variations causing tracking oscillations. Altitude, $\gamma$, and $\alpha$ feedback gains chosen for the baseline controller was not robust enough to tolerate $20 \%$ aerodynamic uncertainties. The gains were too aggressive, driving the vehicle towards instability.

\subsection{Future Work}

Future work can be done to improve the control allocation method for the dynamic inversion controller. Using a pseudo inverse solution for an underdetermined system does not provide a unique solution, or a bounded solution within actuator limits. At times, when the control effectiveness matrix was close to singular the pseudo inverse solution commanded actuator solutions beyond actuator limits causing the body axis to saturate. Moreover, the pseudo inverse solution caused control surface saturation even if enough control power existed because actuator limits were never considered when solving for control deflections. There are linear programming methods that can be employed to help reduce the problem of control surface saturation by including actuator limits within the allocation formulation. The same robustness analysis can be applied to test whether robustness to plant perturbation increases with an improved control allocator.

Trajectory tracking with plant uncertainties demonstrated nominal gains could be too aggressive in the presence of aerodynamic errors. An adaptive method can be applied to determine proper gains during runtime in order to prevent the vehicle from reaching instability. The nominal gains can be multiplied by a factor of axis saturation or tracking error to prevent 
integrator windup. Robustness may increase if gains are reduced when aerodynamic uncertainties caused altitude, $\gamma$, and $\alpha$ oscillations. 


\section{Bibliography}

1. AlSwailem, S. I. (2004). Application of Robust Control in Unmanned Vehicle Flight Control System Design. Cranfield, England: Cranfield University.

2. Balas, G. J. (1995). Design of a Flight Control System for a Highly Maneuverable Aircraft Using Robust Dynamic Inversion. Journal of Guidance, Control and Dynamics, 18-24.

3. Bang, H., Hurtado, J. E., \& Lian, B. (Aug. 2004). Adaptive Backstepping Control Based Autopilot Design for Reentry Vehicle. AIAA Guidance, Navigation, and Control Conference and Exhibit. Providence, Rhode Island.

4. Bergin, C. (2006 4-January). X-33/Venture Star. Retrieved 2010 йил 19-December from NASA Spaceflight: http://www.nasaspaceflight.com/2006/01/x-33venturestar-what-reallyhappened/

5. Blake, W. B., Ngo, A. D., Moster, G. E., \& Oppenheimer, M. W. (2006). A Rapid Assessment Tool For Space Access Vehicle Configurations in Guidance and Control Performance. Wright-Patterson Air Force Base, OH: Air Force Research Laboratory.

6. Borkowski, K. (1989). Accurate Algorithms to Transform Geocentric to Geodetic Coordinates. Bull. Geod. , 50-56.

7. Calise, A. J., Curry, M. D., \& Johnson, E. N. (2006). Adaptive Guidance and Control for Autonomous Hypersonic Vehicles. Journal of Guidance, Control, and Dynamics , 29 (3).

8. Chu, Q., da Costa, R., \& Mulder, J. (2003). Reentry Flight Controller Design Using Nonlinear Dynamic Inversion. Journal of Spacecraft and Rockets , 40 (1).

9. Clark, S. (2010 22-April). Atlas Rocket Delivers Air Force Spaceplane to Orbit. Retrieved 2010 29-September from Spaceflight Now:

http://spaceflightnow.com/atlas/av012/100422launch/

10. David, L. (2009 15-October). Reusable Rocket Plane Soars in Test Flight. Retrieved 2010 19December from Space.com:

http://www.msnbc.msn.com/id/33329828/ns/technology_and_science-space/

11. Doggrell, L. (2006 1-June). Operationally Responsive Space: A Vision for the Future of Military Space. Retrieved 2010 19-December from Air \& Space Power Journal: http://www.airpower.maxwell.af.mil/airchronicles/apj/apj06/sum06/doggrell.html

12. Doman, D. B., \& Oppenheimer, M. W. (2001 August). Improving Control Allocation Accuracy for Nonlinear Aircraft Dynamics. Retrieved 2009 14-April from DTIC Online: http://handle.dtic.mil/100.2/ADA405544

13. Doman, D. O. (2003). Reconfigurable Inner Loop Control of a Space Maneuvering Vehicle. AIAA Guidance, Navigation, and Control Conference and Exhibit. Austin, Texas.

14. Etkin, B. (1972). Dynamics of Atmospheric Flight. New York: John Wiley \& Son, Inc. 
15. Gandhi, N., Ward, D. G., \& Schierman, J. D. (2004). Integrated Adaptive Guidance and Control for Re-Entry vehicles with Flight-Test Results. Journal of Guidance, Control, and Dynamics , 27 (6).

16. Georgie, J., Daigoro, I., Valasek, J., \& Ward, D. (2002). Reentry Vehicle Flight Controls Design Guidelines: Dynamic Inversion. Houston, Texas: NASA.

17. Good, M. C. (2001 1-April). Sontags Formula Backstepping Control. Retrieved 2009 15December from Advanced Control and Automation: http://people.eng.unimelb.edu.au/mcgood/436-459/backstepping/Backstepping2Basic_backstepping.pdf

18. Hanson, J. M. (2000). Advanced Guidance and Control Project for Reusable Launch Vehicles. Proceedings of the AIAA Guidance, Navigation and Control Conference and Exhibit (AIAA Paper 2000-3957).

19. Harkegard, O. (2001). Flight Control Design Using Backstepping. Linkoping, Sweden: Linkopings Universitet.

20. Harkegard, O., \& Glad, S. T. (2000). A Backstepping Design for Flight Path Angle Control. Linkoping, Sweden: Division of Automatic COntrol Department of Electrical Engineering.

21. Ito, D., Valasek, J., \& Ward, D. T. (2001). Robust Dynamic Inversion Controller Design and Analysis for the X-38. AIAA Guidance, Navigation, and Control Conference and Exhibit. Montreal, Canada.

22. Joint Technology Office on Hypersonics . (2008 February). Roadmap for the Hypersonics Programs of the Department of Defense. Retrieved 2010 19-December from Department of Defense:

http://www.dod.gov/ddre/doc/Feb08_JTOH_Congressional_Public_Release_Approved.pdf

23. Joseph, A., \& Geetha, S. (2007 November). Application of Backstepping for the Control of Launch Vehicle. Retrieved 2009 21-December from The Institution of Engineers India: http://www.ieindia.org/pdf/88/88AS203.pdf

24. Kalviste, J. (1987). Spherical mapping and Analysis of Aircraft Anlges for Maneuvering Flight. AIAA Atmospheric Flight Mechnaics Conference, 24. Williamsburg, VA.

25. Kelso, T. (1995 September). Orbital Coordinate Systems, Part I. Retrieved 2009 27-August from Satellite Times: http://celestrak.com/

26. Kennedy Space Center. (n/a). Shuttle Discovery STS-133. Retrieved 2010 19-December from Kennedy Space Center Visitor Complex: http://www.kennedyspacecenter.com/event.aspx?id=53dd9501-2238-4255-ae6ea7562613d402

27. Kluever, C. (2004). Unpowered Approach and Landing Guidance Using Trajectory Planning. AIAA Guidance, Navigation, and Control Conference and Exhibit. Providence, Rhode Island.

28. Krebs, G. (2010 17-December). X-37B. Retrieved 2010 27-December from Sky Rocket: http://www.skyrocket.de/space/doc_sdat/x-37.htm 
29. Lewis, F. L. (2003). Aircraft Control and Simulation. New Jersey: John Wiley \& Sons, Inc.

30. Messier, D. (2010 1-April). Air Force Wrestles With EELV Launch Costs as NASA Weighs Options. Retrieved 2010 19-December from Parabolic Arc:

http://www.parabolicarc.com/2010/04/01/air-force-wrestles-eelv-launch-costs-nasa-weighsoptions/

31. NASA. (2010 12-March). A Countdown of Countdowns: The Space Shuttle's Finale. Retrieved 2010 19-December from Space Shuttle: http://www.nasa.gov/mission_pages/shuttle/behindscenes/shuttle_countdowns.html

32. NASA. (n.d.). Historical Fact Sheet: X-33 Advanced Technology Demonstrator. Retrieved 2010 19-December from Marshall Space Flight Center: http://www.nasa.gov/centers/marshall/news/background/facts/x33.html

33. NASA. (2002 April). The Space Launch Initiative: Technology to pioneer the space frontier. Retrieved 2009 19-December from Marshal Space Flight Center: http://www.nasa.gov/centers/marshall/news/background/facts/slifactstext02.html

34. NASA. (1999 October). X-34: Demonstrating Reusable Launch Vehicle Technologies. Retrieved 2010 19-December from Marshall Space Flight Center: http://www.nasa.gov/centers/marshall/news/background/facts/x-34.html

35. NASA. (2003 September). X-37 Technology Demonstrator: Blazing the Trail for the Next Generation of Space Transportation Systems. Retrieved 2010 27-December from NASA : www.msfc.nasa.gov/NEWSROOM/background/facts/x37-historical.pdf

36. Nelson, R. C. (1998). Flight Stability and Automatic Control. Boston, Massachusetts: McGraw-Hill Companies, Inc.

37. Norris, G. (2010 7-December ). Second X-37B Prepared For Launch. Retrieved 201027 December from Aviation Week:

http://www.aviationweek.com/aw/generic/story_channel.jsp?channel=space\&id=news/asd/20 10/12/07/02.xml\&headline=Second\%20X-37B\%20Prepared\%20For\%20Launch

38. Parsch, A. (2009 23-November). X-37/ X-40. Retrieved 2010 19-December from Designation Systems : Directory of U.S. Military Rockets and Missiles: http://www.designationsystems.net/dusrm/app4/x-37.html

39. Pike, J. (1999 14-January). Military Spaceplane X-40 Space Maneuver Vehicle Integrated Tech Testbed. Retrieved 2010 19-December from Space Policy Project Military Space Programs: http://www.fas.org/spp/military/program/launch/msp.htm

40. Pike, J. (2008 8-March). X-40 Space Maneuver Vehicle. Retrieved 2010 19-December from Glocal Security: http://www.globalsecurity.org/space/systems/x-40.htm

41. Sharma, M., \& Ward, D. G. (2002). Flight-Path Angle Control via Neuro-Adaptive Backstepping. AIAA .

42. The MathWorks, Inc. (2010). Overview of Model Referencing. Retrieved 2009 15-August from The MathWorks User's Guide: http://www.mathworks.com/help/toolbox/simulink 
43. U.S. Centennial of flight Commission. (n/a). Space Shuttle. Retrieved 2010 19-December from U.S. Centennial of flight Commission:

http://www.centennialofflight.gov/essay/SPACEFLIGHT/Shuttle/SP25.htm

44. Warwick, G. (2010 25-April). USAF Plans for Reusable Booster Development. Retrieved 2010 10-September from Aviation Week:

http://www.aviationweek.com/aw/generic/story.jsp?id=news/awst/2010/04/19/AW_04_19_2 $010 \_$p30-

219818.xml\&headline=USAF\%20Plans\%20For\%20Reusable\%20Booster\%20Development \&channel=defense 


\section{Appendix A: Approach and Landing Trajectory Propagation Scripts}

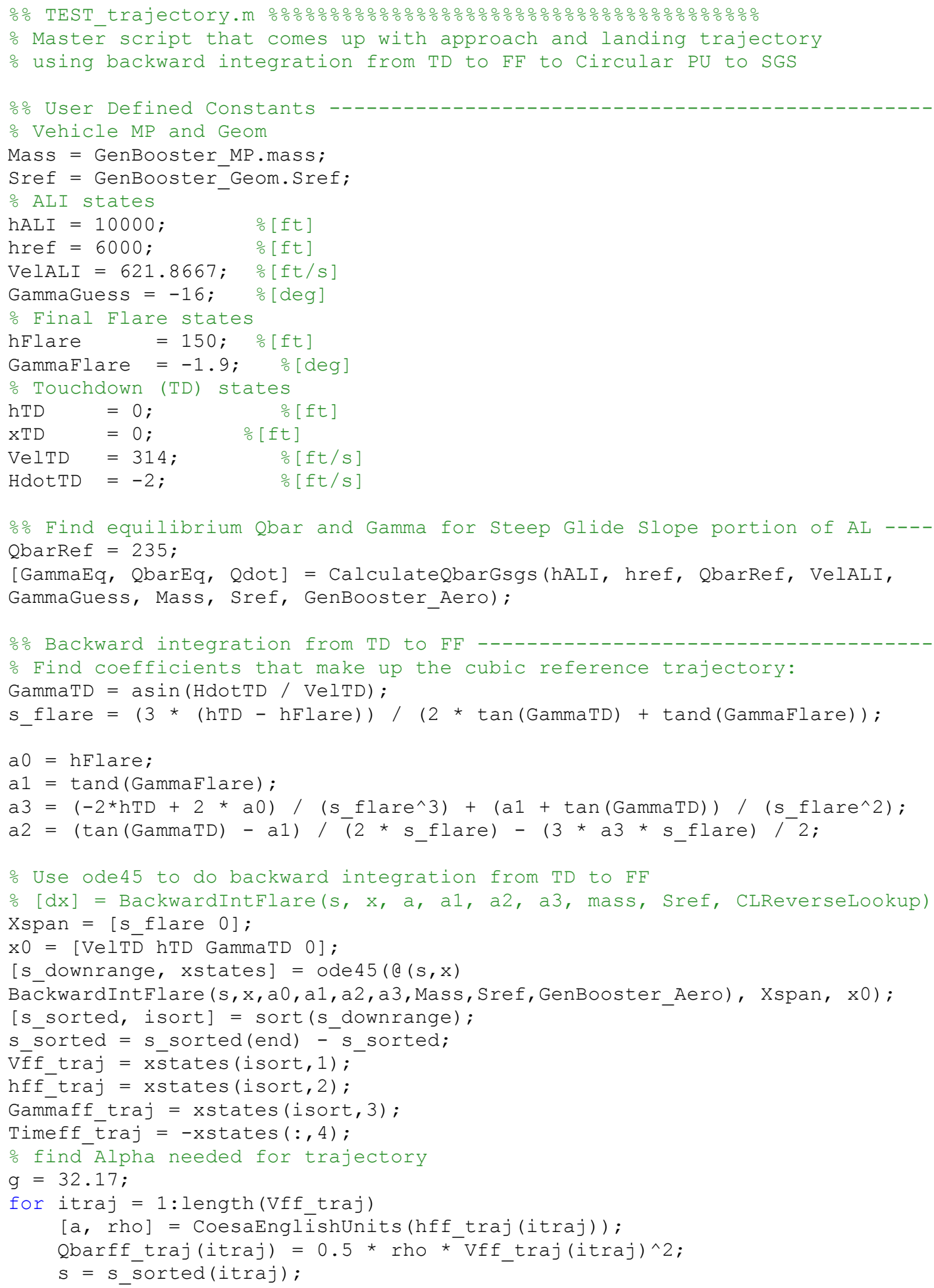




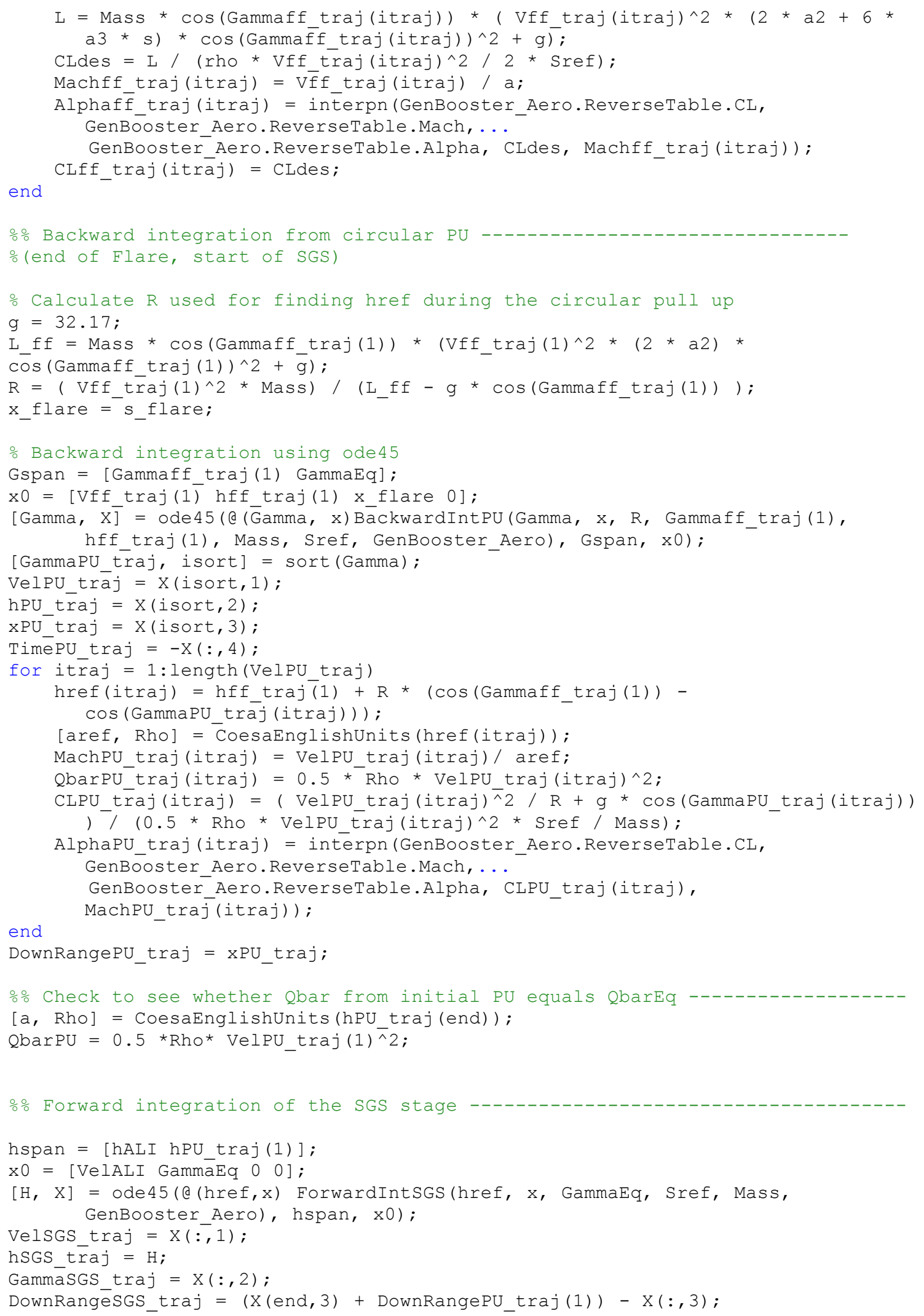




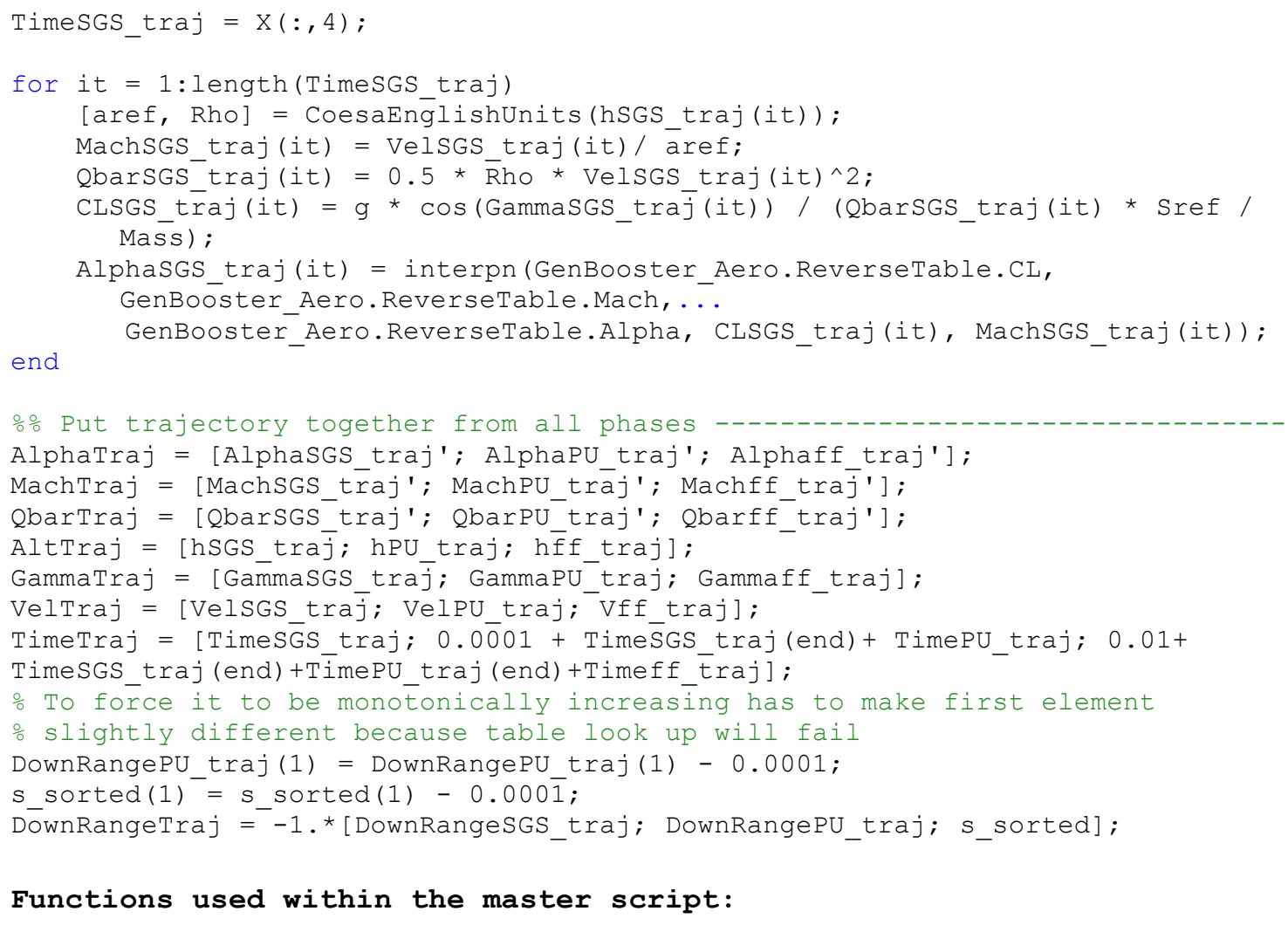

\section{Functions used within the master script:}

function [GammaEq, QbarEq, Qdot, iter,dQdotdG] = CalculateQbarGsgs (hALI, href, QbarRef, velAlI, GammaGuess, mass, Sref, GenBooster_Aero)

응 CalculateEqQbarGsgs ( ) .m

\% Calculates the equilibrium Qbar and Gamma_SGS that will obtain

o equilibrium Qbar for the steep glide slop phase of AL

$\div$ Inputs:

hALI [ft] ALI altitude

href [ft] Reference altitude used as the density to find equilibrium Qbar

GammaGuess [deg] Initial guess for equilibrium flight path 


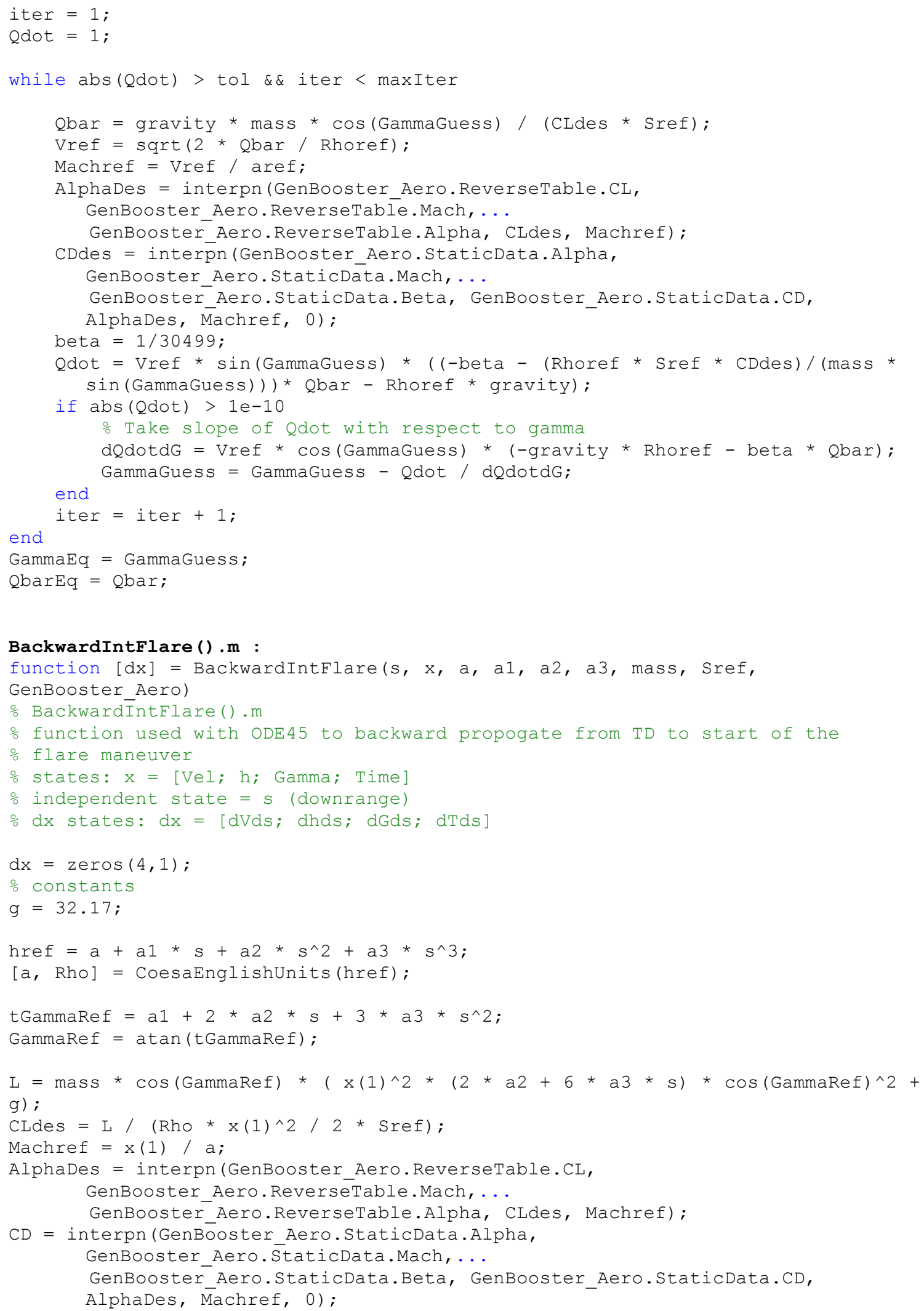

\section{BackwardIntFlare () .m :}

function $[d x]=$ BackwardIntFlare $(s, x, a, a 1, a 2$, a3, mass, Sref, GenBooster Aero) 


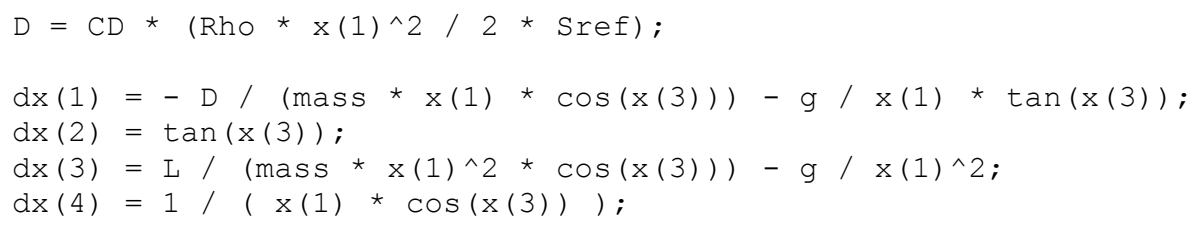

\section{BackwardIntPU () .m:}

function $[d x]$ = BackwardIntPU (Gamma, $x$, R, Gamma_ff, h_ff, mass, Sref, GenBooster_Aero)

\section{ForwardIntSGS ( ) .m:}

function [xdot] = ForwardIntSGS(href, x, GammaSGS, Sref, mass, GenBooster_Aero) $\circ \%$ ForwardintSGS 


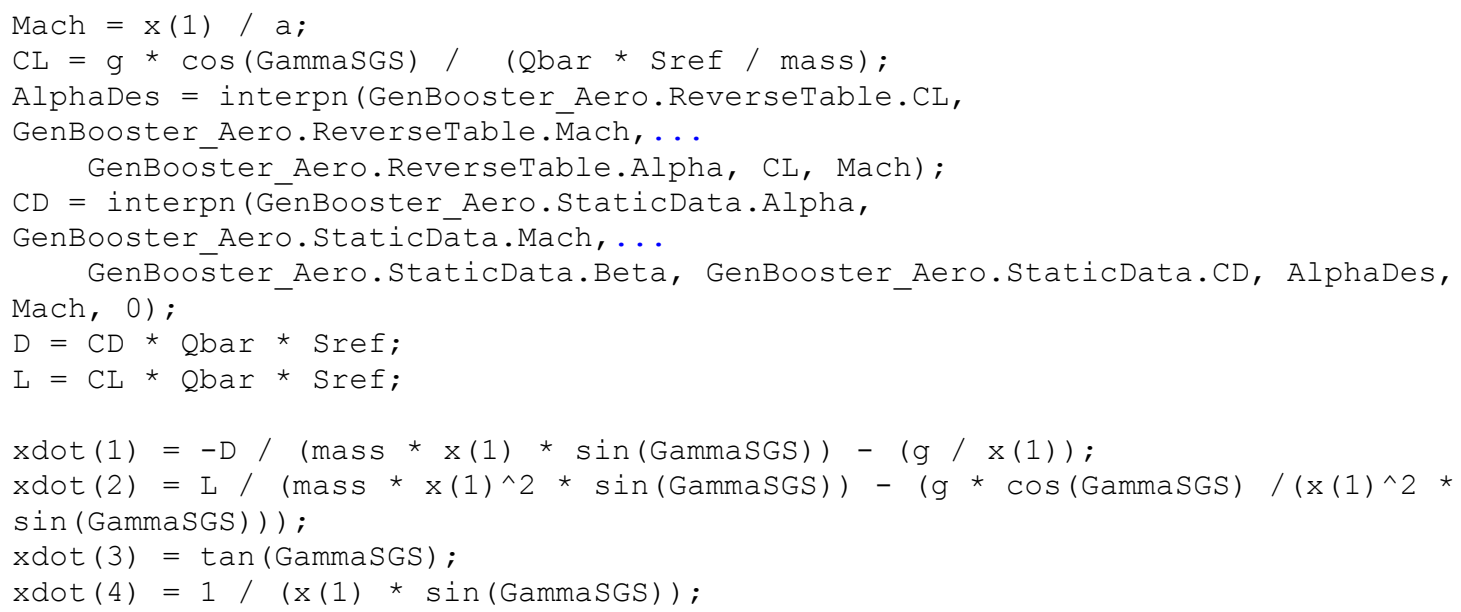




\section{Appendix B: Vincenty Function Used to Compute Crosstrack}

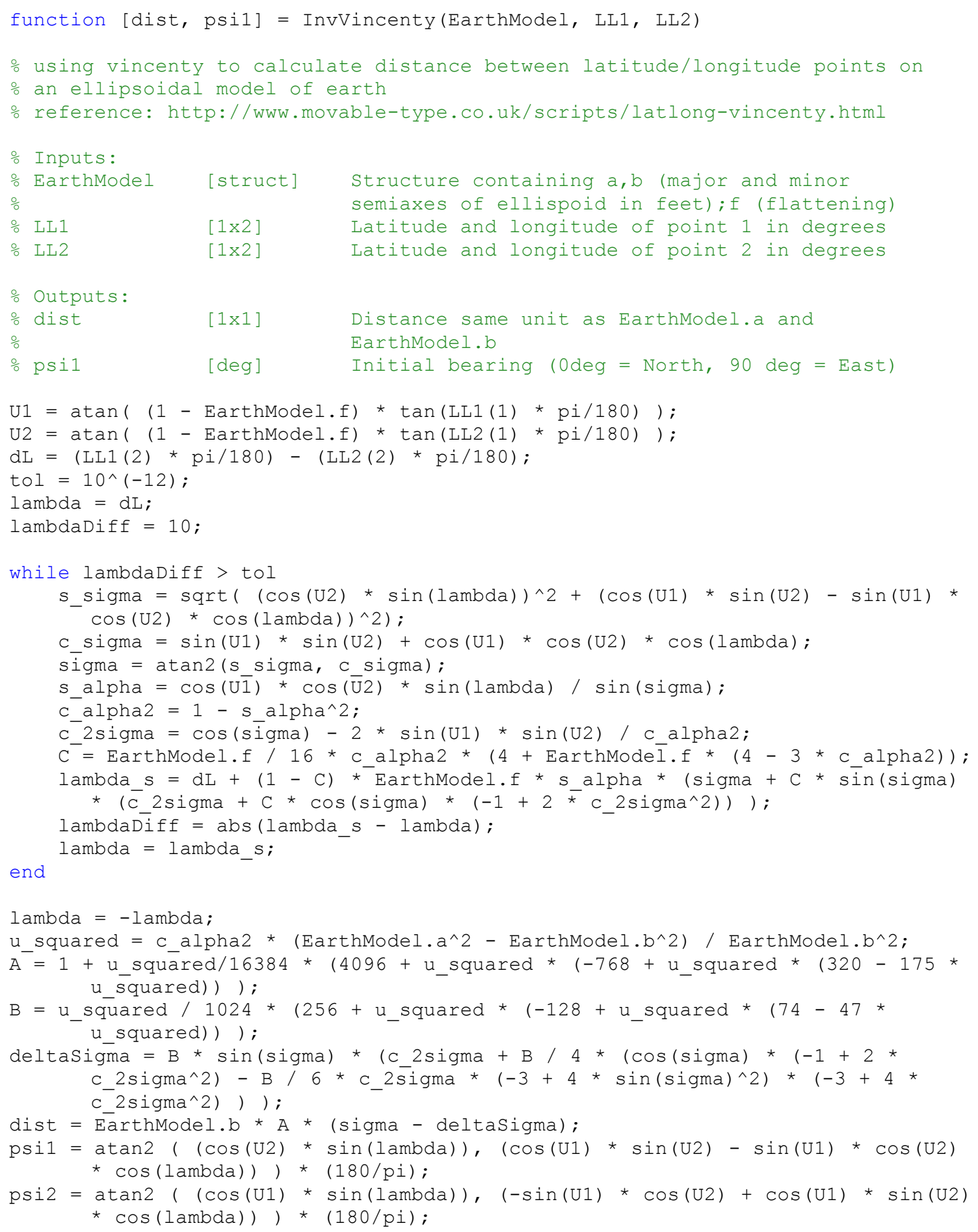




\section{Appendix C: Pseudo Control Computation for Backstepping Side slip and Bank angle Dynamics}

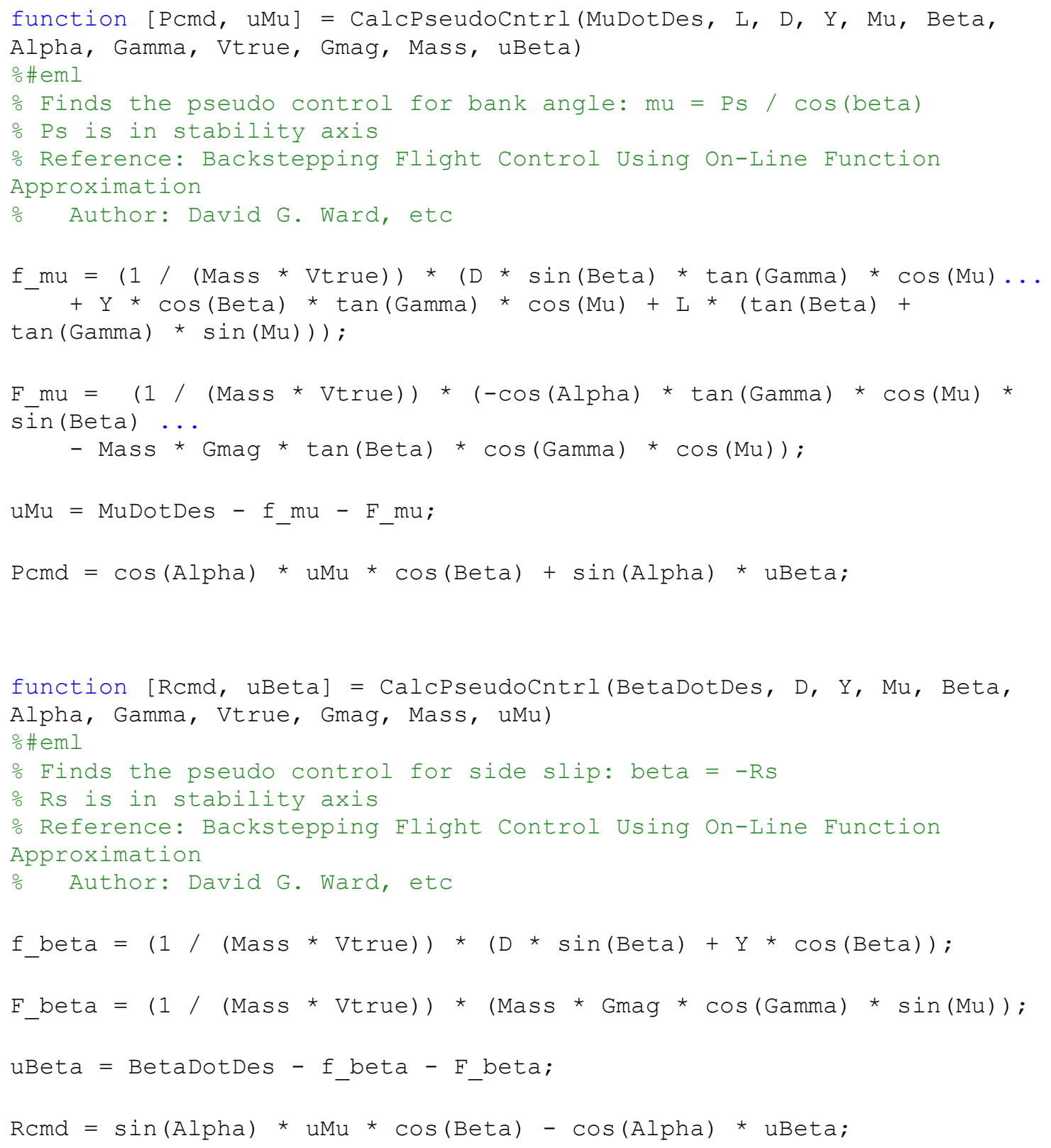

